Departamento de Inteligencia Artificial

Escuela Técnica Superior de Ingenieros Informáticos

$\mathrm{PhD}$ Thesis

\title{
Ontology verification based on lexico-syntactic patterns
}

\author{
Author: Alba Fernández Izquierdo \\ Supervisors: Dr. Raúl García Castro \\ Dr. Javier Bajo Pérez
}

November, 2020 

Tribunal nombrado por el Sr. Rector Magfco. de la Universidad Politécnica de Madrid, el día ...... de de

Presidente: Dra. Asunción Gomez Pérez

Vocal: $\quad$ Dr. Mariano Fernández Lopez

Vocal: $\quad$ Dr. Maxime Lefrançois

Vocal: $\quad$ Dr. José Manuel Molina

Secretario: Dra. Elena Montiel Ponsoda

Suplente: Dr. Sascha Ossowski

Suplente: Dra. Agnieszka Ławrynowicz

Realizado el acto de defensa y lectura de la Tesis el día ...... de de en la Escuela Técnica Superior de Ingenieros Informáticos.

Calificación:

EL PRESIDENTE

VOCAL 1

VOCAL 2

VOCAL 3

EL SECRETARIO 

A mis padres y a mi hermana.

A Roi. 



\section{Agradecimientos}

Ha llegado el día de escribir la última página de la tesis, y recordar a todas esas personas que han hecho posible que lo haya conseguido. Es difícil poner nombres, pero quiero dar las gracias a todas las personas que me han ayudado durante este camino.

En primer lugar, quiero agradecerle a Asun y a Oscar por darme la oportunidad de entrar en este grupo de investigación. Agradezco tambien a mis directores de tesis, Raúl y Javier, por haberme supervisado todos estos años. En especial, quiero darle las gracias a Raúl por su apoyo y dedicación durante esta tesis, y por todo lo que he aprendido de él en el transcurso.

Quiero agradecer también a mis compañeros de laboratorio por todas las experiencias vividas, por las charlas y por haber sido tan importantes, tanto profesionalmente como personalmente. A Chaves, Pablo, Paola, Patri, Elvi, Julia, María Navas, Ahmad, Miguel Ángel, Raúl Alcázar, Elena, Víctor, Carlos. A Andrea, por todos los consejos y por haberme aguantado todo este tiempo. A María Poveda, por ser siempre un referente. A José Ángel y Ana, por todo su tiempo.

También quiero agradecer a mis amigas que han sido necesarias para poder terminar este capítulo de mi vida. A las musampas María, Ceci, Nati, Andre, Marta A., Marta V., Tere, Sini, Sara, Lara. A Antía, por ser siempre un apoyo incondicional, aunque nos separasen miles de kilómetros. A Paula y a Mara por todos los ánimos que me habéis dado estos años. A Isa y a Lis por su alegría y fuerza para ir a por todas.

De manera muy especial quiero darle las gracias a mi familia. Sobre todo agradecerle a mis padres todo el apoyo que me han dado siempre con cariño y paciencia. Gracias por creer en mí. Sin vosotros no habría sido posible llegar hasta aquí. También quiero darle las gracias a mi hermana por haberme animado y cuidado siempre.

Finalmente, quiero darle las gracias a Roi por haber sido mi compañero todos estos años, por haberme apoyado y cuidado. Gracias por no dejarme desistir 
en los momentos más difíciles y por superar juntos todos los obstáculos que nos fuimos encontrando durante este camino. 


\begin{abstract}
Ontology verification refers to the activity where an ontology is tested against its ontology requirements, in order to ensure that it is built correctly in compliance with its ontology requirements specification. Therefore, it is a crucial activity that should be performed in any ontology development process. Since manual verification can be a time-consuming and repetitive task, testing processes to automatically verify an ontology facilitate this activity. Moreover, the involvement of not only ontology engineers during the ontology verification process, but also domain experts and users, can provide valuable feedback to avoid misunderstandings and lack of information. Therefore, this thesis proposes a method for ontology verification that defines the testing activities to be performed, as well as a testing language based on lexico-syntactic patterns to facilitate the definition of tests. To support the testing process, this thesis introduces an online tool to execute tests on one or more ontologies. In order to integrate the results of the verification activity into the development process and to allow the monitoring of such development, the thesis proposes a set of metrics extracted from the artefacts involved in the verification process, i.e., the requirements, the tests and the ontology implementation. Such metrics allow to extract information and insights related to the development of ontologies, and can be used to take managerial decisions. Finally, in order to verify whether an ontology satisfies the requirements that a standard imposes and with the aim of ensuring interoperability and quality when describing a domain, the thesis proposes a method for conformance testing grounded on the method for ontology verification. Such method analyses the overlaps and conflicts between an ontology and a standard based on the requirements of the standard.
\end{abstract}





\section{Resumen}

La verificación de ontologías es la actividad que compara una ontología con sus requisitos ontológicos, asegurando que se ha desarrollado correctamente de acuerdo con la especificación de requisitos ontológicos correspondiente. La verificación de ontologías permite asegurar que lo que se espera que esté definido en la ontología esté realmente definido en la ontología. Por ello, es una actividad crucial que debe llevarse a cabo en cualquier proceso de desarrollo ontológico. La verificación manual de ontologías puede ser una tarea tediosa y repetitiva, por lo que la ejecución automática de pruebas facilita esta actividad. Además, la colaboración tanto de desarrolladores ontológicos como de expertos de dominio y usuarios durante el proceso de verificación puede proporcionar información útil para evitar malentendidos y falta de información. Por ello, esta tesis propone un método para la verificación de ontologías. Este método incluye la definición de las actividades que deben llevarse a cabo, así como la definición de un lenguaje controlado para facilitar el diseño de las pruebas. Para dar soporte a este proceso, esta tesis presenta una herramienta online para ejecutar pruebas en una o más ontologías. Al mismo tiempo, para integrar los resultados de la verificación en el desarrollo ontológico, esta tesis propone un conjunto de métricas extraídas de los artefactos involucrados en el proceso de verificación, es decir, los requisitos, las pruebas y la implementación de la ontología. Estas métricas permiten extraer información relacionada con el desarrollo ontológico y pueden ser utilizadas para tomar decisiones a nivel de gestión. Finalmente, con el objetivo de garantizar la interoperabilidad y calidad en la descripción de un dominio, esta tesis describe un método de pruebas de conformidad, derivado del método de verificación, el cual analiza las similitudes y diferencias entre una ontología y un estándar basándose en los requisitos del estándar. 



\section{Contents}

Acronyms

1 Introduction 1

1.1 Challenges of ontology verification . . . . . . . . 2

1.2 The need for a testing process for ontology verification . . . . . . . 4

1.3 Main contributions . . . . . . . . . . . . . . . 5

1.4 Structure of the document . . . . . . . . . . . . . 7

1.5 Derived publications . . . . . . . . . . . . . . 8

1.6 Research stays . . . . . . . . . . . . . . . . . . . . . 10

1.7 Participation in research and innovation projects $\ldots \ldots \ldots$

2 State of the Art 13

2.1 Terminology ........................... 14

2.2 Related work in Software Engineering . . . . . . . . . . . . 16

2.2 .1 Software testing techniques $\ldots \ldots \ldots \ldots$

2.2.1.1 Unit testing . . . . . . . . . . . . . 16

2.2.1.2 Acceptance testing . . . . . . . . . . . 17

2.2.1.3 Keyword-driven testing . . . . . . . . . . . 17

2.2.2 Software verification testing integrated into software development methodologies . . . . . . . . . . . . . . . . . . 18

2.2.2.1 Test-driven development . . . . . . . . . . . . . . . 18

2.2.2.2 Behaviour-driven development . . . . . . . . . . . 18

2.2.3 Software testing conformance methods . . . . . . . . . . . . 20

$2.2 .3 .1 \quad \mathrm{ISO} / \mathrm{IEC} 9646 \ldots \ldots \ldots \ldots$

$2.2 .3 .2 \quad$ ETSI . . . . . . . . . . . . . . . . . 21 
$2.2 .3 .3 \mathrm{~W} 3 \mathrm{C} \ldots \ldots \ldots \ldots \ldots$

2.2.4 Software metrics to support the software life-cycle . . . . . . 23

2.2.4.1 Source code product metrics . . . . . . . . . . 24

2.2.4.2 Requirements specification product metrics . . . . . 25

2.2.4.3 Test case product metrics . . . . . . . . . . . 27

2.2.4.4 Software documentation product metrics . . . . . 27

2.2.4.5 Process metrics . . . . . . . . . . . . . . . . 27

2.3 Related work in Ontology Engineering . . . . . . . . . . . . 28

2.3.1 Requirements specification in ontology development methodologies . . . . . . . . . . . . . . . . . . . . . 28

2.3.1.1 Grüninger and Fox . . . . . . . . . . . . . 30 30

2.3.1.2 ON-TO-KNOWLEDGE . . . . . . . . . . . . 31

2.3.1.3 EXtreme Ontology . . . . . . . . . . . . . . 32

2.3.2 Tests definition in ontology development techniques . . . . . . . 33

2.3.2.1 eXtreme Design _ . . . . . . . . . . . . . . . . . 33

2.3 .2 .2 SAMOD . . . . . . . . . . . . . . 35

2.3.3 Requirements analysis for ontology verification testing . . . . . 36

2.3.3.1 Ren and colleagues' requirement analysis . . . . . . . 36

2.3.3.2 Wiśniewski and colleagues' requirement analysis . . . . 38

2.3.4 Ontology verification testing methods . . . . . . . . . . . 40

2.3.4.1 Blomqvist and colleagues' testing approach . . . . . . 40

2.3.4.2 CQChecker ..................... 41

2.3.5 Ontology testing methods for query execution . . . . . . . 42

2.3.5.1 OntologyTest . . . . . . . . . . . . . . . . . 43

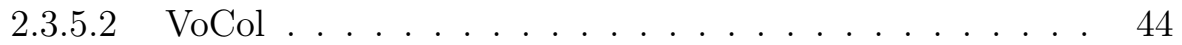

2.3.6 Ontology testing integrated into ontology development methodologies . . . . . . . . . . . . . . . . . . . . 4 45

2.3.7 Ontology metrics to support the ontology life-cycle . . . . . . . 47

2.3.7.1 Metrics related to ontology classes . . . . . . . . . . 47

2.3.7.2 Metrics related to the class hierarchy . . . . . . . . 4 49

2.3.7.3 Metrics related to ontology properties . . . . . . . . 50

2.3.7.4 Metrics related to ontology individuals . . . . . . . . 50 50

2.3.7.5 Metrics related to ontology axioms . . . . . . . . . . 50 
2.3.7.6 Metrics related to ontology metadata . . . . . . . 51

2.4 Conclusions . . . . . . . . . . . . . . . . . . . . 51

3 Work Objectives

3.1 Thesis objectives . . . . . . . . . . . . . . . 5 57

3.2 Contributions . . . . . . . . . . . . . . . . . 59

3.3 Work assumptions, hypotheses and restrictions . . . . . . . . . . 61

3.4 Evaluation $\operatorname{plan} \ldots \ldots \ldots \ldots \ldots$. . . . . . . . . . . . . . . . . .

3.5 Research methodology . . . . . . . . . . . . . . . . 65

3.5.1 Description of the research process . . . . . . . . . 68 68

4 Method for ontology verification testing 71

4.1 Ontology requirements specification analysis . . . . . . . . . . . 71

4.1 .1 Building the CORAL corpus . . . . . . . . . . 72

4.1.2 Dictionary of lexico-syntactic patterns . . . . . . . . 76

4.1.3 Annotated corpus of ontology requirements . . . . . . . . . . 79

4.2 Activities within the ontology verification process . . . . . . . . . 79

4.2 .1 Test design activity . . . . . . . . . . . . . . . . 81

4.2 .2 Test implementation activity . . . . . . . . . . 84

4.2 .3 Test execution activity . . . . . . . . . . . . . . 86

4.3 Verification Test Case ontology . . . . . . . . . . . . . . . . 88

4.4 Technological support for ontology testing: Themis . . . . . . . . . . . 91

4.4.1 System architecture . . . . . . . . . . . . . . . 91

4.4 .2 Themis REST API . . . . . . . . . . . . . . . . . . . . . 93

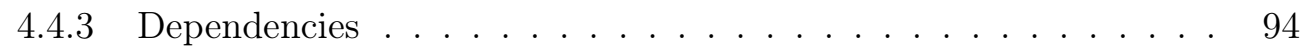

5 Ontology metrics derived from the ontology verification process 95

5.1 Ontology metrics identification . . . . . . . . . . . . . . . . 96

5.2 Roles in Ontology Engineering . . . . . . . . . . . . . . . . . . . 97

5.3 Development artefacts in the ontology verification process . . . . . . 9 99

5.3.1 Ontology requirements specification document . . . . . . . . . . 99

5.3 .2 Ontology requirements test suite . . . . . . . . . . . . . 100

5.3 .3 Ontology implementation . . . . . . . . . . . . . . . 100

5.4 Ontology Engineering metrics . . . . . . . . . . . . . . . . 100 
5.4 .1 Base product metrics . . . . . . . . . . . . . . . . 101

5.4 .2 Calculated product metrics . . . . . . . . . . . . 104

5.4 .3 Process metrics . . . . . . . . . . . . . . . . . . 105

6 Ontology conformance testing 107

6.1 Method for ontology conformance testing . . . . . . . . . . . . . 108

6.1.1 Activities within the method for conformance testing . . . . . . 108

6.1.1.1 Functional ontology requirements extraction . . . . . 109

6.1.1.2 Test design . . . . . . . . . . . . . . 110

6.1.1.3 Test implementation . . . . . . . . . . . . . . . 111

6.1.1.4 Test execution . . . . . . . . . . . . 112

6.1 .1 .5 Test results analysis $\ldots \ldots \ldots \ldots . . \ldots \ldots 112$

6.1.2 Technological support: Themis . . . . . . . . . . . . . 115

6.2 Method for minimum common knowledge identification . . . . . . . . . 116

6.2.1 Activities within the minimum common knowledge identification

$\operatorname{method} \ldots \ldots \ldots \ldots \ldots \ldots$

6.2.1.1 Generation of ontologies from data models . . . . . . . 117

6.2.1.2 Generation of a shared glossary of terms . . . . . . . 118

6.2.1.3 Analysis of the results . . . . . . . . . . . . . . . 119

6.2 .2 Technological support: Themis . . . . . . . . . . . . . 119

7 Evaluation $\quad \mathbf{1 2 1}$

7.1 Evaluation of the method for ontology verification testing . . . . . . 121

7.1 .1 Experimental design . . . . . . . . . . . . . . . . 122

7.1 .2 Results and discussion . . . . . . . . . . . . . . . . 125

7.1 .2 .1 Time analysis . . . . . . . . . . . . . 126

7.1.2.2 Correctness analysis . . . . . . . . . . . . . 127

7.1.2.3 Usefulness, satisfaction and ease of use analysis . . . . 136

7.1.3 Conclusions of the experiment . . . . . . . . . . . . . . 139

7.2 Evaluation of the metrics derived from the verification process . . . . . 139

7.2 .1 Experimental design . . . . . . . . . . . . . . . . . 140

7.2 .2 Results and discussion . . . . . . . . . . . . . . . . . . . . 142

7.2.2.1 Influence derived from the number of requirements . . . 142

7.2.2.2 Influences derived from requirements complexity . . . . 147 
7.2.2.3 Influences derived from the volatility of requirements . . 151

7.2.2.4 Influences derived from the number of tests . . . . . 153

7.2.2.5 Influences derived from the number of tested terms . . 155

7.2.3 Conclusions of the experiment . . . . . . . . . . . . 155

7.3 Evaluation of the method for ontology conformance testing . . . . . . 156

7.3.1 Conformance testing method . . . . . . . . . . . . 157

7.3.1.1 Experiment design . . . . . . . . . . . . . . . 157

7.3.1.2 Results and discussion . . . . . . . . . . . . 158

7.3.2 Minimum common knowledge between ontologies . . . . . . . . 165

7.3.2.1 Experiment design . . . . . . . . . . . . . 165

7.3.2.2 Results and discussion . . . . . . . . . . . . 166

7.3.3 Conclusions of the experiments . . . . . . . . . . . . . 170

8 Conclusions and future work

A Test implementation catalogue 193

B Axioms associated to test expressions 211

C Usefulness, satisfaction, and ease of use questionnaire about tools for ontology verification 



\section{List of Figures}

2.1 Test-driven development steps (Bhat and Nagappan, 2006) . . . . . . 19

2.2 Summary of ISO/IEC 9646 activities for conformance testing . . . . . . 22

2.3 Development of ETSI test specifications (Moseley et al. 2003) . . . . . . 22

2.4 Summary of the development and testing methods in Ontology Engineering 29

2.5 Procedure for ontology design and evaluation (Grüninger and Fox, 1995) 30

2.6 On-To-Knowledge development phases (Staab et al., 2001) . . . . . . . 31

2.7 The overall XD process $($ Presutti et al. 2009$) \ldots \ldots$. . . . . . . . 34

2.8 A brief summary of SAMOD $($ Peroni, 2016) $\ldots \ldots \ldots \ldots$

2.9 Main concepts of the TestCase metamodel (Blomqvist et al., 2012) . . . 41

2.10 Architecture of CQChecker (Bezerra et al. 2014) . . . . . . . . . 4 42

$2.11 \mathrm{VoCol}$ architecture and workflow $($ Halilaj et al. 2016) . . . . . . . . 44

2.12 Summary of the possible ontology lifecycle that focuses on TDD, and the sequence of steps of the TDD procedure (Keet and Ławrynowicz, 2016) . 46

3.1 Correspondences between objectives, contributions, hypotheses, restrictions and assumptions . . . . . . . . . . . . . . 64

3.2 Inputs and context considered for creating the testing framework . . . 66 66

3.3 Phases of the thesis development, including the tasks carried out during each phase and the main publications derived from each phase. . . . . . 69

4.1 Steps, with their inputs and outputs, carried out to conduct the analysis and to create CORAL. . . . . . . . . . . . . . . . . . .

4.2 Testing activities during the testing process, together with their inputs

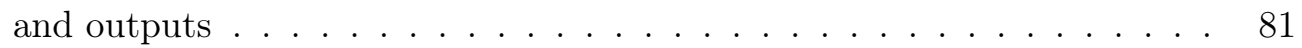

4.3 Overview of the Test Case Verification ontology $\ldots \ldots \ldots$. . . . . . 88 
4.4 Themis architecture overview $\ldots \ldots \ldots \ldots$. . . . . . . . . . . 92

5.1 Activity workflow followed to generate the proposed set of metrics . . . . 97

5.2 Ontology engineering metrics and interested actors. . . . . . . . . . . 102

6.1 Ontology conformance testing approach in ontology engineering . . . . . 109

6.2 Conformance approach for identifying the minimum commitment between ontologies . . . . . . . . . . . . . . . . . 117

7.1 Comparison of the time spent per requirement for participants not familiar with the OWL language . . . . . . . . . . . . . . . 126

7.2 Comparison of the time spent per requirement for participants familiar with the OWL language . . . . . . . . . . . . . . . . . . 127

7.3 Comparison of the time spent per requirement for participants experts with the OWL language . . . . . . . . . . . . . . . . . . . . . . . 127

7.4 Summary of the usefulness results . . . . . . . . . . . . . . 136

7.5 Summary of the satisfaction results . . . . . . . . . . . . 136

7.6 Summary of the usability results . . . . . . . . . . . . . . 137

7.6 Summary of the usability results (Cont.) . . . . . . . . . . . . . 138

7.7 Aspects to be analysed and their associated metrics . . . . . . . . . . . . 141

7.8 Scatterplot related to NReqs and NTests . . . . . . . . . . . . . 144

7.9 Size of the ontologies during their development process not including imported ontologies . . . . . . . . . . . . . . . . . . . . 144

7.10 Size of the ontologies during their development process including imported ontologies . . . . . . . . . . . . . . . . . . 145

7.11 Scatterplot related to NReqs and NA (without imports) . . . . . . . 146

7.12 Requirement development average time for each ontology version . . . . 146

7.13 Distribution of the requirements axiom complexity in each ontology in the last sprint . . . . . . . . . . . . . . . . . . . . . . . . . . . 1499

7.14 Distribution of the volatility of the requirements in each ontology in the last sprint . . . . . . . . . . . . . . . . . . 152

7.15 Scatterplot related to the requirements volatility (ReqVolatility) and the

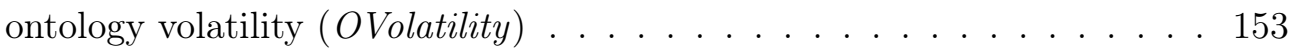

7.16 Distribution of the test and requirement coverage in each ontology version 154 
7.17 Influences between metrics obtained during the experiment . . . . . . 156

7.18 Shared properties and classes between IoT standards and the VICINITY ontology network . . . . . . . . . . . . . . . . . . 164

7.19 Overview of the shared terms between standards $\ldots \ldots \ldots$. . . . . 170 



\section{List of Tables}

2.1 Competency question archetypes $($ Ren et al. 2014 $\ldots . . . . . . .38$

2.2 Summary of conclusions regarding ontology testing within ontology development methodologies . . . . . . . . . . . . . . . . 53

2.3 Summary of conclusions regarding ontology testing approaches . . . . 55

2.4 Main characteristics of the conformance testing approaches . . . . . . 554

2.5 Summary of metrics in Ontology Engineering . . . . . . . . . . . . . 55

4.1 Collected ontologies together with their requirements that have been analysed to create CORAL . . . . . . . . . . . . . . 74

4.2 List of lexico-syntactic patterns included in the dictionary . . . . . . 77

4.3 OWL constructs and DL expressivity associated with each lexico-syntactic

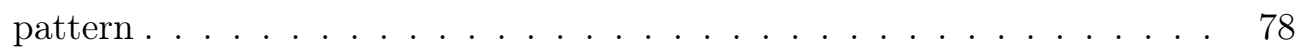

4.4 Number of requirements annotated with each lexico-syntactic pattern . . 80

4.5 List of proposed test expressions together with their category . . . . . . 83

4.6 T4. Equivalence between two classes A and B . . . . . . . . . . . 8 85

5.1 Base product metrics and artefacts from which they are extracted . . . . 103

5.2 Calculated product metrics and artefacts from which they are extracted 104

5.3 Process metrics . . . . . . . . . . . . . . . . . . . 106

6.1 Implementation of a test expression for checking subsumption between the classes Gateway and DigitalEntity . . . . . . . . . . . . . . 111

6.2 Example of association between tests and axioms . . . . . . . . . . 118

6.3 Example of a shared glossary of terms . . . . . . . . . . . . . 118

7.1 List of requirements . . . . . . . . . . . . . . . 122 
7.2 OWL expertise of participants in the experiment . . . . . . . 125

7.3 Development expertise of participants in the experiment . . . . . . . 125

7.4 Percentage of correctness results for Themis . . . . . . . . . . . . . 128

7.5 Percentage of correctness results for TDDOnto $2 \ldots \ldots$. . . . . . 129

7.6 Percentage of correctness results for Protégé . . . . . . . . . . . 130

7.7 Summary of the error results . . . . . . . . . . . . . . . 131

7.8 Percentage of results for Themis grouped by type of requirement . . . . 132

7.9 Percentage of results for TDDOnto2 grouped by type of requirement . . 133

7.10 Percentage of results for Protégé grouped by type of requirement . . . . 134

7.11 Summary of the results per tool . . . . . . . . . . . . . . . . 135

7.12 Number of requirements and tests in each sprint ( $\mathrm{R}$ refers to Requirements and $\mathrm{T}$ refers to Tests $\ldots \ldots$. . . . . . . . . . . . 143

7.13 DL expressivity of the ontologies during each sprint . . . . . . . . . . 147

7.14 Volatility of the ontologies (OVolatility) during each sprint . . . . . . . 151

7.15 Summary of requirements information for the IoT standards . . . . . . . 160

7.16 Summary of testing results of the IoT standards for the VICINITY ontology network . . . . . . . . . . . . . . . . . 160

7.17 Summary of the types of tests passed by the VICINITY ontology . . . . 161

7.18 Summary of the topics of each standard and the Passed Test Percentage of the VICINITY ontology network . . . . . . . . . . . . . . 162

7.19 Shared requirements between the IoT standards . . . . . . . . . . . 167

7.20 Classes shared by the analysed standards . . . . . . . . . . . . . 169

7.21 Properties shared by the analysed standards . . . . . . . . . . . . . . . 170

A.1 T1. Class A exists . . . . . . . . . . . . . . . . . . . 193

A.2 T2. Subsumption relation between classes A and B . . . . . . . . . . 193

A.3 T3. Disjointness between two classes A and B . . . . . . . . . . . . . . 194

A.4 T4. Equivalence between two classes A and B . . . . . . . . . . . . . . 194

A.5 T5. Property P exists . . . . . . . . . . . . . . . . 194

A.6 T6. Existential relation P between two classes A and B . . . . . . . . 195

A.7 T7. Universal relation P between two classes A and B . . . . . . . . . 195

A.8 T8. Symmetric property P . . . . . . . . . . . . . . . . . 196

A.9 T9. Minimum cardinality . . . . . . . . . . . . . . 196 
A.10 T10. Maximum cardinality . . . . . . . . . . . . . 196

A.11 T11. Exact cardinality . . . . . . . . . . . . . . . . . . 197

A.12 T12. Universal relation P between the union of two classes A and B . . 197

A.13 T13. Universal relation $\mathrm{P}$ between the intersection of two classes A and B 198

A.14 T14. Individual I exists . . . . . . . . . . . . . . . . 198

A.15 T15. Property P has domain Class A . . . . . . . . . . . . . . . 198

A.16 T16. Property P has range Class A . . . . . . . . . . . . . . . . . . 199

A.17 T17. Multiple inheritance of a class . . . . . . . . . . . . . . . . 199

A.18 T18. There is a subsumption relation between two classes and an exis-

tential restriction . . . . . . . . . . . . . . . . . 200

A.19 T19. Minimum cardinality and existential relation . . . . . . . . . 201

A.20 T20. Minimum cardinality and universal restriction . . . . . . . . . 202

A.21 T21. Subsumption relation between A and B, subsumption relation be-

tween $\mathrm{A}$ and $\mathrm{C}$, and disjointness between $\mathrm{B}$ and $\mathrm{C} \ldots \ldots . . . \ldots 203$

A.22 T22. Participation ODP between classes A and B . . . . . . . . . . . 204

A.23 T23. Co-participation ODP . . . . . . . . . . . . . . . . 205

A.24 T24. PartOf ODP between classes A and B . . . . . . . . . . . 206

A.25 T25. PartOf ODP between classes A and B with universal restriction . 207

A.26 T26. Object-Role ODP between classes A and B with existential restriction208

A.27 T27. Object-Role ODP between classes A and B with universal restric-

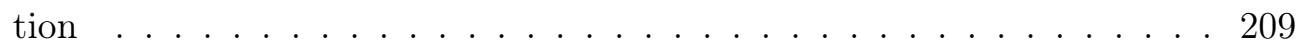

A.28 T28. Two individuals of classes A and B can be related by property P . 209

B.1 Axioms associated to test expressions . . . . . . . . . . . . . . . . 211 



\section{List of Algorithms}

1 Test execution ....................... 87 



\section{Acronyms}

API: Application Programming Interface

ATS: Abstract Test Suite

CQ: Competency Question

BDD: Behaviour-Driven Development

ETS: Executable Test Suite

GUI: Graphical User Interface

ICS: Implementation Conformance Statement

IXIT: Implementation Extra Information for Test

ORSD: Ontology Requirement Specification Document

ORT Ontology Requirement Test Suite

OWL: Web Ontology Language

RDF: Resource Description Framework

SPARQL: SPARQL Protocol and RDF Query Language

TDD: Test-Driven Development

TSS: Test Suite Structure

TTCS: Testing and Test Control Notation

TP: Test Purpose

URI: Uniform Resource Identifier

URL: Uniform Resource Locator 



\section{Chapter 1}

\section{Introduction}

Ontology Engineering can be understood as the discipline that investigates the principles, methods and tools for initiating, developing and maintaining ontologies (GómezPérez et al., 2004). This discipline provides life cycles that go from requirements definition to the maintenance of ontologies, as well as methodologies, techniques and tools to support and drive the development of ontologies.

During their development, ontologies need proper quality control to be effectively deployed in practical applications (Guarino, 2004), preventing inconsistencies, mistakes and redundancies. Several ontology development methodologies (for example, METHONTOLOGY (Fernández-López et al., 1997) or NeOn (Suárez-Figueroa et al. 2015)) propose an evaluation activity within every ontology development project, which benefits the quality of the ontology being built.

The Ontology Engineering discipline has had an active research on ontology evaluation. In 1995, Gomez-Pérez and colleagues (Gómez-Pérez et al., 1995) contributed with the definition of ontology evaluation, while Grüninger and Fox (Grüninger and Fox, 1995) introduced an evaluation method against a set of requirements called competency questions. Such competency questions refer to a set of questions written in natural language that the ontology must be capable to answer. The interest in ontology evaluation has been growing since then and several works have emerged, focused on different aspects of ontology evaluation, mostly related to the ontology model. For example, there are approaches that are oriented to the assessment of errors in ontology modelling Guarino and Welty, 2009; Poveda-Villalón et al., 2014), to the assessment of the quality of ontologies by using metrics based on the ontology model (Duque-Ramos et al. 
2011; Gangemi et al., 2006: Lozano-Tello and Gómez-Pérez, 2004) or to pattern-based evaluation (Djedidi and Aufaure, 2010; Presutti et al., 2008).

Following the proposal for the ontology evaluation activity presented by SuarezFigueroa and colleagues in NeOn (Suárez-Figueroa et al., 2015), ontology evaluation is divided into validation and verification. On the one hand, during the validation activity the meaning of the ontology definitions is compared against the intended model of the world aiming to conceptualise. On the other hand, during the verification activity the ontology is compared against its ontology requirements, ensuring that the ontology is built correctly in compliance with the ontology requirements specification (SuárezFigueroa et al., 2013).

Ontology Engineering has, over the years, been inspired by Software Engineering practices. Regarding ontology verification, there is still much to learn from the software verification body of knowledge. In Software Engineering, software verification practices and techniques are widely integrated into the software development process to assure the quality of software products. Moreover, to be aware of the status of the software development process and to be able to plan and manage it efficiently, a diversity of metrics related to artefacts generated and used during the verification process have been defined. Such metrics enable software engineers to have enough information to make different types of predictions, assessments and trade-offs, such as effort and time predictions or software quality analysis (Fenton and Neil, 2000).

Moreover, the result of the verification process and the metrics can also be used to determine to what extent a software product conforms to the requirements of a standard (Moseley et al., 2003), being able to analyse software conformance for ensuring that a software product is reliable and interoperable (Graydon et al. 2012). The adoption of these practices and techniques in Software Engineering can be beneficial for the Ontology Engineering field.

\subsection{Challenges of ontology verification}

Ontology verification is a well-known activity that should be carried out within any ontology development process, and it is mentioned in many ontology development methodologies (Peroni, 2016; Staab et al., 2001). Since manual verification can be a time- 
consuming and repetitive task, latest works propose the use of tests to automatise and facilitate the verification of ontologies (Peroni, 2016).

The creation of such tests is not a straightforward task and latest work has focused on the analysis of patterns in sets of requirements written as competency questions, with the ultimate goal of supporting the automatic formalisation of such requirements into SPARQL queries (Ren et al., 2014, Wiśniewski et al., 2019). However, one of the main issues related to the ontology verification activity is the ambiguity of ontology requirements, which hinders their formalisation into tests and their translation into axioms.

There are some testing approaches that can be applied to ontology verification, such as the work of Keet and Ławrynowicz (Keet and Ławrynowicz, 2016), which proposes a Test-driven development of ontologies, or the work of Blomqvist and colleagues (Blomqvist et al., 2012), which proposes several types of tests (e.g., inference verification or error provocation) to be executed on an ontology. However, these works do not cover the workflow from ontology requirements into tests and are only focused on the definition of different types of tests in order to check a given criteria. Therefore, the step of how to generate those tests given a set of requirements is out of scope of these existing works.

Furthermore, the majority of the verification approaches in the state of the art are oriented to provide tests with the aim of helping ontology engineers to know whether the requirements defined for a particular ontology are satisfied by it. Consequently, they do not consider any input from users or domain experts, which are usually excluded from this verification activity. However, the involvement of domain experts and users during the ontology verification activity could be beneficial for developers. As an example, in Software Engineering the involvement of users in the development process is claimed to have a positive impact on the success of the software (Begier, 2010; Kujala, 2003).

Inspired by the Software Engineering field and its verification techniques, the information obtained during the ontology verification process should be monitored during the ontology development process. In real-world projects it is necessary to be aware of the status of the product, i.e., the ontology, at every step of the development process, in order to be able to plan and manage its development in an efficient way. Currently, different metrics exist which try to assess the quality of ontologies by measuring reliability, reusability or cohesion, among other aspects. However, the metrics proposed 
until now are mostly focused on the ontology implementation and they do not take into account other artefacts produced during the ontology development process or even the development process itself.

Moreover, several standardisation bodies, such as the European Telecommunications Standards Institute (ETSI) or the World Wide Web Consortium (W3C), are currently developing standard ontologies ${ }^{1}$ in order to maximise semantic interoperability between models. All these standard ontologies are created in order to provide an agreed baseline approved by the community that can be used by other ontology engineers who want to describe the related area of concern. Therefore, it should be possible to ensure that the ontologies of a particular domain verify the requirements of the related standards, i.e., conform to them, ensuring quality and interoperability when describing the domain. Moreover, in this scenario it is important that the tests are defined independently of any implementation, in order to be executed on other ontologies to check whether they meet the requirements of the standard.

\subsection{The need for a testing process for ontology verification}

The design of tests is not a trivial task, especially if tests are written in a formal language such as SPARQL. This is one of the reasons of the rise of testing syntaxes in the Software Engineering field, such as Gherkin ${ }^{2}$ which emerged with the aim of describing testing behaviours without the need to go into implementation details. With them, tests can be easily understood by experts and non-experts, and they can be automatically executed on a software product.

In Ontology Engineering, it should also be considered that, in addition to ontology engineers, domain experts and users can also be involved in the ontology testing activity. The feedback between them and ontology engineers can help identify misunderstandings in requirements or lack of information at an early stage of the development process. Moreover, involving users would increase their confidence in the correctness of an ontology, since they will be able to check and understand the testing results. Therefore, a verification testing process should define a particular testing language that facilitates

\footnotetext{
${ }^{1}$ In this thesis standard ontologies refers to those developed by standardisation bodies. 2https://cucumber.io/docs/gherkin/
} 
the definition of tests from requirements, allowing developers, domain experts and users to be involved in the testing process.

Ontology tests and requirements can be understood as a formal documentation of an ontology. Furthermore, the traceability between tests, requirements and ontology implementation allows ontology engineers to have an overview of whether the ontology is being built according to the specified requirements, and also of whether all requirements are included into the tests. Moreover, it facilitates the identification of missing knowledge defined in the ontology.

The definition of specific metrics based on tests, their associated requirements and the ontology implementation, allows having more control over the ontology development process. Moreover, these metrics can provide the needed information to lead to evidencebased decision-making and to an improvement in the efficiency of the development process.

Specifying ontology requirements and their associated tests during ontology development activities opens the door to other related use cases. For example, such requirements and tests can be used for analysing the conformance between an ontology and a standard to determine whether the ontology meets the requirements of the standard, or whether there are conflicts between them. Furthermore, both requirements and tests can be used to identify common knowledge or the presence of conflicts between a set of standards related to the same domain, which is valuable information for identifying potential mappings and needs that are not supported by existing standards or that are defined in more than one.

\subsection{Main contributions}

The state of the art in ontology verification, as well as the need for a testing process for ontology verification, has driven the definition of the following objectives and contributions in this thesis:

1. The first objective is to help ontology engineers, domain experts and users to test whether their ontologies verify the expected ontology requirements. To achieve this goal, the following contributions are developed in this thesis: 
- An ontology testing method for ontology verification based on ontology requirements. This method analyses how ontology requirements are specified and generates a testing language based on them in order to facilitate the verification process. Moreover, it defines a set of activities to be carried out in order to systematise the verification process. These activities are divided into test design, test implementation and test execution, separating the design of the test from its implementation and, therefore, increasing its maintenance. Moreover, the results of the verification process are stored using RDF format, allowing to specify the traceability between requirements, tests and the ontology implementation.

- Themis, an online tool for ontology testing. This online tool allows to execute tests on one or multiple ontologies using the proposed testing language and following the activities proposed in the testing method.

2. The second objective is to extract information that supports making decisions during ontology development by means of metrics. To achieve this goal, the following contribution is developed in this thesis:

- A set of metrics related to the artefacts generated during the ontology verification process. These metrics combine information extracted from the ontology implementation, the requirements and the tests in order to provide information about the status of the ontology verification in particular and the ontology development process in general.

3. The third objective is to automate the assessment of conformance between ontologies and standards to check up to what extent ontologies verify the requirements of standards. To achieve this goal, the following contribution is developed in this thesis:

- An ontology testing method for ontology conformance. This method is focused on determining the degree of conformance between an ontology and a standard by using tests, as well as by computing metrics related to the requirements, their tests and the ontology. The method defines abstract tests (i.e., tests that are independent of any ontology implementation); therefore, these tests can be executed in any ontology, although they are extracted 
from the standard. Based on the ontology conformance testing method, a minimum common knowledge identification method is also proposed, which allows analysing the conformance between a set of standards. For those standards that define a data model but not an ontology, this method generates an ontology from a set of tests. Consequently, it supports the analysis not only between standard ontologies, but also between other non-ontological standard data models.

This thesis is focused on the verification activity regarding the functional requirements of ontologies, i.e., requirements that refer to the particular knowledge to be represented by the ontology (Suárez-Figueroa et al., 2009b). Therefore, for the sake of readability, along this document the term "requirement" will refer to functional requirement.

\subsection{Structure of the document}

The thesis is structured as follows:

- Chapter 2 provides a general vision of the existing work in the areas related to this thesis. This chapter is divided into two parts. The first one frames the testing methods, metrics and conformance testing approaches proposed in the Software Engineering field. Afterwards, relevant testing methodologies, tools and metrics in Ontology Engineering are described. Finally, some conclusions are given.

- Chapter 3 defines the objectives, contributions, assumptions, hypotheses and restrictions for this thesis. This chapter also includes the research methodology followed and the evaluation plan of the hypotheses.

- Chapter 4 presents the ontology verification testing method for systematising the definition, implementation and execution of tests extracted from requirements. The chapter describes the analysis of requirement specifications, the description of ontology testing activities based on such requirement specifications, the proposed ontology to store tests and the technological support. 
- Chapter 5 describes the set of ontology metrics extracted from different artefacts generated and used during the ontology verification process, such as the ontology implementation, the requirements or the tests.

- Chapter 6 describes the conformance scenarios where the ontology verification testing method could be adopted, including ontology conformance testing and minimum common knowledge identification.

- Chapter 7 presents the evaluation of the main contributions of the thesis.

- Chapter 8 draws some conclusions and presents ideas for future work.

- Annex A lists the test implementations for all the tests supported by the testing language defined in Chapter 4.

- Annex B lists all the associations between tests and ontology axioms that are used in Chapter 6 .

- Annex C presents the USE questionnaire given to the users in order to evaluate the contributions in Chapter 4.

\subsection{Derived publications}

During the development of this thesis, different results were accepted in journals and published in international conferences with peer review. Such publications, and other outcomes, are gathered in this section.

\section{- Journal publications}

- Fernández-Izquierdo, A., and García-Castro, R.. Conformance testing of ontologies through ontology requirements. Engineering Applications of Artificial Intelligence. This work presents the ontology conformance testing method described in Chapter 6 .

- Fernández-Izquierdo, A., Poveda-Villalón, M., Gómez-Pérez, A. and GarcíaCastro, R.. Towards metrics-driven ontology engineering. Knowledge and Information Systems. This work presents the set of ontology metrics described in Chapter 5 . 


\section{- Conference publications}

- Fernández-Izquierdo, A., and García-Castro, R.. Requirements Behaviour Analysis for Ontology Testing. 21st International Conference on Knowledge Engineering and Knowledge Management, EKAW 2018. Nancy, France 12nd - 16th November, 2018. This work presents the ontology verification testing method described in Chapter 4 .

- Fernández-Izquierdo, A., Poveda-Villalón, M., and García-Castro, R. CORAL: A Corpus of Ontology Requirements Annotated with Lexico-Syntactic Patterns. 16th Extended Semantic Web Conference, ESWC 2019. Portoroz, Slovenia 2nd - 6th June, 2019. This work presents the corpus of requirements described in Chapter 4

- Fernández-Izquierdo, A., and García-Castro, R. Themis: a tool for validating ontologies through requirements. 31st International Conference on Software Engineering and Knowledge Engineering, SEKE 2019. Lisbon, Portugal 10th - 12nd July, 2019. This work presents the technological support described in Chapter 4.

\section{- PhD symposium}

- Fernández-Izquierdo, A. Ontology testing based on requirements formalisation in collaborative development environments. Doctoral Consortium at the 16th International Semantic Web Conference, ISWC 2019. Vienna, Austria 21st - 25th October, 2017. This publication presented the first approach towards the testing method described in Chapter 4

\section{- Demo}

- Fernández-Izquierdo, A. and García-Castro, R. How to validate ontologies with Themis. Demo session at the 16th Extended Semantic Web Conference, ESWC 2019. Portoroz, Slovenia 2nd - 6th June, 2019.

\section{- Tutorials}


- Fernández-Izquierdo, A., Alobaid, A. and Poveda-Villalón, M. Catching up with ontological engineering - To git-commit and beyond. 21st International Conference on Knowledge Engineering and Knowledge Management, EKAW 2018. Nancy, France 12nd - 16th November, 2018. In this tutorial the ontology verification testing approach was introduced as an activity integrated into the ontology development process.

- Poveda-Villalón, M. and Fernández-Izquierdo, A. Integrating ontological development with software engineering trends. Forum on specification and Design Languages, FDL 2019. Southampton, United Kingdom 2nd - 4th September, 2019. In this tutorial the tool Themis, which is described in Chapter 4, was presented.

\subsection{Research stays}

During the development of this thesis, research stays took place in the following research institutions:

- Wright State University at Ohio, USA, from August 28th to September 28th 2017 supervised by Dr. Pascal Hitzler within the DaSe Lab for Data Semantics. During this stay the author analysed different methods and tools to verify ontologies.

- Insight Centre for Data Analytics at Galway, Ireland, from September 9th to December 11th 2019 supervised by Dr. Edward Curry. During this stay, the author worked on the evaluation of this thesis, collaborating with researchers and applying her work into an H2020 project where the Insight Center is involved. This stay was funded by the Convocatoria de Ayudas al personal investigador en formación predoctoral para realizar una estancia de investigación internacional igual o superior a tres meses de la Universidad Politécnica de Madrid program.

\subsection{Participation in research and innovation projects}

During the development of this thesis, the author has acquired practical experience in Ontology Engineering through the participation in the following research and innovation projects: 
- VICINITY project (H2020-688467) [September 2016 - July 2019]: during the author's collaboration in this European Project she acquired knowledge about methodologies and tools for building and evaluating ontologies. This project was also a real use case for experimentation with the technological support proposed in this thesis.

- ETSI STF 534 [May 2018 - April 2019]: this international project funded by the European Telecommunications Standards Institute (ETSI) provided the author with experience in ontology development in a distributed environment and in conformance testing.

- DELTA project (H2020-773960) [May 2019 - November 2020]: in this European project the author gained experience in the ontology development as well as in the specification and verification of requirements with domain experts. 



\section{Chapter 2}

\section{State of the Art}

This chapter presents the state of the art of the topics of interest related to this thesis, with the purpose of providing an overview of the covered fields and of identifying open research problems. First of all, significant definitions about ontology verification, ontology testing and related terms are provided in Section 2.1.

Now that ontologies are being increasingly adopted in information systems and that the Ontology Engineering field is facing the same problems that Software Engineering did, it is clear that ontology engineering tasks may also benefit from the application of common software engineering practices. Therefore, Section 2.2 is focused on the state of the art in Software Engineering: Section 2.2.1 offers the insights of the main testing techniques, Section 2.2 .2 describes the main works that integrate verification testing and development methodologies, and Section 2.2 .3 presents the most important conformance testing approaches; finally Section 2.2.4 is oriented to describe the metrics proposed to support the software life-cycle that are extracted from artefacts related to software testing, such as requirements or test cases.

Section 2.3 is oriented to the relevant state of the art in Ontology Engineering. First, Section 2.3.1 introduces how well-known methodologies and lightweight methodological approaches in the Ontology Engineering field deal with the verification activity and the specification of requirements. Section 2.3 .2 describes methodologies and lightweight methodological approaches that propose tests extracted from the requirements to address the ontology verification activity. Next, Section 2.3 .3 describes the work on requirements analysis for tests definition and Section 2.3.4 describes the insights of some testing approaches for verifying requirements, which is the main issue addressed in this 
thesis. After that, Section 2.3.5 provides an overview of the main tools for executing queries on ontologies and that can be used for ontology verification, and Section 2.3.6 introduces the work on integrating testing within the ontology development process. Section 2.3.7 focuses on ontology metrics related to the artefacts generated during the testing process whose aim is to monitor the ontology life-cycle.

Section 2.4 concludes the chapter with a summary of the main gaps in the existing ontology testing approaches, tools and metrics for ontology verification and introduces how this thesis can contribute to advance the state of the art.

\subsection{Terminology}

This section gathers the definitions of ontology evaluation and testing that will be used throughout this thesis. Bearing in mind that ontologies are part of software products and sometimes the adoption of software practices can be helpful for Ontology Engineering, this section includes term definitions taken from the Software Engineering field. Moreover, such definitions taken from the Software Engineering field have been used as inspiration for the evaluation-related definitions in Ontology Engineering.

The "ISO/IEC 25040:2011" standard (ISO, 2011b) introduces a set of definitions related to the evaluation of software systems:

- Evaluation. Systematic determination of the extent to which an entity meets its specified criteria.

- Evaluation method: Procedure describing actions to be performed by the evaluator in order to obtain results for the specified measurement applied to the specified product components or on the product as a whole.

Based on these evaluation-related definitions, the "ISO/IEC 25066:2016" standard (ISO, 2016) presents the following definition related to conformance, which is a type of evaluation of a system:

- Conformity assessment (also known as conformance). Demonstration that specified requirements relating to a product, process, system, person or body are fulfilled. 
Moreover, the "ISO/IEC/IEEE 29119-1:2013" standard (ISO/IEC/IEEE, 2013) defines the term testing and the different types of testing, which are used for the evaluation of a system:

- Testing. Set of activities conducted to facilitate discovery and/or evaluation of properties of one or more test items.

- Verification. Confirmation, through the provision of objective evidence, that specified requirements have been fulfilled in a given work item.

- Validation. Demonstrates that the work item can be used by the users for their specific tasks.

All these previous definitions were oriented to the Software Engineering field. The NeOn Methodology (Suárez-Figueroa et al., 2013), uses these previous definitions to adopt the evaluation concepts into the Ontology Engineering field by describing the following terms:

- Ontology evaluation. It refers to the activity of checking the technical quality of an ontology against a frame of reference.

- Ontology verification. It is the ontology evaluation activity that compares the ontology against the ontology requirements specification document (ontology requirements written as competency questions or statements), ensuring that the ontology is built correctly (in compliance with the ontology requirement specification). It answers the question "Are you producing the ontology right?".

- Ontology validation. It is the ontology evaluation activity that compares the meaning of the ontology definitions against the intended model of the world aiming to conceptualise. It answers the question "Are you producing the right ontology?".

Given these definitions related to Software Engineering and Ontology Engineering, this thesis also proposes the following definitions, which will be used along with this thesis:

- Ontology verification testing. It refers to the activity of executing tests with the intent of verifying whether ontology requirements are satisfied by an ontology. 
- Ontology conformance testing. It refers to the activity of executing tests with the intent of verifying whether specified requirements related to a standard are fulfilled.

\subsection{Related work in Software Engineering}

This section summarises the main testing techniques in the Software Engineering field (Section 2.2.1), as well as how verification testing methods can be integrated as an integral part of the development methodologies (Section 2.2.2). Additionally, this section presents the conformance testing approaches for Software Engineering (Section 2.2.3). Finally, it also presents a set of metrics that are extracted from artefacts generated during the software development process to monitor the software life-cycle, such as the ontology implementation, requirements and test cases (Section 2.2.4.

\subsubsection{Software testing techniques}

Testing is an essential activity in Software Engineering and is widely used in industry for quality assurance. It observes the execution of a software system to validate whether it behaves as intended and to identify potential malfunctions. By directly analysing the software in execution, it provides a realistic feedback of its behaviour (Bertolino, 2007). This section summarises the most used testing techniques in the Software Engineering field, which can be used for the verification of a system.

\subsubsection{Unit testing}

In Software Engineering, the idea of unit testing (Beck, 1999) was introduced to counter the complexities of modern software engineering efforts. Unit testing means testing the smallest separate module in the system (Runeson, 2006).

Unit tests are meant to facilitate the development of program modules or units, and to ensure the interplay of such units in the combined system. It results in code that is easier to refactor and simpler to integrate, and that has a formalised documentation. Unit tests can be added incrementally during the maintenance of a piece of software. They are structural tests, or white-box based, but developers rarely measure their completeness in terms of structural coverage. 
Unit tests in Software Engineering became popular with the object-oriented language Smalltalk $3^{3}$ and still to this day remain focused on languages with strong possibilities to create smaller units of code.

\subsubsection{Acceptance testing}

Acceptance tests (Miller and Collins, 2001) in a software development process represent the customer's interests. They aim at improving the customer confidence on the correct behaviour of the application and the satisfaction of the needed features. Thus, preserving those tests, running them frequently, and amending them as requirements change, proves that the product still satisfies the customer's needs.

Acceptance tests provide several benefits to the software development team: (1) they capture user requirements in a directly verifiable way; (2) they measure how well the system meets those requirements; and (3) they provide a ready-made definition of how complete the system is according to the customer's needs.

\subsubsection{Keyword-driven testing}

Keyword-driven testing (Utting and Legeard, 2010 ) is a testing approach that uses keywords in the tests. The idea behind keyword-driven testing is to express each test as abstractly as possible, but still precise enough to be executed and interpreted by a test execution tool. The set of tests can be based on customer specifications, i.e., on software requirements.

This method separates the task of the test implementation from the software development, i.e., separation of implementation work and test definition. Therefore, maintainability increases because of the separation between the test definition and the functionality implementation.

Keywords control the processing and are crucial in this approach. Each keyword corresponds to a fragment of a test script (i.e., the adapter code), which allows the test execution tool to translate a sequence of keywords and data values into executable tests (Wieczorek et al., 2012). Tests can be adapted to a new version of the system under test by updating the test scripts associated with a few keywords.

$\sqrt[3]{\text { http://wiki.c2.com/?SmalltalkUnit }}$ 


\subsubsection{Software verification testing integrated into software develop- ment methodologies}

In addition to these testing techniques, in the Software Engineering field some development methodologies integrate the testing process as an integral part of the methodology. This is the case of the Test-driven development and the Behaviour-driven development approaches, which are described in the following sections paying special attention to how they manage the verification of the resultant product.

\subsubsection{Test-driven development}

Test-driven development (TDD) (Beck, 2003) is a software strategy that requires writing automated tests before developing functional code in small, rapid iterations.

Traditionally, unit testing occurred after developers coded the unit. The unit tests might be written by the same programmer or by a designated tester. With TDD, the programmer writes the unit tests before the code under test. As a result, the programmer can immediately execute the tests after they are written (Janzen and Saiedian, 2005).

TDD is a practice that instructs developers to write new code only if an automated test has failed, and to eliminate duplication. The ultimate goal of TDD is to write "clean code that works". Among the drawbacks of TDD, the developer must maintain both the production code and the automated tests. Figure 2.12 summarises the steps that should be carried out during a TDD project.

A sub-type of this TDD methodology is the Acceptance test-driven development (ATDD) (Koskela, 2007), which emphasises writing software requirements in form of acceptance tests prior to the code under test.

\subsubsection{Behaviour-driven development}

Behaviour-driven development (BDD) is an agile software development approach, whose core principle states that "business and technology people should refer to the same system in the same way". Therefore, a common language is needed for specifying system behaviours, which allows: (a) customers to specify the requirements from a business perspective, (b) business analysts to attach concrete examples that clarify the system 


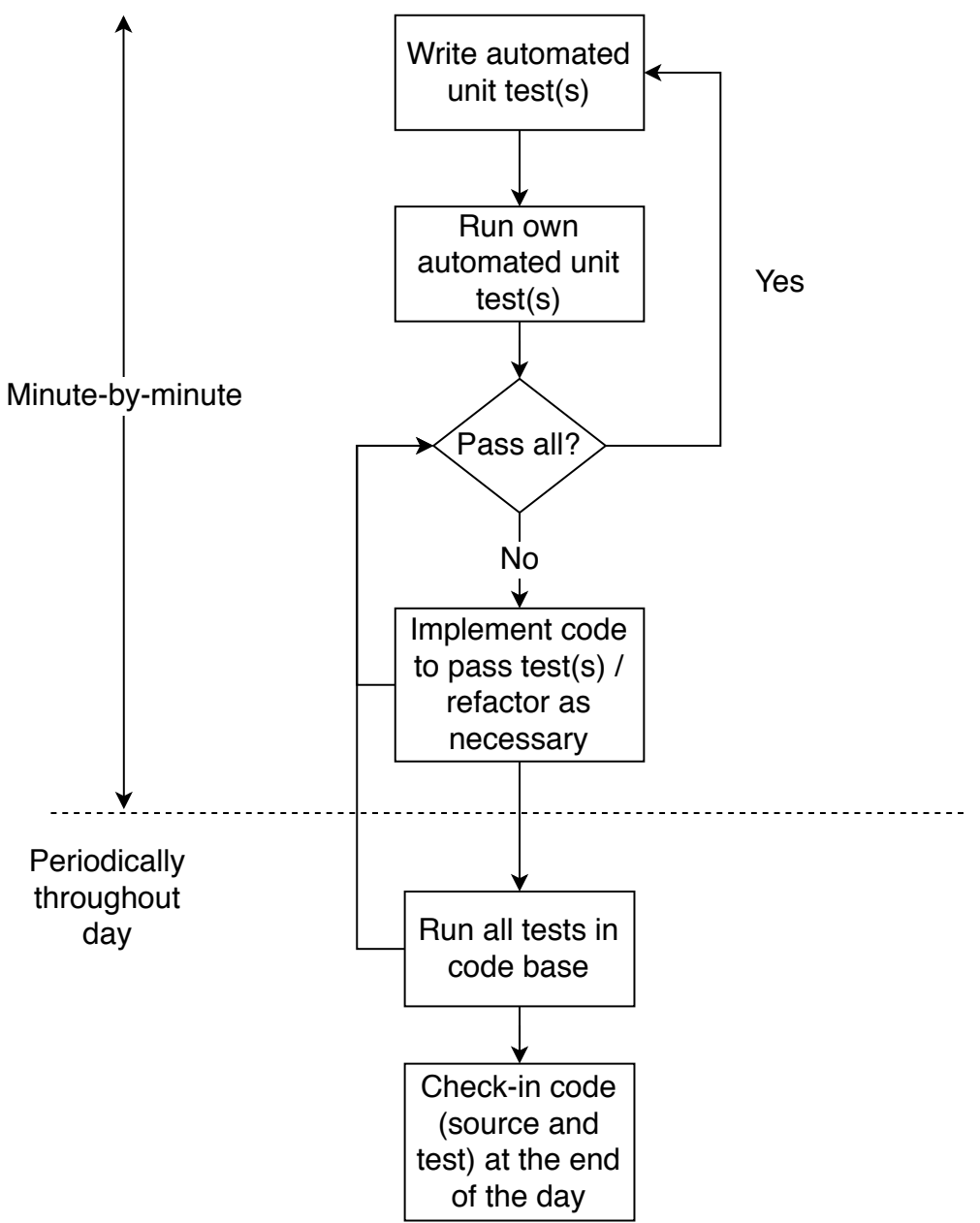

Figure 2.1: Test-driven development steps (Bhat and Nagappan, 2006)

behaviour, and (c) developers to implement the required system behaviour in a TDD manner (Lazar et al., 2010).

The second core principle of BDD states that "any system should have an identified, verifiable value to the business". It is needed to identify when a behaviour is satisfied. If the behaviour is described using executable scenarios (executable tests) then the software can be automatically verified through successful passing of such tests.

BDD is generally regarded as the evolution of TDD. BDD is focused on defining fine-grained specifications of the behaviour of the targeting system, in a way that they can be automated. The main goal of BDD is to get executable specifications of a system. In BDD, tests are clearly written and easily understandable, since it provides 
a particular language, e.g., Gherkin (Wynne et al. 2017), that helps stakeholders to specify the behaviours, which are mapped to tests directly. The template for specifying new behaviours is the following (Solis and Wang, 2011):

Scenario 1: [Scenario Title]

Given [Context]

And [Some more contexts] ...

When [Event]

Then [Outcome]

And [Some more outcomes] ...

In these templates the descriptions in square brackets should be written in the language defined in the project in order to be able to be mapped to tests.

\subsubsection{Software testing conformance methods}

Software conformance testing refers to a type of verification testing that tries to verify whether a product, process, computer program or system meets a defined standard specification (Moseley et al., 2003). Ensuring conformance to standards plays an important role in systems across many domains and several standardisation bodies have presented their own conformance testing approaches (Graydon et al., 2012).

This section presents a summary of the most relevant conformance testing approaches, which are mostly oriented to software and industry products.

\subsubsection{ISO/IEC 9646}

ISO/IEC 9646 (ISO, 1994) is a multi-part international standard which specifies a general methodology for conformance testing of products to Open Systems Interconnection specifications. However, the concepts for testing their implementations have a broader applicability and can also be used in testing of other kinds of protocol systems. This standard aims at providing a methodology for verifying whether requirements of a standard are satisfied by a system. The standard also defines a testing language called TTCN (Kristoffersen and Walter, 1996), which is a tree and tabular notation for a formal description of test cases whose language is completely independent of technology, operating system and implementation domain. This testing language follows the same idea of keywords that the Keyword-driven testing method presented in Section 2.2.1.3. 
in the sense that both use a controlled testing syntax to define tests that can be executed on a system.

The process of conformance testing in this ISO/IEC norm begins with the collation and categorisation of the features and options to be tested into a tabular form which is normally referred to as the implementation conformance statement (ICS). All implemented capabilities supported by the implementation under test are listed by the implementer in the ICS, so that the tester knows which options have to be tested.

The next step is to collect the requirements from the standard specification related to the features to be tested. For each requirement, one or more tests should be identified and expressed in the form of test purposes (TP), which describe a well-defined objective of testing. The TP describes in plain language the actions required to reach a verdict on whether an implementation passes or fails the test. Then, the tests are classified into a number of groups which will provide a structure to the overall test suite structure (TSS). The test cases are combined into an abstract test suite (ATS) using the TTCN testing language. In this scenario test suites should be abstract meaning that they are developed independently of any implementation and, therefore, reusable. With this, the same tests can be executed on multiple systems, allowing to check whether a particular system satisfies the requirements of a third-party standard.

The test suite is then verified against a number of implementations under test for correct operation according to some agreed procedures. An implementation extra information for the test (IXIT), which typically contains information about the physical setup and connection of the test, associated with the ATS should be produced to help to execute conformance testing. The results of this verification process are documented in the conformance test report. Figure 2.2 summarises these activities.

This methodology is focused on the definition of the activities needed to define tests extracted from the requirements of a standard, and to the execution of such tests on a system. With this methodology, tests obtained from the requirements of third-party standards can be executed on multiple systems.

\subsubsection{ETSI}

The ETSI conformance testing specifications are developed according to the method described in the ISO/IEC 9646. The specification provided by ETSI (Moseley et al. 2003 ) is also focused on several artefacts, previously defined in the ISO/IEC 9646, 


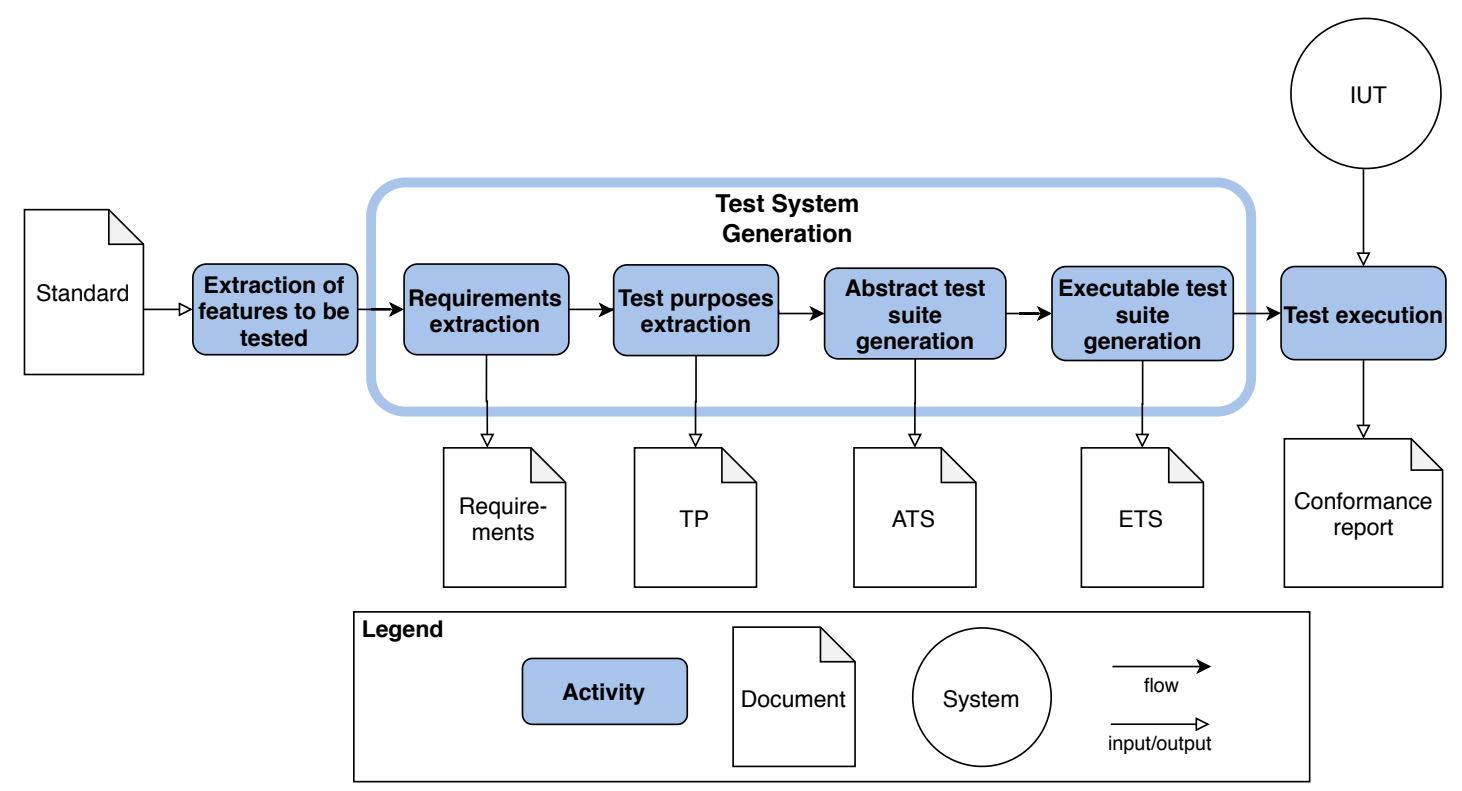

Figure 2.2: Summary of ISO/IEC 9646 activities for conformance testing

namely, the Implementation Conformance Statement (ICS), the Implementation eXtra Information for Testing (IXIT), the Testing Purposes (TP), the Abstract Test Suite (ATS) and the Executable Test Suite (ETS). Figure 2.3 shows the development of the test specification process.

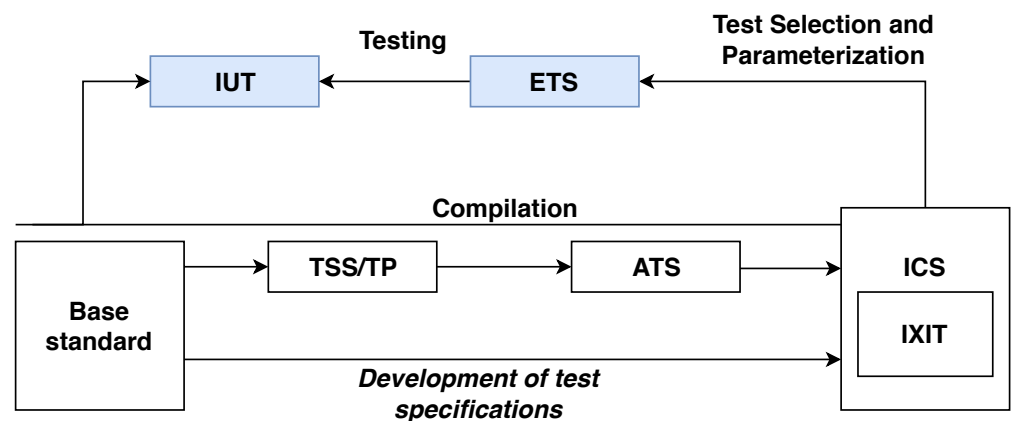

Figure 2.3: Development of ETSI test specifications (Moseley et al. 2003)

Similarly to the ISO/IEC 9646, the ATS represents the entire collection of test cases. In the ETSI conformance testing method, each test case specifies the detailed coding of the TP written using the standardised test specification language TTCN-3 (Grabowski et al. 2003), which is an updated version of the TTCN testing language. The ETS can be implemented from the ATS using the TTCN compilers available on many testing 
platforms.

All the test specifications developed by ETSI are available online and can be searched and downloaded via the ETSI Work Programme application 4 These specifications include a description of the tests in a human-readable format as well as the executable tests written following the TTCN language, so that other developers can execute them on their systems to check conformance.

\subsubsection{W3C}

The conformance testing approach presented by the $\mathrm{W} 3 \mathrm{C}$ is focused on the requirements and good practices for including conformance in the W3C specifications (Dubost et al. 2005), emphasising that it is required to have a conformance clause in order to develop successful interoperability of implementations.

The $\mathrm{W} 3 \mathrm{C}$ proposes requirements such as the addition of conformance clauses in every specification, as well as the identification of which conformance requirements are mandatory, recommended and optional. Additionally, it also requires to use a consistent style for conformance requirements and explain how to distinguish them. An example of detailed conformance clauses following the W3C guidelines is the Scalable Vector Graphics (SVG) 1.1 (Dahlström et al., 2011) specification, which describes all the requirements that should be fulfilled.

Regarding good practices, the W3C proposes the definition of the specification's conformance model and the specification of how to distinguish normative from informative content, as well as the description of the wording for conformance claims.

The $\mathrm{W} 3 \mathrm{C}$ is focused on how to publish the conformance clauses on a system specification, rather than on defining a homogeneous method for defining tests to analyse whether a system verifies the requirements of a particular standard.

\subsubsection{Software metrics to support the software life-cycle}

In a software development process it is not enough to monitor a single artefact, i.e., the software product. Nevertheless, the results of the testing process should be analysed, as well as the artefacts associated with it and the process itself, in order to guarantee the quality of the development. In Software Engineering, metrics related both to the

\footnotetext{
${ }^{4}$ https://portal.etsi.org/webapp/WorkProgram/SimpleSearch/QueryForm.asp
} 
artefacts and to the development process have been of interest for years both from the research perspective and in the industrial practice, providing several benefits to software development, such as defect prediction (Moser et al. 2008) or quality assessment Kan, 2002 .

However, the most significant benefit of these metrics is the ability to provide information to support managerial decision making during the software development process. Since software metrics are extracted not only from the software implementation, but also from other artefacts such as the requirements specification or the test cases, they allow to monitor not only the source code, but also the requirements specification and the test cases. Therefore, these metrics provide software developers with more information about the status of the software product and its development process.

The first software metric dates back to the mid-1960's when the Lines of Code metric ( $L O C$ or $K L O C$, for thousands of lines of code) was used as the basis for measuring programming productivity and effort (Fenton and Neil, 2000). In the late 1960's LOC was also used as the basis for measuring program quality; it was used to calculate derived metrics such as the number of defects per KLOC. The critical assumption of these metrics is that it considers the size of the program as the key aspect to measure quality and effort.

Nowadays, software metrics are divided into two categories, namely, product metrics, which are used to measure internal attributes of a software system, and process metrics which are used to obtain measurements about the software process (Sommerville, 2010). Product metrics can be related to the different artefacts produced in the software development process, such as the source code, the requirements specification or the user documentation. Process metrics are usually related to the software life-cycle, although there are also process metrics which are associated with different artefacts, such as the requirements specification. This section presents a summary of the most relevant metrics for Software Engineering, classified regarding whether they are product and process metrics and from which artefacts they are extracted.

\subsubsection{Source code product metrics}

Fenton and Bienman (Fenton and Bieman, 1997) present the COCOMO 2.0 model (Boehm et al., 1995) for predicting project cost, effort and calendar time. COCOMO 2.0 provides different effort estimating models based on the stage of the development 
of the project. As inputs, COCOMO 2.0 requires the level of information available to the user at that time. Therefore, during the earliest conceptual stages of a project, the models use object points, e.g., screens, reports and modules of the language, to compute effort. During the early design stages it uses as input the unadjusted function points, which represent the functionally size of the software implementation based on the logical view of an application. Finally, once an architecture has been selected, LOC is the input to the COCOMO 2.0 model.

Dealing with the same topic, Kan's work (Kan, 2002) includes the Changed source instructions metric (CSI), which counts the new and changed source instructions in a software implementation. Another product metric presented in this work is the Problems per user month (PUM), which represents the total number of problems that customers reported for a time period in addition to the total number of license-months of the software during the period.

In addition to this, Sommerville (Sommerville, 2010) introduces several product metrics related to the source code, such as the Fan-in, Fan-out and Depth of conditional nesting metrics. Fan-in is a measure of the number of functions or methods that call another function or method and Fan-out is the number of functions or methods that are called by a given function or method. Depth of conditional nesting measures the depth of nesting of "if" statements in a program.

\subsubsection{Requirements specification product metrics}

Concerning the metrics for the requirements specification product, Davis and colleagues (Davis et al. 1993) define a set of 18 product metrics to measure the software requirements specification (SRS) quality, which is associated with attributes of the requirements specification document. The authors provide formulas for the following metrics: (1) Unambiguity, which measures the percentage of requirements that have been interpreted in an unique manner by all of its reviewers; (2) Completeness, which measures different aspects related to the completeness of a SRS; (3) SRS correctness, which calculates the percentage of the requirements specification that has been validated; (4) Understandability, which measures the percentage of requirements for which all reviewers thought they understood; (5) SRS verifiability, which considers the cost and time necessary to verify a requirement; (6) SRS internal consistency, which calculates the percentage of requirements that are not in conflict; (7) SRS external consistency, which 
measures the percentage of requirements that are consistent with all other documents; (8) SRS conciseness, which counts the number of pages in the requirements specification document; (9) SRS design independence, which measures the percentage of possible solution systems that are eliminated by adding the overly constraining requirements; (10) SRS annotated with relative importance, which calculates the percentage of requirements that are annotated with their importance; (11) Non redundant SRS, which calculates the percentage of requirements that are stated more than once; and (12) Traced, which measures the percentage of requirements that are traced.

Costello and Liu (Costello and Liu, 1995) were focused on providing metrics related to requirements, with the aim of monitoring and better controlling the requirements engineering process. The key goal of Costello and Liu was to use of requirements completeness metrics to quantify the status of the requirements specification development via object information. Additionally, they also quantified the results of specification inspections in requirements inspection and defect density metrics. This allows to separate collected requirements from data collected via subjective thought processes.

Fenton and Bieman (Fenton and Bieman, 1997) point out that the length of the requirements specification might be related to the complexity of the code and, therefore, it is useful to measure it. An example of this type of metrics is the Specification weight, formerly the Bang metrics. It involves two metrics: (1) Function bang metric, which is based on the number of functional primitives in a data-flow diagram, and (2) Data bang metric, which measures the number of entities in the entity-relationship model.

Iqbal and colleagues (Iqbal et al. 2012) propose a set of metrics to evaluate the effectiveness of the requirements. Based on their experience of gathering requirements for a number of software projects, Iqbal and colleagues propose a set of metrics that can be used during the entire course of action of the requirement engineering process and offer a blend of quantitative measures to judge the performance and quality of the requirement gathering and elicitation phase. They also proposed a criterion against each of these metrics which refers to a recommended level to confirm the validity of a requirement. However, this can be slightly changed depending on the nature of the project and on the needs of the organisation.

The set of requirements metrics presented by Iqbal and colleagues includes, among others, the Misinterpreted requirement metric, which checks the percentage of the requirements that have been misinterpreted during the elicitation phase, and the Cor- 
rectness metric, which measures the percentage of requirements which are correctly validated. If the result of the Misinterpreted requirement metric is higher than $5 \%$, then the elicitation process needs to be redone. Additionally, if the Correctness metric is less than $80 \%$, then it is assumed that the correctness cannot be verified.

\subsubsection{Test case product metrics}

Fenton and Bieman (Fenton and Bieman, 1997) also describe several test case product metrics, such as Branch coverage, which finds the set of paths such that every edge lies on at least one path, and the Test effectiveness ratio, which it is obtained by dividing the number of tests exercised at least once and the total number of tests.

\subsubsection{Software documentation product metrics}

Regarding the software documentation product metrics, Sommerville (Sommerville, 2010) presents the Fog index (Gunning, 1952), which indicates the average length of words and sentences in documents and is used in software engineering to measure the readability of the user documentation. The higher the value of a document's Fog index, the more difficult the document is to understand.

\subsubsection{Process metrics}

Process metrics, which calculate measurements related to the development process, can be extracted from the development process itself, as well as from different artefacts generated during the development process.

Costello and Liu (Costello and Liu, 1995) propose a collection of process metrics related to requirements that includes Requirements volatility, Requirements fault density or Requirements traceability. Requirements volatility refers to changes and reasons for changes to requirements; Requirements fault density indicates the number of requirement faults that are initially detected during test execution or during the analysis of the tests after being executed; and Requirements traceability indicates the degree to which a development organisation maintains accountability for meeting requirements at each stage of the life cycle via a requirements traceability matrix.

Rahman and Devanbu (Rahman and Devanbu, 2013) gathered a set of file-based process metrics which can be applied to code, requirements, tests or documentation. 
These metrics include: (1) COMM, which measures the number of commits made to a file; (2) $A D E V$, which is the number of developers who changed the file; (3) DDEV, which is the cumulative number of distinct developers who contributed to this file up to this release; (4) $A D D$ and $D E L$, which are the normalised (by the total number of added and deleted lines) added and deleted lines in the file; (5) $O W N$, which measures the percentage of lines authored by the highest contributor of a file; (6) MINOR, which measures the number of contributors who authored less than $5 \%$ of the code in a file; (7) OEXP, which measures the experience of the highest contributor of a file using the percent of lines the contributor authored in the project at a given point in time; and (8) EXP, which measures the geometric mean of the experiences of all the developers.

\subsection{Related work in Ontology Engineering}

This section includes a summary of the ontology verification methods based on the specification of requirements (Section 2.3.1) and based on the definition of tests from such requirements (Section 2.3.2) within well-known ontology development methodologies. These methodologies show how the ontology verification methods can be integrated as an activity within a comprehensive process and how the verification results can be used in the development process. This section also includes the work related to the analysis of requirements for the definition of tests (Section 2.3.3) and the most significant work on ontology testing approaches and tools for ontology verification (Section 2.3.4), on querying approaches for testing ontologies (Section 2.3.5), and on the testing approach integrated within the development process (Section 2.3.6). Figure 2.4 summarises these methodologies and approaches and shows the relation between them. Finally, ontology metrics to support the ontology life-cycle and to monitor the ontology development are also described (Section 2.3.7).

\subsubsection{Requirements specification in ontology development method- ologies}

This section provides an overview of the main methodologies and lightweight methodological approaches in the Ontology Engineering field that introduce the ontology verification activity and the specification of requirements (written mostly as competency 





questions). In the following sections a brief description of each methodology is provided, together with a description of how it deals with ontology testing for ontology verification.

\subsubsection{Grüninger and Fox}

The methodology proposed by Grüninger and Fox (Grüninger and Fox, 1995) provides a framework for evaluating the adequacy of these ontologies by means of competency questions. It is based on the generation of common sense models, which are models that have the ability to deduce answers to queries that require relatively shallow knowledge of the domain. The steps included in such methodology are depicted in Figure 2.5.

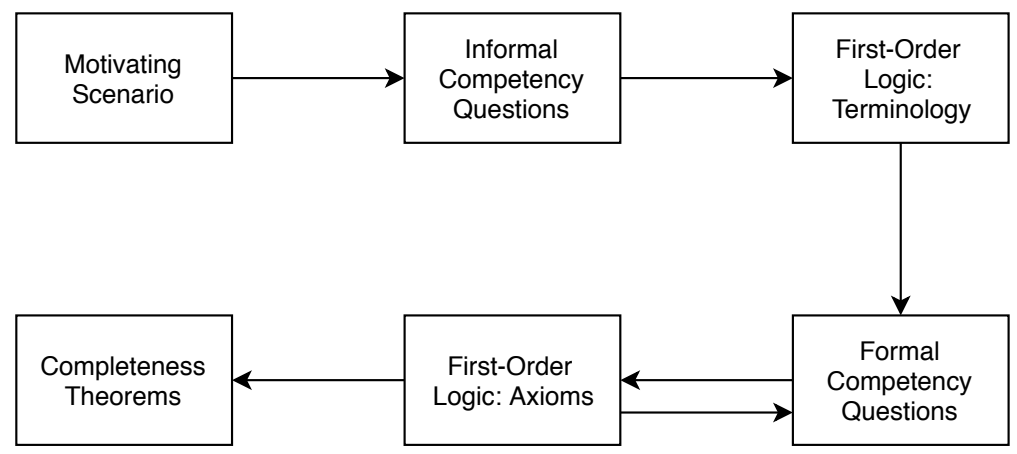

Figure 2.5: Procedure for ontology design and evaluation (Grüninger and Fox 1995)

The approach begins with the presentation of some motivating scenarios that often have the form of story problems or examples which are not adequately addressed by existing ontologies. Given the motivating scenario, a set of queries will arise which place demands on an underlying ontology. These queries can be considered to be requirements that are in the form of questions that an ontology must be able to answer. These are the informal competency questions, since they are not yet expressed in the formal language of the ontology.

The next step is to define the terminology of the ontology, including its objects, attributes and relations. This terminology should be specified by using First Order Logic (Smullyan, 2012).

Once the competency questions have been posed informally and the terminology of the ontology has been defined, the competency questions are defined formally as an entailment or consistency problem with respect to the axioms in the ontology. 
After that, axioms expressed in First Order Logic are included in order to specify the definitions of the terms and constraints. This is an iterative process guided by the formal competency questions. If the proposed axioms are insufficient to represent the formal competency questions, additional objects or axioms must be added to the ontology until it is sufficient. Finally, the competency questions are tested by proving completeness theorems, with the aim of verifying that they are satisfied by the ontology.

This work introduced the notion of competency questions, which are widely used for extracting ontology requirements and for testing ontologies and that will be used throughout the rest of this thesis. Furthermore, this work represents the first steps towards the verification of ontologies based on these competency questions. This methodology is focused on providing an outline on how to write competency questions and how to verify an ontology based on them.

\subsubsection{ON-TO-KNOWLEDGE}

The On-To-Knowledge methodology (Staab et al., 2001) lies in the application-oriented development of ontologies. As it is shown in Figure 2.6, it includes five phases, namely: (a) Feasibility study, (b) Ontology kickoff, (c) Refinement, (d) Evaluation, and (e) Maintenance.

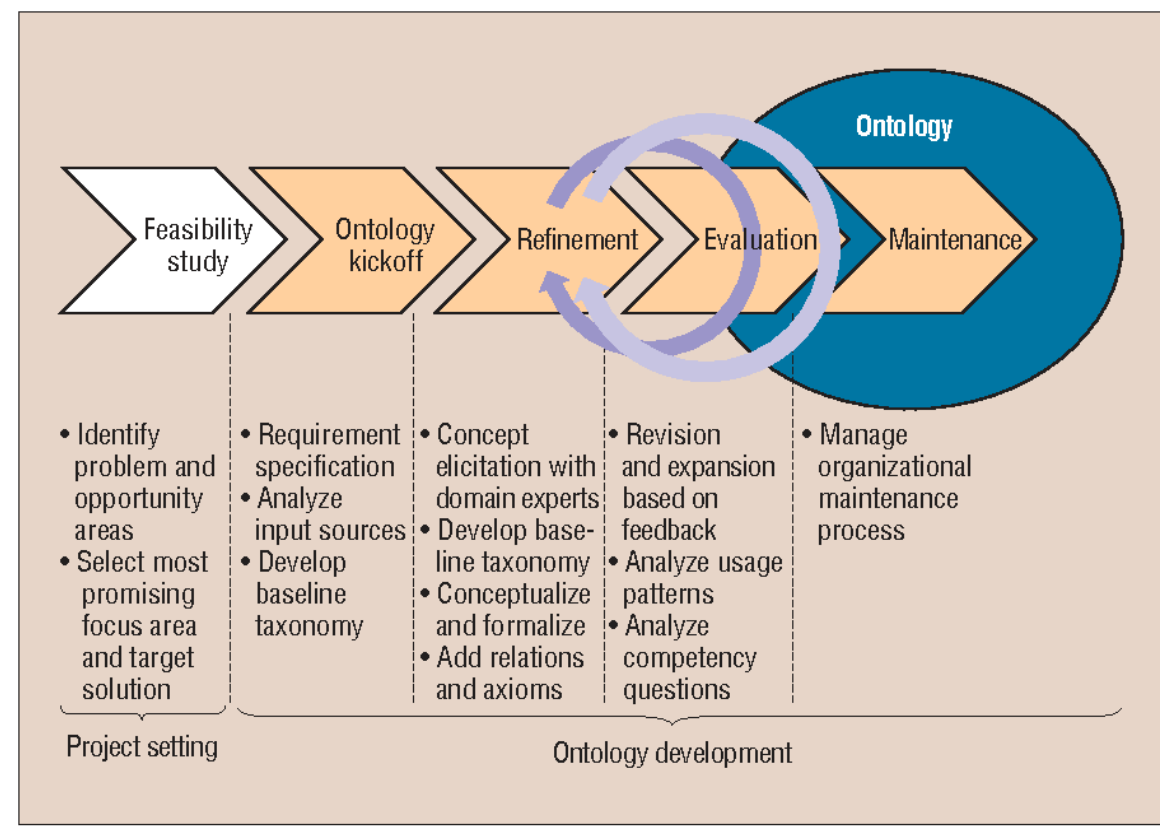

Figure 2.6: On-To-Knowledge development phases (Staab et al. 2001) 
During the ontology kickoff phase, ontology engineers should collect user requirements written as competency questions to provide an overview of possible queries to the system that can indicate the scope and content of the domain ontology. Next, during the evaluation phase, ontology engineers verify whether the target ontology satisfies the ontology requirements specification document and whether the ontology supports or answers the competency questions analysed in the kickoff phase of the project. During this evaluation phase new requirements that should be handled by the ontology can come up.

It can be observed in Figure 2.6 that there are loops between the Refinement and the Evaluation phases, which shows that it is an iterative process. An ontology engineer might need to perform several cycles until finishing the ontology.

ON-TO-KNOWLEDGE defines the evaluation activity and how to perform it, emphasising the use of the requirements to verify the ontology. However, the definition of the tests is out of scope of this methodology.

\subsubsection{EXtreme Ontology}

The eXtreme ontology method (Hristozova and Sterling, 2002) aims at building lightweight ontologies, applying the principles of eXtreme Programming (XP) Beck and Gamma, 2000). The process includes the following steps:

1. To fetch the requirements of the system. The collected data will be used to create a baseline ontology.

2. To define competency questions. They are domain-specific and provided by the user. Competency questions provide the base for extracting the ontology concept and are also used for completeness and for the validation of the requirements.

3. To run validation tests. The validation tests check whether the competency questions could be answered using the existing ontology.

4. To run redundancy tests. Redundancy tests are run to check existing classes, attributes or relationships with similar or matching names.

5. To plan. The planning process includes the estimation of the cost and time for building the developed ontology. 
6. To integrate. During the process of integration it is needed to explore how the ontology will react with the other modules of the system.

7. To run acceptance tests. This type of test proposed by the authors is used to evaluate those concepts that are ontology commitments provided by the users and it is not oriented to the ontology structure. These tests evaluate the expressiveness of the ontology that is required to represent the questions and to characterise their solutions.

This methodology is focused on capturing system requirements and it considers the intended use of the ontology by analysing the provided competency questions. It mentions the definition of tests to check the competency questions; however, it does not provide any technique to define them.

\subsubsection{Tests definition in ontology development techniques}

In the following sections a brief description of the methodologies that propose the definition of tests from ontology requirements is provided, together with a description of how they deal with ontology testing for ontology verification.

In the 2000's, Vrandečić and Gangemi presented the notion of unit testing for ontologies (Vrandečić and Gangemi, 2006) by adapting the idea of unit tests from Software Engineering. In Software Engineering, unit tests are easily manageable tests for small parts of a program. Vrandečić and Gangemi tried to adapt this idea of unit testing for ontologies by proposing different approaches, among which is the formalisation of competency questions. This approach for unit testing consists of formalising the competency questions in a query language and formalising the expected answers as well. This allows a system to automatically check if the ontology meets the requirements stated with the competency questions. Therefore, since in this thesis the verification of ontologies is focused on the testing of requirements (which can be written as competency questions), the tests presented in the following sections can be considered as unit tests.

\subsubsection{1 eXtreme Design}

The eXtreme Design (XD) approach has been first introduced in the NeOn project (Suárez-Figueroa et al., 2009a) and then revisited by (Presutti et al., 2009). It is an approach based on the application, exploitation, and definition of ontology design 
patterns (ODPs) for solving ontology development issues. XD is partially inspired by the software engineering practice eXtreme Programming (XP) (Beck and Gamma, 2000). However, their focus is different: where XP diminishes the value of careful design, this is exactly where XD has its main focus. However, they also have similarities; XD is test-driven, and applies the divide-and-conquer approach as well as XP does, and both adopt pair design, as opposed to pair programming.

XD follows a set of principles, which are inspired by the XP approach, namely: (a) customer involvement and feedback; (b) customer stories, competency questions, and contextual statements; (c) iterative development; (d) test-driven design; (e) ontology design pattern reuse and modular design; (f) collaboration and integration; (g) taskoriented design; and (h) pair design. The overall process is depicted in Figure 2.7.

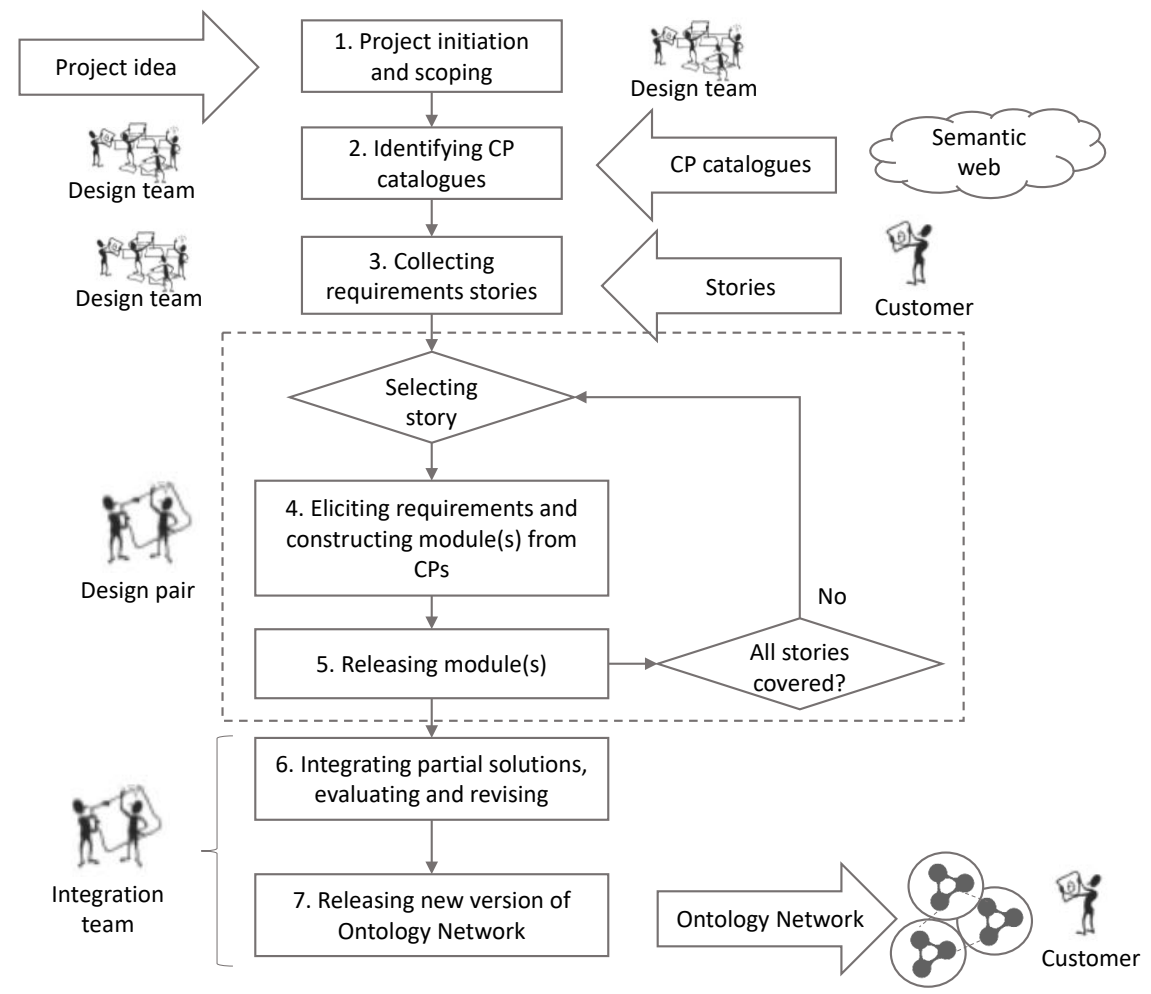

Figure 2.7: The overall XD process (Presutti et al. 2009)

In this methodology, the testing activity is used as an integrated means for completing the modules. Stories, competency questions, reasoning requirements, and contextual statements are used in order to develop unit tests, e.g., competency questions can be 
transformed into SPARQL queries. In this sense, by deciding how the query should be formed, a developer is actually partly designing the model.

This methodology goes further than the previously described methodologies, since it introduces SPARQL queries as a type of test for verifying the competency questions of an ontology to be developed.

\subsubsection{SAMOD}

SAMOD (Peroni, 2016) is an agile methodology for the development of ontologies by means of small steps of an iterative workflow that focuses on creating well-developed and documented models. It includes three steps, which are summarized in Figure 2.8.

1. To define a new test case. Given a motivating scenario, ontology engineers and domain experts should produce a set of informal competency questions. From them, ontology engineers should create the modelet, which is a stand-alone model describing a particular aspect of the domain. Besides, competency questions are translated into SPARQL queries to verify whether the modelet covers the related motivating scenario. Finally, ontology engineers should create an exemplar dataset that formalises all the examples introduced in the motivating scenario according to the modelet.

2. To merge the current model with the modelet. At this stage, ontology engineers should merge the modelet with the current model, i.e., the version of the final model released at the end of the previous iteration.

3. To refactor the current model. In the last step, ontology engineers work to refactor the current model by reusing existing knowledge, documenting it and enriching the current model by using all the capabilities offered by OWL 2 .

Test cases are an integral part of this methodology and represent a complete documentation of a particular aspect of the domain described by the model. Test cases include natural language descriptions (the motivating scenario and the competency questions) as well as the formal implementation of exemplar data and possible ways of querying the data compliant with the model (the set of SPARQL queries). Similarly to the eXtreme Design methodology, SAMOD proposes the use of SPARQL queries based on the competency questions to test the developed ontology. 


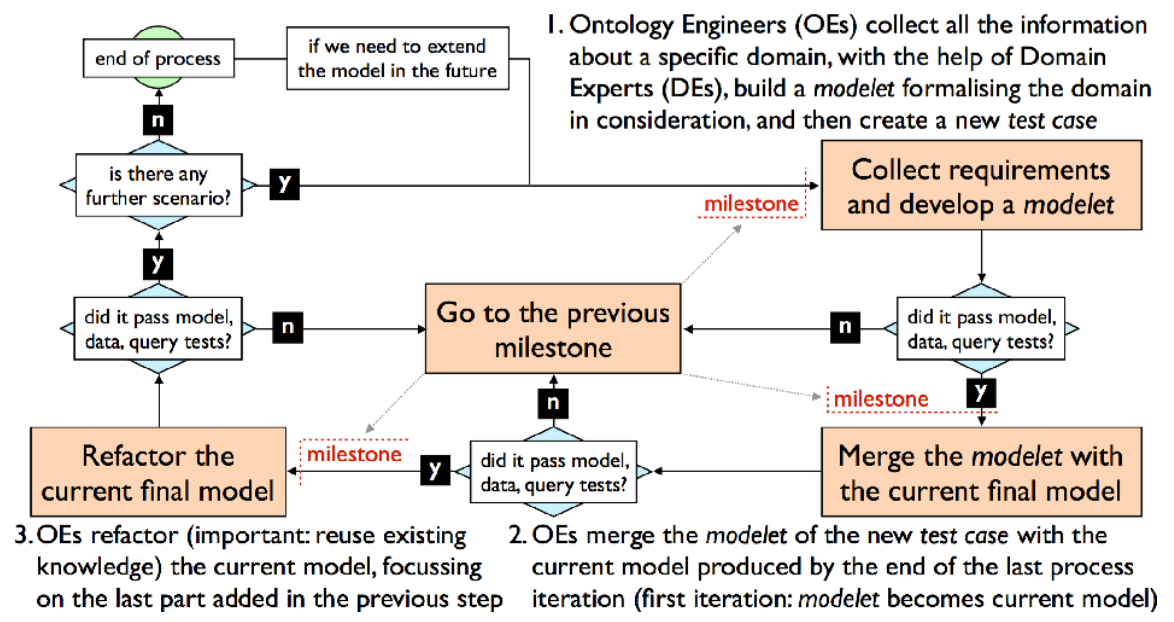

Figure 2.8: A brief summary of SAMOD (Peroni, 2016)

\subsubsection{Requirements analysis for ontology verification testing}

Several works are also focused on the analysis of how requirements are specified, with the ultimate goal of helping their translation into formal queries that can be executed on an ontology.

These works are not focused on providing a process for automatically translating natural language requirements into formal queries, but on providing lists of linguistic patterns that can be used to make such translation.

\subsubsection{Ren and colleagues' requirement analysis}

The work presented by Ren and colleagues (Ren et al. 2014) focuses on Competencyquestion-driven authoring. In this work the authors use natural language processing and patterns to analyse requirements written in the form of competency questions with the ultimate goal of automatically obtaining SPARQL queries to test an ontology. Therefore, this analysis of requirements can be used for the definition of unit tests to verify an ontology.

The authors analysed a set of 75 competency questions to identify the patterns to which they belong. In order to represent the commonality and variability of different competency question patterns, they employed the feature-based modelling method and describe different competency question patterns with regards to a set of features: 
- Question Type, which determines the kind of answers presented when answering the competency question, such as selection questions, binary questions and counting questions.

- Element Visibility, which indicates whether the modelling elements, such as the class expressions and property expressions are explicit or implicit in the competency question.

- Question Polarity, which determines if the question is asked in a positive or negative way.

- Predicate Arity, which indicates the number of arguments of the main predicate manner.

- Relation Type, which indicates the kind of relation for the main relation involved in the competency question.

- Modifier, which is employed to impose restrictions on some entities or values.

- Domain-independent Element, which is an element that can occur across different knowledge domains. Some most commonly used domain-independent elements are temporal and spatial elements.

Based on these features, this work categorises competency questions into archetypes, which are shown in Table $2.1^{5}$

To be able to determine whether a competency question can be answered, the authors employ the notion of presupposition from linguistics (Beaver, 1997) to capture the ontology requirements implied by them. The term presupposition is used to refer to a special condition that must be met for a linguistic expression to have a denotation (Beaver, 1997). Therefore, this work analyses the presuppositions implied by each feature listed above and, therefore, by each archetype. For example, regarding the selection questions, the competency question "Which pizzas contain pork?" implies that pork is allowed to be contained in pizzas, i.e. Pizza $\sqcap \exists$ contains.Pork should be satisfiable. Otherwise, no pizza can contain pork at all.

\footnotetext{
${ }^{5}$ In the table, $\mathrm{CE}=$ class expression, $\mathrm{OPE}=$ object property expression, $\mathrm{DP}=$ datatype property, $\mathrm{I}=$ individual, $\mathrm{NM}=$ numeric modifier, $\mathrm{PE}=$ property expression, $\mathrm{QM}=$ quantity modifier.
} 
Table 2.1: Competency question archetypes (Ren et al. 2014)

\begin{tabular}{|l|l|l|}
\hline ID & Pattern & Example \\
\hline 1 & Which [CE1] [OPE] [CE2]? & Which pizzas contain pork? \\
\hline 2 & How much does [CE] [DP]? & How much does Margherita Pizza weigh? \\
\hline 3 & What type of [CE] is [I]? & $\begin{array}{l}\text { What type of software (API, Desktop ap- } \\
\text { plication etc.) is it? }\end{array}$ \\
\hline 4 & Is the [CE1] [CE2]? & Is the software open source development? \\
\hline 5 & What [CE] has the [NM] [DP]? & What pizza has the lowest price? \\
\hline 6 & What is the [NM] [CE1] to [OPE] [CE2]? & $\begin{array}{l}\text { What is the best/fastest/most robust soft- } \\
\text { ware to read/edit this data? }\end{array}$ \\
\hline 7 & Where do I [OPE] [CE]? & Where do I get updates? \\
\hline 8 & Which are [CE]? & Which are gluten free bases? \\
\hline 9 & When did/was [CE] [PE]? & When was the 1.0 version released? \\
\hline 10 & What [CE1] do I need to [OPE][CE2]? & $\begin{array}{l}\text { What hardware do I need to run this soft- } \\
\text { ware? }\end{array}$ \\
\hline 11 & Which [CE1] [OPE] [QM] [CE2]? & Which pizza has the most toppings? \\
\hline 12 & Do [CE1] have [QM] values of [DP]? & Do pizzas have different values of size? \\
\hline
\end{tabular}

Finally, using these presuppositions associated with each archetype, the authors propose to formalise each question into a SPARQL query in order to verify that the associated competency question is satisfied by the ontology. They claim that such queries could be generated automatically based on the structure of the requirement; however, the method to generate such queries is out of scope of the work.

\subsubsection{Wiśniewski and colleagues' requirement analysis}

The work proposed by Wiśniewski and colleagues (Wiśniewski et al., 2019) focuses on the association between linguistic patterns and SPARQL-OWL queries (Kollia et al. 2011). The authors analysed 234 requirements written as competency questions associated with 5 different ontologies and extracted 81 distinctive linguistic patterns. This set of linguistic patterns extended the one proposed by Ren and colleagues, which was based on a smaller dataset of requirements written as competency questions.

To perform the analysis, the authors proposed a method that includes the following steps:

1. Chunks and pattern candidates. In order to identify regularities among collected questions, the authors studied the linguistic structure of every requirement in the 
dataset. Because the dataset of requirements does not contain any pair of questions consisting of identical sequences of words, they proposed a pattern detection procedure to identify more general groups.

2. Pattern candidate filtering. Since a pattern should be something that is regularly repeated, a pattern candidate in order to be accepted as a pattern should be observed more than once in a dataset. Therefore, the authors identified placeholders in the candidate patterns. A placeholder is a fragment of text that should be filled with some class or instance from the ontology. It is introduced to indicate that the requirement makes sense for multiple placeholder fillings and each possible filling may be used to produce a requirement. As an example, the requirement Does [this softwarel provide XML editing? includes the placeholder /this software], which can be replaced with some classes defined in the ontology.

3. Pattern semantics. The previous steps produced some patterns that are semantically the same but that differ in minor aspects such as using plural and singular verbs, using synonyms or using words that could be removed from the requirement without changing its meaning. The authors joined semantically the same patterns and unify all cases, e.g., Is there EC1 for EC2? and Are there any EC1 for EC2? represent the same pattern.

In this work, the authors concluded that there can be multiple SPARQL-OWL queries for one distinct linguistic pattern, which is due to the different ways an ontology engineer can represent knowledge in the ontology. Moreover, there can be multiple linguistic patterns for a single SPARQL-OWL query, because there are different ways to formulate the same thing in natural language.

As an example of association, the following SPARQL-OWL query was proposed for the linguistic patterns "What types of EC1 are EC2?" and "What EC1 is of EC2 with respect to EC3?". In these patterns, the term EC refers to an entity chunk, i.e., a fragment of text describing an object that is likely to be represented in the ontology:

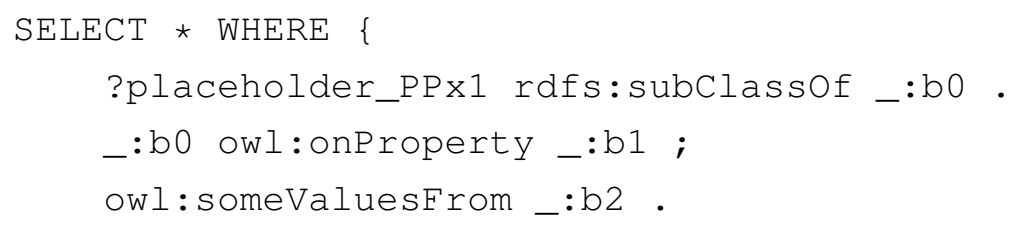


?placeholder_PPx1 rdfs:subclassof _:b3

\}

\subsubsection{Ontology verification testing methods}

Apart from global methodological approaches with a focus on ontology testing, several testing approaches which defend the importance of verifying ontologies through their ontology requirements have been developed to the date. Each of these approaches focuses on some testing aspect: methodological background, test implementation, or traceability between the ontology and the tests. The following sections describe the main approaches for ontology testing.

These testing approaches propose different types of tests, such as tests oriented to SPARQL queries which work over classes, properties and instances in the ontology, to inference analysis and to error provocation. In all of these tests it is needed to compare whether the output of the test is the same as the expected output of the competency question in order to know if the test is passed or not.

\subsubsection{Blomqvist and colleagues' testing approach}

Blomqvist and colleagues (Blomqvist et al., 2012) present an agile approach which includes a methodological background for testing and introduces three main types of tests, namely, (1) competency question verification, (2) inference verification, and (3) error provocation. The first type of test is oriented to the reformulation of the competency questions as SPARQL queries after adding test data related to the query to be reformulated, i.e., it does check classes and properties in the ontology. The second type verifies that the inference mechanisms are in place. Finally, the third one is intended to expose faults.

In order to keep tests separated from the ontology to be tested, this proposal represents a test case as an OWL ontology, which includes properties for describing each test case. The main concepts of the metamodel ontology and their relations are illustrated in Figure 2.9.

Although this methodology proposes types of tests to verify requirements, it does not describe the relation between such requirements and the tests, e.g., when to use each type of test. Moreover, the analysis of the requirements is out of scope of this methodology. 




Figure 2.9: Main concepts of the TestCase metamodel Blomqvist et al. 2012

\subsubsection{CQChecker}

CQChecker ${ }^{6}$ (Bezerra et al. 2014$)$ is a tool proposed by Bezerra and colleagues which provides a mechanism that allows verifying whether the ontology meets its corresponding competency questions by supporting both assertional and terminological queries. To accomplish that, the authors distinguish several types of competency question, such as competency questions which work over classes and their relations or competency question which work over instances. They create a modular architecture where each module treats each different competency question type. Figure 2.10 depicts the architecture of CQChecker.

The authors identify three types of competency questions based on how they are specified. These three types of competency questions are a subset of the ones provided by Ren and colleagues described in Section 2.3.3.

1. Competency questions which work over classes and their relations.

2. Decision problems expressed as competency questions. In this type, the answer permitted to the question can only be true or false.

3. Competency questions are expressed in an interrogative form which works only over instances.

\footnotetext{
${ }^{6}$ CQChecker is available in the following URL: https://sourceforge.net/projects/ cqchecker. The last update was on December 5, 2016.
} 


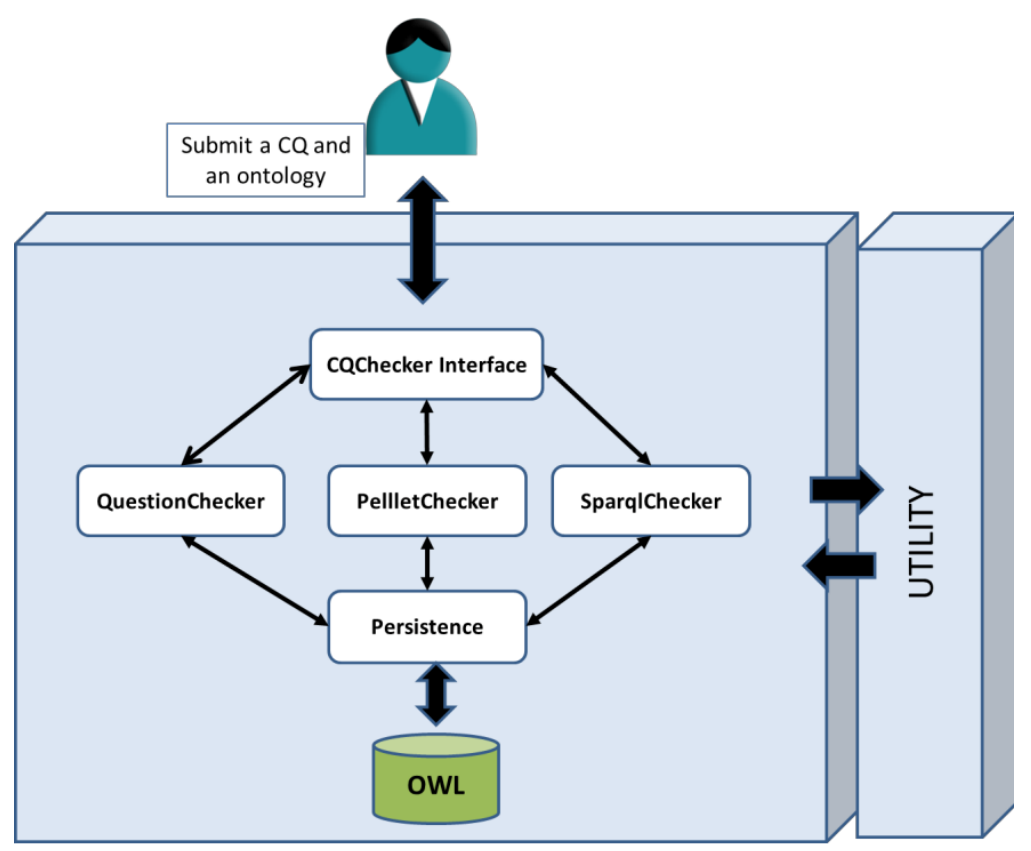

Figure 2.10: Architecture of CQChecker (Bezerra et al., 2014)

To use the tool, first users have to submit a competency question and an ontology, and then the competency question will be analysed and directed to one of the three modules, i.e., QuestionChecker (competency question of type 1), PelletChecker (competency question of type 2) or SparqlChecker (competency question of type 3).

The tool analyses the competency question in order to classify it into one of the three modules, according to the possible answer it is supposed to retrieve. Then, the system directs the competency question to the corresponding module, where it will be converted and checked.

\subsubsection{Ontology testing methods for query execution}

While in previous sections several testing approaches and tools based on competency questions have been reviewed, this section focuses on tools that query an ontology and that can be used for ontology verification.

These approaches are not oriented to the definition of types of tests based on the different types of requirements, but to the definition of different types of queries that can be executed on an ontology. Although these approaches are not directly oriented 
to the ontology verification activity, they can be useful for analysing how they support the execution of queries to test an ontology.

\subsubsection{OntologyTest}

OntologyTest 7 (García-Ramos et al. 2009) is a Java application that allows the elaboration and execution of tests to evaluate OWL DL ontologies. Each test comprises an optional set of instances, a query and the expected result. OntologyTest supports the following types of tests:

- Instantiation test. It specifies whether or not an individual belongs to a given class. An example of query could be Paracetamol(paracetamol102), which checks whether the instance paracetamol102 is of class Paracetamol.

- Recovering test. It allows the user to specify a list with all instances that must belong to a particular class. An example of query could be Paracetamol, and an example of expected result could be /paracetamol101, paracetamol102 , paracetamol103], which represent all the instances of the class Paracetamol.

- Realisation test. It specifies the most specific class that must be instantiated by an individual. For instance, the query paracetamol101 and the expected result Paracetamol, check whether paracetamol101 is an instance of the class Paracetamol.

- Satisfaction test. It specifies whether an inconsistency should occur in the ontology after adding a new instance of a class. An example of query could be Paracetamol(paracetamol102), to check whether the addition of the instance paracetamol102 leads to an inconsistency.

- Classification test. It specifies a list with all classes that an individual must belong to. For instance, the query paracetamol101 with the expected result /paracetamol, chemicalSubstance, thing] indicates all the classes to which the instance paracetamol101 belongs to.

- SPARQL test. The query is written in SPARQL, and the results are associated with the variables of the query.

\footnotetext{
${ }^{7}$ OntologyTest is not available online.
} 


\subsubsection{VoCol}

VoCo 8 Halilaj et al. 2016) is an integrated environment that supports the development of vocabularies using version control systems. VoCol supports a round-trip model of vocabulary development, consisting of three core activities, i.e., modelling, population, and testing. For testing, VoCol allows formulating SPARQL queries which represent competency questions.

Figure 2.11 illustrates the architecture and workflow of VoCol. In order to implement VoCol as an integrated environment, it follows the principles of Component Based Software Development (CBSD) (Heineman and Councill, 2001), which promotes the reuse of components to develop large-scale systems. Therefore, VoCol is composed by a set of smaller components according to the functionalities they provide. Among these components, VoCol includes a querying service which integrates a SPARQL endpoint synchronised with the latest version of the ontology, which could be used to check whether the ontology fulfils the requirements.

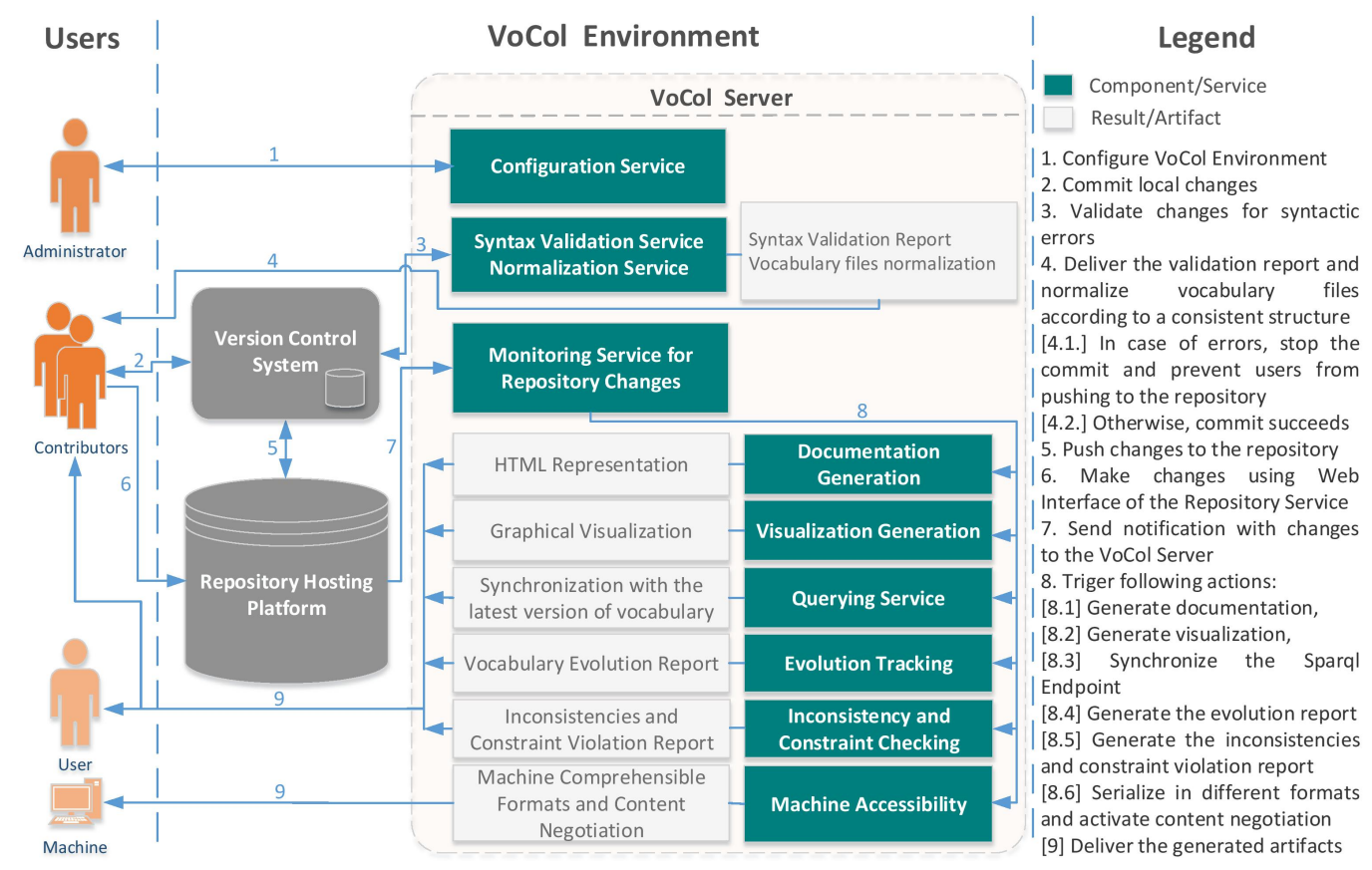

Figure 2.11: VoCol architecture and workflow (Halilaj et al. 2016)

\footnotetext{
${ }^{8} \mathrm{VoCol}$ is available in the following URL: https://vocol.iais.fraunhofer.de/. The last update was on March 3, 2020.
} 
Although VoCol provides a service that allows users to execute SPARQL queries on an ontology, how to create such queries is out of scope of the tool.

\subsubsection{Ontology testing integrated into ontology development method- ologies}

Keet and Ławrynowicz proposed a test-driven development (TDD) of ontologies (Keet and Lawrynowicz, 2016), which is an ontology development approach inspired by testdriven development in Software Engineering (Beck, 2003). This TDD, which is based on the idea of writing a failing test before writing any code, ensures that what is added to the program core does indeed have the intended effect specified upfront. Moreover, the test-driven development methodology principle increases the understanding of the ontology authoring process and the logical consequences of an axiom.

The steps to be performed within the TDD testing approach, which is depicted in Figure 2.12 along with an ontology life-cycle that focuses on TDD, are summarised as follows:

1. To check whether the vocabulary elements of $x$ are in ontology $O$ (itself a TDD test).

2. To run TDD test twice:

(a) To run the first execution of the tests. This first execution should fail.

(b) To update the ontology (add $x$ ).

(c) To run the test again which should pass, checking that there is no new inconsistency or undesirable deduction.

3. To run all previous successful tests, which should pass. This step represents the regression testing.

This approach is oriented to the definition of tests and their execution on an ontology. Figure 2.12 shows that a potential input to write such tests is a set of competency questions (CQ), i.e., the ontology requirements; however, how to write such tests from requirements is out of scope of the methodology. 


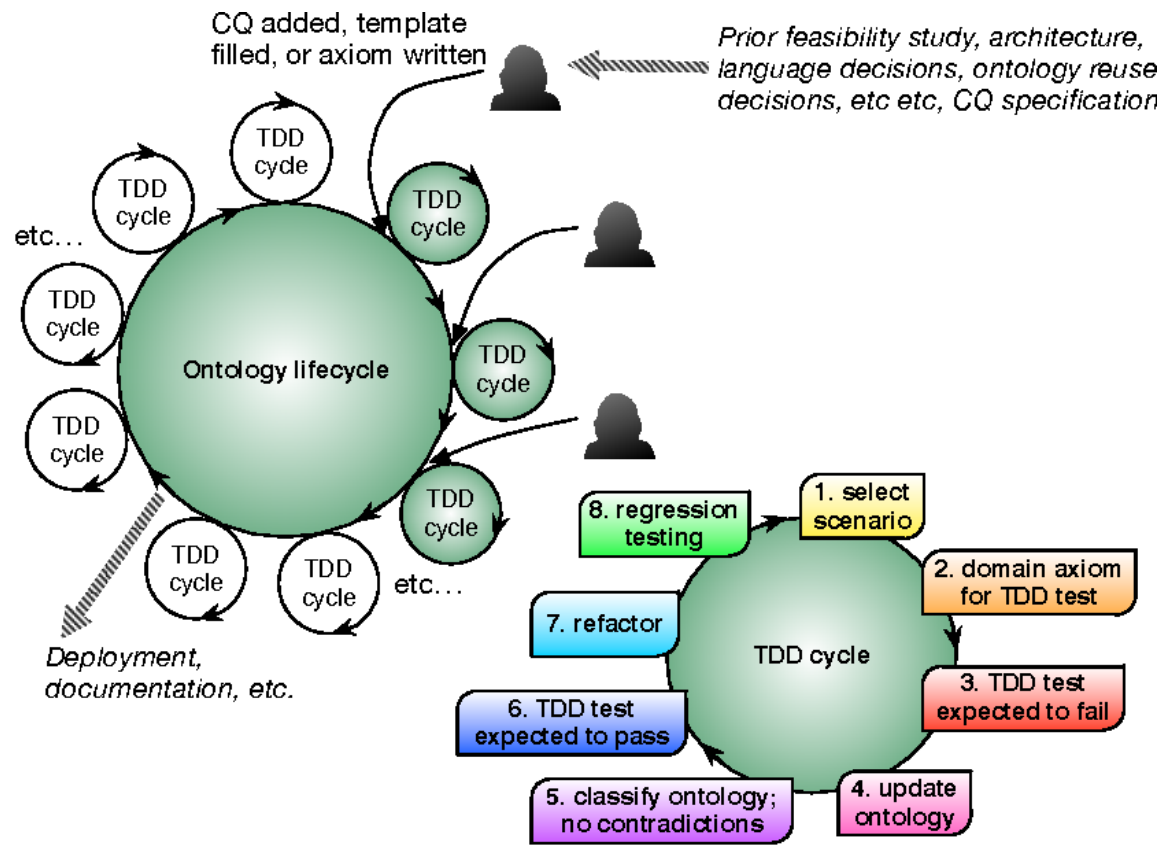

Figure 2.12: Summary of the possible ontology lifecycle that focuses on TDD, and the sequence of steps of the TDD procedure (Keet and Ławrynowicz, 2016)

This TDD methodology is supported by the TDDOnto tool, which has been first introduced by Ławrynowicz and Keet (Ławrynowicz and Keet, 2016) and further developed as TDDOnto2 $2^{9}$ (Davies et al. 2017). This tool checks whether a particular axiom is present in an ontology. TDDOnto2 relies on a logic-based model of TDD unit testing and on generalised versions of the algorithms of TDD (Keet and Ławrynowicz, 2016) to support the tests related to any OWL 2 class expression.

TDDOnto2 uses the OWL AP 10 and it is openly available as a plugin for Protégé 11 The workflow for an actual test in TDDonto2 is the following:

1. To type an axiom in the test text box.

2. To evaluate it immediately or to add it to the test suite to evaluate it later.

3. To get the result, which can be one of the following:

\footnotetext{
${ }^{9}$ The last version of TDDOnto2 is available in the following URL: https://github.com/ kierendavies/tddonto2. The last update was on August 23, 2018.

${ }^{10} \mathrm{http}$ // owlcs.github.io/owlapi/

${ }^{11}$ https://github.com/kierendavies/tddonto2
} 
- The axiom is already entailed.

- Adding the axiom would result in a contradiction or unsatisfiable class.

- The axiom is absent and will not lead to a contradiction if added.

4. To remove the axiom under test or to add it to the ontology.

In the previous testing approaches the results of the tests execution must be analysed to conclude whether they are passed or not. However, in the TDD approach this analysis is not required since the obtained result indicates whether the tests are passed.

\subsubsection{Ontology metrics to support the ontology life-cycle}

In the Ontology Engineering field, metrics have also been an important research topic due to the fact that they can help to assess and to qualify ontologies or to detect ontology problems. Different proposals for ontology metrics have been introduced in the past years. However, they are focused on measuring aspects of the ontology implementation, leaving aside other artefacts related to the ontologies, such as the ontology specification or the tests generated during the testing process. This absence of metrics hinders the monitoring of the ontology development process during the ontology life-cycle. Metrics related to testing results, as well as to requirements specification, can help to control and be aware of the status of the ontology and the development process.

The work presented by Duque-Ramos and colleagues (Duque-Ramos et al., 2013) already creates a classification of ontology metrics; however, their proposed classification is based on the use that is made of the metrics, e.g., functional adequacy, compatibility or maintenance, among others. Instead, the metrics presented in this section are classified according to the product from which the metrics are extracted. Due to the fact that all the metrics in the literature are extracted from the ontology implementation, the classification presented in the following sections is based on the different ontology implementation elements needed to calculate them, e.g., ontology classes, ontology hierarchies, ontology properties and ontology axioms.

\subsubsection{Metrics related to ontology classes}

Yao and colleagues (Yao et al. 2005) propose a set of ontology cohesion metrics to measure the modular relatedness of OWL ontologies. Cohesion traditionally refers to 
the degree to which the elements in a module belong together. Regarding ontology classes, the metrics they propose include Number of root classes (NoR), which counts the number of classes that have no superclass and Number of leaf classes (NoL), which counts the number of classes that have no subclass.

Zhe and colleagues (Zhe et al. 2006) are focused on the problem that ontology complexity has to remain tractable during evolution. Therefore, they analyse the concepts, together with the hierarchies in a conceptual model, which reflect the fundamental complexity. The proposed test suite includes the Total number of concepts or classes (TNOC) metric.

Another set of ontology class metrics is the one presented by Kang and colleagues (Kang et al., 2012), whose metrics are used to predict reasoning performance. Regarding ontology, the set of metrics includes the Number of children (NOC), and Expression richness ( $\mathrm{RCH})$ metrics. The first metric measures the number of immediate subclasses subordinated to a class in the class hierarchy while the latter one measures the ratio between the number of anonymous class expressions and the total number of class expressions. This work also proposes Depth of inheritance (DIT), which measures the depth of inheritance of a class, and Cyclomatic complexity (CYC), which measures the number of linearly independent paths in the ontology graph.

Orme and colleagues (Orme et al., 2006) propose two different metrics to measure the level of coupling in ontologies using ontology classes, namely, Number of external classes (NEC), which refers to the number of distinct external classes used to define new classes and properties in the ontology, and Number of references to external classes $(R E C)$.

Gangemi and colleagues (Gangemi et al. 2006) define a set of metrics related to the ontology structure which includes Absolute leaf cardinality, which measures the number of leaf nodes in an ontology and is similar to Number of leaf classes (NoL) already presented by Yao and colleagues (Yao et al., 2005).

There are also tools, such as the ontology editors Protég $\AA^{12}$ and WebProtég $€^{13}$ which calculate ontology metrics. These editors calculate, among other metrics, Number of classes and Max siblings, which count the maximum number of siblings for any class.

$1 2 \longdiv { \text { https: //protege.stanford.edu/ } }$

13 https://webprotege.stanford.edu/ 
The tool OntoMetrics (Lantow, 2016) also provides, besides the metrics already defined in Gangemi and colleagues' work (Gangemi et al., 2006), a new metric related to ontology classes named Equivalence ratio.

The OQuaRE framework (Duque-Ramos et al., 2011), which adapts the SQuaRE standard (ISO, 2011a) related to software quality evaluation for evaluating ontologies, also includes metrics related to ontology classes. These metrics include Lack of Cohesion in Methods, which measures the relatedness of classes, and Redundancy, which measures the capability of the ontology to be informative. This latter metric can also be related to the redundancy of properties and instances in an ontology.

\subsubsection{Metrics related to the class hierarchy}

Regarding metrics related to the class hierarchy, Yao and colleagues (Yao et al., 2005) propose Average depth of inheritance of all leaf nodes (ADIT-LN), which is defined as the sum of depths of all paths divided by the total number of paths.

Gangemi and colleagues (Gangemi et al. 2006) also propose a set of metrics related to the hierarchy to measure the ontology structure. Some of the ontology structure metrics proposed by these authors are Tangledness, which is related to the multi-hierarchical nodes of a graph; Absolute depth, which is related to the cardinality of paths in a graph; or Absolute breadth, which is related to the levels in a graph.

Zhe and colleagues (Zhe et al., 2006) also propose a set of tests related to hierarchies in order to analyse ontology complexity. The proposed test suite includes the Total number of paths (TNOP) and Average paths per concept, which is calculated by dividing TNOP by TNOC.

Kang and colleagues (Kang et al. 2012) also propose metrics related to hierarchies for analysing reasoning performance, which are Depth of inheritance (DIT) and Cyclomatic complexity $(C Y C)$. This latter metric measures the number of linearly independent paths in the ontology graph.

Finally, the OQuaRE framework (Duque-Ramos et al., 2011) proposes the Depth of subsumption hierarchy, which measures the length of the largest path from Thing to a leaf class. 


\subsubsection{Metrics related to ontology properties}

With relation to metrics extracted from the ontology properties, Zhe and colleagues (Zhe et al. 2006) propose Total number of relations (TNOR) and Average relations per concept, which is calculated by dividing TNOR by TNOC.

Tartir and colleagues (Tartir et al., 2005) provide a set of metrics which evaluates ontology design and its potential for rich knowledge representation from the ontology properties, such as Relationship Richness and Attribute Richness. The first metric reflects the diversity and placement of relations in the ontology, while the second one measures the number of attributes for each class.

The tool OntoMetrics (Lantow, 2016) also calculates metrics related to ontology properties for analysing the design of the ontology, such as Class/Relation ratio, which describes the ratio between the classes and the relations in the ontology, and Inverse relations ratio, which describes the ratio between the inverse relations and all relations.

\subsubsection{Metrics related to ontology individuals}

Tartir and colleagues (Tartir et al. 2005) provide a set of metrics which evaluates the placement of instance data within the ontology and the effective usage of the ontology to represent the knowledge modelled in the ontology. This set includes Class Richness, which is related to how instances are distributed across classes and Average Population, which is defined as the number of instances divided by the number of classes. Moreover, it also includes Cohesion, which is calculated as the number of separate connected components in instances.

Finally, OntoMetrics ( $\overline{\text { Lantow }}$ 2016) provides new metrics related to individuals, such as Class Importance, which is the percentage of instances that belong to classes at the inheritance subtree rooted at the current class with respect to the total number of instances, or Class Fullness, which measures the expected number of instances of each class.

\subsubsection{Metrics related to ontology axioms}

Ma and colleagues (Ma et al., 2010) propose a set of metrics to measure the cohesion of ontologies. They propose Number of ontology partitions (NOP), which measures the number of semantic partitions of a knowledge base, this is, the number of parts 
in an ontology that are semantically unrelated to each other, Number of minimally inconsistent subsets (NMIS), which measures the minimally inconsistent subsets in a knowledge base, and Average value of axiom inconsistencies (AVAI).

The tool Protégé also provides metrics related to axioms, such as the Number of Axioms.

\subsubsection{Metrics related to ontology metadata}

Regarding metadata metrics, Tartir and colleagues (Tartir et al., 2005) propose Readability, which indicates the existence of human-readable descriptions in the ontology such as comments or labels.

Orme and colleagues (Orme et al., 2006) propose the Number of referenced includes (RI) metric, which represents the number of includes in the ontology implementation.

Additionally, the tool OntoCheck (Schober et al. 2012) displays the percentage and the absolute number of nodes having exactly, at least or at most a certain number of selectable metadata elements, parents and children, direct superclasses, subclasses, and class usages.

\subsection{Conclusions}

This chapter has introduced the state of the art related to verification in the Software Engineering and the Ontology Engineering field. Therefore, some of the most relevant contributions for ontology verification in the Software Engineering and Ontology Engineering fields are described.

After analysing the state of the art in Software Engineering and in Ontology Engineering, it can be stated that the Ontology Engineering field has been inspired by the Software Engineering field, adapting software methodologies and techniques for ontologies, e.g., unit testing or TDD.

Moreover, it was found that the degree of maturity of the Ontology Engineering field with regards to verification is low when compared to the Software Engineering field. In this latter field, verification testing plays an important role in software development, with well-known testing techniques and methodologies that are used by the community, such as TDD or BDD. Moreover, both verification testing and metrics are 
integrated into the software development process. However, in the Ontology Engineering field ontology verification has been neglected and, although ontology development methodologies mention the need for requirements, only a few testing approaches deal with this issue.

Among the ontology development methodologies that consider ontology verification, some limitations have been identified. It is true that they agree on the importance of ontology requirements, since these requirements identify the purpose and the needs that should be included in an ontology. Some of the approaches explicitly specify that a good way to verify such requirements is the generation of SPARQL queries to check whether requirements are satisfied, such as in the SAMOD methodology or in the eXtreme Design one. However, in these ontology development methodologies the verification activity consists of high level steps and there is a lack of guidelines to determine how to generate such queries.

Additionally, in the testing approaches and tools there is a lack of guidelines to help developers and practitioners to create the needed tests from such requirements.

Moreover, the majority of these testing approaches do not consider traceability between requirements, tests and ontologies, and are oriented to ontology engineers. Therefore, they pay little attention to how both domain experts and users should also become part of the ontology verification activity.

Tables 2.2 and 2.3 summarise the conclusions obtained from the analysis of the testing approaches in Ontology Engineering, indicating how methods and tools support traceability, the definition, implementation and execution of tests, and which is the targeted audience for each one.

Along this section, several approaches for conformance testing developed by standardisation bodies have been detailed. These approaches are oriented to software engineering and industrial products with the aim of ensuring quality by analysing whether such products satisfied the requirements of a set of standards. However, in the Ontology Engineering field there is a lack of works on analysing conformance. Until now, the analysis of conformance in Ontology Engineering has been only focused on evaluating the conformance between semantic technologies and the ontology representation languages, e.g., OWL conformance (García-Castro, 2009) or SPARQL conformance ${ }^{14}$

\footnotetext{
${ }^{14}$ https://www.w3.org/TR/rdf-sparql-query/\#conformance
} 
Table 2.2: Summary of conclusions regarding ontology testing within ontology development methodologies

\begin{tabular}{|c|c|c|c|c|c|}
\hline & $\begin{array}{l}\text { Grüninger } \\
\text { and Fox }\end{array}$ & On-To-Knowledge & $\begin{array}{l}\text { EXtreme } \\
\text { ontology }\end{array}$ & $\begin{array}{l}\text { eXtreme } \\
\text { Design }\end{array}$ & SAMOD \\
\hline \multicolumn{6}{|c|}{ Dimension } \\
\hline Traceability & Not treated & Not treated & Not treated & Not treated & Not treated \\
\hline \multicolumn{6}{|c|}{ Detailed Guidelines for Processes and Activities } \\
\hline $\begin{array}{l}\text { Requirement } \\
\text { specification }\end{array}$ & $\begin{array}{l}\text { Competency } \\
\text { questions }\end{array}$ & $\begin{array}{l}\text { Competency } \\
\text { questions }\end{array}$ & $\begin{array}{l}\text { Competency } \\
\text { questions }\end{array}$ & $\begin{array}{l}\text { Competency } \\
\text { questions }\end{array}$ & $\begin{array}{l}\text { Competency } \\
\text { questions }\end{array}$ \\
\hline Definition of tests & Not provided & Not provided & Not provided & Not provided & Not provided \\
\hline $\begin{array}{l}\text { Implementation } \\
\text { and execution of } \\
\text { tests }\end{array}$ & Not provided & Not provided & Not provided & $\begin{array}{l}\text { SPARQL } \\
\text { queries }\end{array}$ & $\begin{array}{l}\text { SPARQL } \\
\text { queries }\end{array}$ \\
\hline \multicolumn{6}{|c|}{ Audience } \\
\hline Targeted audience & $\begin{array}{l}\text { Ontology } \\
\text { engineers }\end{array}$ & $\begin{array}{l}\text { Ontology } \\
\text { engineers }\end{array}$ & $\begin{array}{l}\text { Ontology } \\
\text { engineers }\end{array}$ & $\begin{array}{l}\text { Ontology } \\
\text { engineers }\end{array}$ & $\begin{array}{l}\text { Ontology } \\
\text { engineers }\end{array}$ \\
\hline \multicolumn{6}{|c|}{ Technological support } \\
\hline Tool & No & No & No & No & No \\
\hline
\end{tabular}

Table 2.3: Summary of conclusions regarding ontology testing approaches

\begin{tabular}{|c|c|c|c|c|c|c|c|}
\hline & $\begin{array}{l}\text { Ren and } \\
\text { colleagues }\end{array}$ & $\begin{array}{l}\text { Wiśniewski } \\
\text { and colleagues }\end{array}$ & $\begin{array}{l}\text { Blomqvist } \\
\text { and } \\
\text { colleagues }\end{array}$ & CQChecker & OntologyTest & VoCol & TDD \\
\hline \multicolumn{8}{|c|}{ Dimension } \\
\hline Traceability & Not treated & Not treated & $\begin{array}{l}\text { Test stored in } \\
\text { an ontology }\end{array}$ & Not treated & Not treated & Not treated & Not treated \\
\hline \multicolumn{8}{|c|}{ Detailed Guidelines for Processes and Activities } \\
\hline $\begin{array}{l}\text { Requirement } \\
\text { specification }\end{array}$ & $\begin{array}{l}\text { Patterns of } \\
\text { competency } \\
\text { questions }\end{array}$ & $\begin{array}{l}\text { Patterns of } \\
\text { competency } \\
\text { questions }\end{array}$ & $\begin{array}{l}\text { Comptency } \\
\text { questions }\end{array}$ & $\begin{array}{l}\text { Comptency } \\
\text { questions }\end{array}$ & Not provided & $\begin{array}{l}\text { Not provid } \\
\text { ed }\end{array}$ & $\begin{array}{l}\text { Not provid } \\
\text { ed }\end{array}$ \\
\hline $\begin{array}{l}\text { Definition of } \\
\text { tests }\end{array}$ & $\begin{array}{l}\text { List of types } \\
\text { of competency } \\
\text { questions }\end{array}$ & $\begin{array}{l}\text { List of types } \\
\text { of competency } \\
\text { questions }\end{array}$ & $\begin{array}{l}\text { List of } \\
\text { types of tests }\end{array}$ & $\begin{array}{l}\text { Not provid- } \\
\text { ed }\end{array}$ & $\begin{array}{l}\text { Not provid- } \\
\text { ed }\end{array}$ & $\begin{array}{l}\text { Not provid- } \\
\text { ed }\end{array}$ & $\begin{array}{l}\text { Tests as } \\
\text { OWL } \\
\text { axioms }\end{array}$ \\
\hline $\begin{array}{l}\text { Implementa- } \\
\text { tion and } \\
\text { execution of } \\
\text { tests }\end{array}$ & $\begin{array}{l}\text { SPARQL } \\
\text { queries and } \\
\text { Inference } \\
\text { checking }\end{array}$ & $\begin{array}{l}\text { SPARQL } \\
\text { queries }\end{array}$ & $\begin{array}{l}\text { SPARQL } \\
\text { queries }\end{array}$ & $\begin{array}{l}\text { SPARQL } \\
\text { queries }\end{array}$ & $\begin{array}{l}\text { SPARQL } \\
\text { queries }\end{array}$ & $\begin{array}{l}\text { SPARQL } \\
\text { queries }\end{array}$ & $\begin{array}{l}\text { Presence or } \\
\text { absence of } \\
\text { axioms }\end{array}$ \\
\hline \multicolumn{8}{|c|}{ Audience } \\
\hline $\begin{array}{l}\text { Targeted } \\
\text { audience }\end{array}$ & $\begin{array}{l}\text { Ontology } \\
\text { engineers }\end{array}$ & $\begin{array}{l}\text { Ontology } \\
\text { engineers }\end{array}$ & $\begin{array}{l}\text { Ontology } \\
\text { engineers }\end{array}$ & $\begin{array}{l}\text { Ontology } \\
\text { engineers }\end{array}$ & $\begin{array}{l}\text { Ontology } \\
\text { engineers }\end{array}$ & $\begin{array}{l}\text { Ontology } \\
\text { engineers }\end{array}$ & $\begin{array}{l}\text { Ontology } \\
\text { engineers }\end{array}$ \\
\hline \multicolumn{8}{|c|}{ Technological support } \\
\hline Tool & No & No & Yes & Yes & Yes & Yes & Yes \\
\hline
\end{tabular}

rather than on testing whether an ontology is compliant with the specification of a standard. Table 2.4 summarises the main characteristics of the conformance approaches presented in this section.

Table 2.4 shows that while the ISO/IEC 9464 and the ETSI approaches are focused on describing how to write and reuse tests, e.g., developing a particular testing language, 
Table 2.4: Main characteristics of the conformance testing approaches

\begin{tabular}{|c|c|c|c|}
\hline & & & \\
\hline & ISO/IEC & ETSI & W3C \\
\hline \multicolumn{4}{|c|}{ Detailed Guidelines for Activities } \\
\hline Activities definition & $\begin{array}{l}\text { Definition, im- } \\
\text { plementation and } \\
\text { execution of tests }\end{array}$ & $\begin{array}{l}\text { Definition, im- } \\
\text { plementation and } \\
\text { execution of tests }\end{array}$ & Not treated \\
\hline Language definition & TTCN & TTCN-3 & Not treated \\
\hline \multicolumn{4}{|c|}{ Publication } \\
\hline Reusability support & Abstract tests & Abstract tests & $\begin{array}{l}\text { Depends on the stan- } \\
\text { dard }\end{array}$ \\
\hline $\begin{array}{l}\text { Publication of conformance } \\
\text { clauses }\end{array}$ & Not treated & Not treated & $\begin{array}{l}\text { Requirements and } \\
\text { good practices for } \\
\text { conformance clauses } \\
\text { publication }\end{array}$ \\
\hline
\end{tabular}

the $\mathrm{W} 3 \mathrm{C}$ is focused on providing guidelines on how to specify conformance clauses.

Finally, regarding the ontology engineering metrics, even if there is a large amount of work in related to metrics, the metrics found in the literature only analyse the ontology implementation, leaving aside the other artefacts obtained during the ontology development process, such as the requirements specification or the tests. A detailed analysis of the metrics also shows that the metrics are mainly focused on the ontology structure, especially on ontology classes. Therefore, there is a lack of metrics related to the artefacts generated and used during the testing process. Metrics should provide information about the status of the ontology during its life-cycle, which helps planning and managing the ontology development process in an efficient way.

Table 2.5 summarises the ontology metrics described in this chapter according to the artefact from which they are extracted. The table shows that, even if there is a large amount of work in the Ontology Engineering field related to metrics, the metrics found in the literature only analyse the ontology implementation when making measurements, leaving aside the other artefacts obtained during the ontology development process, such as the documentation or the requirements specification. A detailed analysis on the metrics also shows that, even if the authors relate the metrics to different aspects, such as coupling or cohesion, they are mainly focused on the ontology structure, especially on the ontology classes. 
Table 2.5: Summary of metrics in Ontology Engineering

\begin{tabular}{|c|c|c|c|c|}
\hline & Ontology implementation & $\begin{array}{c}\text { Requirements } \\
\text { specification }\end{array}$ & Tests & $\begin{array}{c}\text { User } \\
\text { documentation }\end{array}$ \\
\hline \multirow{5}{*}{ Metrics } & \multicolumn{4}{|c|}{ Product category } \\
\hline & $\begin{array}{l}\text { Class metrics (e.g., TNOC, NoR, NoL ) } \\
\text { Hierarchy metrics(e.g., ADIT-LN, Absolute depth) } \\
\text { Property metrics ( e.g., TNOR, Relationship richness) } \\
\text { Individual metrics (e.g., Class Richness, Class Fullness) } \\
\text { Axiom properties metrics (e.g., NOP, AVAI ) } \\
\text { Metadata metrics (e.g., RI, Readability, OntoCheck } \\
\text { metrics) }\end{array}$ & - & - & - \\
\hline & \multicolumn{4}{|c|}{ Lifecycle (metrics not related to any artefact) } \\
\hline & \multicolumn{4}{|c|}{ Process category } \\
\hline & \multicolumn{4}{|l|}{-} \\
\hline
\end{tabular}





\section{Chapter 3}

\section{Work Objectives}

This chapter presents the main objectives of this thesis and the open research problems it aims to address (Section 3.1) together with its main contributions (Section 3.2. Moreover, the chapter also introduces the thesis assumptions, hypotheses and restrictions (Section 3.3), as well as the plan for the evaluation of the stated hypotheses (Section 3.4). The chapter concludes with the research methodology and research process followed during the development of this thesis (Section 3.5.)

\subsection{Thesis objectives}

The overall objective of this thesis is to advance the current state of the art in the ontology verification field. In order to attain this goal, the following sub-objectives should also be pursued:

O1. To help ontology engineers and practitioners to verify their ontologies by specifying tests according to their ontological requirements.

O2. To extract information that supports during ontology development taking decisions and monitoring by means of metrics related to artefacts other than the ontology implementation, such as those generated and used during the ontology verification process.

O3. To automate the analysis of the degree of conformance between an ontology and a standard by executing tests according to the requirements of the standard. 
In order to achieve the first objective, from a methodological perspective the following open research problem must be solved:

- In the current testing methods for ontology verification no studies have been yet performed on how requirements are specified and translated into tests in order to guide developers and practitioners during the verification activity. Even more, the majority of these methods are oriented to ontology engineers (e.g., developing plugins for ontology engineers tools) but they pay little attention to how domain experts and users become part of the process. To this extent, there is a lack of testing methods that ease the ontology verification task for ontology engineers, domain experts and users.

From a technological perspective the following engineering problem must be solved:

- Current software implementations of testing methods are oriented to ontology engineers, e.g., focused on the generation and execution of SPARQL queries or oriented to Protégé plugins. Therefore, new technological support is needed to assist ontology engineers, domain experts and users during the ontology verification process.

In order to achieve the second objective, the following open research problem must be solved:

- In real-world projects it is necessary to be aware of the status of the development process, in order to be able to plan and manage it in an efficient way. In the Ontology Engineering field, metrics should provide information not only about the structure of the ontology but also about other artefacts, such as requirements or tests, which are generated during development processes. The information retrieved by metrics would allow ontology engineers and practitioners to analyse the ontology and its development process, as well as to make managerial evidencebased decisions. However, as presented in the analysis of the ontology metrics in the state of the art, there is a lack of metrics extracted from artefacts other than the ontology implementation, which hinders the extraction of information from the development process, such as from ontology verification activities. 
In order to achieve the third objective, from a methodological perspective the following open research problem must be solved:

- Standard ontologies are created to maximise semantic interoperability and to provide an agreed baseline approved by the community that can be used by other developers who want to describe a particular area of concern. Therefore, it is needed a method to ensure that an ontology conforms to some ontology standard, analysing the degree of overlaps and differences with it, with the goal of guaranteeing integrity and quality when describing a particular domain. However, there is a lack of methods for analysing ontology conformance with existing standards.

From a technological perspective the following engineering problem must be solved:

- In the Ontology Engineering field there is a lack of approaches for analysing ontology conformance and, consequently, there is a lack of tools to assist such approaches. Therefore, new technological support is needed to allow ontology engineers to analyse the conformance between ontologies and standards.

\subsection{Contributions}

This thesis aims at giving solutions to the open research problems identified in Section 3.1. Chapter 4 describes the solution proposed for the first objective (to help ontology engineers and practitioners to verify their ontologies), Chapter 5 describes the solution for the second objective (to provide metrics to allow evidence-based decision making) and Chapter 6 presents the solution for the third one (to automate the analysis of conformance between an ontology and a standard).

With regards to the first objective, this thesis supports the advance of the current state of the art with the following contributions:

C1. Method for ontology verification testing. This method proposes a set of activities within the testing process to systematise the design, implementation and execution of tests for verifying ontologies. Inspired by Software Engineering techniques, such as Keyword-driven testing, the use of a testing language based 
on keywords aims at facilitating the translation between requirements and tests, as well as the definition of tests for ontology practitioners. Therefore, this method uses a set of lexico-syntactic patterns to identify different types of requirements, which are used to define tests for ontology verification. Based on such lexicosyntactic patterns, a testing language was defined which uses keywords to identify the purpose of each test. The results of the testing activities are stored in a machine-readable format, allowing traceability between requirements, tests, and the ontology implementation.

With respect to the current state of the art, this contribution provides advancements in terms of a new testing language based on lexico-syntactic patterns that aim at helping ontology engineers and practitioners in the definition of tests.

C2. Themis, an online tool for supporting ontology testing. This software allows executing a set of tests, which follow the testing language defined in $C 1$, on one or more ontologies.

With respect to the current state of the art, this contribution provides advancement in terms of a web application for ontology verification oriented to ontology engineers, domain experts and users and that does not require any previous installation to be used.

The second objective of this thesis is to extract information that supports taking decisions using metrics. To this extent, this thesis presents new advances by providing the following contribution:

C3. A set of metrics related to the artefacts generated and used in the ontology verification process. These metrics are extracted from the artefacts generated and used in the testing method for ontology verification $(C 1)$, such as the implementation, the requirements and the tests, and are based on the needs of the different roles that are involved in the ontology development process, e.g., the stakeholders and the ontology engineers. The goal of these metrics is to provide information related to the ontology and its development process, such as which requirements and tests are satisfied, that can be used to analyse the status of an ontology and to take managerial decisions. 
With respect to the current state of the art, this contribution provides advancements in terms of a new set of ontology metrics that is related to the ontology development process. Such metrics go beyond the ontology implementation considering other artefacts such as those related to the ontology verification process.

Finally, concerning the third objective, this thesis presents new advances by providing the following contribution:

C4. Method for ontology conformance testing. This method for conformance testing is grounded on the method for ontology verification testing $(C 1)$, adapting it to the ontology conformance scenario where an ontology is verified against a given standard. This method uses several metrics (C3) that are related to requirements management and testing in order to analyse the degree of conformance between an ontology and the standard. Moreover, the Themis tool (C2) provides partial support to this conformance method.

With respect to the current state of the art, this contribution provides advancement in terms of an ontology conformance testing method that allows verifying whether an ontology satisfies the requirements of a standard. Such conformance testing method can also be applied to analyse the minimum common knowledge between a set of standards, with the aim of analysing how such standards cover their domain.

Contributions $C 1, C_{3}$ and $C_{4}$ are related to the methodological perspective, while contribution $C 2$ is related to the technological one.

\subsection{Work assumptions, hypotheses and restrictions}

This thesis was developed under a set of assumptions that help to explain the decisions taken for the achievement of the thesis goals; such assumptions are listed below:

A1. Ontological requirements are defined before implementing any ontology in order to determine the knowledge that should be included in the ontologies.

A2. The set of lexico-syntactic patterns analysed for identifying types of ontology requirements is representative and allows the classification of requirements. 
A3. Ontological requirements can be translated into tests.

A4. The adoption of Software Engineering practices in the Ontology Engineering field benefits the monitoring and analysis of the ontology development.

A5. Knowledge representation requirements of standards are defined accurately and unambiguously so that they can be used to identify differences and overlaps with ontologies based on them.

Once the assumptions have been identified and presented, the set of hypotheses of this thesis are described:

H1. Using a testing language for defining tests based on functional requirements facilitates the ontology testing process regarding the reduction of errors in users that are familiar and expert in OWL.

H2. There is a direct correlation between some metrics related to functional requirements and those metrics related to the implementation and the tests of the ontology.

H3. The majority of functional requirements shared by a set of ontologies from the same domain are related to the definition of terms and to the existence of relations between classes, while more restrictive requirements such as cardinalities are not frequently shared.

Finally, the following restrictions define the limits of the contributions of this thesis and allow to determine future research problems. These restrictions delimit the research problem and allow the incremental improvement of research:

R1. The verification and conformance of non-functional ontological requirements are out of scope.

R2. The ontology verification testing method only considers OWL 2 DL ontologies.

R3. Keywords for defining tests are written in the English language.

R4. Ontology requirements collected for the identification of lexico-syntactic patterns are written in the English language. 
R5. New keywords can appear and could be added to the testing language in the future.

R6. New lexico-syntactic patterns and, therefore, types of requirements can appear and could be added to the testing process in the future.

R7. The ontology testing method only considers ontologies that are syntactically correct, consistent and without unsatisfiable classes.

R8. The proposed metrics are only related to the artefacts generated and used during the ontology verification process, i.e., the ontology implementation, the ontology requirements specification document and the ontology requirements test suite.

R9. The roles in Ontology Engineering identified for defining the metrics only include: team leaders, ontology engineers and stakeholders. New roles can appear and could be added in the future.

R10. The ontology requirement test suite used to extract metrics is focused on tests extracted from requirements.

R11. The evaluation of the ontology conformance method only covers the Internet of Things (IoT) domain.

Figure 3.1 summarises the objectives identified in Section 3.1 and the contributions developed in this thesis presented in Section 3.2. The figure also includes, for each contribution, relations to the associated assumptions, hypothesis and restrictions.

\subsection{Evaluation plan}

With respect to the hypotheses defined in this thesis, in order to accept or reject them the following evaluations were performed (see Chapter 7):

E1 (for H1). The goal of this evaluation is to determine whether there is a benefit in the use of the proposed testing framework based on lexico-syntactic patterns. To this end, an empirical evaluation was carried out to compare several testing approaches in the state of the art with the testing approach proposed in this thesis for verifying an ontology. 


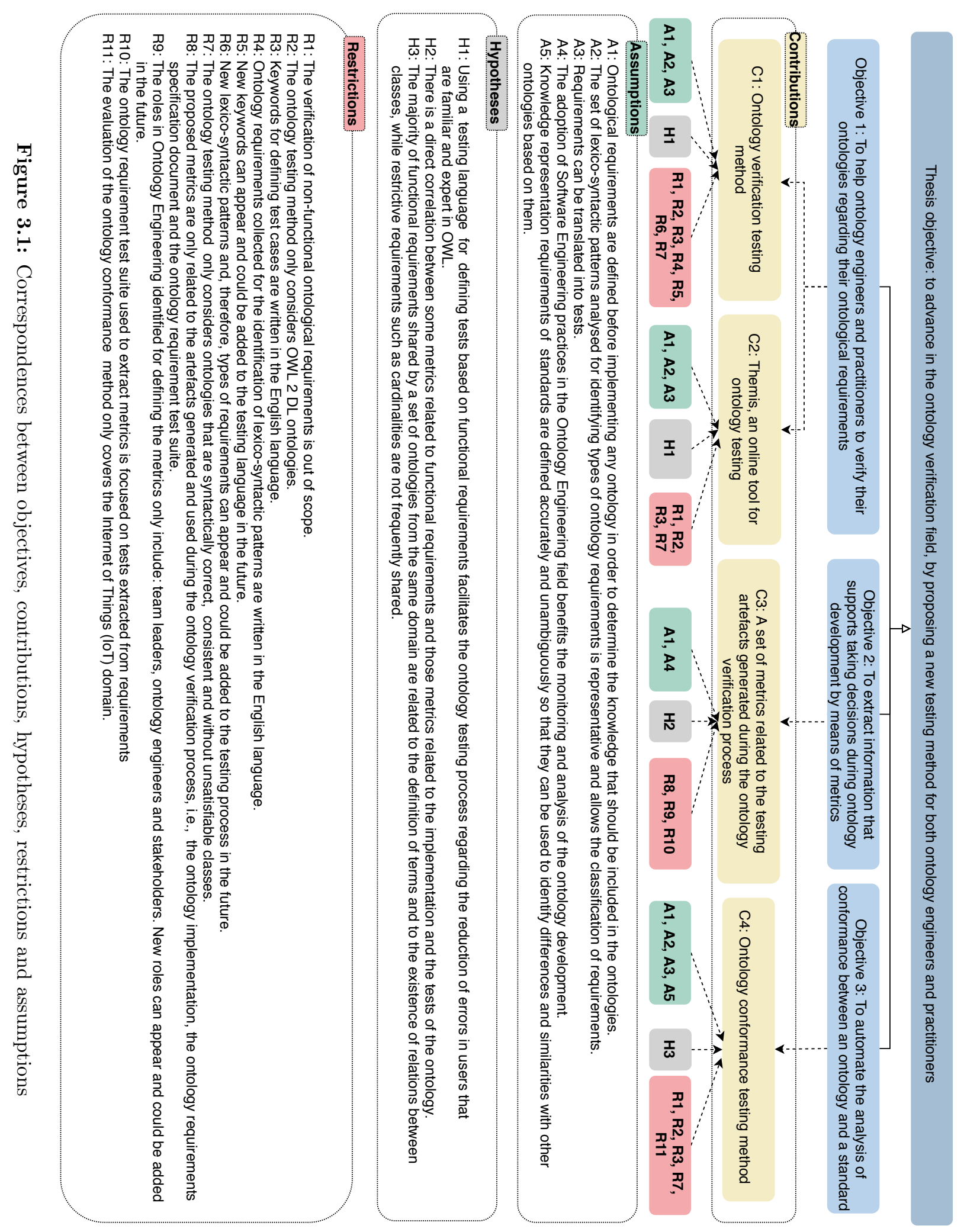


E2 (for H2). The goal of this evaluation is to analyse the metrics related to requirements, the ontology implementation and the tests, such as the complexity of requirements or the number of tests in the test suite, in order to determine if there is a correlation between them. To that end, the metrics described in this thesis were applied to a real-world project at different moments during the ontology development to examine such correlation.

E3 (for H3). The goal of this evaluation is to determine the types of functional requirements that are shared between an ontology and a standard related to the same domain. To that end, the conformance between them, as well as the minimum common knowledge between the standards, is analysed.

\subsection{Research methodology}

This section presents an overview of the research methodology followed during the development of this thesis. The approach for achieving the objectives of this thesis consists of providing several contributions to the open research problems defined in Section 3.1. These contributions are then combined to achieve each specified goal.

In order to deliver the contributions of this thesis, both methodological and technological inputs are considered, as it is shown in Figure 3.2. Additionally, community feedback has also been taken into account to validate and guide this thesis. Figure 3.2 also shows that the work is framed by a set of objectives, restrictions and assumptions.

For developing the ontology testing method methodological and technological support was taken into account:

1. Existing development methodologies in Ontology Engineering. Several ontology development methodologies, such as On-To-Knowledge (Staab et al., 2001), EXtreme ontology (Hristozova and Sterling, 2002), eXtreme design (Presutti et al. 2009) and SAMOD (Peroni, 2016), were analysed in order to extract how they deal with ontological requirements.

2. Existing testing approaches in Ontology Engineering. This thesis is grounded on the current approaches for ontology testing, both on methodologies and existing tools: 


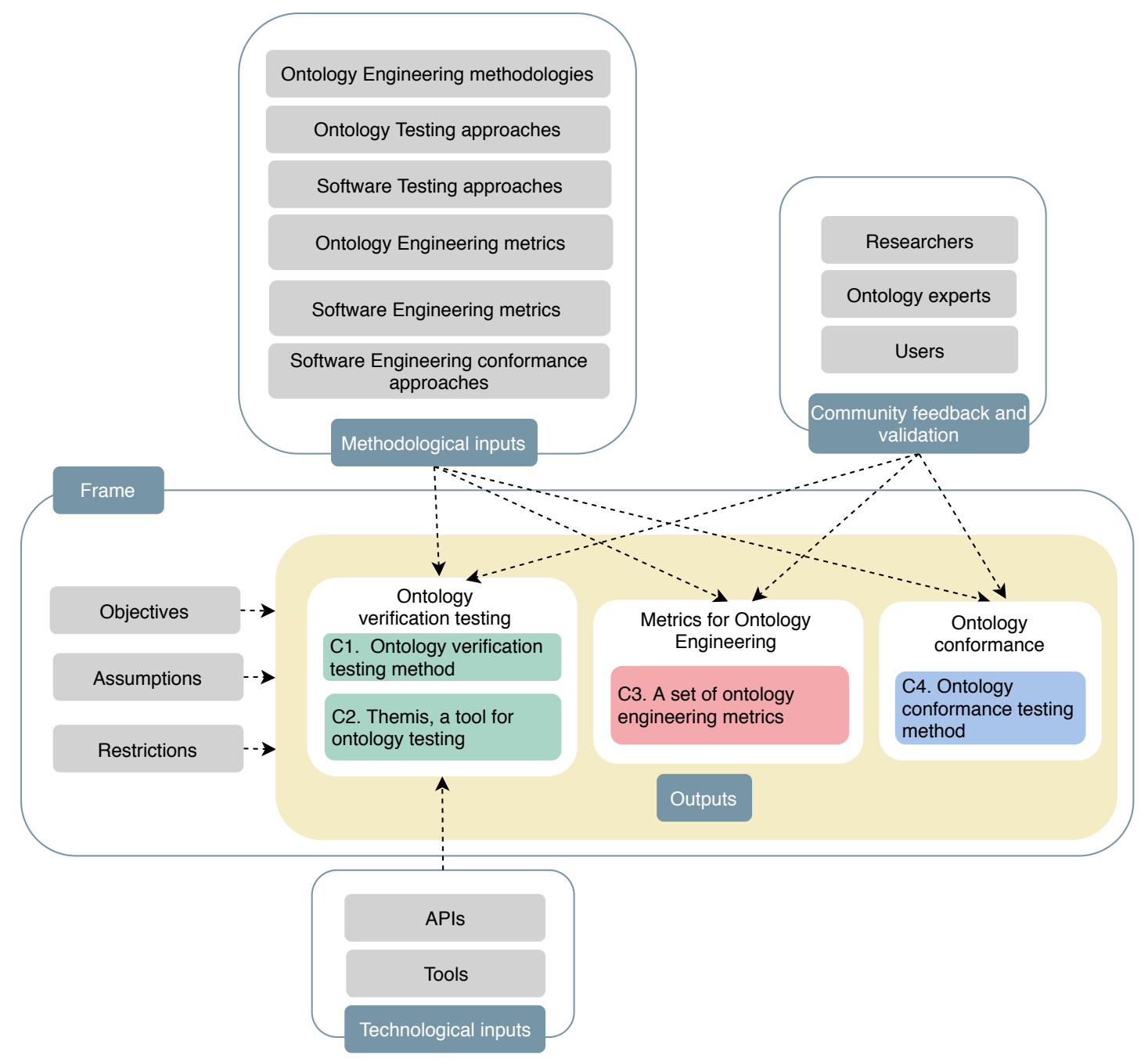

Figure 3.2: Inputs and context considered for creating the testing framework

- Methodologies. In this thesis several works are taken into account, including: (a) the work presented by Keet and Lawrynowicz as test-driven development (TDD) of ontologies (Keet and Ławrynowicz, 2016), in which the competency questions are formalised into axioms and added to the ontology if they are not present; (b) the approach introduced by Ren and colleagues (Ren et al. 2014) which uses natural language processing to analyse competency questions written in controlled natural language from where they create competency question patterns that could be automatically tested in the ontology; and (c) the agile approach presented by Blomqvist and col- 
leagues (Blomqvist et al., 2012) which includes a methodological background and introduces several types of tests concerned with the verification of the requirements implementation and the exposure to faults.

- Tools. Several tools were also reviewed, such as OntologyTest García-Ramos et al. 2009) and TDDOnto2 tool 15 which is a Protégé plugin to support the TDD methodology.

3. Available software. Available APIs for parsing RDF such as JENA ${ }^{16}$ and semantic reasoners such as Pellet (Sirin et al., 2007) were reused for the development of Themis.

Regarding the metrics for Ontology Engineering, this thesis is grounded on the following inputs:

1. Existing metrics in Software Engineering. The set of metrics proposed in this thesis is grounded in the multiple metrics proposed in the Software Engineering field. In this field, metrics analyse all the artefacts generated during the development process, such as the requirements specification (Fenton and Neil, 2000$)$ or the user documentation (Sommerville, 2010).

2. Existing metrics in Ontology Engineering. Existing metrics in Ontology engineering were also reviewed, even though they are mainly focused on the ontology structure. Such existing metrics analyse how the classes (Gangemi et al., 2006), properties (Tartir et al. 2005) and instances (Tartir et al., 2005) are organised and used in an ontology.

Finally, for developing the conformance testing method the following methodological input was taken into account:

1. Existing conformance approaches used in Software Engineering and in industry. The conformance approach proposed in this thesis is grounded on the current approaches that are used in Software Engineering and in industry. These approaches are developed by standardisation bodies such as ISO/IEC (ISO, 1994), ETSI (Moseley et al., 2003) and W3C (Dardailler, 2002).

\footnotetext{
${ }^{15}$ https://github.com/kierendavies/tddonto2

16 https://jena.apache.org/documentation/ontology/
} 


\subsubsection{Description of the research process}

In the development of this thesis, an incremental process was followed leading to four phases, namely: initialisation, development, implementation and refinement. Figure 3.3 shows an overview of the main contributions of each phase including: (a) the order in which the phases were carried out, (b) the tasks carried out during each phase, and (c) the publications obtained from each phase. Figure 3.3 intends to be self-contained and, therefore, the citations represented by integers between brackets (e.g., "[1]") correspond to the enumerated bibliographical resources at the bottom of the figure.

As described in Figure 3.2, in each phase of the development of the thesis several methodological and technological inputs were used. Along this section, the four phases are described:

1. Initialisation. During this phase, existing testing methodologies and tools were analysed, as well as ontology development methodologies that introduced the notion of testing. As a result, the initial version of the proposed testing approach was developed (related to $C 1$ ).

2. Development. During this phase, a set of 19 different types of tests were defined, together with the methods to implement them (related to C1). Furthermore, the initial version of the testing approach developed in the previous phase was improved and extended (related to C1). Finally, existing metrics in Ontology and Software Engineering were analysed. As a result, a set of ontology metrics related to the different artefacts generated during the development process was proposed (related to C3).

3. Implementation. During this phase, existing software libraries were used in order to implement Themis, the tool created to support the proposed testing approach (related to C2). Additionally, the set of tests defined in the previous phase was extended (related to $\mathrm{C} 1$ ). Moreover, the list of metrics was also extended (related to C3). Finally, an initial version of the conformance testing approach was developed (related to $C_{4}$ ).

4. Refinement. During this phase, continuous refinements and improvements were performed over the main contributions of this thesis. 


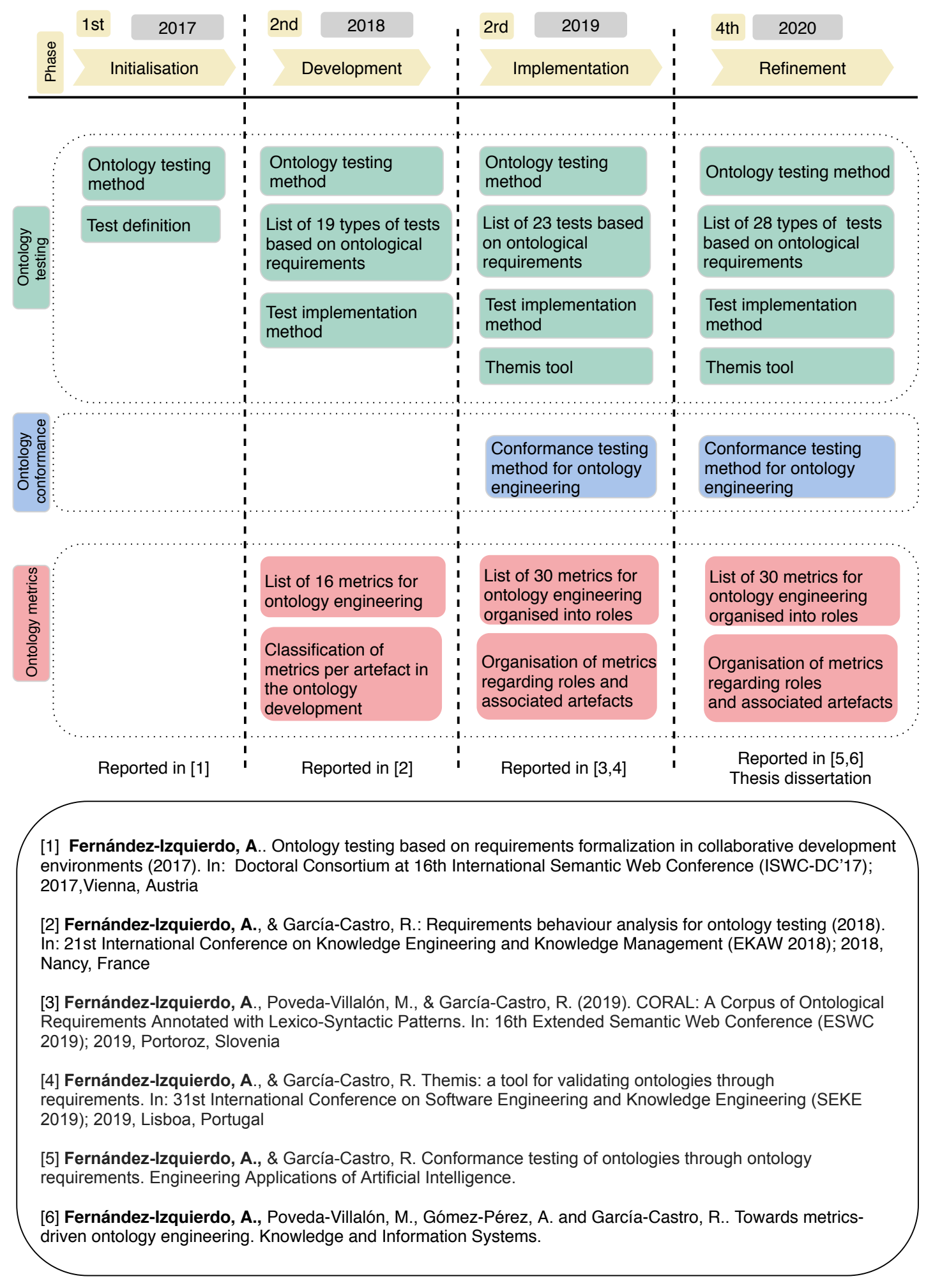

Figure 3.3: Phases of the thesis development, including the tasks carried out during each phase and the main publications derived from each phase. 



\section{Chapter 4}

\section{Method for ontology verification testing}

This chapter presents the method for ontology verification testing, together with its technological support. This method aims at involving domain experts and users in the ontology verification activity. However, if these actors are involved in the verification activity, it is necessary to use a language understandable by them. Therefore, inspired by software testing approaches, this testing method proposes a testing language based on how requirements are specified in order to facilitate the definition of tests.

Furthermore, this testing method aims at systematising the design, implementation and execution of tests extracted from ontology requirements in order to verify an ontology, as well as at providing traceability between ontology requirements, tests and ontologies.

Section 4.1 focuses on describing the analysis of ontology requirements to determine how they are specified, while Section 4.2 details the activities needed to design, implement and execute the tests based on such ontology requirements analysis. Section 4.3 describes the ontology built to store the tests and to allow their traceability and reuse. Finally, Section 4.4 presents Themis, the technological support for this testing method.

\subsection{Ontology requirements specification analysis}

In recent years, the definition of ontology requirements (Bezerra et al., 2013; SuárezFigueroa et al., 2015, 2009b), which represent the needs that the ontology to be built 
should cover, and their automatic formalisation into axioms or tests (Dennis et al. 2017, Ren et al., 2014; Zemmouchi-Ghomari and Ghomari, 2013) have been studied. These studies aim to reduce the time consumed by ontology engineers during the ontology verification activity. Ontology requirements can be written in the form of competency questions (Grüninger and Fox, 1995), which are natural language questions that the ontology to be modelled should be able to answer, or as statements.

However, to accurately define ontology requirements is not a trivial task and, therefore, neither is their automatic translation into a formal language. Due to the fact that some requirements are ambiguous (Montiel-Ponsoda, 2011) or vague, their transformation into axioms or tests is not usually direct (Dennis et al., 2017) and, consequently, it is challenging to automate such translation. Therefore, this thesis benefits from the use of Lexico-Syntactic Patterns (LSPs), which represent linguistic schemas or constructions derived from regular expressions in natural language. These LSPs consist of certain linguistic and para-linguistic elements which permit to extract conclusions about the meaning expressed by the construction (Aguado De Cea et al. 2008). Therefore, they are used to analyse how requirements are specified, with the ultimate goal of identifying types of tests based on them.

To analyse how ontology requirements are specified following LSPs, CORAL (Corpus of ontology requirements Annotated with Lexico-syntactic patterns), a corpus of 834 functional ontology requirements collected from different projects, websites and papers, has been created. CORAL aims at providing a resource to help the formalisation of ontology requirements in an ontology by providing a dictionary of LSPs, which includes those LSPs collected from the state of the art and also those new ones identified based on the requirements gathered in CORAL. Moreover, it provides a potential implementation of each LSP in an ontology. This dictionary of LSPs has been used to annotate the set of 834 ontology requirements in order to determine the LSPs that these requirements follow.

These LSPs identify structures in the specifications of ontology requirements; consequently, each LSP represents a particular type of requirement.

\subsubsection{Building the CORAL corpus}

This section is focused on describing the set of five steps that were followed to generate the CORAL corpus, which is summarised in Figure 4.1, as well as the availability of 
this corpus.


Figure 4.1: Steps, with their inputs and outputs, carried out to conduct the analysis and to create CORAL.

The five steps are the following:

1. To search for ontology requirements. A set of 834 functional requirements, which have their corresponding ontology implementation available, was collected. These requirements were written as competency questions and as statements, and collected from several projects, as well as from papers and from resources available on the Web. These 834 functional requirements were associated with 14 different ontologies, whose names and requirements provenance are listed in Table 4.1. These ontologies are built by different authors, and cover different topics and sizes. 
Table 4.1: Collected ontologies together with their requirements that have been analysed to create CORAL

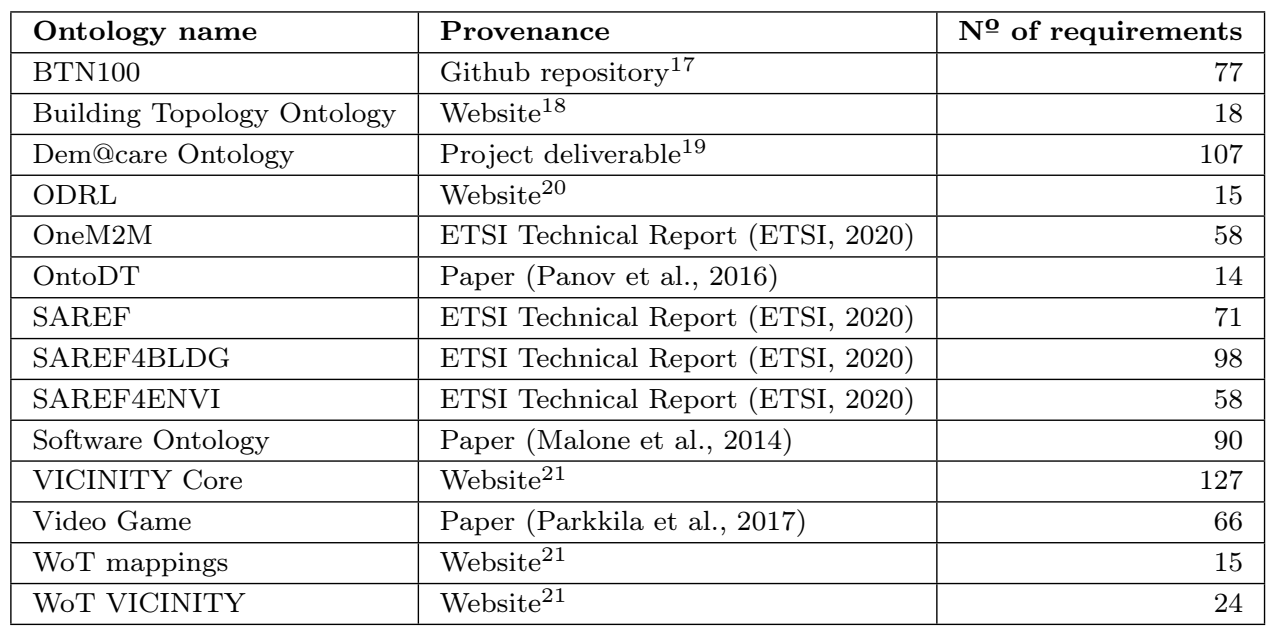

2. To search for existing LSPS. In order to annotate the corpus of ontology requirements, a dictionary of LSPs was created. Daga and colleagues (Daga et al. 2010 ) proposed a corpus of 17 LSPs ${ }^{22}$ each of which has one or more ontology design patterns (ODPs) (Gangemi and Presutti, 2009) associated that indicates how the LSP should be implemented in an ontology. It is possible that one LSP is associated with several disjoint ODPs, resulting in several possible ontology implementations. These LSPs are going to be called throughout this thesis polysemous LSPs. Besides the ODPs, each LSP is also associated with: a) an identifier, b) a formal representation according to a BNF extension ${ }^{23}$, and c) examples of sentences in natural language which match with such LSP. It should be mentioned that there can be more than one possible formalisation for each LSP.

3. To assign OWL constructs to existing LSPs. Based on the ODPs associated with each LSP, the set of OWL constructs needed to implement each LSP was identified.

The information related to how each ODP should be implemented was extracted

\footnotetext{
17 https://github.com/oeg-upm/ontology-BTN100/tree/master/requirements

18 https://w3c-lbd-cg.github.io/bot/\#Requirements

19 http://www.demcare.eu

20 http://w3c.github.io/poe/ucr/

${ }^{21}$ http://vicinity.iot.linkeddata.es

22 http://ontologydesignpatterns.org/wiki/Submissions: LexicoSyntacticoDPs

${ }^{23}$ https://www.w3.org/Notation.html
} 
from the NeOn project (Suárez-Figueroa et al., 2007), which defines a repository for ODPs along with their formal representation in an ontology. It was decided to consider every OWL construct except for the annotation and versioning ones, because they do not add expressivity to the ontology and that they are used as metadata. Considering that in some cases the LSPs can be associated with several disjoint ODPs and, therefore, implemented in alternative models, each LSP has different OWL constructs for each alternative model.

4. To assign expressivity to existing LSPs. According to the previously identified OWL constructs, the DL expressivity (Baader et al., 2008) of each LSP was determined. Because of the fact that some LSPs can be implemented into several models involving different OWL constructs, different expressivity has been associated with each model.

5. To annotate ontology requirements with LSPS. The annotation of ontology requirements is divided into two sub-steps:

5.1. To associate requirements and LSPs. The association between ontology requirements and LSP was manually made based on the syntax of each requirement and on its mapping with the formalisation of each LSP.

5.2. To define new LSPs. For those ontology requirements which did not match any of the state of the art LSPs, new patterns were provided to support them. These new patterns include the same information as the state of the art LSPs, such as the formalisation, associated ODPs (if exist) and examples of use. Altogether, a set of 12 new LSPs were added to the dictionary of patterns. If there are no ODPs associated with the LSP, then the OWL constructs were determined based on how each type of requirement is usually implemented in ontologies. As an example, a requirement related to a union between two concepts will be related to the OWL construct related to union, which is http://www.w3.org/2002/07/owl\#unionOf.

As a result of these steps, two results were obtained: (1) the dictionary of LSPs and (2) the corpus of annotated requirements. Both the dictionary of LSPs and the 
annotated corpus are openly available in HTML ${ }^{24}$ CSV and RDF format as Zenodo resources. They have a canonical citation using the DOI https://doi.org/10.5281/ zenodo.1967306 and are published under Creative Commons Attribution 4.0 International license (CC BY 4.0) ${ }^{25}$ Additionally, the corpus is also available in DataHul ${ }^{26}$ and from Google Dataset search.

\subsubsection{Dictionary of lexico-syntactic patterns}

The dictionary of LSPs collects the LSPs found in the state of art and those proposed during the development of the CORAL corpus. One LSP may be associated with several disjoint ODPs, resulting in several possible ontology implementations and, therefore, needing a different group of OWL constructs for each one. The translation from these polysemous LSPs to a formal implementation is not direct. Consequently, in such cases, the ontology engineers should decide which possible implementation they prefer according to the ontology they are implementing.

In this dictionary, the LSPs are categorised according to their correspondences, which could also be used to identify whether the LSP patterns are polysemous:

- 1 to 1 correspondence. One LSP corresponds to one possible implementation in the ontology.

- 1 to $\mathbf{N}$ correspondence. One LSPs can result in more than one possible implementation in the ontology. The LSPs that have more than 1 correspondence are considered polysemous LSPs.

The dictionary of LSPs is organised in groups according to the correspondence type, i.e., 1 to 1 or 1 to $N$. Table 4.2 summarises the LSPs according to their correspondence to ontology axioms. Such table also includes the source from where each LSPs has been extracted since some of them were defined in previous works (Daga et al., 2010).

\footnotetext{
$2^{24}$ http://coralcorpus.linkeddata.es

25 https://creativecommons.org/licenses/by/4.0

26 https://datahub.io/albaizq13/coralcorpus
} 
Table 4.2: List of lexico-syntactic patterns included in the dictionary

\begin{tabular}{|c|c|}
\hline LSP & Type of correspondence \\
\hline \multicolumn{2}{|r|}{1 to 1 correspondence } \\
\hline LSP-SC-EN & LSP for subclassOf relation ODP (Daga et al., 2010), \\
\hline LSP-MI-EN & LSP for multiple inheritance ODP (Daga et al., 2010), \\
\hline LSP-EQ-EN & LSP for equivalence relation ODP (Daga et al., 2010 ), \\
\hline LSP-OP-EN & LSP for object property ODP (Daga et al., 2010) \\
\hline LSP-DP-EN & LSP for datatype property ODP (Daga et al., 2010 ), \\
\hline LSP-Di-EN & LSP for disjoint classes ODP (Daga et al., 2010) \\
\hline LSP-SV-EN & LSP for specified values ODP (Daga et al., 2010 , \\
\hline LSP-PA-EN & LSP for participation ODP (Daga et al., 2010) \\
\hline LSP-PCP-EN & LSP for co-participation ODP (Daga et al., 2010, \\
\hline LSP-LO-EN & LSP for location ODP (Daga et al., 2010, \\
\hline LSP-OR-EN & LSP for object-role ODP (Daga et al., 2010) \\
\hline LSP-DC-SC-EN & LSP for defined classes and subclass (Fernández-Izquierdo et al., 2019) \\
\hline LSP- SC-Di-EN & $\begin{array}{l}\text { LSP for subclass relation, disjoint classes and exhaustive classes (Fernández- } \\
\begin{array}{|l|}\text { Izquierdo et al., 2019, }\end{array}\end{array}$ \\
\hline LSP-OP-UR-EN & $\begin{array}{l}\text { LSP for object property and universal restriction Fernández-Izquierdo et al. } \\
2019 \text {. }\end{array}$ \\
\hline LSP-CD-EN & LSP for class definition (Fernández-Izquierdo et al., 2019) \\
\hline LSP-Min-and-OP-EN & $\begin{array}{l}\text { LSP for object property minimum cardinality and object property } \\
\text { (Fernández-Izquierdo et al., 2019), }\end{array}$ \\
\hline LSP-OP-Min-EN & $\begin{array}{l}\text { LSP for object property minimum cardinality related to an object property } \\
\text { Fernández-Izquierdo et al., 2019), }\end{array}$ \\
\hline LSP-OP-Max-EN & $\begin{array}{l}\text { LSP for object property maximum cardinality related to an object property } \\
\text { Fernández-Izquierdo et al., 2019) }\end{array}$ \\
\hline LSP-OP-Exact-EN & $\begin{array}{l}\text { LSP for object property exact cardinality related to an object property } \\
\text { Fernández-Izquierdo et al., 2019) }\end{array}$ \\
\hline LSP-SYM-EN & LSP for symmetry (Fernández-Izquierdo et al., 2019), \\
\hline LSP-U-EN & LSP for union Fernández-Izquierdo et al., 2019 \\
\hline LSP-INTER-EN & LSP for intersection (Fernández-Izquierdo et al., 2019) \\
\hline LSP-COMPL-EN & LSP for complement (Fernández-Izquierdo et al.,, 2019 , \\
\hline \multicolumn{2}{|r|}{1 to $\mathrm{N}$ correspondences } \\
\hline LSP-SC-PW-EN & $\begin{array}{l}\text { LSP for subclass or simple part-whole relation Fernández-Izquierdo et al., } \\
2019 \text {, }\end{array}$ \\
\hline LSP-OP-DP-PW-EN & $\begin{array}{l}\text { LSP for object property or datatype property or simple part-whole relation } \\
\text { (Fernández-Izquierdo et al., 2019), }\end{array}$ \\
\hline LSP-PW-CONS-COM-CE-EN & $\begin{array}{l}\text { LSP for simple part-whole relation or constituency or componency or } \\
\text { collection-entity (Fernández-Izquierdo et al., 2019) }\end{array}$ \\
\hline LSP-DC-SC-EN & $\begin{array}{l}\text { LSP for defined classes and subclass relation (Fernández-Izquierdo et al., } \\
2019 \text {. }\end{array}$ \\
\hline LSP-INST-SC-EN & LSP for instances of subclass relation Fernández-Izquierdo et al., 2019) \\
\hline LSP-OP-DP-EN & $\begin{array}{l}\text { LSP for object property or datatype property Fernández-Izquierdo et al. } \\
2019 \text {, }\end{array}$ \\
\hline
\end{tabular}

In the dictionary, additionally to the type of pattern, each LSP is associated with its corresponding OWL constructs, as it is summarised in Table 4.3 . 
Table 4.3: OWL constructs and DL expressivity associated with each lexico-syntactic pattern

\begin{tabular}{|c|c|c|}
\hline LSP & OWL constructs & DL Expressivity \\
\hline \multicolumn{3}{|c|}{1 to 1 correspondence } \\
\hline LSP-SC-EN & rdfs:subclassOf, owl:Class & $\mathrm{AL}$ \\
\hline LSP-MI-EN & rdfs:subclassOf, owl:Class & $\mathrm{AL}$ \\
\hline LSP-EQ-EN & owl:equivalentClass, owl:Class & $\mathrm{AL}$ \\
\hline LSP-OP-EN & owl:Class, owl:someValuesFrom, owl:ObjectProperty & ALE \\
\hline LSP-DP-EN & owl:Class, owl:someValuesFrom, owl:DatatypeProperty & $\mathrm{ALE}(\mathrm{D})$ \\
\hline LSP-Di-EN & owl:Class, owl:disjointWith & ALC \\
\hline LSP-SV-EN & owl:Class, owl:NamedIndividual & $\mathrm{AL}$ \\
\hline LSP-PA-EN & $\begin{array}{l}\text { owl:Class, owl:ObjectProperty, owl:someValuesFrom, } \\
\text { owl:inverseOf }\end{array}$ & ALEI \\
\hline LSP-PCP-EN & $\begin{array}{l}\text { owl:Class, owl:ObjectProperty, owl:someValuesFrom, } \\
\text { owl:SymmetricProperty }\end{array}$ & ALEI \\
\hline LSP-LO-EN & owl:Class, owl:ObjectProperty, owl:someValuesFrom & ALE \\
\hline LSP-OR-EN & owl:Class, owl:ObjectProperty, owl:allValuesFrom & $\mathrm{AL}$ \\
\hline LSP-DC-SC-EN & owl:Class, rdfs:subclassOf & $\mathrm{AL}$ \\
\hline LSP- SC-Di-EN & owl:Class, rdfs:subclassOf, owl:disjointWith & ALC \\
\hline LSP-OP-UR-EN & $\begin{array}{l}\text { owl:Class, owl:ObjectProperty, rdfs:subclassOf, } \\
\text { owl:allValuesFrom }\end{array}$ & $\mathrm{AL}$ \\
\hline LSP-CD-EN & owl:Class & $\mathrm{AL}$ \\
\hline LSP-Min-and-OP-EN & owl:Class, owl:ObjectProperty, owl:minCardinality & ALEQ \\
\hline LSP-OP-Min-EN & owl:Class, owl:ObjectProperty, owl:minCardinality & ALEQ \\
\hline LSP-OP-Max-EN & owl:Class, owl:ObjectProperty, owl:maxCardinality & ALEQ \\
\hline LSP-OP-Exact-EN & owl:Class, owl:ObjectProperty, owl:cardinality & ALEQ \\
\hline LSP-SYM-EN & owl:SymmetricProperty, owl:ObjectProperty, owl:Class & ALI \\
\hline LSP-U-EN & owl:unionOf, owl:ObjectProperty, owl:Class & $\mathrm{ALU}$ \\
\hline LSP-INTER-EN & owl:ObjectProperty, owl:Class, owl:intersectionOf & ALE \\
\hline LSP-COMPL-EN & $\begin{array}{l}\text { owl:Class, owl:ObjectProperty, owl:someValuesFrom , } \\
\text { owl:complementOf }\end{array}$ & ALC \\
\hline \multicolumn{3}{|c|}{1 to $\mathrm{N}$ correspondences } \\
\hline \multirow{3}{*}{ LSP-SC-PW-EN } & owl:Class & $\mathrm{AL}$ \\
\hline & owl:Class, rdfs:subclassOf & $\mathrm{AL}$ \\
\hline & $\begin{array}{l}\text { owl:Class, } \\
\text { owl:inverseOf }\end{array}$ & ALEI \\
\hline \multirow{3}{*}{ LSP-OP-DP-PW-EN } & owl:Class, owl:ObjectProperty, owl:someValuesFrom & ALE \\
\hline & owl:Class, owl:DatatypeProperty, owl:allValuesFrom & ALE(D) \\
\hline & $\begin{array}{l}\text { owl:Class, owl:ObjectProperty, owl:allValuesFrom, } \\
\text { owl:inverseOf }\end{array}$ & ALEI \\
\hline LSP-PW-CONS-COM-CE-EN & $\begin{array}{l}\text { owl:Class, owl:ObjectProperty, owl:inverseOf, } \\
\text { owl:someValuesFrom }\end{array}$ & ALEI \\
\hline \multirow{3}{*}{ LSP-DC-SC-EN } & owl:Class, rdfs:subclassOf & $\mathrm{AL}$ \\
\hline & owl:Class & $\mathrm{AL}$ \\
\hline & owl:Class, owl:DatatypeProperty, owl:maxCardinality & ALEQ(D) \\
\hline \multirow{2}{*}{ LSP-INST-SC-EN } & owl:Class,rdfs:subclassOf & $\mathrm{AL}$ \\
\hline & owl:NamedIndividual, owl:Class & $\mathrm{AL}$ \\
\hline \multirow[t]{2}{*}{ LSP-OP-DP-EN } & $\begin{array}{lll}\text { owl:ObjectProperty, } & \text { rdfs:subclassOf, } & \text { owl:Class, } \\
\text { owl:someValuesFrom } & \end{array}$ & ALE \\
\hline & $\begin{array}{l}\text { owl:DatatypeProperty, } \quad \text { rdfs:subclassOf, } \\
\text { owl:someValuesFrom }\end{array}$ & ALE(D) \\
\hline
\end{tabular}


These OWL constructs have been extracted from the ODPs since they indicate how these patterns should be implemented in the ontology. As mentioned before, if an LSP is not associated with at least one ODP then the OWL constructs are determined based on how such LSP is usually implemented in ontologies. Additionally, each LSP is also linked with its DL expressivity, which was extracted from the OWL constructs associated with it.

\subsubsection{Annotated corpus of ontology requirements}

As mentioned in Section 4.1.2, a corpus of 834 requirements has been annotated with the LSPs defined in the dictionary of LSPs. These annotations determine how each requirement is specified and, consequently, how it can be formalised into an ontology. Table 4.4 summarises the results obtained from the annotation process, indicating how many requirements have been associated with each LSP and providing an overview of how requirements used to be specified.

From Table 4.4 it can be observed that the most common LSP in requirements specifications is the LSP-OP-EN, which is related to object properties. Other popular LSPs are the LSP-OP-DP-EN and the LSP-OP-DP-PW-EN, which are polysemous LSPs and, therefore, they do not have a direct translation to axioms or tests. It should be taken into account that there are several requirements that are associated with several LSPs, due to the fact that they include several sentences. Therefore, the sum of the values in columns in Figure 4.4 is higher than 834.

\subsection{Activities within the ontology verification process}

This section details the activities to be carried out during the testing process for verifying an ontology. In the literature, ontology testing approaches are usually divided into two activities, i.e., test implementation and test execution. However, based on the requirements specification analysis described in Section 4.1, in this testing method a new activity is proposed, i.e., test design. This new activity is needed due to the ambiguity and assumptions inherent to the natural language (Dennis et al., 2017) and to the fact that different people may be in charge of the design and implementation of tests. Additionally, the separation between the test design and the test implementation increases the maintainability of the tests, since the implementation can change without 
Table 4.4: Number of requirements annotated with each lexico-syntactic pattern

\begin{tabular}{|c|c|}
\hline LSP & № of annotated requirements \\
\hline \multicolumn{2}{|c|}{1 to 1 correspondence } \\
\hline LSP-OP-EN & 415 \\
\hline LSP-CD-EN & 38 \\
\hline LSP-OP-Min-EN & 21 \\
\hline LSP-SC-EN & 12 \\
\hline LSP-DP-EN & 7 \\
\hline LSP-OP-Exact-EN & 5 \\
\hline LSP- SC-Di-EN & 4 \\
\hline LSP-Min-and-OP-EN & 3 \\
\hline LSP-SYM-EN & 3 \\
\hline LSP-COMPL-EN & 2 \\
\hline LSP-OP-Max-EN & 2 \\
\hline LSP-INTER-EN & 1 \\
\hline LSP-LO-EN & 1 \\
\hline LSP-MI-EN & 0 \\
\hline LSP-EQ-EN & 0 \\
\hline LSP-Di-EN & 0 \\
\hline LSP-SV-EN & 0 \\
\hline LSP-PA-EN & 0 \\
\hline LSP-PCP-EN & 0 \\
\hline LSP-OR-EN & 0 \\
\hline LSP-DC-SC-EN & 0 \\
\hline LSP-OP-UR-EN & 0 \\
\hline \multicolumn{2}{|c|}{1 to $N$ correspondences } \\
\hline LSP-OP-DP-EN & 121 \\
\hline LSP-OP-DP-PW-EN & 118 \\
\hline LSP-DC-SC-EN & 46 \\
\hline LSP-INST-SC-EN & 17 \\
\hline LSP-PW-CONS-COM-CE-EN & 11 \\
\hline LSP-SC-PW-EN & 0 \\
\hline
\end{tabular}

updating the design of the test. Therefore, in this design activity the goal of each requirement is identified and specified using a testing language based on the types of requirements presented in Section 4.1

The following sections describe each of these testing activities, which are also summarised in Figure 4.2. The test design activity is a manual activity that should be performed by the ontology engineers and practitioners, while the test implementation and the test execution activities can be carried out automatically. 


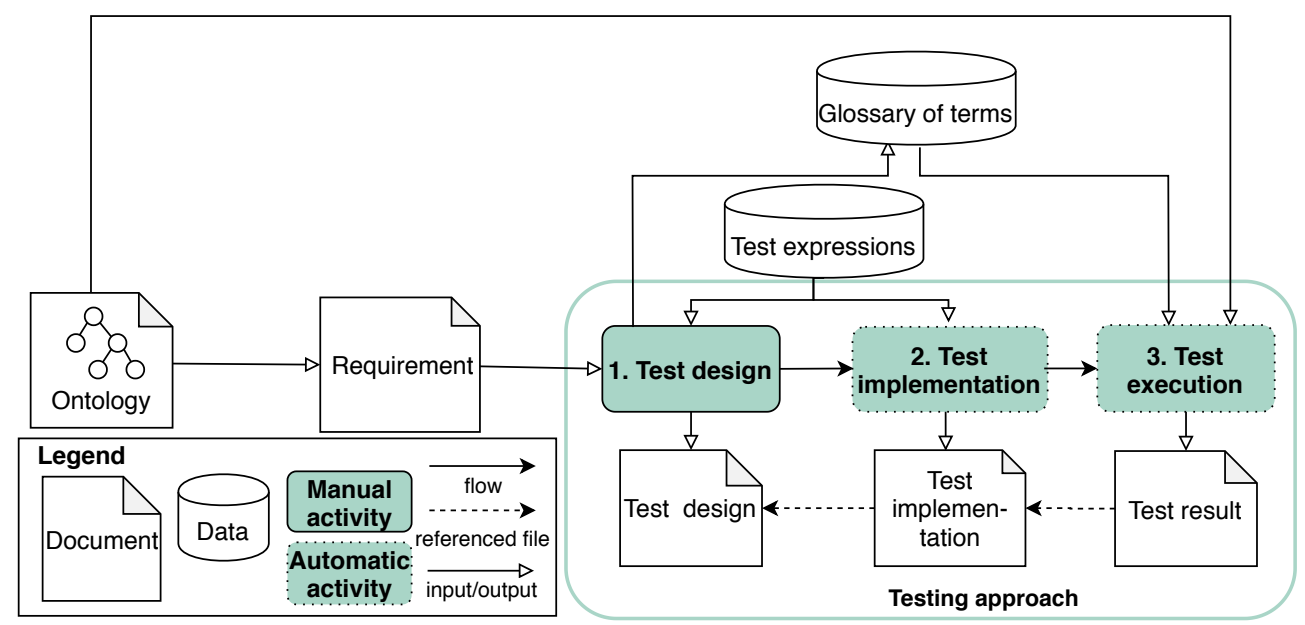

Figure 4.2: Testing activities during the testing process, together with their inputs and outputs

\subsubsection{Test design activity}

Ontology engineers, domain experts and users should be involved in the testing process for ontology verification, where tests are generated from the requirements in order to ensure that the ontology satisfies all the expected needs. However, writing tests in formal languages such as SPARQL, which is a language whose semantics are not easy to understand for people without a background on it (Pérez et al., 2009), is not a trivial process.

Inspired by Software Engineering techniques and languages to ease the test design process, such as keyword-driven testing, this thesis describes a testing language for designing tests. Since the LSPs introduced in Section 4.1.2 determine types of requirements and how they should be implemented in an ontology, the testing language is grounded on them.

To that end, representative keywords have been extracted from each LSP. These keywords, which indicate the goal in the ontology for each LSP, e.g., to define a subsumption relation or a cardinality restriction, are used to propose a catalogue of test expressions that are written following the OWL Manchester syntax 27. Therefore, each test refers to a particular type of requirement and includes a set of keywords, e.g., the

${ }^{27}$ https://www.w3.org/TR/owl2-manchester-syntax 
tests related to subsumption relations between classes includes the keyword subClas$s O f$. Since these tests are written using keywords, they can be automatically analysed and implemented into queries or axioms to be executed on the ontology, allowing test automation.

Due to the fact that polysemous LSPs do not have a direct translation from requirements into an ontology, since multiple ontology implementations can be correct, they are not considered for the time being. Additionally, it is worth mentioning that a small set of tests and keywords was also added to the catalogue by demand of ontology experts.

The catalogue of test expressions is shown in Table 4.5. The table includes the goal of each test, its syntax and its provenance. Those terms that are represented between brackets (e.g., "[Class]") correspond to those terms that should be completed by the user, while those terms italicised (e.g., type) correspond to the keywords that cannot be changed in the test. Table 4.5 is divided into three categories, according to the axioms analysed by each test:

- Simple tests. These tests are associated with a single axiom in an ontology. These axioms could be related to declarations of terms as well as to restrictions.

- Composed tests. These tests are associated with a combination of axioms in an ontology, which could be a combination of class axioms, descriptions or property axioms.

- ODP-based tests. These tests are associated with ODPs. Up to now, the ODPbased test expressions are associated with Content Ontology Design Patterns (CP) (Gangemi and Presutti, 2009), which propose patterns for solving design problems for the domain classes and properties that populate an ontology and, therefore, addressing content problems. Examples of these CPs are the Participation ${ }^{28}$ or the PartOf 29 ones.

- Usage tests. These tests are related to how the ontology is used, rather than checking a particular restriction in the ontology.

\footnotetext{
${ }^{28}$ http://ontologydesignpatterns.org/wiki/Submissions:Participation

${ }^{29}$ http://ontologydesignpatterns.org/wiki/Submissions:Partof
} 
Table 4.5: List of proposed test expressions together with their category

\begin{tabular}{|c|c|c|}
\hline Goal & Syntax & Source \\
\hline \multicolumn{3}{|c|}{ Simple test } \\
\hline T1. Class A exists & [ClassA] type Class & LSP-CD-EN \\
\hline $\begin{array}{l}\text { T2. Subsumption relation between classes } \\
\mathrm{A} \text { and } \mathrm{B}\end{array}$ & [ClassA] subclassOf [ClassB] & LSP-SC-EN \\
\hline T3. Disjointness between two classes & [ClassA] disjointWith [ClassB] & LSP-Di-EN \\
\hline T4. Equivalence between two classes & [ClassA] equivalentTo [ClassB] & LSP-EQ-EN \\
\hline T5. Property P exists & [Property] type Property & Ontology experts \\
\hline $\begin{array}{l}\text { T6. Existential relation } \mathrm{P} \text { between two } \\
\text { classes } \mathrm{A} \text { and } \mathrm{B}\end{array}$ & $\begin{array}{llll}\text { ClassA }] & \text { subclassOf } & {[\text { PropertyP }]} & \text { some } \\
{[\text { ClassB }]} & & & \end{array}$ & $\begin{array}{l}\text { LSP-OP-EN, LSP-DP- } \\
\text { EN, LSP-LO-EN, LSP- } \\
\text { SV-EN }\end{array}$ \\
\hline $\begin{array}{l}\text { T7. Universal relation } \mathrm{P} \text { between two } \\
\text { classes } \mathrm{A} \text { and } \mathrm{B}\end{array}$ & [ClassA] subclass Of [PropertyP] only [ClassB] & $\begin{array}{l}\text { LSP-OP-EN, LSP-DP- } \\
\text { EN, LSP-LO-EN, LSP- } \\
\text { SV-EN }\end{array}$ \\
\hline T8. Symmetric property $\mathrm{P}$ & [PropertyA] characteristic symmetric & LSP-SYM-EN \\
\hline T9. Minimum cardinality & $\begin{array}{l}\text { [ClassA] subclassOf }[\text { PropertyP] } \min \text { [num] } \\
\text { [ClassB] }\end{array}$ & LSP-OP-Min-EN \\
\hline T10. Maximum cardinality & $\begin{array}{l}\text { [ClassA] subclassOf [PropertyP] } \max \text { [num] } \\
\text { [ClassB] }\end{array}$ & LSP-OP-Max-EN \\
\hline T11. Cardinality & $\begin{array}{l}\text { [ClassA] subclassOf [PropertyP] exactly [num] } \\
\text { [ClassB] }\end{array}$ & LSP-OP-Exact-EN \\
\hline $\begin{array}{l}\text { T12. Universal relation } \mathrm{P} \text { between the } \\
\text { union of two classes } \mathrm{A} \text { and } \mathrm{B}\end{array}$ & $\begin{array}{l}\text { [ClassA] subclass Of [PropertyP] only [ClassB] } \\
\text { or }[\text { ClassC }]\end{array}$ & LSP-U-EN \\
\hline $\begin{array}{l}\text { T13. Universal relation } \mathrm{P} \text { between the in- } \\
\text { tersection of two classes } \mathrm{A} \text { and } \mathrm{B}\end{array}$ & $\begin{array}{lrrr}\text { ClassA }] & \text { subclassOf } & \text { [PropertyP }] & \text { some } \\
{[\text { ClassB] }} & \text { and }[\text { ClassC }] & & \\
\end{array}$ & LSP-INTER-EN \\
\hline T14. Individual I exists & [IndividualI] type [ClassA] & Ontology experts \\
\hline T15. Property $\mathrm{P}$ has domain class A & [PropertyP] domain [ClassA] & Ontology experts \\
\hline T16. Property $\mathrm{P}$ has range class $\mathrm{A}$ & [PropertyP] range [ClassA] & Ontology experts \\
\hline \multicolumn{3}{|c|}{ Composed tests } \\
\hline T17. Multiple inheritance of class A & [ClassA] subclassOf [ClassB] and [ClassC] & LSP-MI-EN \\
\hline $\begin{array}{l}\text { T18. Subsumption and relation between } \\
\text { classes }\end{array}$ & $\begin{array}{l}\text { [ClassA] subClassOf [ClassB] that [PropertyP] } \\
\text { some }[\text { ClassC }]\end{array}$ & LSP-DC-SC-EN \\
\hline $\begin{array}{l}\text { T19. Minimum cardinality between classes } \\
\mathrm{A} \text { and B, and existential relation P be- } \\
\text { tween classes B and C }\end{array}$ & $\begin{array}{l}\text { [ClassA] subclassof [PropertyP] } \min [\mathrm{num}] \\
{[\text { ClassB] and }[\text { ClassB] subclassof }[\text { PropertyP] }} \\
\text { some }[\text { ClassC }]\end{array}$ & LSP-Min-and-OP-EN \\
\hline $\begin{array}{l}\text { T20. Minimum cardinality between classes } \\
\text { A and B and universal relation P between } \\
\text { classes B and C }\end{array}$ & $\begin{array}{l}\text { [ClassA] subclassof [PropertyP] min }[\mathrm{num}] \\
{[\text { ClassB] and }[\text { ClassB] subclassof }[\text { PropertyP] }} \\
\text { only }[\text { ClassC }]\end{array}$ & LSP-Min-and-OP-EN \\
\hline $\begin{array}{l}\text { T21. Subsumption relation between A and } \\
\mathrm{B} \text {, subsumption relation between A and C, } \\
\text { and disjointness between B and C }\end{array}$ & $\begin{array}{l}\text { [ClassB] subclassOf }[\text { ClassA] and }[\text { ClassC] sub- } \\
\text { classOf }[\text { ClassA] that disjoint With [ClassB] }\end{array}$ & LSP- SC-Di-EN \\
\hline \multicolumn{3}{|c|}{ ODP-based tests } \\
\hline $\begin{array}{l}\text { T22. Participation ODP between classes } \\
\mathrm{A} \text { and } \mathrm{B}\end{array}$ & $\begin{array}{l}\text { [ClassA] subClassOf isPartici- } \\
\text { pantIn/hasParticipant some [ClassB] }\end{array}$ & LSP-PA-EN \\
\hline T23. Co-participation ODP & $\begin{array}{l}\text { [ClassA] and [ClassB] subClassOf isPartici- } \\
\text { pantIn/hasParticipant some [ClassC] }\end{array}$ & LSP-PCP-EN \\
\hline $\begin{array}{l}\text { T24. PartOf ODP between classes } \mathrm{A} \text { and } \\
\mathrm{B} \text {, with existential restriction }\end{array}$ & $\begin{array}{lll}\text { ClassA] subClassOf isPartof/hasPart some } \\
\text { [ClassB] }\end{array}$ & Ontology experts \\
\hline $\begin{array}{l}\text { T25. PartOf ODP between classes } \mathrm{A} \text { and } \\
\mathrm{B} \text {, with universal restriction }\end{array}$ & $\begin{array}{lll}\text { [ClassA] } & \text { subClassOf isPartof/hasPart only } \\
\text { [ClassB] } & & \\
\end{array}$ & Ontology experts \\
\hline $\begin{array}{l}\text { T26. Object-Role ODP between classes A } \\
\text { and } \mathrm{B} \text {, with existential restriction }\end{array}$ & $\begin{array}{l}\text { [ClassA] subClassOf isRoleOf/hasRole some } \\
\text { [ClassB] }\end{array}$ & LSP-OR-EN \\
\hline $\begin{array}{l}\text { T27. Object-Role ODP between classes A } \\
\text { and, with universal restriction }\end{array}$ & $\begin{array}{lll}\text { [ClassA] } & \text { subClassOf isRoleOf/hasRole only } \\
\text { [ClassB] } & & \\
\end{array}$ & LSP-OR-EN \\
\hline \multicolumn{3}{|c|}{ Usage tests } \\
\hline $\begin{array}{l}\text { T28. Two individuals of classes A and B } \\
\text { can be related by property } \mathrm{P}\end{array}$ & [ClassA] [PropertyP] [ClassB] & Ontology experts \\
\hline
\end{tabular}


As mentioned in Section 3.3, the list of tests does not pretend to be exhaustive and might be extended in the future with new ones.

During this activity, the goal of each requirement is extracted and formalised using the set of test expressions included in the catalogue described in Table 4.5. In order to create the test, each ontology requirement should be associated with at least one test expression presented in Table 4.5. Each test expression is related to a particular goal to be checked by such test, as shown in Table 4.5, which should concur with the goal of the requirement. A complex requirement that includes several sentences could be categorised with more than one LSP and, therefore, could be associated with more than one test.

The tests are defined without any information, e.g., URIs or labels, related to the ontology in which such test will be executed. With this separation between the test and the ontology, the reuse of tests in different ontologies is allowed. However, the terms included in the test must be present in the glossary of terms of the ontology on which the test will be executed, as shown in Figure 4.2. This glossary of terms aims at mapping these terms that are defined in the test design with terms in the ontology. The terms must follow camel case style, e.g., DigitalEntity.

As an example of test design, the requirement that states "An IoT gateway is a digital entity" has as goal to model a subsumption relation between two entities. Therefore, the test to be used should refer to "T2: Subsumption relation between classes A and B". Consequently, the test expression for T2 determined in the catalogue, i.e., "[ClassA] subClassOf [ClassB]", is completed with the information of the requirement. The requirement "An IoT gateway is a digital entity" is then associated with the test "Gateway subClassOf DigitalEntity", since Gateway and DigitalEntity are terms that describe the concepts in the test. Then, the terms Gateway and DigitalEntity must be included in the glossary of terms as keywords to be associated with terms in the ontology during the test execution activity, e.g., Gateway and DigitalEntity could be associated with «http://www.example.org/ontology/ns\#Gateway» and «http://www.example.org/ontology/ns\#DigitalEntity», respectively.

\subsubsection{Test implementation activity}

During this activity, the tests should be implemented in order to be executed on an ontology. To this end, each test is formalised into a precondition, a set of auxiliary term 
declarations and a set of assertions to check the behaviour.

The precondition is a SPARQL query which checks whether the terms involved in the ontology requirement are defined in the ontology. In order to execute the tests, these terms need to be declared in the ontology. Otherwise, the test fails and the requirement is not satisfied.

The axioms to declare auxiliary terms (i.e., test preparation) are a set of temporary axioms added to the ontology to declare the auxiliary terms needed to carry out the assertions.

Finally, the assertions to check the behaviour are a set of pairs of axioms and expected results that represent different ontology scenarios. For each pair, the axiom is temporarily added to the ontology to force a scenario, after which the reasoner is executed. The expected result determines if the ontology status (i.e., inconsistent ontology, unsatisfiable class or consistent ontology) after the addition is the expected one. If the status concurs with the expected status, then the requirement is satisfied.

Table 4.6 shows the test implementation associated with the test design for checking equivalence between classes (test expression with identifier T4 in Table 4.5). For the sake of readability, the axioms in these tables are represented by means of Description Logics syntax (Baader et al. 2008).

Table 4.6: T4. Equivalence between two classes A and B

\begin{tabular}{|c|c|c|c|}
\hline Goal: & \multicolumn{3}{|c|}{ T4. Equivalence between two classes A and B } \\
\hline Test expression: & \multicolumn{3}{|c|}{ [ClassA] equivalentTo [ClassB] } \\
\hline Type: & Simple test & Related to: & Classes \\
\hline \multicolumn{2}{|c|}{ Test precondition } & \multicolumn{2}{|c|}{ Test preparation } \\
\hline \multicolumn{2}{|c|}{ Class A and Class B exist } & \multicolumn{2}{|c|}{$\begin{array}{l}\text { (E 1.1) Declaration of } \neg \mathrm{A} \\
\text { (E 1.2) Declaration of } \neg \mathrm{B}\end{array}$} \\
\hline \multicolumn{4}{|c|}{ Assertions to test the ontology behaviour } \\
\hline \multicolumn{2}{|r|}{ Axiom } & \multicolumn{2}{|c|}{ Result } \\
\hline \multicolumn{2}{|c|}{ (E 2) Assertion $\mathrm{A}^{\prime} \sqsubseteq \neg \mathrm{A} \sqcap \mathrm{B}$} & \multicolumn{2}{|c|}{ Unsatisfiable class } \\
\hline \multicolumn{2}{|c|}{ (E 3) Assertion $\mathrm{A}^{\prime} \sqsubseteq \mathrm{A} \sqcap \neg \mathrm{B}$} & \multicolumn{2}{|c|}{ Unsatisfiable class } \\
\hline \multicolumn{2}{|c|}{ (E 4) Assertion $A^{\prime} \sqsubset \mathrm{A} \sqcap \mathrm{B}$} & \multicolumn{2}{|c|}{ Consistent ontology } \\
\hline
\end{tabular}

To check equivalence between two classes, a set of auxiliary terms are defined, i.e., the classes that complement $A$, i.e., $\neg A$ and $B$, i.e., $\neg B$. After their definition, a set of assertions that force the ontology to present unsatisfiable classes or inconsistencies are 
also defined. The first one, associated with axiom 'E 2' in Table 4.6, generates a class $A$ ' that is defined as a subclass of class $\mathrm{B}$ and $\neg A$. If the ontology satisfies the requirement, this addition causes an unsatisfiable class due to the fact that the reasoner would infer that $A$ ' is subclass of $A$ and $\neg A$. The second assertion, associated with axiom ' $\mathrm{E} 3$ ', generates a class $A^{\prime}$ that is defined as a subclass of class $A$ and $\neg B$. If the ontology satisfies the requirement, this addition causes an unsatisfiable class due to the fact that the reasoner would infer that $A^{\prime}$ is subclass of $B$ and $\neg B$. The last assertion, associated with axiom ' $\mathrm{E} 4$ ', generates a class $A$ ' that is defined as a subclass of class $A$ and $B$. If the ontology satisfies the equivalence requirement, this assertion causes a consistent ontology due to the fact that there is no problem if $A^{\prime}$ is subclass of $A$ and $B$.

Annex A shows the test implementations for all the test expressions included in Table 4.5, which follow the same structure as Table 4.6 .

\subsubsection{Test execution activity}

Taking as input the test implementation, the test execution activity consists of three steps: (1) the execution of the query that represents the preconditions, (2) the addition of the axioms that declare the auxiliary terms, and (3) the addition of the assertions. After the addition of each axiom, a reasoner is executed to report the status of the ontology, i.e., whether the ontology is consistent, inconsistent or has unsatisfiable classes. The addition of the auxiliary axioms needs to always lead to a consistent ontology. However, in the case of the assertions, the agreement between the reasoner status after the addition of all the axioms and the status indicated in the test implementation determines whether the ontology satisfies the desired behaviour and, consequently, the requirement. These steps carried out during the execution activity are summarised in Algorithm 1 .

During this activity, the test implementations should be first completed with the information related to the ontology in order to be executed. To that end, a glossary of terms must be generated manually or automatically. As mentioned before, the glossary of terms maps each term in the test with a term in the ontology to be analysed. Therefore, the terms that are defined in the ontology, e.g., Gateway, are collected and associated with a URI in the ontology, e.g., «http://www.example.org/ontology/ns\#Gateway». Then, using these associations, the terms in the test implementation are translated into terms in the ontology. Consequently, these test implementations can then be executed 
on the ontology. This requires that the terms in the test expressions must be included in the glossary. However, it is possible that a term in the test expression is not included in the glossary of terms of the ontology. In this case, the ontology does not include the terms asked by the test and, therefore, the test is not passed.

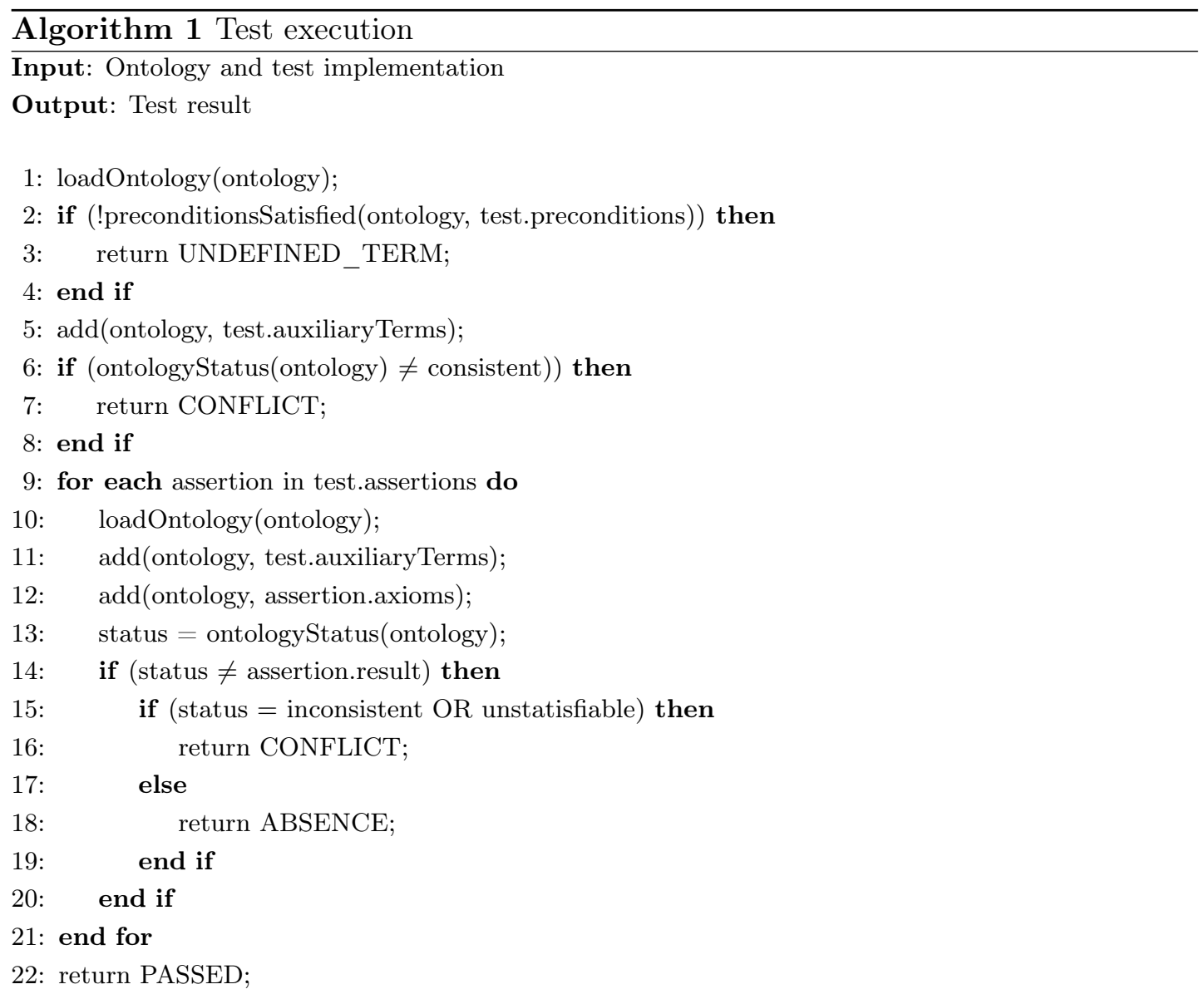

There are four possible results of the execution step for each test and each ontology:

1. Passed: if the ontology passes the preconditions and the results of the assertions are the expected ones.

2. Undefined term: if the ontology does not pass the preconditions, i.e., some of the terms in the test expression are not defined in the ontology. 
3. Absent relation: if the ontology passes the preconditions and the results of the assertion are not the expected ones but there are no conflicts in the ontology.

4. Conflict: if the ontology passes the preconditions and the results of the assertion are not the expected ones, and the addition of the axioms related to the test expression leads to a conflict in the ontology.

\subsection{Verification Test Case ontology}

An ontology for modelling tests could provide not only a guide on how to create test for ontology verification, but also a procedure for creating reusable tests and for allowing traceability between tests, requirements and ontologies. Moreover, the test suites written following the ontology with all the information related to the requirements and the tests can be considered as formalised documentation. For this reason, the Test Case Verification ontology ${ }^{30}$ has been developed. This ontology, which is shown in Figure 4.3 , includes the following information:

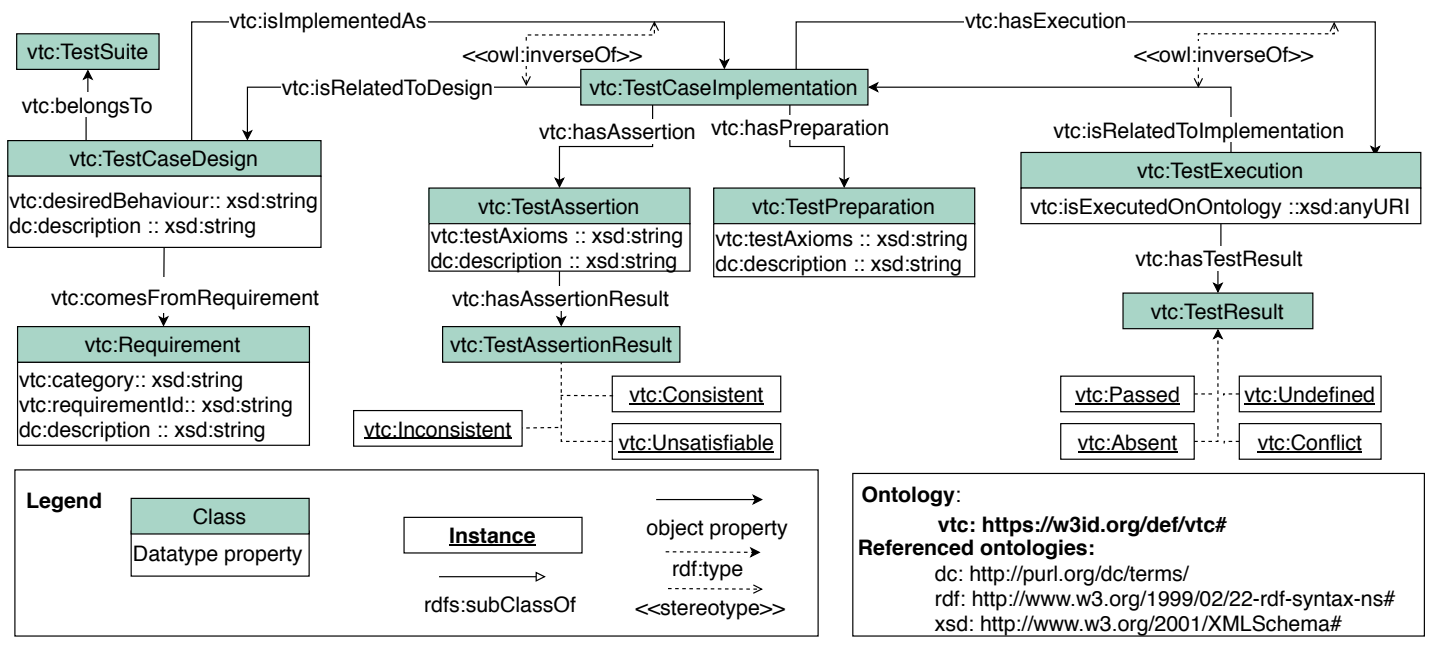

Figure 4.3: Overview of the Test Case Verification ontology

- The vtc:TestCaseDesign class defines the design of a test. It has several datatype properties, including description, desired behaviour (i.e., the test), and related requirements, in order to enable traceability between the test and the requirements from which it is extracted.

\footnotetext{
${ }^{30}$ The Verification Test Case ontology is available online: https://w3id.org/def/vtc
} 
- A vtc:TestCaseDesign belongs to a particular vtc:TestSuite, which is extracted from a given source (e.g., a manual, standard, or list of requirements of an ontology). It can be associated with a vtc:Requirement from which it is extracted, which has a category, an identifier and a description.

- The vtc:TestCaselmplementation class defines how each test design should be implemented. Each vtc:TestCaselmplementation is related to a vtc:TestCaseDesign. A vtc:TestCaselmplementation includes a vtc:TestPreparation, which represents the temporary axioms added to declare the auxiliary terms, and a vtc:TestAssertion, which represents the axioms to be added to represent each ontology scenario. Each vtc:TestAssertion has a vtc:AssertionResult, which is represented by means of three individuals, namely (1) vtc:Unsatisfiable, (2) vtc:Inconsistent and (3) vtc:Consistent.

- The vtc:TestExecution class defines the execution of a test implementation on a particular ontology. Therefore, it is related to a vtc:TestCaselmplementation and an ontology. A vtc:TestCaseResult has also associated a vtc:TestResult, which represents the result of the test execution. The result is represented by means of four individuals, namely (1) vtc:Passed, (2) vtc:Undefined, (3) vtc:Absent and (4) vtc:Conflict.

Listing 4.1 presents a short illustrative example of a test design, generated from the requirement which states "An IoT gateway is a digital entity", which is categorised with one requirement type: "T2. Subsumption relation between classes A and B". Because of the fact that this test does not have URIs related to the ontology in which the tests are going to be executed, it can be reused in other ontologies.

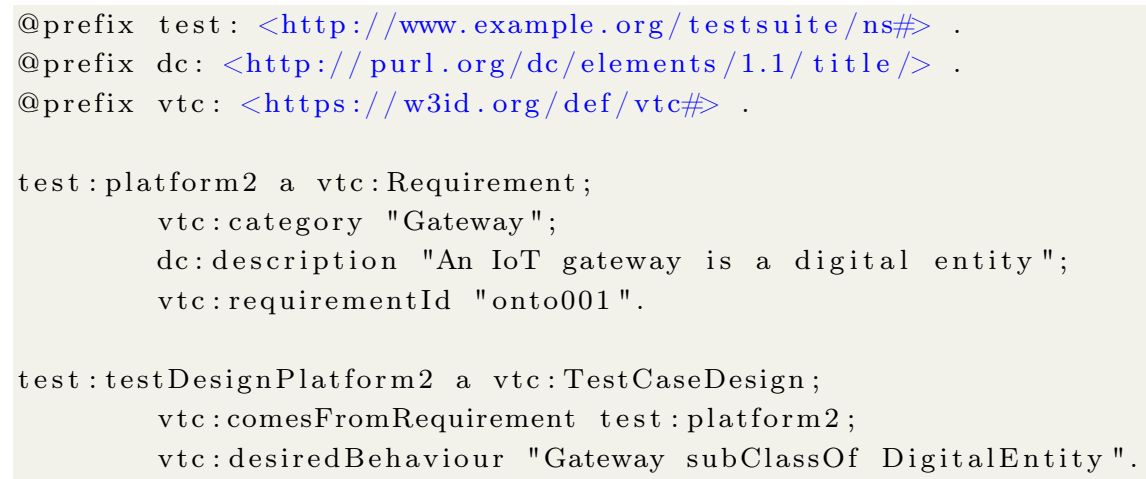

Listing 4.1: Example of test design 
Listing 4.2 presents the test implementation for the test case design presented in Listing 4.1. The figure illustrates the test precondition, which verifies that the classes involved in the test must exist in the ontology; the test preparation, which adds the auxiliary terms needed for the execution of the test; and the assertions.

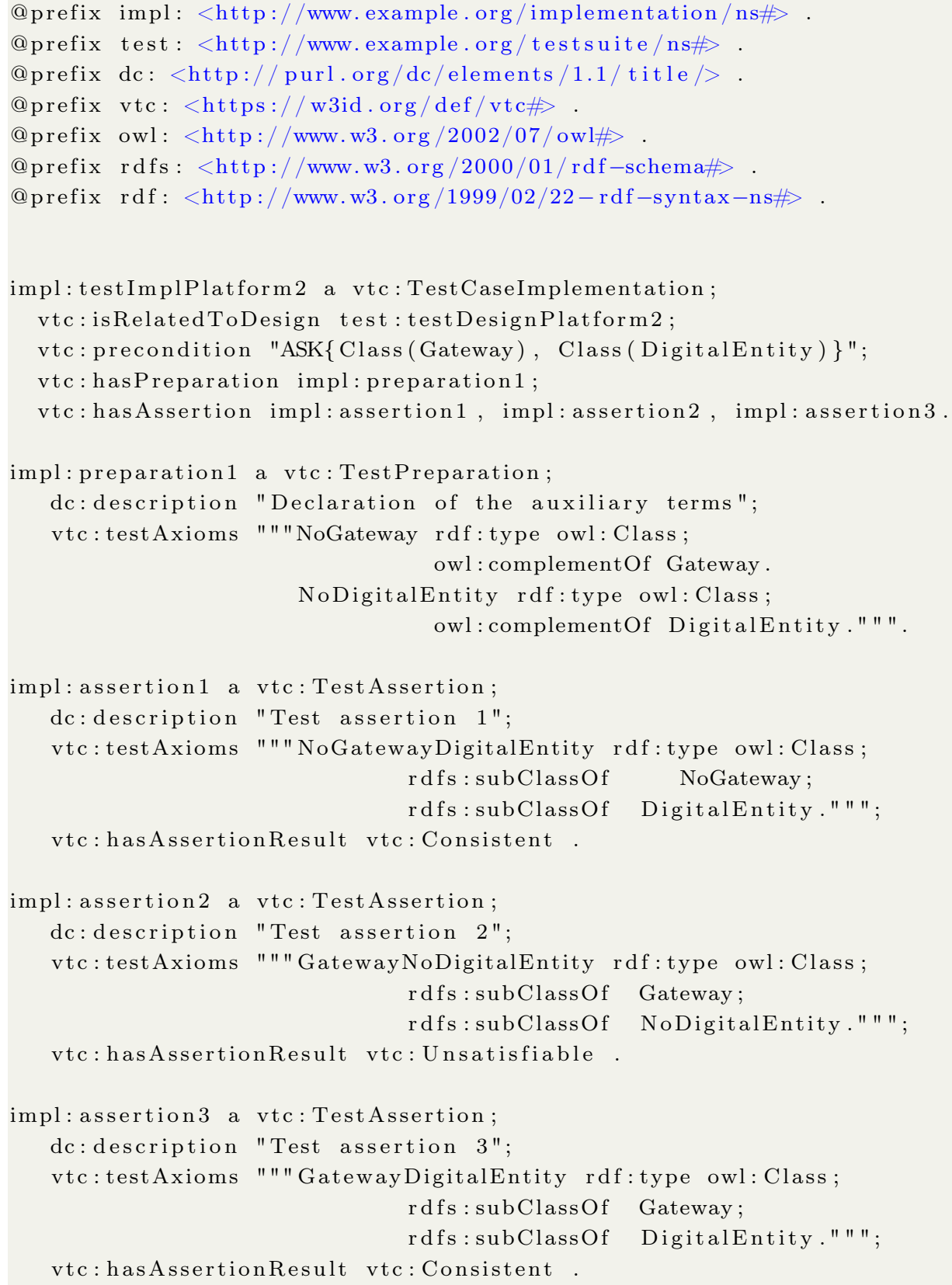

Listing 4.2: Example of test implementation 
Listing 4.3 presents an example of test result associated with the test implementation presented in Listing 4.2. Such implementation was executed on the ontology with URI «http://www.example.org/ontology/ns\#». The test execution states that the test implementation executed in such ontology results in passed, that means, the test is passed by the ontology.

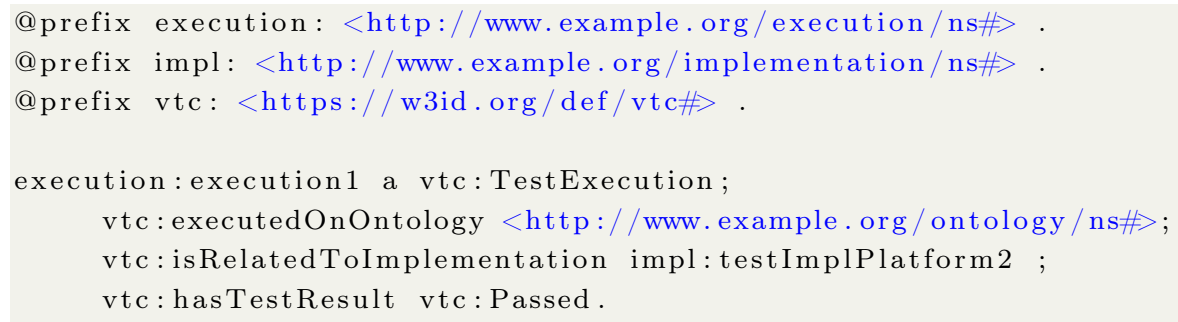

Listing 4.3: Example of test result

\subsection{Technological support for ontology testing: Themis}

Verifying an ontology manually can be a tedious and time-consuming task, and can lead to errors. Therefore, it is advisable to develop technological support that assists developers and practitioners while they are verifying an ontology.

This section presents Themis 31 an on-line testing tool intended to help ontology engineers and practitioners during the ontology verification activity. Themis supports and automates the test implementation and execution steps defined in Section 4.2. It provides both a web-based human interface and a REST API to be used by applications. It is worth noting that the repair activity is out of scope of Themis at the moment of writing this dissertation. Themis is implemented in Java and uses well-known opensource frameworks and libraries. The code of the tool is available in Github 32 under Apache License 2.0 33

\subsubsection{System architecture}

Themis was developed as an online tool to be suitable for a broader range of users as no previous installation is needed. This decision allows us to keep an updated version of the tool, instead of having different releases.

\footnotetext{
${ }^{31}$ http://themis.linkeddata.es

32 https://github.com/oeg-upm/Themis

33 https://www .apache.org/licenses/LICENSE-2.0
} 
Figure 4.4 presents the underlying architecture of Themis. This architecture is based on three layers, namely, the presentation, the business and the persistence layers.



Figure 4.4: Themis architecture overview

The presentation layer allows the interaction with the system for the users. It is accessible by means of the web user interface and the web REST API. The users should enter the ontology to be tested by its URI or its OWL implementation, as well as the test expression or set of test expressions needed to verify the ontology. In the web interface, the tests can be entered directly through the web user interface, or by means of uploading an RDF file with the test suite following the Verification Test Case ontology. Themis includes a syntax checker that indicates in red when the test is not syntactically correct, and an autocomplete service to guide the user in the definition of the tests.

Once the ontology is loaded, Themis generates automatically a glossary of terms, which is used to complete the test implementation for the loaded ontology. This glossary also indicates which terms are classes, properties or individuals, and can be up- 
dated by users through the interface if needed. The terms included in the glossary are the fragments of the URIs of each concept in the loaded ontology, e.g., from the URI «http://www.example.org/ontology/ns\#Gateway» the term Gateway will be extracted as a keyword in the glossary.

Themis graphical user interface also allows users to export the tests that have been added to the interface in an RDF file following the Verification Test Case ontology presented in Section 4.3 .

The business layer is in charge of producing the test results. For doing so, it takes as input the ontology to be tested and the set of tests. The ontology is then loaded into the system using an RDF parser. Afterwards, the system takes the tests and implements them as described in Section 4.2.2. Themis supports the implementation of the 28 test expressions described in Section 4.2.1.

Once the test expressions are executed on the ontology, the following information is provided: (1) the ontology in which the test has been executed; (2) the executed test; (3) the type of the result; and (4) the problem encountered, if any. The results of Themis are aligned with the results described in the test execution activity in Section 4.2 .3 .

Finally, the persistence layer keeps track of the test expressions introduced in the tool, as well as the glossary of terms.

\subsubsection{Themis REST API}

While the main goal was to provide ontology engineers and practitioners with a graphical user interface for testing their ontologies, it is useful to allow third-party software to run testing processes too.

As mentioned in the system architecture, Themis is also available as a REST API 34 Such API allows third-party software to integrate Themis features providing the following functionalities:

- To execute tests on an ontology or set of ontologies. The API can execute tests from ontology URIs or ontology codes.

- To autocomplete the test. It returns the next term or terms that can be added to the incomplete test provided as input.

- To check the test syntax. It validates whether the syntax of the tests is correct.

\footnotetext{
${ }^{34}$ The documentation of the API is available online: http://themis.linkeddata.es/ swagger-ui/index.html
} 
- To generate the glossary of terms of an ontology. The API can generate the glossaries from ontology URIs or ontology codes.

- To generate an example file. It generates a file with several example tests based on an ontology URI.

\subsubsection{Dependencies}

In the implementation of Themis, Maven is used for project and dependencies management.

Additionally, Themis uses the OWL API ${ }^{35}$ which is an open-source Java API and reference implementation for creating, manipulating and serialising OWL ontologies. The OWL API includes parsers and writers for several OWL serialisations, such as RDF/XML, OWL/XML and Turtle. In addition, it also includes interfaces for working with several reasoners. The OWL API is licensed under dual license mode: LGPL and Apache.

Themis also uses Jersey, an open source framework 36 for developing RESTful Web Services in Java. It is licensed under the Eclipse Public License 2.0 ${ }^{37}$

Finally, Themis uses Pellet ${ }^{38}$ an open-source Java-based OWL 2 reasoner. It is an OWL-DL reasoner with support for reasoning with individuals, user-defined datatypes, and debugging support for ontologies.

\footnotetext{
$\sqrt[35]{\text { http://owlapi.sourceforge.net }}$

36 https://eclipse-ee4j.github.io/jersey/

37 https://www.eclipse.org/legal/epl-2.0/

38 http://pellet.owldl.com
} 


\section{Chapter 5}

\section{Ontology metrics derived from the ontology verification process}

Software metrics play an important role in the Software Engineering field, supporting both development and managerial decision-making during the software life-cycle. These software metrics are not only related to the source code itself, but also to other artefacts that are part of the software main product, e.g., requirements and tests, and to the activities needed to obtain such artefacts. This diversity of metrics enables software engineers to have enough information to make different types of predictions, assessments and trade-offs (Fenton and Neil, 2000).

In fact, in real-world projects it is necessary to be aware of the status of the product at every step of the development process, in order to be able to plan and manage its development in an efficient way. Thus, metrics should provide valuable information about the testing artefacts generated during the development process, such as those related to requirements and tests, with the aim of monitoring the product through its life-cycle, being aware of whether the product satisfies all the expected requirements or being aware of the quality of such requirements.

In the Ontology Engineering field multiple metrics exist which try to assess the quality of ontologies by measuring reliability, reusability, or cohesion, among other aspects. However, current metrics are focused on the ontology implementation, leaving aside other artefacts such as the verification ones. Moreover, they usually consider the structure of the ontology (Vrandečić and Sure, 2007).

This chapter focuses on describing ontology metrics related to different artefacts generated and used during the ontology verification process, more specifically, artefacts related to the requirements, the tests and the ontology implementation. To that end, 
different roles involved in the ontology development process, their needs and their risks regarding ontology metrics have been identified. The identified metrics allow those roles to gather information about the their needs, e.g., about the specification of the requirements or the results of their ontologies during the verification activity. First, Section 5.1 describes the methodology followed to identify the metrics, while the description of the roles in Ontology Engineering is presented in Section 5.2 and the description of the considered artefacts is presented in Section 5.3. Finally, Section 5.4 describes the proposed ontology engineering metrics.

\subsection{Ontology metrics identification}

In order to identify the set of metrics that allow monitoring the artefacts generated and used during the verification process several steps were followed. Along this section, the workflow followed to identify and classify metrics is described. As shown in Figure 5.1 . the steps carried out during the workflow were the following:

1. Roles identification: The different roles involved in the ontology development process, their needs from a verification perspective and their risks regarding ontology metrics were identified.

2. Artefacts identification: The artefacts generated during the ontology development process that could be potentially useful in order to support the abovementioned needs and risks were identified. These artefacts refer to the ontology implementation, the ontology requirements specification document and the ontology tests. They are the artefacts needed in the verification testing method presented in Chapter 4

3. Existing ontology metrics analysis: In order to carry out this step, the current state of the art in ontology metrics was analysed, leading to the identification of already existing ontology metrics that could be useful to support the identified needs.

4. Ontology metrics proposal: The set of new metrics proposed was developed taking into account: a) the existing ontology metrics in the literature; b) the needs of the different actors involved in the ontology development process; and c) the artefacts generated during the ontology development process. These metrics were also classified according to whether they are product or process metrics. In the case of product metrics, they were also classified according to whether they are base or calculated metrics. 


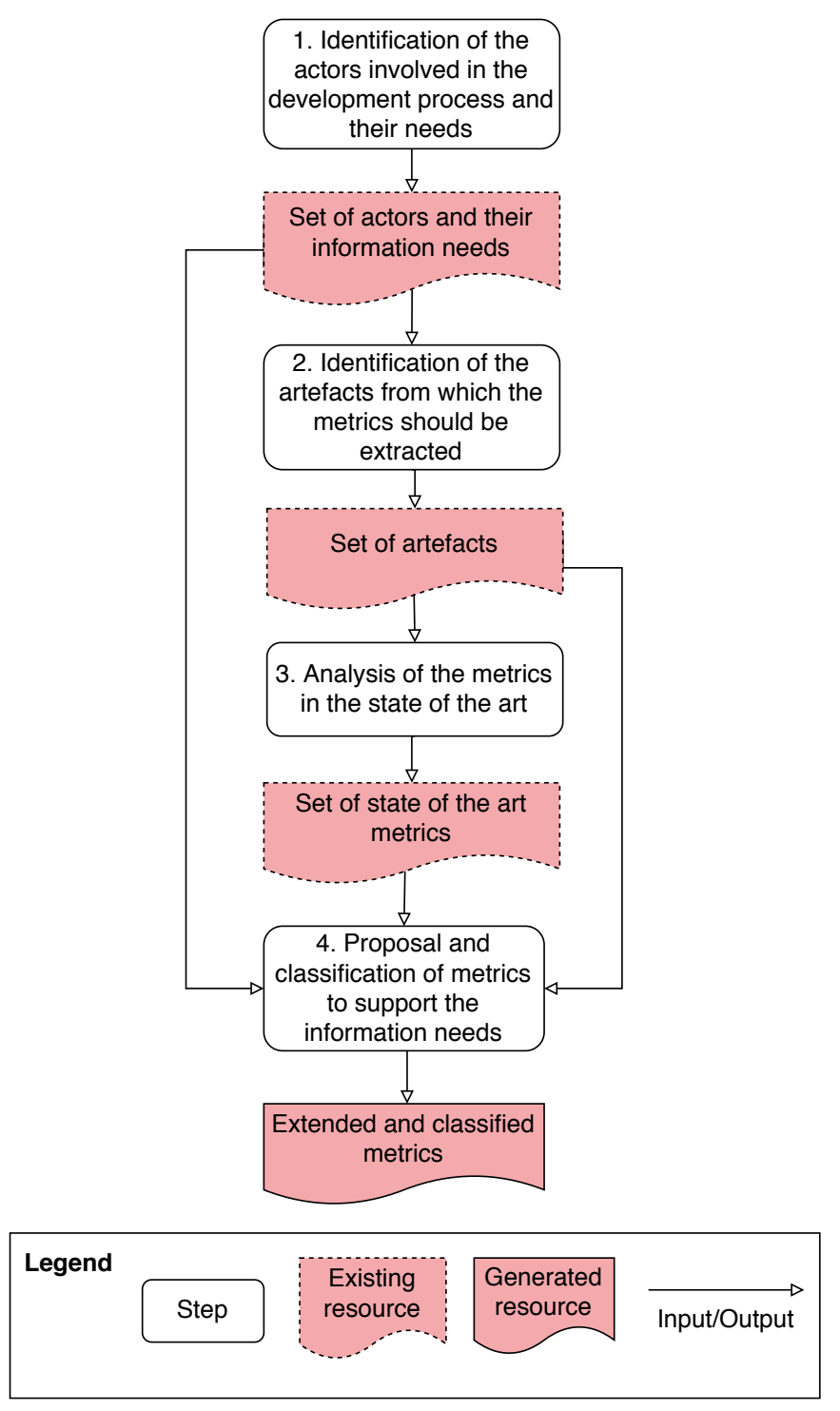

Figure 5.1: Activity workflow followed to generate the proposed set of metrics

\subsection{Roles in Ontology Engineering}

In order to define the set of ontology engineering metrics, the roles that should be involved in the ontology development process were identified along with what they want to monitor. Ontologies are being increasingly adopted in software projects and, therefore, the application of software practices in Ontology Engineering can be useful for easing this integration. Accordingly, this identification of actors in Ontology Engineering was based on the Software Engineering state of the art (Pressman, 2005) applied to 
ontology engineering practices. As a result, the following roles were identified:

- Team leaders. People in charge of monitoring and managing the development process and of diagnosing technical and organisational issues. These actors have the following needs:

- To organise the development tasks.

- To detect issues that can hinder the development process.

- Ontology engineers. People responsible for creating the ontologies and all the resources needed to publish and document them. These actors have the following needs:

- To be aware of whether the ontology they are generating is correct.

- To be aware of whether the ontology they are generating is complete.

- Stakeholders. Customers, users and domain experts who specify the requirements for the ontology to be created. These actors have the following needs:

- To be aware of whether the ontology they are generating is correct.

- To be aware of whether the ontology they are generating is complete.

- To be aware of whether all the requirements they propose are satisfied by the ontology.

- If they are customers, they need to be aware of the status of the ontology development process.

Also inspired by Software Engineering, the risks that can be monitored using metrics during the development process were taken into account, such as those mentioned by Pressman (Pressman, 2005), namely: (1) Performance risk, i.e., the degree of uncertainty that the product will meet its requirements and be fit for its intended use; or (2) Schedule risk, i.e., the degree of uncertainty that the project schedule will be maintained and that the product will be delivered on time. The former risk should be monitored by the ontology engineers, while the latter one should be monitored by the team leaders. 


\subsection{Development artefacts in the ontology verification pro- cess}

In order to propose metrics for Ontology Engineering related to the verification process with the goal of monitoring the development process, three artefacts are considered to calculate such metrics, namely:

- The ontology requirements specification document (ORSD), which is the product resultant from the ontology requirements specification activity (SuárezFigueroa et al., 2015). In this document the purpose, requirements and users of the ontology are identified.

- The ontology requirements test suite (ORTS), which is the product generated during the verification testing process, such as the set of tests resultant from the verification testing method described in Chapter 4

- The ontology implementation, which is the product resultant from the ontology implementation activity.

These artefacts can be generated iteratively due to the fact that, following ontology development methodologies (e.g., eXtreme design (Presutti et al., 2009), SAMOD (Peroni, 2016), NeOn (Suárez-Figueroa et al., 2015), GOSPL (Debruyne et al., 2013) and UPON (De Nicola et al. 2005)), there can be several versions of an ontology implementation.

Several tools for ontology development can be used to manage these artefacts, including their generation and maintenance. These tools include not only ontology editors, such as Protégé, but also platforms for storing and versioning resources, such as GitHub ${ }^{39}$ and for editing and sharing requirements specification documents, such as online spreadsheets. In the following sections each of these artefacts is further described.

\subsubsection{Ontology requirements specification document}

The ontology requirements specification document (ORSD) (Suárez-Figueroa et al. 2009b) is built to define the purpose of the ontology, the intended users and the requirements that the ontology should be able to fulfil (Suárez-Figueroa et al., 2015). It is usually generated before the implementation of the ontology during the ontology requirements specification activity. This document should include information related to

\footnotetext{
$\sqrt[39]{\text { https://github.com }}$
} 
the requirement identifier and the statement or competency question associated, which is a natural language sentence that expresses a question people expect the ontology to answer. The ORSD could also include, in order to obtain more metrics, the status of the requirements (e.g., rejected, deprecated or accepted). In case that the ontology development process follows an iterative approach, it could also include the development iteration or sprint in which the requirement is planned to be implemented.

\subsubsection{Ontology requirements test suite}

An ontology requirements test suite (ORTS) is a collection of tests extracted from ontology requirements that are intended to be used to test an ontology. Since this chapter is focused on metrics from the ontology verification activity, the tests stored in the test suite should include the requirements translated into a formal language (e.g., using the testing language presented in Chapter 4 and the identifier of the associated requirement in the ORSD. This test suite could be stored in RDF following the Verification Test Case ontology presented in Section 4.3, which allows storing all the information related to the tests, as well as the associated requirement and ontology implementation. Moreover, the association between tests, requirements and ontology in the test suite enables the traceability between these resources.

This artefact can be generated before the implementation of the ontology in order to follow a test-driven development approach (Keet and Ławrynowicz, 2016), or after the implementation of the ontology in order to follow a cascade development approach (Fernández-López et al., 1997).

\subsubsection{Ontology implementation}

The main artefact from which ontology metrics can be extracted is the ontology implementation itself, which is the most common artefact used to define metrics in Ontology Engineering. The ontology implementation contains the axioms and metadata of the ontology and should be encoded through a formal language like RDF Schema (Brickley and Guha, 2014) or OWL (Hitzler et al., 2012).

\subsection{Ontology Engineering metrics}

Inspired by the Software Engineering classification of metrics (Sommerville, 2010), the ontology metrics presented in this section are divided into two categories, i.e., product metrics and process metrics. In the Ontology Engineering context, product metrics 
are used to measure different internal attributes of the resultant ontologies, such as size or complexity of the ontology implementation. Besides, process metrics are used to measure different attributes of the ontology development process, such as time, changes or resources, and enable ontology engineers to assess project status, track potential risks, adjust workflows and predict personnel effort.

Additionally, the set of product and process metrics are related to each of the artefacts described in Section 5.3, i.e., the ORSD, the ORTS and the ontology implementation. The product metrics are related to a concrete version of these artefacts, while the process metrics are related to two or more versions. In the case that more than one of the mentioned artefacts are available, it is possible to combine the data extracted from them by generating multi-artefact metrics, which are also defined in this section.

To define these metrics, several principles were also followed. First, the metrics presented in this chapter reuse existing metric definitions. In such cases, the reference to the existing bibliographic resource is shown along with the metric. In addition, these metrics are related to artefacts that are not analysed in the state of the art so far, i.e., the ORSD and the ORTS. Finally, the data extracted from these artefacts was combined to extract measurements with the aim of covering several aspects in the ontology development process, e.g., the analysis of the requirements coverage or the effort needed to develop an ontology. Therefore, this section describes metrics based on the needs of the actors identified in Section 5.2 and on the integration of data from several of the artefacts presented in Section 5.3 rather than to provide an exhaustive analysis about all the feasible ontology metrics that can be extracted from them. Figure 5.2 summarises the set of metrics and the artefacts from which they are extracted, and also shows potential interests of each actor and metric.

The following subsections describe the proposed metrics. The metrics are organised into tables which include four fields, namely: (a) the artefact or artefacts from which each metric is extracted, (b) the code to identify the metric, (c) the name and definition of each metric, and (d) the formula needed to calculate it. In order to ease readability, product metrics are grouped into two categories, i.e., base metrics (Table 5.1), which represent raw data, and calculated metrics (Table 5.2), which are derived from base metrics. Finally, Table 5.3 shows the process metrics that are related to the process life cycle.

\subsubsection{Base product metrics}

Table 5.1 describes the proposed set of base product metrics related to the ORSD, the ORTS and the ontology implementation. It shows by means of the symbol " $\checkmark$ " which 
artefact or artefacts are needed to calculate each metric. These metrics are going to be used to obtain calculated metrics, which are described in the next subsection. These base metrics can be extracted from one or more of the identified artefacts.

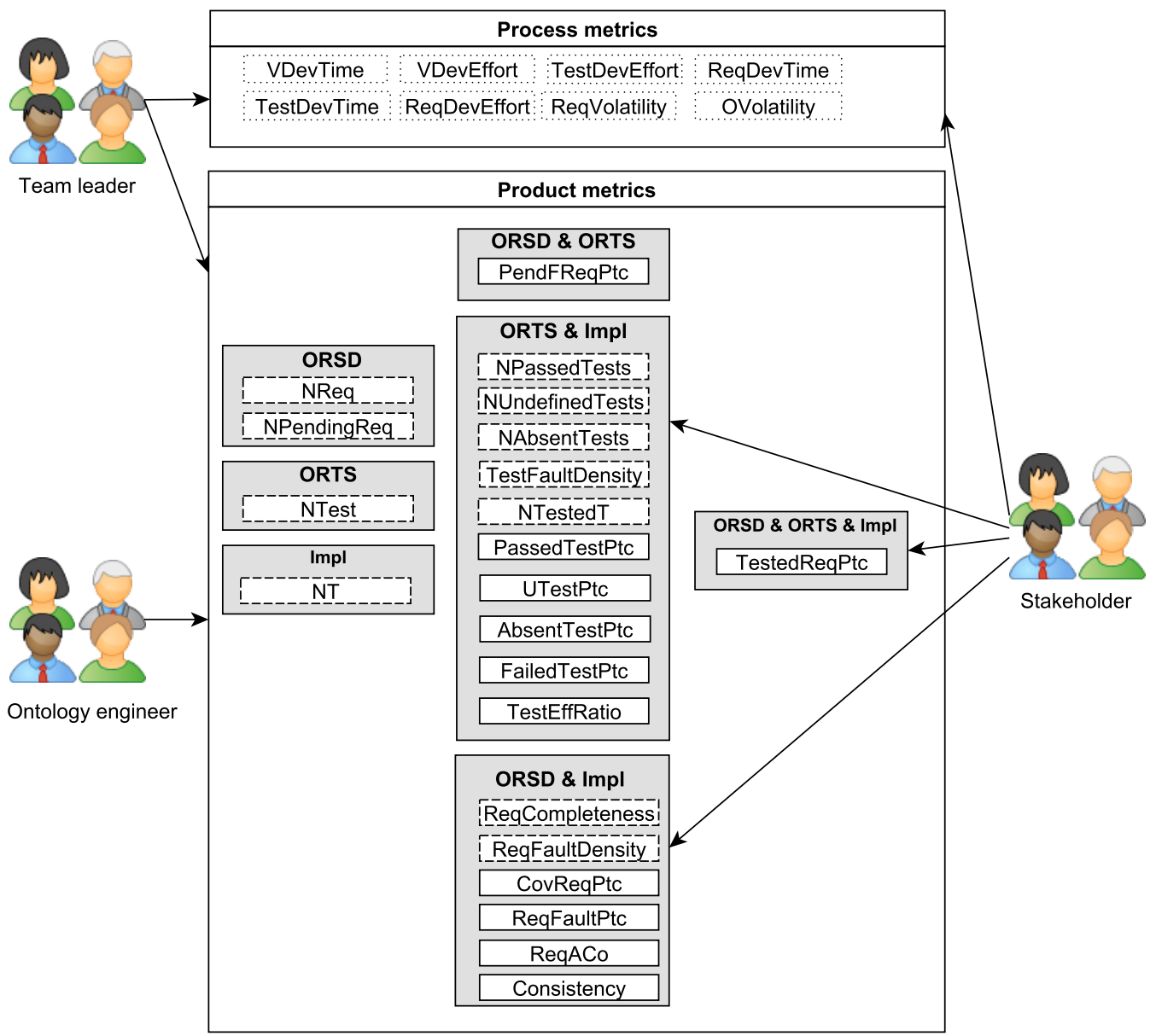

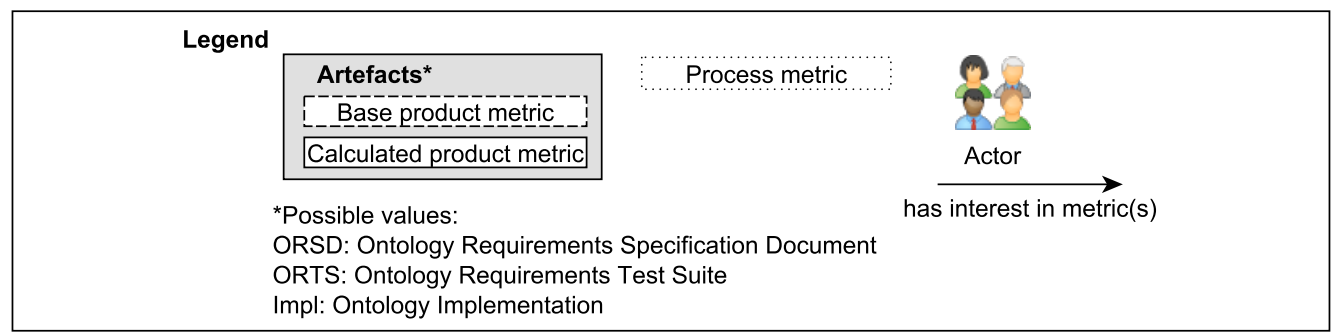

Figure 5.2: Ontology engineering metrics and interested actors. 
Table 5.1: Base product metrics and artefacts from which they are extracted

\begin{tabular}{|c|c|c|c|c|c|}
\hline \multicolumn{3}{|c|}{ Artefacts } & \multirow[b]{2}{*}{ Code } & \multirow[b]{2}{*}{ Name and definition } & \multirow[b]{2}{*}{ How to calculate } \\
\hline 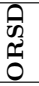 & $\begin{array}{l}\omega_{2} \\
\hat{\sim}_{1}^{2} \\
0\end{array}$ & 官 & & & \\
\hline$\checkmark$ & & & NReq & $\begin{array}{l}\text { Number of Requirements. Number of Require- } \\
\text { ments defined in the ORSD. }\end{array}$ & $\begin{array}{l}\text { Obtained by counting the number of require- } \\
\text { ments in a given ORSD. }\end{array}$ \\
\hline & & & NPendReq & $\begin{array}{l}\text { Number of Pending Requirements. Number of } \\
\text { pending to implement requirements after a de- } \\
\text { velopment iteration. }\end{array}$ & $\begin{array}{l}\text { Obtained by counting the number of pending } \\
\text { requirements in a given ORSD. }\end{array}$ \\
\hline & & $\checkmark$ & NT & $\begin{array}{l}\text { Number of vocabulary Terms. Number of } \\
\text { terms that are included in a particular im- } \\
\text { plementation of an ontology. To increase its } \\
\text { granularity, this metric can be subdivided into } \\
\text { Number of classes (Zhe et al., 2006) and Num- } \\
\text { ber of properties (Zhe et al., 2006). }\end{array}$ & $\begin{array}{l}\text { Obtained by counting terms (i.e., classes and } \\
\text { properties) in an ontology implementation. }\end{array}$ \\
\hline & $\checkmark$ & & NTests & $\begin{array}{l}\text { Number of Tests. Number of tests defined } \\
\text { based on the ORSD. }\end{array}$ & $\begin{array}{l}\text { Obtained by counting tests in a given ontology } \\
\text { requirements test suite. }\end{array}$ \\
\hline & $\checkmark$ & $\checkmark$ & NPassedTests & $\begin{array}{l}\text { Number of Passed Tests. Number of tests in } \\
\text { the test suite which are passed by the ontology } \\
\text { implementation. }\end{array}$ & $\begin{array}{l}\text { Obtained by counting the number of tests that } \\
\text { are passed by a given ontology implementa- } \\
\text { tion. }\end{array}$ \\
\hline & $\checkmark$ & $\checkmark$ & NUndefinedTests & $\begin{array}{l}\text { Number of Undefined Tests. Number of tests } \\
\text { in the test suite which result in "undefined } \\
\text { terms"a by the ontology implementation. }\end{array}$ & $\begin{array}{l}\text { Obtained by counting the number of tests that } \\
\text { result in "undefined terms" by a given ontology } \\
\text { implementation. }\end{array}$ \\
\hline & $\checkmark$ & $\checkmark$ & NAbsentTests & $\begin{array}{l}\text { Number of Absent Tests. Number of tests in } \\
\text { the test suite which result in "absent"a by the } \\
\text { ontology implementation. }\end{array}$ & $\begin{array}{l}\text { Obtained by counting the number of tests that } \\
\text { result in "absent" by a given ontology imple- } \\
\text { mentation. }\end{array}$ \\
\hline & $\checkmark$ & $\checkmark$ & TestFaultDensity & $\begin{array}{l}\text { Tests Fault Density. Number of tests in the } \\
\text { test suite that are not passed by the ontol- } \\
\text { ogy implementation. Inspired by Costello and } \\
\text { Liu, 1995. }\end{array}$ & $\begin{array}{l}\text { Obtained by counting the number of tests that } \\
\text { are not passed by a given ontology implemen- } \\
\text { tation. }\end{array}$ \\
\hline & $\checkmark$ & $\checkmark$ & NTestedT & $\begin{array}{l}\text { Number of Tested vocabulary Terms. To in- } \\
\text { crease its granularity, this metric can be subdi- } \\
\text { vided into Number of tested classes and Num- } \\
\text { ber of tested properties. }\end{array}$ & $\begin{array}{l}\text { Obtained by counting unduplicated terms (i.e., } \\
\text { classes and properties) in a given ontology test } \\
\text { suite. }\end{array}$ \\
\hline$\checkmark$ & & $\checkmark$ & ReqCompleteness & $\begin{array}{l}\text { Requirements Completeness. Number of re- } \\
\text { quirements covered by the ontology. Inspired } \\
\text { by Costello and Liu, 1995. }\end{array}$ & $\begin{array}{l}\text { Obtained by counting the number of require- } \\
\text { ments that are covered by a given ontology im- } \\
\text { plementation. }\end{array}$ \\
\hline$\checkmark$ & & $\checkmark$ & ReqFaultDensity & $\begin{array}{l}\text { Requirements Fault Density. Number of re- } \\
\text { quirements faults. Inspired by Costello and } \\
\text { Liu, 1995. }\end{array}$ & $\begin{array}{l}\text { Obtained by counting the number of require- } \\
\text { ments faults in an ORSD. }\end{array}$ \\
\hline
\end{tabular}

a This result is explained in Section 4.2 .3 since it is a potential result from the verification testing activity described in Chapter 4. 


\subsubsection{Calculated product metrics}

Table 5.2 describes the proposed set of calculated product metrics related to the three artefacts, i.e., the ORSD, the ORTS and the ontology implementation. This table shows by means of the symbol " $\checkmark$ " which artefacts are needed to calculate each metric. These metrics are calculated using several base metrics, which are described in the previous subsection, and are extracted from more than one of the identified artefacts. As an example of a calculated metric, the metric CovReqPtc is obtained by using the base metrics NCovReq and NReq, and is extracted from the ORSD and the ontology implementation artefacts.

Table 5.2: Calculated product metrics and artefacts from which they are extracted

\begin{tabular}{|c|c|c|c|c|c|}
\hline \multicolumn{3}{|c|}{ Artefacts } & \multirow[b]{2}{*}{ Code } & \multirow[b]{2}{*}{ Name and definition } & \multirow[b]{2}{*}{ How to calculate } \\
\hline $\begin{array}{l}0 \\
\text { जी } \\
\text { జ̂. } \\
0 \\
0\end{array}$ & 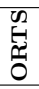 & $\dot{\vec{a}}$ & & & \\
\hline$\checkmark$ & & $\checkmark$ & CovReqPtc & $\begin{array}{l}\text { Covered Requirements } \\
\text { Percentage. Percentage of } \\
\text { requirements covered by } \\
\text { the ontology. }\end{array}$ & $\begin{array}{l}\operatorname{Cov} \operatorname{Req} P t c(\operatorname{Req}, O)=\frac{N \operatorname{Cov} \operatorname{Req}(\operatorname{Req}, O)}{N \operatorname{Req}(\operatorname{Req})} \times 100 \\
\text { where } O \text { represents the ontology implementation to be analysed } \\
\text { and } \operatorname{Req} \text { the ORSD. }\end{array}$ \\
\hline$\checkmark$ & & $\checkmark$ & ReqFaultPtc & $\begin{array}{l}\text { Requirements Fault Per- } \\
\text { centage. Percentage of re- } \\
\text { quirements faults. }\end{array}$ & $\begin{array}{l}\operatorname{ReqFaultPtc}(\operatorname{Req}, O)=\frac{\operatorname{ReqFaultDensity}(\operatorname{Req}, O)}{N \operatorname{Req}(\operatorname{Req})} \times 100 \\
\text { where } O \text { represents the ontology implementation to be analysed } \\
\text { and } \operatorname{Req} \text { the ORSD. }\end{array}$ \\
\hline$\checkmark$ & & $\checkmark$ & ReqACo & $\begin{array}{l}\text { Requirements Axiom Com- } \\
\text { plexity. Percentage of } \\
\text { OWL, RDF and RDFS } \\
\text { constructs related to each } \\
\text { requirement. }\end{array}$ & $\begin{array}{l}\operatorname{ReqACo}(\operatorname{Req}, O)=\frac{\sum_{k=1}^{n} \text { hasAxiom }\left(\operatorname{Req}, A_{k}(O)\right)}{A(O)} \times 100 \\
\text { where } \\
\text { hasAxiom }\left(\operatorname{Req}, A_{k}(O)\right)= \begin{cases}1 & \text { if } \operatorname{Req} \text { includes } A_{k}(O) \\
0 & \text { if } \operatorname{Req} \text { not includes } A_{k}(O)\end{cases} \\
n \text { is the total number of ontology axioms }{ }^{\mathrm{b}}\end{array}$ \\
\hline$\checkmark$ & & $\checkmark$ & Consistency & $\begin{array}{l}\text { Consistency. Percentage of } \\
\text { requirements in the ORSD } \\
\text { that are in conflict. In- } \\
\text { spired by Davis et al., } \\
\text { 1993). }\end{array}$ & $\begin{array}{l}\text { Consistency }(\operatorname{Req})=\frac{\text { InconsistentReqs }(\operatorname{Req})}{N \operatorname{Req}(\operatorname{Req})} \times 100 \\
\text { where } O \text { represents the ontology implementation to be analysed } \\
\text { and } \operatorname{Req} \text { the ORSD. }\end{array}$ \\
\hline$\checkmark$ & $\checkmark$ & & PendFReqPtc & $\begin{array}{l}\text { Pending Formalised Re- } \\
\text { quirements Percentage. } \\
\text { Percentage of pending } \\
\text { requirements which are } \\
\text { already included in the } \\
\text { test suite. }\end{array}$ & 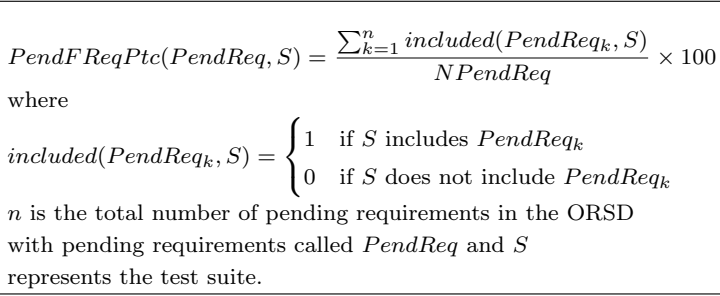 \\
\hline
\end{tabular}

a The axiom considered from RDF in this metric is: type. The axioms considered from RDFS are: subClassOf, subPropertyOf, domain and range. The axioms considered from OWL are: cardinality, complementOf, disjointWith, equivalentClass, equivalentProperty, FunctionalProperty, hasValue, intersectionOf, InverseFunctionalProperty, inverseOf, maxCardinality, minCardinality, oneOf, someValuesFrom, SymmetricProperty, TransitiveProperty and unionOf. However, this can change depending on the needs of the user. 
Table 5.2 - Continued from previous page

\begin{tabular}{|c|c|c|c|c|c|}
\hline \multicolumn{3}{|c|}{ Artefacts } & \multirow[b]{2}{*}{ Code } & \multirow[b]{2}{*}{ Name and definition } & \multirow[b]{2}{*}{ How to calculate } \\
\hline 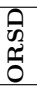 & 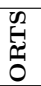 & $\dot{\vec{a}}$ & & & \\
\hline & $\checkmark$ & $\checkmark$ & PassedTestPtc & $\begin{array}{l}\text { Passed Test Percentage. } \\
\text { Percentage of tests passed } \\
\text { by the ontology implemen- } \\
\text { tation. }\end{array}$ & $\begin{array}{l}\text { PassedTestPtc }(S, O)=\frac{N \text { PassedTest }(S, O)}{N \operatorname{Nests}(S)} \times 100 \\
\text { where } S \text { represents the test suite which stores the formalised } \\
\text { requirements and } O \text { the ontology implementation. }\end{array}$ \\
\hline & $\checkmark$ & $\checkmark$ & UTestPtc & $\begin{array}{l}\text { Undefined Test Percent- } \\
\text { age. Percentage of tests } \\
\text { that result in "undefined } \\
\text { terms" by the ontology im- } \\
\text { plementation. }\end{array}$ & $\begin{array}{l}U T \operatorname{estPtc}(S, O)=\frac{\text { NUndefinedTest }(S, O)}{N T e s t s(S)} \times 100 \\
\text { where } S \text { represents the test suite which stores the formalised } \\
\text { requirements and } O \text { the ontology implementation. }\end{array}$ \\
\hline & $\checkmark$ & $\checkmark$ & AbsentTestPto & $\begin{array}{l}\text { Absent Test Percentage. } \\
\text { Percentage of tests result } \\
\text { in "absent" by the ontology } \\
\text { implementation. }\end{array}$ & $\begin{array}{l}\text { AbsentTestPtc }(S, O)=\frac{N A b \operatorname{sentTests}(S, O)}{N \operatorname{Nests}(S)} \times 100 \\
\text { where } S \text { represents the test suite which stores the formalised } \\
\text { requirements and } O \text { the ontology implementation. }\end{array}$ \\
\hline & $\checkmark$ & $\checkmark$ & FailedTestPtc & $\begin{array}{l}\text { Failed Test Percentage. } \\
\text { Percentage of tests not } \\
\text { passed by the ontology } \\
\text { implementation. }\end{array}$ & $\begin{array}{l}\text { FailedTestPtc }(S, O)=\frac{\text { TestFaultDensity }(S, O)}{N T e s t s(S)} \times 100 \\
\text { where } S \text { represents the test suite which stores the formalised } \\
\text { requirements and } O \text { the ontology implementation. }\end{array}$ \\
\hline & $\checkmark$ & $\checkmark$ & TestEffRatio & $\begin{array}{l}\text { Test Effectiveness Ratio. } \\
\text { Percentage of the ontology } \\
\text { implementation terms, in- } \\
\text { cluding classes and proper- } \\
\text { ties, which are defined in } \\
\text { the test suite. Inspired } \\
\text { by Fenton and Bieman, } \\
\text { 1997. }\end{array}$ & $\begin{array}{l}\text { TestEffRatio }(S, O)=\frac{N T e s t e d T(S)}{N T(O)} \times 100 \\
\text { where } S \text { represents the test suite which stores the formalised } \\
\text { requirements associated with the ontology implementation } O \text {. }\end{array}$ \\
\hline$\checkmark$ & $\checkmark$ & $\checkmark$ & TestedReqPtc & $\begin{array}{l}\text { Tested Requirements Per- } \\
\text { centage. Percentage of } \\
\text { requirements identified in } \\
\text { the ORSD which are for- } \\
\text { malised in the test suite } \\
\text { and passed by an ontology } \\
\text { implementation version. }\end{array}$ & $\begin{array}{l}\text { TestedReqPtc }(O, S)=\frac{\operatorname{CovReqPtc}(O) \times \operatorname{PassedTestPtc}(O, S)}{100}, \text { where } \\
S \text { represents the test suite and } O \text { the ontology to be analysed. }\end{array}$ \\
\hline
\end{tabular}

\subsubsection{Process metrics}

In addition to the metrics described in the previous subsections, this section also describes a set of process metrics. These process metrics are related to (1) the time taken for a particular process to be completed; (2) the resources required for a particular process; and (3) the number of occurrences of a particular event. Some of these metrics refer to the changes performed on the artefacts during the development process. Therefore, several versions of each artefact can be considered for calculating the metrics.

The proposed process metrics, which are summarised in Table 5.3, are extracted from the software infrastructure which supports the generation and versioning of the mentioned artefacts. Manual calculation of these metrics is unfeasible, since it would require ontology engineers to keep track of the time spent in each activity. 
Table 5.3: Process metrics

\begin{tabular}{|c|c|c|}
\hline Code & Name and definition & How to calculate \\
\hline VDevTime & $\begin{array}{l}\text { Version Development Time. Time taken to } \\
\text { implement a given version of the ontology. } \\
\text { This time includes the time taken to carry out } \\
\text { all the activities in the development process, } \\
\text { e.g., requirements elicitation and evaluation. }\end{array}$ & \multirow{3}{*}{$\begin{array}{l}\text { Obtained by measuring the time of the different activities } \\
\text { while developing a version of the ontology. This time can be } \\
\text { calculated in minutes, hours or days, depending on the } \\
\text { available granularity. }\end{array}$} \\
\hline ReqDevTime & $\begin{array}{l}\text { Requirement Development Time. Implemen- } \\
\text { tation time taken of each individual require- } \\
\text { ment in the ontology. }\end{array}$ & \\
\hline TestDevTime & $\begin{array}{l}\text { Test Development Time. Development time } \\
\text { taken for each test in the test suite. }\end{array}$ & \\
\hline VDevEffort & $\begin{array}{l}\text { Version Development Effort. Effort taken to } \\
\text { implement a given version of the ontology. } \\
\text { This effort includes the time taken to carry out } \\
\text { all the activities in the development process, } \\
\text { e.g., requirements elicitation and evaluation. }\end{array}$ & \multirow{3}{*}{$\begin{array}{l}\text { Obtained by measuring the effort required for the different } \\
\text { activities while developing a version of the ontology. } \\
\text { This effort can be calculated in Persons-Month. }\end{array}$} \\
\hline ReqDevEffort & $\begin{array}{l}\text { Requirement Development Effort. Effort taken } \\
\text { to generate the specification of the require- } \\
\text { ments. }\end{array}$ & \\
\hline TestDevEffort & $\begin{array}{l}\text { Test Development Effort. Effort taken to gen- } \\
\text { erate each test in the test suite. }\end{array}$ & \\
\hline OVolatility & $\begin{array}{l}\text { Ontology volatility. Number of axioms added } \\
\text { or removed during the development process. }\end{array}$ & $\begin{array}{l}\text { Obtained by counting the number of axioms added or removed } \\
\text { between two versions of the ontology implementation. }\end{array}$ \\
\hline ReqVolatility & $\begin{array}{l}\text { Requirements volatility. Number of require- } \\
\text { ments that changed during the development } \\
\text { process. To increase its granularity, this met- } \\
\text { ric can be subdivided into Number of added re- } \\
\text { quirements (NAddedReq), Number of rejected } \\
\text { requirements (NRejectedReq) and Number of } \\
\text { accepted requirements (NAcceptedReq) }\end{array}$ & $\begin{array}{l}\text { Obtained by counting the number of requirements that have } \\
\text { undergone some change between two versions of the ORSD. }\end{array}$ \\
\hline
\end{tabular}




\section{Chapter 6}

\section{Ontology conformance testing}

In the Software Engineering field, conformance testing refers to the techniques to determine up to what extent an implementation of a particular standard conforms to the requirements of that standard (Moseley et al., 2003). Ensuring conformance plays an important role in systems across many domains and serves to ensure that a product, process, computer program or system meets a defined set of standards and thus is reliable and interoperable.

Borrowing the conformance definition and goal from Software Engineering, in Ontology Engineering conformance testing refers to the purpose of determining up to what extent a particular ontology conforms to the specification of a standard. In this context, the standards to be conformed to could be standard ontologies or data models defined in standards supported by standardisation bodies, such as the ISO/IEC norms.

Ontology conformance testing should allow ontology engineers to build ontologies ensuring reliability and interoperability. However, despite the number of testing approaches and tools for ontology validation and verification (Chapter 2), there are no works focused on providing support for conformance testing in the Ontology Engineering field. Therefore, it is advisable to focus on the development of approaches that assist such conformance testing.

Moreover, conformance testing can also be applied with the aim of analysing the overlap between a set of standards in a particular domain. This conformance analysis provides information related to the overlaps and differences among such standards. To that end, this chapter also presents a method, based on the conformance testing one, for identifying the minimum common knowledge between ontologies. Therefore, it enables to determine up to what extent a set of standard ontologies represents the same knowledge and which are their differences. 
This chapter presents the adaptation of the method for ontology verification testing described in Chapter 4 to provide support for ontology conformance testing (Section 6.1) and for common knowledge identification between ontologies (Section 6.2).

\subsection{Method for ontology conformance testing}

Following Software Engineering techniques and the ISO/IEC 9646 (detailed in Chapter 2.2.3), any ontology conformance testing approach should include a testing process for designing, implementing and executing tests associated with a standard. Such tests, which are extracted from the standard specification to check a set of requirements related to selected features, should be abstract in the sense that they must not include any information about the ontology in which the test will be executed, being independent of any ontology and, consequently, reusable. In the context of Ontology Engineering, a separated activity for analysing the results should also be included, in order to determine up to what extent an analysed ontology is conformant to a particular standard.

This section proposes a method for ontology conformance testing based on functional ontology requirements. To that end, it includes five activities, namely, functional ontology requirements extraction, test design, test implementation, test execution and test results analysis. Figure 6.1 illustrates this ontology conformance testing method based on ontology requirements, together with the inputs and outputs of the different activities. This method starts with the extraction of functional ontology requirements from a standard, which are used to define tests purposes (TPs) that describe the objective of testing. To define such TPs, the test expression catalogue, where all the supported tests are listed, is provided. Once the ontology engineers have the test purposes defined, they have to implement them in order to be executed on a particular ontology. A glossary of terms of the ontology which maps each term in the test with each term in the ontology is also needed to execute such tests. Finally, the results are analysed in the test analysis activity.

\subsubsection{Activities within the method for conformance testing}

Since the ontology verification method described in Chapter 4 already covers design, implementation and execution of tests, this method is built on top of it. However, the focus of these two methods are different: while in the ontology verification testing method the main goal is to verify whether an ontology satisfies the expected requirements, in the conformance testing method the main goal is to check up to what extent an ontology satisfies the specification of a standard, allowing the identification of overlaps and 


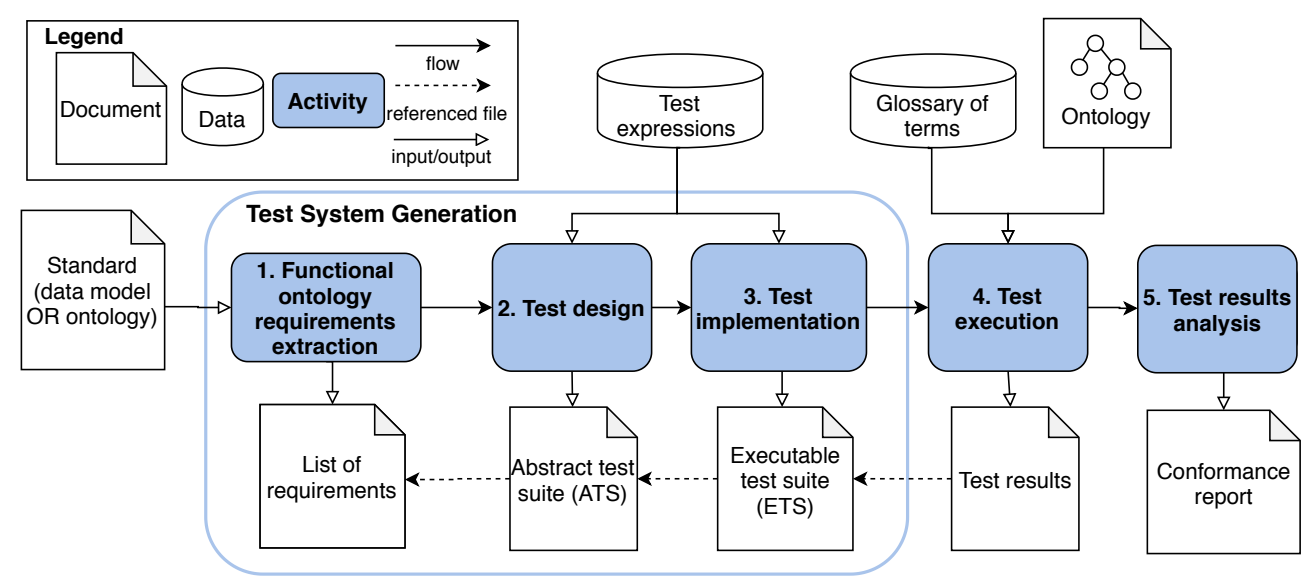

Figure 6.1: Ontology conformance testing approach in ontology engineering

differences between them. When a standard is revised or errata are issued, tests must be updated accordingly and the conformance testing revised. Therefore, although the conformance testing method is based on this ontology testing method, it was adapted and extended in order to support the workflow required in a conformance scenario.

The following sections detail the testing activities that should be carried out in the proposed conformance testing method. The definition of the test design, test implementation and test execution activities are reused from the testing method for ontology verification, which provides an automatic procedure to implement and execute tests.

\subsubsection{Functional ontology requirements extraction}

The functional ontology requirements extraction refers to the activity of collecting the functional ontology requirements that the standard should fulfil. These requirements should be related to the domain that the standard should model. In order to analyse conformance, the requirements should define the concepts and relations that must be defined in the domain described by the standard.

This activity produces a set of functional ontology requirements that could be materialised as competency questions or natural language statements. Moreover, in order to ease the requirements proposal, the CORAL Corpus, which is described in Section 4.1 can also be used as a guide on how to write requirements.

Ontology requirements should be grouped into one or more topics that summarise the topic related to them, e.g., the requirement "What is a gateway?" can be associated with the topic "Gateway". This grouping process facilitates the analysis of conformance once the tests results are obtained. To that end, each requirement should be tagged 
according to the topic it belongs to.

In a conformance testing scenario, the higher the number of requirements and the more concise and unambiguous the requirements are, the more accurate the identification of overlaps and conflicts between the standard and the ontology to be analysed. Moreover, for conformance testing to be useful, the requirements need to be unambiguous. Ontology engineers must guarantee that the defined requirements are correct and complete since they will be used in order to check conformance with the associated ontology. However, the CORAL corpus (detailed in Section 4.1) shows that multiple requirements that are published online are ambiguous and can lead to several implementations in an ontology. In that situation, the results obtained by the conformance analysis could be incorrect.

\subsubsection{Test design}

As defined in the ontology verification testing method (Chapter (4), during the test design step the desired behaviour of each requirement, i.e., their goal in the ontology, is extracted and formalised into the set of supported test expressions presented in Table 4.5. Following the ISO/IEC 9646 vocabulary, these test expressions represent the TPs, which indicate what is going to be tested in the ontology.

These TPs are defined without any information (e.g., URIs or labels) related to the ontology in which such test will be executed. Therefore, they can be considered abstract, being independent of the standard from which they are extracted. As an example, the requirement that states "An IoT gateway is a digital entity", which is extracted from the ISO/IEC 30141:2017 (ISO, 2017), is associated with the TP "Gateway subClassOf DigitalEntity". Since this TP does not include any URI related to the terms in the tests it can be executed on any ontology.

Each test design includes the identifier, the TP, the requirement and associated topic. The set of test designs that are generated during this activity constitutes the Abstract Test Suite (ATS).

The ATS must be stored in an RDF file by using the Verification Test Case ontology (Chapter 4.3), which describes test suites and test designs, including all the information related to them, such as the requirement associated, the TPs, the tag according to the topic to which the requirement belongs and the standard associated with the test. This ATS should be published online so that ontology engineers can use it to check whether their ontologies passed such tests and, consequently, are conformant to the standard. Moreover, published ATSs can also be understood as a formal documentation of the standard. 


\subsubsection{Test implementation}

In order to implement each test in the ATS, the implementation method described in Section 4.2 .2 is followed. These implementations generated from the method contain (1) a precondition, which is a SPARQL query that check that the terms in the test are included in the ontology; (2) a test preparation, which are a set of assertion added to the ontology in order to prepare all the temporal terms needed for executing the tests; and (3) the set of assertions to be added to the ontology in order to check the behaviour of the ontology.

The separation between the test design and the test implementation allows increasing the maintainability of tests. It is possible to change the implementation of tests without changing the test designs and, therefore, without changing the ATSs associated with the standards.

Table 6.1 summarises an example of implementation for the ontology requirement "An Io T gateway is a digital entity" presented in the previous section, which is associated with the TP "Gateway subClassOf DigitalEntity". The assertions in Table 6.1, which represent the axioms to be added to the ontology, are written using Description Logics syntax (Baader et al. 2008). The set of test implementations associated with the ATS constitutes the Executable Test Suite (ETS). The tests in the ETS are still abstract, since they do not include any information about the ontology on which the tests are going to be executed.

Table 6.1: Implementation of a test expression for checking subsumption between the classes Gateway and DigitalEntity

\begin{tabular}{|c|c|c|c|}
\hline Requirement: & \multicolumn{3}{|l|}{ An IoT is a digital entity } \\
\hline Test purpose: & \multicolumn{3}{|l|}{ Gateway subClassOf DigitalEntity } \\
\hline Type: & 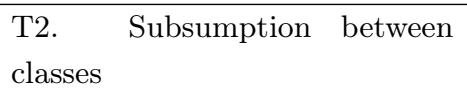 & Related to: & Classes \\
\hline \multicolumn{2}{|c|}{ Test precondition } & \multicolumn{2}{|r|}{ Test preparation } \\
\hline \multicolumn{2}{|c|}{ ASK $\{$ Class(Gateway), Class(DigitalEntity) $\}$} & \multicolumn{2}{|c|}{$\begin{array}{l}\text { (S 1.1) Declaration of } \neg \text { Gateway } \\
\text { (S 1.2) Declaration of } \neg \text { DigitalEntity }\end{array}$} \\
\hline \multicolumn{4}{|c|}{ Assertions to test the ontology behaviour } \\
\hline \multicolumn{2}{|r|}{ Axiom } & \multicolumn{2}{|c|}{ Expected status after adding the axiom } \\
\hline \multicolumn{2}{|c|}{ (S 2) Gateway’ $\sqsubseteq \neg$ Gateway $\sqcap$ DigitalEntity } & \multicolumn{2}{|c|}{ Consistent ontology } \\
\hline \multicolumn{2}{|c|}{ (S 3) Gateway’ $\sqsubseteq$ Gateway $\sqcap \neg$ DigitalEntity } & \multicolumn{2}{|c|}{ Unsatisfiable class } \\
\hline \multicolumn{2}{|c|}{ (S 4) Gateway’ $\sqsubseteq$ Gateway $\sqcap$ DigitalEntity } & \multicolumn{2}{|c|}{ Consistent ontology } \\
\hline
\end{tabular}




\subsubsection{Test execution}

Once the ETS is generated, each test implementation can be executed on an ontology. Following the test execution activity described in Section 4.2.3, this activity consists of three steps: (1) the execution of the query that represents the preconditions, (2) the addition of the axioms which declare the auxiliary terms, and (3) the addition of the assertions. After the addition of each axiom, a reasoner is executed to report the status of the ontology, i.e., whether the ontology is consistent, inconsistent or has unsatisfiable classes. The addition of the auxiliary axioms needs to always lead to a consistent ontology. However, in the case of the assertions, the agreement between the reasoner status after the addition of all the axioms and the status indicated in the test implementation determines whether the ontology satisfies the TP.

During this activity, each test implementation in the ETS should be first completed with the information related to the ontology in order to be executed. To that end, a glossary of terms must be generated manually or automatically in order to map each term in the test with a term in the ontology. Therefore, the terms that are defined in the ontology in which the test implementation will be executed, e.g., Gateway, are collected and associated with a URI in the ontology, e.g., <http://example.org/ontology\#Gateway>. Then, using these associations, the terms in the test implementation are translated into a term in the ontology. Consequently, the ETS can be executed on the ontology although it was extracted from a standard specification document.

Also following the test execution activity described in Section 4.2.3, the conformance testing method provides four possible results for each test implementation in the ETS and each ontology, namely, passed, undefined terms, absent and conflict results.

As an example, the test implementation described in Table 6.1. which is associated with a requirement extracted from the ISO/IEC 30141, can be executed on an ontology with URI < http://example.org/ontology\#>. If the ontology defines the terms Gateway and Digital Entity, as well as a subsumption relation between them, then the result of this execution should lead to the passed result and, therefore, the test design is passed and the requirement associated with the ISO/IEC 30141 standard would be satisfied by the ontology.

\subsubsection{Test results analysis}

In order to extract more information from the tests and to determine up to what extent an ontology conforms to a standard, a new activity is defined in this method, i.e., test results analysis. During this activity, the results of the test execution are analysed with 
the aim of identifying the degree of conformance of the ontology regarding the standard.

In order to analyse such conformance, the following information extracted from different artefacts involved in the verification process, i.e., the requirements, the tests and the ontology, can be obtained. The metrics used to extract such information are defined in Chapter 5 .

- How many tests included in the ATS associated with the standard are satisfied by the ontology. The Passed Test Percentage (PassedTestPtc) metric should be calculated to obtain this information. The higher the percentage of this metric, the greater the coverage of the ontology regarding the standard specification document.

- How many terms defined in the ATS associated with the standard are out of scope of the ontology. The Undefined Test Percentage (UTestPtc) metric should be calculated to obtain this information. The higher the percentage of this metric, the greater the number of standard terms that are out of scope of the ontology.

- How many relations in the ATS associated with the standard are not included in the ontology. The Absent Test Percentage (AbsentTestPtc) metric should be calculated to obtain this information. The higher the percentage of this metric, the greater the absent relations and restrictions in the ontology.

- How many tests in the ATS associated with the standard are not passed by the ontology. The Failed Test Percentage (FailedTestPtc) metric should be calculated to obtain this information. The higher the percentage of this metric, the greater the conflicts between the standard and the ontology.

- How many requirements in a standard specification document are satisfied by the ontology, since sometimes a requirement needs more than one test to be checked. The Covered Requirements Percentage (CovReqPtc) metric should be calculated to obtain this information. The higher the Covered Requirements Percentage metric, the greater the coverage between the standard and the ontology.

- How many requirements associated with a standard specification have a conflict with the ontology. The Requirements Fault Percentage (ReqFaultPtc) metric should be calculated to obtain this information, which in this case refer to those requirements of the standard that have a conflict with the ontology. Similarly to the Test Failed Percentage metric, the higher the percentage of this metric, the greater the number of conflicts between the standard and the ontology. 
- How many terms included in the standard are also defined in the ATS. In the conformance testing scenario, having this information helps to analyse the completeness of the requirements specification used in the testing process. The Test Effectiveness Ratio (TestEffRatio) metric should be calculated to obtain this information. The higher the percentage of this metric, the more complete the requirements specification will be. However, it should be considered that due to modelling decisions or ontology design patterns (Gangemi and Presutti, 2009) new terms can be added to the ontology but avoided in the requirements.

Using the previous metrics, the conformance degree can be determined through the following steps. Each step can be related to the requirements topics (topic level) or to the entire set of requirements (general level):

1. To identify the requirements of the standard whose tests are passed by the ontology, i.e., the coverage between the standard and the ontology. The Passed Test Percentage and Covered Requirements Percentage metrics facilitate the identification of such coverage. The Test Effectiveness Ratio metric provides an overview of the completeness of the requirements specification, which can also be included in the coverage analysis.

2. To identify the requirements whose tests result in undefined terms, i.e., the information that is included in the standard but is out of the scope of the ontology to be analysed.

3. To identify the requirements whose tests result in absence, i.e., the information related to restrictions and relations that are defined in the standard but not in the ontology. The Absent Test Percentage metric provides an overview of the percentage of tests with absent relations.

4. To identify the requirements whose tests result in conflict, i.e., the incompatibilities between the standard and the ontology. The Failed Test Percentage and Requirement Fault Percentage metrics identify the percentage of incompatible tests and requirements between the standard and the ontology to be analysed.

As an example of the degree of conformance that can be obtained with this information, if a set of requirements for a standard is divided into three topics, e.g., (1) Devices, (2) Users and (3) Services, it could be determined that the ontology to be analysed has a Covered Requirements Percentage of $60 \%$ for the first topic, but a $0 \%$ for the second 
and third topic. In this case and with this information it can be concluded that there is coverage between the standard and the ontology only for the specification of devices.

Based on these results, the conformance report is generated. The goal of this conformance report is to inform about the degree of conformance of a given ontology by using all the information obtained during the conformance analysis. Inspired by the ISO/IEC 9646, such report includes the following fields:

- The ontology to be analysed.

- The standard for which the analysis has been performed.

- The ATS together with its results.

- The ETS generated from the ATS.

- The glossary of terms used during the execution of the ETS.

- The obtained metrics for each topic or for the complete list of requirements.

- A clause that states the conformance status, which could include the overall conformance and the conformance divided by topic.

- A clause that provides information about those requirements related to the standard that are in conflict with the ontology.

The information provided by the conformance report can be used for requesting changes or for identifying conflicts between the developed ontology and the standard. Moreover, it can also be used to identify mappings and potential terms for reuse, as well as for guaranteeing interoperability between the standard or standard topics and the ontology.

\subsubsection{Technological support: Themis}

The tool Themis, previously described in Chapter 4, also supports the conformance testing method presented in this chapter. The requirements extraction, the test design and the analysis of the results are out of scope of Themis and should be done manually.

Themis allows the execution of an abstract test on a particular ontology. To that end, Themis needs a glossary of terms for executing such tests to map each term in the test with the corresponding term in the ontology to be analysed. Themis creates automatically this glossary of terms, which should be revised by the person in charge of the testing process, in order to modify it if needed. If there is a term that is in the test 
but not in the ontology, there will not exist a mapping for the term in the glossary, and it will be detected as an undefined term in the ontology. In this conformance scenario, the glossary of terms is crucial in order to correctly execute the abstract tests on a particular ontology.

\subsection{Method for minimum common knowledge identifica- tion}

When analysing a particular domain, it can be observed that several standards coexist. As an example, in the Internet of Things (IoT) domain the ETSI developed the SAREF ontology for smart applications (ETSI, 2020), the W3C developed the SSN ontology for describing sensors, their observations, and related procedures (Haller et al., 2019), and the oneM2M organisation developed the oneM2M base ontology for providing syntactic and semantic interoperability of oneM2M platforms with external systems oneM2M 2016). All these mentioned ontologies are related to the IoT domain; however, they are oriented to the description of different aspects in such domain.

Since reusing other ontologies is a key activity during ontology development, ontology engineers should be aware of how a set of standards covers their domain, identifying their scope, their overlaps, their differences and their conflicts. This information is valuable for identifying potential mappings and needs that are not supported by existing standards or that are defined in more than one. To that end, this section introduces a method for common knowledge identification between a set of standards, i.e., for the identification of the overlaps that are shared among their specifications. These standards can be ontologies or non-ontological specification documents that define data models.

This minimum common knowledge identification method is grounded on the conformance testing method described in the previous section. The activities related to the test design, implementation and execution are reused from the testing method for ontology verification (Chapter 4). However, the test execution activity is adapted to the particular scenario of minimum common knowledge identification in order to support the execution of all the ATSs on all the standards. The generation of ontologies for data models without an ontology associated, the generation of shared glossaries of terms and the analysis of the results are novel activities defined in this method. A more detailed description related to the differences between these two methods is described in the following sub-sections. 


\subsubsection{Activities within the minimum common knowledge identifica- tion method}

To carry out this minimum common knowledge identification method, the functional ontology requirements associated with all the standards to be analysed should be extracted to generate an ATS from each of them. Then, these ATSs are executed on all the standards, and the obtained results are analysed to determine the overlaps and differences between them.

The overview of the method for identifying the minimum common knowledge between standards is summarised in Figure 6.2. This method is grounded on the conformance testing method presented in Section 6.1 and, consequently, the activities are similar. However, in this scenario all the ATSs are executed on all the standards.

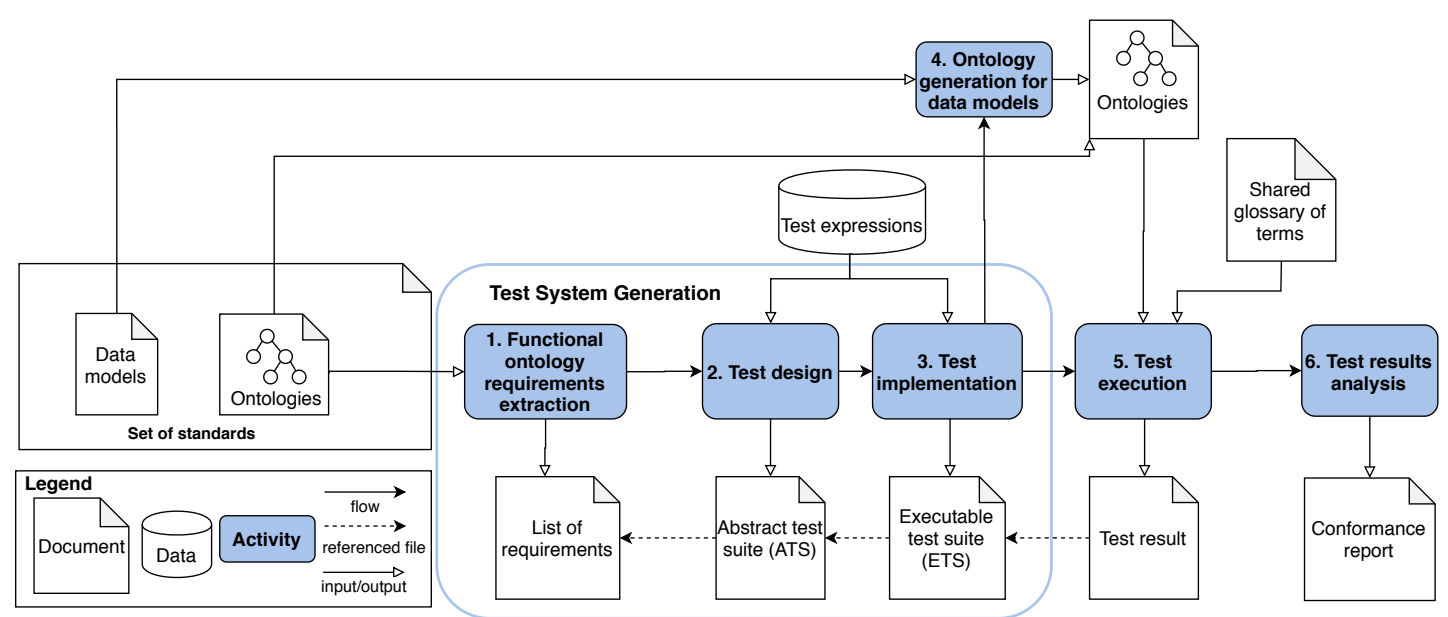

Figure 6.2: Conformance approach for identifying the minimum commitment between ontologies

\subsubsection{Generation of ontologies from data models}

The main challenge of this method is to execute the ATSs in every standard, even in those without any associated ontology, e.g., data models described in ISO/IEC norms. Therefore, an ontology is built from the ATS related to the standards that are not specified through an ontology to solve this issue.

To be able to automatically build an ontology from an ATS, the goals of the supported TPs that can be included in an ATS (Table 4.5) and that indicate the knowledge that should be defined in the standard, e.g., a subsumption relation between two classes, were collected and analysed. Based on this expected knowledge, a set of OWL axioms 
was associated with each supported TP. Table 6.2 shows an example of an association between TPs and axioms for three different TPs. The complete list of associations between the tests and axioms to generate the ontology is shown in Annex B.

\begin{tabular}{|l|l|l|}
\hline Goal & Test purpose & Axioms \\
\hline T1. Class definition & {$[$ ClassA] type Class } & :A rdf:type owl:Class . \\
\hline $\begin{array}{l}\text { T2. Subsumption relation between } \\
\text { classes A and B }\end{array}$ & [ClassA] subclassOf [ClassB] & $\begin{array}{r}\text { :A rdf:type owl:Class } \\
\text { :B rdf:type owl:Class; } \\
\text { rdfs:subClassOf :A }\end{array}$ \\
\hline $\begin{array}{l}\text { T3. Disjointness between two } \\
\text { classes }\end{array}$ & [ClassA] disjointWith [ClassB] & $\begin{array}{c}\text { :A rdf:type owl:Class } \\
\text { :Bdf:type owl:Class; } \\
\text { owl:disjointWith :A . }\end{array}$ \\
\hline
\end{tabular}

Table 6.2: Example of association between tests and axioms

These associations between a TP and a set of OWL axioms allows to automatically generate an ontology from an ATS for those standards that do not have an ontology related. The collection of all the OWL axioms retrieved by all the TPs in an ATS constitutes the ontology to be used in the method.

\subsubsection{Generation of a shared glossary of terms}

To automatically execute all ATSs on all the standards it is required to have a shared glossary of terms that maps the terms in each TP included in the ATSs and the terms in each standard. This shared glossary has to be used during the execution activity to complete the abstract tests with the corresponding URIs for each standard. An example of a shared glossary of terms is depicted in Table 6.3.

\begin{tabular}{|l|l|l|}
\hline Term in test & \multicolumn{1}{|c|}{ SAREF ontology } & \multicolumn{1}{c|}{ SSN ontology } \\
\hline Actuator & https://saref.etsi.org/core/Actuator & http://www.w3.org/ns/sosa/Actuator \\
\hline Sensor & https://saref.etsi.org/core/Sensor & http://www.w3.org/ns/sosa/Sensor \\
\hline
\end{tabular}

Table 6.3: Example of a shared glossary of terms

This glossary of terms should be generated either automatically or manually by the person in charge of the conformance testing process, who has to ensure that the glossary of terms is correct. It should be noted that the glossary of terms is a crucial element in the minimum common knowledge identification method since it allows to map each term in the ATSs with the terms in the standard. 


\subsubsection{Analysis of the results}

The output of the execution activity consists of the results obtained after the execution of each ATS in each standard. This hinders the test result analysis since more results have to be analysed. However, the same steps that were detailed in Section 6.1.1.5 can be followed for analysing these results. The adoption of this method allows to identify different layers of knowledge between standards, namely (Cuenca et al., 2019):

- The common knowledge, which represents the knowledge shared by the set of analysed models. In this case, the common knowledge represents the requirements that are satisfied by all the ontologies to be analysed.

- The variant-domain knowledge, which represents the knowledge that is common to more than one model, even though it is not shared by all of them. In this case, the variant-domain knowledge represents the requirements that are satisfied by more than two ontologies of the set of ontologies to be analysed.

- The domain-task knowledge, which represents the lower and most specific knowledge. In this case, the domain-task knowledge represents the requirements that are satisfied by only one ontology of the set of ontologies to be analysed.

These layers determine the knowledge that is common and the knowledge that is particular for each standard, identifying its scope and the commonalities between a set of standards. In addition to these layers, during the analysis of results the requirements that create conflicts between standards can also be identified.

\subsubsection{Technological support: Themis}

An extension of the tool Themis ${ }^{40}$ has been developed that, before executing the tests, automatically generates ontologies from the associated ATS for those standards without a related ontology. The requirements extraction, the test design and the analysis of the results are out of scope of this extension of Themis and should be done manually.

This extension of Themis uses the information presented in Annex B in order to generate the required ontologies based on the tests. Although these generated ontologies are not complete, they are enough for this scenario since they include the information that is described in the requirements. Once these ontologies are generated, Themis executes all the tests in the ATSs on all the ontologies introduced as an input following the conformance testing method.

\footnotetext{
${ }^{40}$ The code and distribution of the extension are available in the GitHub repository: https:// github.com/albaizq/ThemisForConformance
} 



\section{Chapter 7}

\section{Evaluation}

The main objective of this thesis is to contribute to the state of art in the ontology verification field by providing a new testing method for ontology engineers and practitioners that could also be used for extracting metrics and for analysing conformance. This chapter presents the evaluation of the main contributions of this thesis (Section 3.2 through their hypotheses (Section 3.3). In particular, this chapter presents:

- The evaluation of the method for ontology verification testing (Section 7.1).

- The evaluation of the set of metrics derived from the testing process artefacts (Section 7.2).

- The evaluation of the method for ontology conformance testing (Section 7.3).

\subsection{Evaluation of the method for ontology verification test- ing}

Chapter 4 presented the testing method for ontology verification that uses a testing language based on lexico-syntactic patterns. This section describes the empirical analysis performed in order to validate whether the use of testing languages helps ontology engineers and practitioners during the ontology verification process. The following hypothesis is made for this evaluation (Section 3.3):

- H1. Using a testing language for defining tests based on functional requirements facilitates the ontology testing process regarding the reduction of errors in users that are familiar and expert in OWL. 
With the aim of determining whether the use of a testing language for defining tests facilitates the ontology testing process, Themis, the technological support for the testing process presented in Section 4.4, was compared through a user evaluation to other tools in the state of the art for ontology verification. Some of these tools also use a testing language for the definition of tests. Different participants with different ontology development expertise participated in such evaluation, providing feedback regarding the verification tools and their usability.

\subsubsection{Experimental design}

The goal of this evaluation was to compare ontology verification tools that use testing languages with tools that do not. To that end, each participant in this experiment had to verify whether a given ontology satisfied a set of ontology requirements with one of these tools. Depending on the tool, participants had to execute tests or to browse the terms and axioms in the ontology to check whether the requirements were satisfied.

For the purpose of this evaluation, a set of 30 ontology requirements written in natural language was defined and, based on them, an ontology was developed and published 41 These requirements included diverse restrictions and had different complexities, such as simple requirements that ask for a hierarchy and complex requirements associated to cardinalities or to several relations between terms. Moreover, only some of the requirements were satisfied by the ontology. Participants had to identify which of them were satisfied by the ontology and which were not. The list of requirements used in this experiment is shown in Table 7.1 .

Table 7.1: List of requirements

\begin{tabular}{|l|l|l|l|}
\hline Identifier & Type & Requirement & Satisfied \\
\hline R.1 & Hierarchy & A smoke sensor is a type of sensor & Yes \\
\hline R.2 & Disjointness & Open commands differ from close commands & No \\
\hline R.3 & Terms relation & A switch consists of only an actuator & No \\
\hline R.4 & Hierarchy & A meter is a type of device and physical thing & No \\
\hline R.5 & Disjointness & A sensor cannot be an actuator & No \\
\hline R.6 & Terms relation & $\begin{array}{l}\text { An agent can only interact using an applica- } \\
\text { tion }\end{array}$ & No \\
\hline R.7 & Cardinality & A device has one or more functions & Yes \\
\hline R.8 & Instance & Drying is an example of task & Yes \\
\hline R.9 & Cardinality & Each task is accomplished by at least 2 devices & No \\
\hline R.10 & Cardinality & A service is offered by exactly 1 device & No \\
\hline R.11 & Terms relation and union & $\begin{array}{l}\text { A device can only have typical consumption of } \\
\text { energy or power }\end{array}$ & Yes \\
\hline
\end{tabular}

\footnotetext{
${ }^{41}$ https://w3id.org/def/themisEval\#
} 
Table 7.1 - Continued from previous page

\begin{tabular}{|c|c|c|c|}
\hline Identifier & Type & Requirement & Satisfied \\
\hline R.12 & Terms relation and hierarchy & $\begin{array}{l}\text { An actuator, which is a type of device, has an } \\
\text { actuating function }\end{array}$ & Yes \\
\hline R.13 & Terms relation & An illuminance unit observes some light & No \\
\hline R.14 & Hierarchy and disjointness & $\begin{array}{l}\text { Among the different types of commands there } \\
\text { are: open command and stop command; which } \\
\text { are also disjoint }\end{array}$ & No \\
\hline R.15 & Cardinality and terms relation & $\begin{array}{l}\text { A device has one or more functions and these } \\
\text { functions accomplish some tasks }\end{array}$ & No \\
\hline R.16 & Cardinality and terms relation & $\begin{array}{l}\text { A sensor measures at least one property and } \\
\text { these properties relate to measurement }\end{array}$ & No \\
\hline R.17 & Hierarchy & $\begin{array}{l}\text { Devices are divided into two different types: } \\
\text { actuator and sensor }\end{array}$ & Yes \\
\hline R.18 & Terms relation & An agent participates in an organisation & Yes \\
\hline R.19 & Terms relation & An agent is participant in an event & No \\
\hline R.20 & Instance & $\begin{array}{l}\text { The accumulated energy produced is an exam- } \\
\text { ple of measurement }\end{array}$ & No \\
\hline R.21 & Hierarchy & A sensor is a device & Yes \\
\hline R.22 & Hierarchy & A sensor is a physical thing & No \\
\hline R.23 & Cardinality & A sensor has to accomplish at least 1 task & Yes \\
\hline R.24 & Cardinality & A sensor must make at least 1 measurement & No \\
\hline $\mathrm{R} .25$ & Terms relation & $\begin{array}{l}\text { A light switch can consist only in several de- } \\
\text { vices }\end{array}$ & No \\
\hline R.26 & Terms relation & A washing machine consists of some sensor & No \\
\hline R.27 & Disjointness & An agent cannot be a device & No \\
\hline R.28 & Disjointness & A temperature sensor cannot be an actuator & No \\
\hline R.29 & Cardinality & An indicator relates to at least 2 properties & No \\
\hline R.30 & Cardinality & Power is controlled by exactly 1 device & No \\
\hline
\end{tabular}

Based on the results of the participants, the verification tools can be compared in two aspects, namely, the errors made by the participants and the time of the verification process, since it can influence such errors.

In order to check the errors made by the participants, the provided results, i.e., the set of satisfied or unsatisfied requirements, were analysed in order to identify for each tool:

- Incorrect answers, i.e., to affirm that a requirement is satisfied by an ontology when it is not or vice versa.

- Correct answers, i.e., to affirm that a requirement is not satisfied by an ontology and it is not or that a requirement is satisfied by an ontology and it is.

- Unsolvable requirements, which are requirements that could not be verified by participants. In this analysis, it was asked to the participants to leave a requirement as "unsolvable" if they had to spend more than 5 minutes to check whether it is 
satisfied or not. In addition, unsolvable requirements refer to those requirements that were not understood by the participants.

In order to check the time spent by the participants for each tool, during the experiment it was calculated the time spent by each participant in verifying each requirement.

With the aim of comparing the tools, an online questionnaire to be fulfilled by the participants of the experiment was created. Such questionnaire collected data from participants and their results regarding the verification process.

First, the questionnaire asked participants to add their demographic data, including expertise in the OWL language and in software development. Moreover, participants were asked about previous experience with the tools analysed in the experiment.

Afterwards, the questionnaire asked the participants about the verification process for each requirement in the experiment. The following information had to be added by each participant for each requirement:

- The test or technique that was used to verify the requirement (e.g., the use of a reasoner or an editor browser).

- Based on the results of the test or the technique used, participants had to indicate for each requirement: (1) if the requirement is satisfied; (2) if there are terms in the requirement that are not included in the ontology; (3) if there is any absent restriction; (4) if there is a conflict between the requirement and the analysed ontology; or (5) if the participant does not know how to verify the requirement.

- Any feedback or comment that the participants wanted to report related to the tool, the requirement or the tests associated with it.

Besides, the questionnaire collects automatically the time spent by each participant in indicating whether each requirement is satisfied by the ontology or not.

Once the participants finished fulfilling the questionnaire, a USE questionnaire (Lund, 2001) was sent to them with questions related to the usefulness, satisfaction and usability of the tools. The questionnaire included 11 qualitative questions divided into the three categories, namely, usefulness, satisfaction and usability. Within the questionnaire, which is included in Annex C, questions are rated on a Likert scale from 1 (strongly disagree) to 5 (strongly agree). The questionnaire also included questions for listing the most positive and negative aspects of the tools, and allowed participants to send additional comments regarding the usability of the tools. 


\subsubsection{Results and discussion}

To perform this experiment, the tools Themis (described in Section 4.4), TDDOnto2 (described in Section 2.3.6) and Protég ${ }^{42}$ were used by the participants. Both Themis and TDDOnto2 are tools that use testing languages to define tests to verify an ontology. Themis uses the testing language presented in Section 4.2, which is based on the OWL Manchester Syntax, and provides guides to help users in the definition of tests. Regarding TDDOnto2, its tests are based on axioms written in the OWL Manchester syntax. TDDOnto2 relies on users knowledge and, therefore, no guides are provided to help users, although it includes an autocompletion service. Protégé is an ontology editor that allows to visualise and browse the hierarchy of classes and their properties in an ontology.

In this evaluation, 30 participants were involved (10 participants per tool), among which there were experts and non-experts in the OWL language (participants that were familiar and not-familiar with OWL). Tables 7.2 and 7.3 summarise the expertise of the participants in ontology and software development, as well as their expertise in the tools.

Table 7.2: OWL expertise of participants in the experiment

\begin{tabular}{|l|l|l|l|l|l|l|}
\hline \multirow{2}{*}{ Tool } & \multicolumn{3}{c|}{ OWL expertise } & \multicolumn{3}{c|}{ OWL Manchester Syntax expertise } \\
\cline { 2 - 7 } & Not familiar & Familiar & Expert & Not familiar & Familiar & Expert \\
\hline Themis & 1 & 7 & 2 & 4 & 6 & 0 \\
\hline TDDOnto2 & 1 & 6 & 3 & 8 & 1 & 1 \\
\hline Protégé & 6 & 3 & 1 & 8 & 1 & 1 \\
\hline
\end{tabular}

Table 7.3: Development expertise of participants in the experiment

\begin{tabular}{|l|l|l|l|l|l|l|}
\hline \multirow{2}{*}{ Tool } & \multicolumn{2}{|c|}{ Software development expertise } & \multicolumn{2}{c|}{ Tool expertise } \\
\cline { 2 - 7 } & Not familiar & Familiar & Expert & Not familiar & Familiar & Expert \\
\hline Themis & 1 & 7 & 2 & 9 & 1 & 0 \\
\hline TDDOnto2 & 4 & 3 & 3 & 8 & 2 & 0 \\
\hline Protégé & 4 & 4 & 2 & 5 & 3 & 2 \\
\hline
\end{tabular}

As shown in these tables, the majority of the participants are familiar with the OWL language, but they do not have background on the OWL Manchester Syntax. Moreover, there are several participants that are also familiar with software development, which can help to understand the testing process. Finally, the majority of the participants had not used any of the testing tools until the experiment.

$4^{42}$ https://protégé.stanford.edu/ 


\subsubsection{Time analysis}

The experiment was planned to last around one hour and a half. It should be noted that not every participant answered the 30 requirements because of time issues, since they spent more than the time planned.

Figures 7.1 to 7.3 show the obtained results with regards to the time spent per requirement in the experiment, indicating the average seconds spent per requirement in each of the tools.

It should be noted that in Figure 7.1 Themis does not have values from requirements R.14 to R.30. This is because the only participant not familiar with the OWL language that used Themis could not finish the questionnaire because of time issues.



Figure 7.1: Comparison of the time spent per requirement for participants not familiar with the OWL language

From these figures it can be observed that, regardless of the expertise of the participants, Protégé has a stable time spent per requirement during the verification process, while both Themis and TDDOnto2 have a learning curve. As an example, in Figure 7.3 it can be observed that R.1 for TDDOnto2 takes more than twice the time spent in Protégé for participants experts in the OWL language. However, after R.19 the time spent in the three tools is similar, although the complexity of these requirements is not different from the previous ones. Furthermore, it can be observed that even if the time is higher during the first requirements, it decreases over time for all participants. 


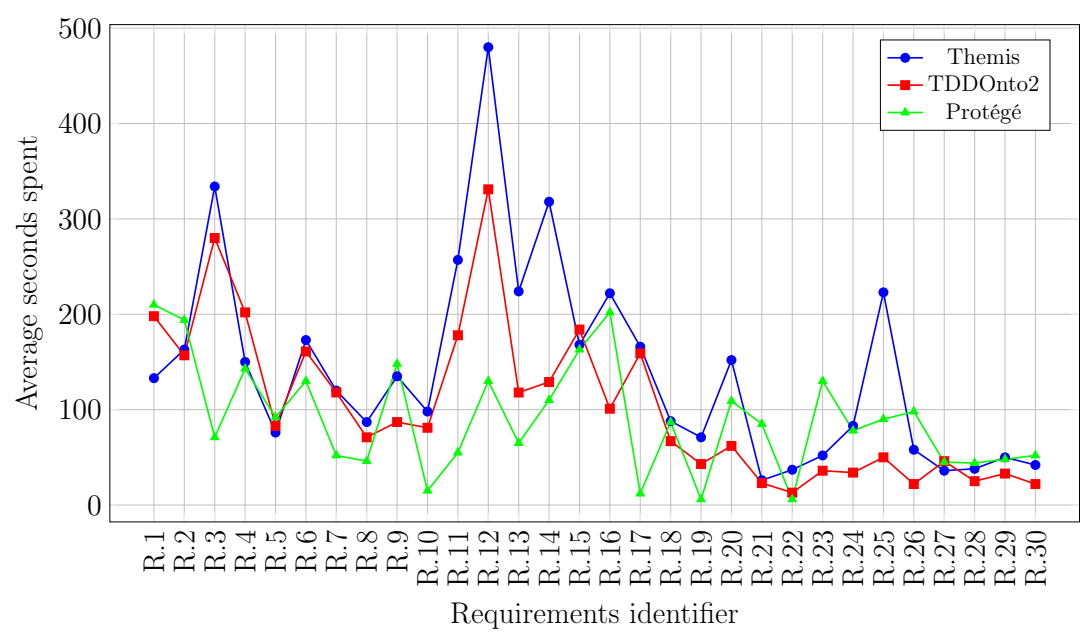

Figure 7.2: Comparison of the time spent per requirement for participants familiar with the OWL language

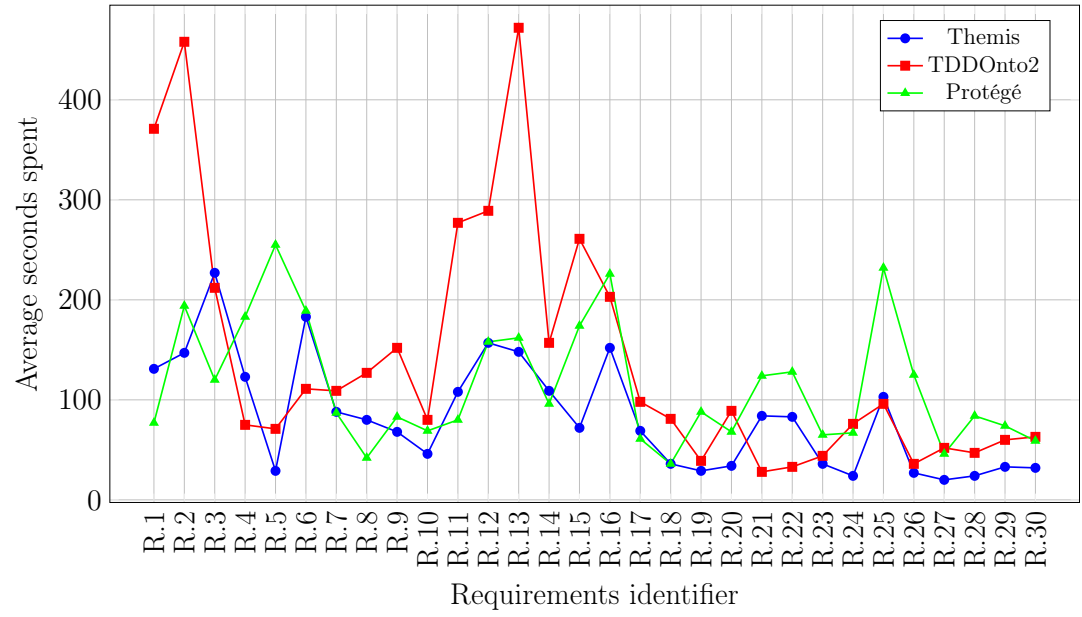

Figure 7.3: Comparison of the time spent per requirement for participants experts with the OWL language

\subsubsection{Correctness analysis}

During this empirical analysis the answers (incorrect and correct) given by the users were also collected. Tables 7.4 to $\left.7.4\right|^{3}$ detail the results collected from the analysis, grouped by the type of participant and by tool. These figures include the percentage of correct and incorrect answers for each requirement and the number of unsolved requirements,

\footnotetext{
${ }^{43}$ For the sake of readability, the numbers of all the tables in this section are rounded. Besides, $\mathrm{C}$ refers to correct results, I to incorrect results and $U$ to unresolvable results.
} 
i.e., those requirements that the participants did not know how to verify.

Table 7.4: Percentage of correctness results for Themis

\begin{tabular}{|c|c|c|c|c|c|c|c|c|c|}
\hline \multirow{3}{*}{ Requirement } & \multicolumn{9}{|c|}{ Participants } \\
\hline & \multicolumn{3}{|c|}{ Not familiar } & \multicolumn{3}{|c|}{ Familiar } & \multicolumn{3}{|c|}{ Expert } \\
\hline & $\mathrm{C}$ & I & $\mathbf{U}$ & $\mathbf{C}$ & I & $\mathbf{U}$ & $\mathrm{C}$ & I & $\mathbf{U}$ \\
\hline R.1 & $0 \%$ & $100 \%$ & $0 \%$ & $50 \%$ & $50 \%$ & $0 \%$ & $50 \%$ & $50 \%$ & $0 \%$ \\
\hline R.2 & $0 \%$ & $100 \%$ & $0 \%$ & $100 \%$ & $0 \%$ & $0 \%$ & $100 \%$ & $0 \%$ & $0 \%$ \\
\hline R.3 & $0 \%$ & $100 \%$ & $0 \%$ & $100 \%$ & $0 \%$ & $0 \%$ & $100 \%$ & $0 \%$ & $0 \%$ \\
\hline R.4 & $0 \%$ & $100 \%$ & $0 \%$ & $100 \%$ & $0 \%$ & $0 \%$ & $100 \%$ & $0 \%$ & $0 \%$ \\
\hline R.5 & $100 \%$ & $0 \%$ & $0 \%$ & $100 \%$ & $0 \%$ & $0 \%$ & $100 \%$ & $0 \%$ & $0 \%$ \\
\hline R.6 & $0 \%$ & $100 \%$ & $0 \%$ & $71 \%$ & $29 \%$ & $0 \%$ & $100 \%$ & $0 \%$ & $0 \%$ \\
\hline R.7 & $100 \%$ & $0 \%$ & $0 \%$ & $71 \%$ & $29 \%$ & $0 \%$ & $100 \%$ & $0 \%$ & $0 \%$ \\
\hline R. 8 & $100 \%$ & $0 \%$ & $100 \%$ & $43 \%$ & $58 \%$ & $0 \%$ & $100 \%$ & $0 \%$ & $0 \%$ \\
\hline R.9 & $0 \%$ & $100 \%$ & $0 \%$ & $71 \%$ & $29 \%$ & $0 \%$ & $50 \%$ & $50 \%$ & $0 \%$ \\
\hline R.10 & $100 \%$ & $0 \%$ & $0 \%$ & $86 \%$ & $14 \%$ & $0 \%$ & $100 \%$ & $0 \%$ & $0 \%$ \\
\hline R.11 & $0 \%$ & $100 \%$ & $0 \%$ & $100 \%$ & $0 \%$ & $0 \%$ & $100 \%$ & $0 \%$ & $0 \%$ \\
\hline R.12 & $100 \%$ & $0 \%$ & $0 \%$ & $42 \%$ & $42 \%$ & $14 \%$ & $50 \%$ & $50 \%$ & $0 \%$ \\
\hline R.13 & $100 \%$ & $0 \%$ & $0 \%$ & $57 \%$ & $29 \%$ & $14 \%$ & $50 \%$ & $50 \%$ & $0 \%$ \\
\hline R.14 & - & - & - & $86 \%$ & $14 \%$ & $0 \%$ & $50 \%$ & $50 \%$ & $0 \%$ \\
\hline R.15 & - & - & - & $100 \%$ & $0 \%$ & $0 \%$ & $50 \%$ & $50 \%$ & $0 \%$ \\
\hline R.16 & - & - & - & $71 \%$ & $0 \%$ & $29 \%$ & $100 \%$ & $0 \%$ & $0 \%$ \\
\hline R.17 & - & - & - & $0 \%$ & $100 \%$ & $0 \%$ & $50 \%$ & $50 \%$ & $0 \%$ \\
\hline R.18 & - & - & - & $100 \%$ & $0 \%$ & $0 \%$ & $100 \%$ & $0 \%$ & $0 \%$ \\
\hline R.19 & - & - & - & $100 \%$ & $0 \%$ & $0 \%$ & $100 \%$ & $0 \%$ & $0 \%$ \\
\hline R.20 & - & - & - & $71 \%$ & $29 \%$ & $0 \%$ & $50 \%$ & $50 \%$ & $0 \%$ \\
\hline R.21 & - & - & - & $86 \%$ & $14 \%$ & $0 \%$ & $0 \%$ & $100 \%$ & $0 \%$ \\
\hline R.22 & - & - & - & $100 \%$ & $0 \%$ & $0 \%$ & $100 \%$ & $0 \%$ & $0 \%$ \\
\hline R.23 & - & - & - & $86 \%$ & $14 \%$ & $0 \%$ & $100 \%$ & $0 \%$ & $0 \%$ \\
\hline R. 24 & - & - & - & $86 \%$ & $14 \%$ & $0 \%$ & $100 \%$ & $0 \%$ & $0 \%$ \\
\hline R.25 & - & - & - & $14 \%$ & $71 \%$ & $14 \%$ & $100 \%$ & $0 \%$ & $0 \%$ \\
\hline R.26 & - & - & - & $100 \%$ & $0 \%$ & $0 \%$ & $100 \%$ & $0 \%$ & $0 \%$ \\
\hline R.27 & - & - & - & $100 \%$ & $0 \%$ & $0 \%$ & $100 \%$ & $0 \%$ & $0 \%$ \\
\hline R.28 & - & - & - & $100 \%$ & $0 \%$ & $0 \%$ & $100 \%$ & $0 \%$ & $0 \%$ \\
\hline R.29 & - & - & - & $100 \%$ & $0 \%$ & $0 \%$ & $100 \%$ & $0 \%$ & $0 \%$ \\
\hline R.30 & - & - & - & $86 \%$ & $14 \%$ & $0 \%$ & $100 \%$ & $0 \%$ & $0 \%$ \\
\hline Average & $46 \%$ & $54 \%$ & $8 \%$ & $79 \%$ & $18 \%$ & $2 \%$ & $83 \%$ & $17 \%$ & $0 \%$ \\
\hline
\end{tabular}


Table 7.5: Percentage of correctness results for TDDOnto2

\begin{tabular}{|c|c|c|c|c|c|c|c|c|c|}
\hline \multirow{3}{*}{ Requirement } & \multicolumn{9}{|c|}{ Participants } \\
\hline & \multicolumn{3}{|c|}{ Not familiar } & \multicolumn{3}{|c|}{ Familiar } & \multicolumn{3}{|c|}{ Expert } \\
\hline & $\mathrm{C}$ & I & $\mathbf{U}$ & C & I & $\mathbf{U}$ & C & I & $\mathbf{U}$ \\
\hline R.1 & $50 \%$ & $50 \%$ & $0 \%$ & $100 \%$ & $0 \%$ & $0 \%$ & $100 \%$ & $0 \%$ & $0 \%$ \\
\hline R.2 & $100 \%$ & $0 \%$ & $0 \%$ & $83 \%$ & $17 \%$ & $0 \%$ & $67 \%$ & $33 \%$ & $0 \%$ \\
\hline R.3 & $100 \%$ & $0 \%$ & $0 \%$ & $67 \%$ & $33 \%$ & $0 \%$ & $100 \%$ & $0 \%$ & $0 \%$ \\
\hline R.4 & $100 \%$ & $0 \%$ & $0 \%$ & $100 \%$ & $0 \%$ & $0 \%$ & $100 \%$ & $0 \%$ & $0 \%$ \\
\hline R.5 & $0 \%$ & $50 \%$ & $0 \%$ & $0 \%$ & $100 \%$ & $0 \%$ & $33 \%$ & $67 \%$ & $0 \%$ \\
\hline R. 6 & $0 \%$ & $0 \%$ & $100 \%$ & $67 \%$ & $33 \%$ & $0 \%$ & $67 \%$ & $33 \%$ & $0 \%$ \\
\hline R.7 & $100 \%$ & $0 \%$ & $0 \%$ & 100 & $0 \%$ & $0 \%$ & $100 \%$ & $0 \%$ & $0 \%$ \\
\hline R. 8 & $100 \%$ & $0 \%$ & $100 \%$ & $67 \%$ & $33 \%$ & $0 \%$ & $100 \%$ & $0 \%$ & $0 \%$ \\
\hline R.9 & $100 \%$ & $0 \%$ & $0 \%$ & $50 \%$ & $50 \%$ & $0 \%$ & $67 \%$ & $33 \%$ & $0 \%$ \\
\hline R.10 & $100 \%$ & $0 \%$ & $0 \%$ & $83 \%$ & $17 \%$ & $0 \%$ & $100 \%$ & $0 \%$ & $0 \%$ \\
\hline R.11 & $0 \%$ & $100 \%$ & $0 \%$ & $17 \%$ & $83 \%$ & $0 \%$ & $67 \%$ & $33 \%$ & $0 \%$ \\
\hline $\begin{array}{l}\text { R.12 } \\
\end{array}$ & $0 \%$ & $0 \%$ & $100 \%$ & $67 \%$ & $33 \%$ & $0 \%$ & $100 \%$ & $0 \%$ & $0 \%$ \\
\hline R.13 & $100 \%$ & $0 \%$ & $0 \%$ & $67 \%$ & $17 \%$ & $17 \%$ & $33 \%$ & $67 \%$ & $0 \%$ \\
\hline R.14 & $100 \%$ & $0 \%$ & $0 \%$ & $83 \%$ & $17 \%$ & $0 \%$ & $67 \%$ & $33 \%$ & $0 \%$ \\
\hline R.15 & $0 \%$ & $100 \%$ & $0 \%$ & $333 \%$ & $33 \%$ & $33 \%$ & $33 \%$ & $67 \%$ & $0 \%$ \\
\hline R.16 & $0 \%$ & $0 \%$ & $100 \%$ & $83 \%$ & $0 \%$ & $17 \%$ & $100 \%$ & $0 \%$ & $0 \%$ \\
\hline R.17 & $0 \%$ & $0 \%$ & $100 \%$ & $33 \%$ & $50 \%$ & $17 \%$ & $67 \%$ & $33 \%$ & $0 \%$ \\
\hline R.18 & $100 \%$ & $0 \%$ & $0 \%$ & $50 \%$ & $50 \%$ & $0 \%$ & $67 \%$ & $33 \%$ & $0 \%$ \\
\hline R.19 & $100 \%$ & $0 \%$ & $0 \%$ & $100 \%$ & $0 \%$ & $0 \%$ & $100 \%$ & $0 \%$ & $0 \%$ \\
\hline R. 20 & $100 \%$ & $0 \%$ & $0 \%$ & $80 \%$ & $20 \%$ & $0 \%$ & $100 \%$ & $0 \%$ & $0 \%$ \\
\hline R.21 & $100 \%$ & $0 \%$ & $0 \%$ & $100 \%$ & $0 \%$ & $0 \%$ & $100 \%$ & $0 \%$ & $0 \%$ \\
\hline R. 22 & $100 \%$ & $0 \%$ & $0 \%$ & $100 \%$ & $0 \%$ & $0 \%$ & $100 \%$ & $0 \%$ & $0 \%$ \\
\hline R. 23 & $100 \%$ & $0 \%$ & $0 \%$ & $80 \%$ & $20 \%$ & $0 \%$ & $100 \%$ & $0 \%$ & $0 \%$ \\
\hline R.24 & $100 \%$ & $0 \%$ & $0 \%$ & $100 \%$ & $0 \%$ & $0 \%$ & $100 \%$ & $0 \%$ & $0 \%$ \\
\hline R.25 & $0 \%$ & $0 \%$ & $100 \%$ & $40 \%$ & $60 \%$ & $0 \%$ & $67 \%$ & $33 \%$ & $0 \%$ \\
\hline R. 26 & $0 \%$ & $0 \%$ & $100 \%$ & $100 \%$ & $0 \%$ & $0 \%$ & $100 \%$ & $0 \%$ & $0 \%$ \\
\hline R. 27 & $100 \%$ & $0 \%$ & $0 \%$ & $100 \%$ & $0 \%$ & $0 \%$ & $100 \%$ & $0 \%$ & $0 \%$ \\
\hline R. 28 & $100 \%$ & $0 \%$ & $0 \%$ & $80 \%$ & $20 \%$ & $0 \%$ & $100 \%$ & $0 \%$ & $0 \%$ \\
\hline R.29 & $100 \%$ & $0 \%$ & $0 \%$ & $80 \%$ & $20 \%$ & $0 \%$ & $100 \%$ & $0 \%$ & $0 \%$ \\
\hline R.30 & $100 \%$ & $0 \%$ & $0 \%$ & $80 \%$ & $20 \%$ & $0 \%$ & $100 \%$ & $0 \%$ & $0 \%$ \\
\hline Average & $68 \%$ & $10 \%$ & $23 \%$ & $72 \%$ & $24 \%$ & $6 \%$ & $81 \%$ & $16 \%$ & $0 \%$ \\
\hline
\end{tabular}


Table 7.6: Percentage of correctness results for Protégé

\begin{tabular}{|c|c|c|c|c|c|c|c|c|c|}
\hline \multirow{3}{*}{ Requirement } & \multicolumn{9}{|c|}{ Participants } \\
\hline & \multicolumn{3}{|c|}{ Not familiar } & \multicolumn{3}{|c|}{ Familiar } & \multicolumn{3}{|c|}{ Expert } \\
\hline & C & I & $\mathbf{U}$ & C & I & $\mathrm{U}$ & C & I & $\mathbf{U}$ \\
\hline R.1 & $100 \%$ & $0 \%$ & $0 \%$ & $100 \%$ & $0 \%$ & $0 \%$ & $100 \%$ & $0 \%$ & $0 \%$ \\
\hline R. 2 & $83 \%$ & $17 \%$ & $0 \%$ & $100 \%$ & $0 \%$ & $0 \%$ & $100 \%$ & $0 \%$ & $0 \%$ \\
\hline R.3 & $17 \%$ & $83 \%$ & $0 \%$ & $0 \%$ & $100 \%$ & $0 \%$ & $0 \%$ & $100 \%$ & $0 \%$ \\
\hline R.4 & $33 \%$ & $67 \%$ & $0 \%$ & $0 \%$ & $100 \%$ & $0 \%$ & $0 \%$ & $100 \%$ & $0 \%$ \\
\hline R.5 & $17 \%$ & $83 \%$ & $0 \%$ & $0 \%$ & $100 \%$ & $0 \%$ & $0 \%$ & $100 \%$ & $0 \%$ \\
\hline R.6 & $67 \%$ & $33 \%$ & $0 \%$ & $33 \%$ & $67 \%$ & $0 \%$ & $0 \%$ & $100 \%$ & $0 \%$ \\
\hline R.7 & $83 \%$ & $17 \%$ & $0 \%$ & $100 \%$ & $0 \%$ & $0 \%$ & $100 \%$ & $0 \%$ & $0 \%$ \\
\hline R. 8 & $83 \%$ & $17 \%$ & $0 \%$ & $33 \%$ & $67 \%$ & $0 \%$ & $100 \%$ & $0 \%$ & $0 \%$ \\
\hline R.9 & $50 \%$ & $50 \%$ & $0 \%$ & $100 \%$ & $0 \%$ & $0 \%$ & $100 \%$ & $0 \%$ & $0 \%$ \\
\hline R.10 & $17 \%$ & $83 \%$ & $0 \%$ & $0 \%$ & $100 \%$ & $0 \%$ & $100 \%$ & $0 \%$ & $0 \%$ \\
\hline R.11 & $83 \%$ & $17 \%$ & $0 \%$ & $100 \%$ & $0 \%$ & $0 \%$ & $100 \%$ & $0 \%$ & $0 \%$ \\
\hline R.12 & $67 \%$ & $33 \%$ & $0 \%$ & $33 \%$ & $67 \%$ & $0 \%$ & $0 \%$ & $100 \%$ & $0 \%$ \\
\hline R.13 & $83 \%$ & $17 \%$ & $0 \%$ & $67 \%$ & $33 \%$ & $0 \%$ & $0 \%$ & $100 \%$ & $0 \%$ \\
\hline R.14 & $67 \%$ & $33 \%$ & $0 \%$ & $67 \%$ & $33 \%$ & $0 \%$ & $100 \%$ & $0 \%$ & $0 \%$ \\
\hline R.15 & $67 \%$ & $33 \%$ & $0 \%$ & $0 \%$ & $100 \%$ & $0 \%$ & $0 \%$ & $100 \%$ & $0 \%$ \\
\hline R.16 & $83 \%$ & $17 \%$ & $0 \%$ & $67 \%$ & $33 \%$ & $0 \%$ & $100 \%$ & $0 \%$ & $0 \%$ \\
\hline R.17 & $0 \%$ & $100 \%$ & $0 \%$ & $0 \%$ & $100 \%$ & $0 \%$ & $0 \%$ & $100 \%$ & $0 \%$ \\
\hline R.18 & $100 \%$ & $0 \%$ & $0 \%$ & $100 \%$ & $0 \%$ & $0 \%$ & $0 \%$ & $100 \%$ & $0 \%$ \\
\hline R.19 & $83 \%$ & $67 \%$ & $0 \%$ & $33 \%$ & $67 \%$ & $0 \%$ & $0 \%$ & $100 \%$ & $0 \%$ \\
\hline R.20 & $50 \%$ & $33 \%$ & $17 \%$ & $67 \%$ & $33 \%$ & $0 \%$ & $0 \%$ & $100 \%$ & $0 \%$ \\
\hline R.21 & $100 \%$ & $0 \%$ & $0 \%$ & $67 \%$ & $33 \%$ & $0 \%$ & $0 \%$ & $100 \%$ & $0 \%$ \\
\hline R. 22 & $33 \%$ & $67 \%$ & $0 \%$ & $0 \%$ & $100 \%$ & $0 \%$ & $100 \%$ & $0 \%$ & $0 \%$ \\
\hline R.23 & $100 \%$ & $0 \%$ & $0 \%$ & $33 \%$ & $67 \%$ & $0 \%$ & $100 \%$ & $0 \%$ & $0 \%$ \\
\hline R.24 & $100 \%$ & $0 \%$ & $0 \%$ & $0 \%$ & $100 \%$ & $0 \%$ & $100 \%$ & $0 \%$ & $0 \%$ \\
\hline R. 25 & $33 \%$ & $33 \%$ & $33 \%$ & $0 \%$ & $100 \%$ & $0 \%$ & $100 \%$ & $0 \%$ & $0 \%$ \\
\hline R. 26 & $33 \%$ & $67 \%$ & $0 \%$ & $67 \%$ & $33 \%$ & $0 \%$ & $0 \%$ & $100 \%$ & $0 \%$ \\
\hline R. 27 & $33 \%$ & $67 \%$ & $0 \%$ & $0 \%$ & $100 \%$ & $0 \%$ & $0 \%$ & $100 \%$ & $0 \%$ \\
\hline R. 28 & $17 \%$ & $83 \%$ & $0 \%$ & $0 \%$ & $100 \%$ & $0 \%$ & $100 \%$ & $0 \%$ & $0 \%$ \\
\hline R. 29 & $67 \%$ & $33 \%$ & $0 \%$ & $100 \%$ & $0 \%$ & $0 \%$ & $0 \%$ & $100 \%$ & $0 \%$ \\
\hline R.30 & $50 \%$ & $50 \%$ & $0 \%$ & $33 \%$ & $67 \%$ & $0 \%$ & $100 \%$ & $0 \%$ & $0 \%$ \\
\hline Average & $60 \%$ & $40 \%$ & $2 \%$ & $43 \%$ & $57 \%$ & $0 \%$ & $50 \%$ & $50 \%$ & $0 \%$ \\
\hline
\end{tabular}


Checking the summarised results presented in Table 7.7, it can be concluded that the percentage of correct results is significantly higher in Themis and TDDOnto2 for users that are familiar or experts in OWL. For this range of users, i.e., familiar and experts, Themis and TDDOnto2 also present a significantly lower number of incorrect results.

For those users that were not familiar with OWL, there is not a high variability in the results of the tools (even if they are different). It should also be noted that Protégé had more participants that are not familiar with the OWL language than the other tools.

Table 7.7: Summary of the error results

\begin{tabular}{|c|c|c|c|c|c|c|c|c|c|}
\hline & \multicolumn{9}{|c|}{ Participants } \\
\hline & \multicolumn{3}{|c|}{ Not Familiar } & \multicolumn{3}{|c|}{ Familiar } & \multicolumn{3}{|c|}{ Expert } \\
\hline & $\mathrm{C}$ & I & $\mathbf{U}$ & $\mathrm{C}$ & I & $\mathrm{U}$ & $\mathrm{C}$ & I & $\mathrm{U}$ \\
\hline \multicolumn{10}{|c|}{ Themis } \\
\hline Average results & $46 \%$ & $54 \%$ & $8 \%$ & $79 \%$ & $18 \%$ & $2 \%$ & $83 \%$ & $17 \%$ & $0 \%$ \\
\hline \multicolumn{10}{|c|}{ TDDOnto2 } \\
\hline Average results & $68 \%$ & $10 \%$ & $23 \%$ & $72 \%$ & $24 \%$ & $6 \%$ & $81 \%$ & $16 \%$ & $0 \%$ \\
\hline \multicolumn{10}{|c|}{ Protégé } \\
\hline Average results & $60 \%$ & $40 \%$ & $2 \%$ & $43 \%$ & $57 \%$ & $0 \%$ & $50 \%$ & $50 \%$ & $0 \%$ \\
\hline
\end{tabular}

With these results, it can be concluded that, although Protégé is the tool with the most stable time spent per requirement, it is also the tool with the worst results with regards to the errors made during the verification process. In contrast, Themis and TDDOnto2 have a learning curve, but yield better results in terms of correctness for those users that are familiar and experts in OWL.

Apart from this, it should be noted that in the three tools there were some requirements that the participants could not test, although the number of these unsolved requirements is low. The requirements that had a higher percentage of unresolved results were R.12, R.13 and R.25 in the case of Themis, R.13, R.15, R.16 and R.17 in the case of TDDOnto2 and R.20 in the case of Protégé. Requirements R.12, R.13 and R.25 are associated with relations between terms in the ontology, R.15 and R.16 include also a cardinality restriction, R.17 is related to a hierarchy between classes, and R.20 is associated to the definition of an instance of a class. This motivates the analysis of the correctness results in terms of the type of requirement.

The majority of the requirements with a higher percentage of unresolved results are related to term relations since the participants could not identify which restrictions, e.g., the existential or universal ones, were associated to such requirements. 
Tables 7.8 to 7.10 show the results obtained from the three tools grouped by type of requirement. From these tables it can be observed that, in general, those requirements that include several restrictions, e.g., a hierarchy and disjoint classes, are the most difficult requirements to verify, since they have a higher percentage of incorrect results.

Regarding Themis, for the participants that were not familiar with OWL, the requirements that had the highest percentage of correct answers were those related to instances or related both to a relation and a hierarchy between terms. For the participants that were familiar with OWL, the requirements related to disjoint classes had the highest percentage of correct answers. Finally, for the participants that were experts, the requirements that had the highest percentage of correct answers were those related to disjoint classes and to relations between terms, and those requirements that had more than one restriction such as a relation and a hierarchy or union between classes.

Concerning incorrect results, in the case of Themis, the requirements with the lowest percentage of correct answers for the participants that were not familiar with OWL were those related to hierarchies and to a relation and union between terms. Regarding the participants that were familiar with the OWL language, those requirements related to a relation and a hierarchy had the lowest percentage of correct answers, although it is higher than 50\%. Finally, regarding experts in OWL, the requirements with the lowest percentage of correct answers were those related to a hierarchy and disjoint classes.

Table 7.8: Percentage of results for Themis grouped by type of requirement

\begin{tabular}{|l|r|r|r|r|r|r|r|r|r|}
\cline { 2 - 11 } \multicolumn{1}{c|}{} & \multicolumn{9}{c|}{ Participants } \\
\hline \multirow{2}{*}{ Requirement } & \multicolumn{2}{c|}{ Not familiar } & \multicolumn{3}{c|}{ Familiar } & \multicolumn{3}{c|}{ Expert } \\
\cline { 2 - 11 } & $\mathbf{C}$ & $\mathbf{I}$ & $\mathbf{U}$ & $\mathbf{C}$ & $\mathbf{I}$ & $\mathbf{U}$ & $\mathbf{C}$ & $\mathbf{I}$ & $\mathbf{U}$ \\
\hline Hierachy & $0 \%$ & $100 \%$ & $0 \%$ & $77 \%$ & $23 \%$ & $0 \%$ & $62 \%$ & $37 \%$ & $0 \%$ \\
\hline Disjointness & $50 \%$ & $50 \%$ & $0 \%$ & $100 \%$ & $0 \%$ & $0 \%$ & $100 \%$ & $0 \%$ & $0 \%$ \\
\hline Terms relation & $33 \%$ & $67 \%$ & $0 \%$ & $81 \%$ & $17 \%$ & $2 \%$ & $100 \%$ & $0 \%$ & $0 \%$ \\
\hline Instance & $100 \%$ & $0 \%$ & $0 \%$ & $62 \%$ & $33 \%$ & $5 \%$ & $80 \%$ & $20 \%$ & $0 \%$ \\
\hline Cardinality & $67 \%$ & $33 \%$ & $0 \%$ & $76 \%$ & $16 \%$ & $86 \%$ & $90 \%$ & $10 \%$ & $0 \%$ \\
\hline $\begin{array}{l}\text { Terms relation and } \\
\text { cardinality }\end{array}$ & - & - & - & $86 \%$ & $0 \%$ & $14 \%$ & $75 \%$ & $25 \%$ & $0 \%$ \\
\hline $\begin{array}{l}\text { Hierarchy and } \\
\text { disjointness }\end{array}$ & - & - & - & $86 \%$ & $14 \%$ & $0 \%$ & $50 \%$ & $50 \%$ & $0 \%$ \\
\hline $\begin{array}{l}\text { Terms relation and } \\
\text { hierarchy }\end{array}$ & $100 \%$ & $0 \%$ & $0 \%$ & $57 \%$ & $14 \%$ & $0 \%$ & $100 \%$ & $0 \%$ & $0 \%$ \\
\hline Terms relation and union & $0 \%$ & $100 \%$ & $0 \%$ & $71 \%$ & $0 \%$ & $26 \%$ & $100 \%$ & $0 \%$ & $0 \%$ \\
\hline
\end{tabular}

The results retrieved from TDDOnto2 regarding the percentage of correct answers grouped by types of requirements differ from the results retrieved from Themis. In this 
case, regarding the participants that were not familiar with OWL, the highest percentage of correct answers were related to instances and cardinalities. Concerning participants that were familiar with the OWL language, the requirements related to hierarchies and the requirements that define both hierarchies and disjoint classes were the ones with the highest percentage, although they did not reach 100\% of correct answers. Finally, concerning experts, the requirements related to instances, as well as those related to both relation and hierarchies, had $100 \%$ of correct answers.

Concerning incorrect results, for TDDOnto2 the requirements with the lowest percentage of correct results for all the participants were those related to several restrictions. For participants that were not familiar with OWL, the requirements related to a relation and cardinality between terms, to a relation between terms and a hierarchy, and to a relation and union between terms, had $0 \%$ of correct answers. Regarding participants that were familiar with OWL, the requirements related to a relation and union between terms had the lowest percentage of correct answers. Finally, for those participants experts in OWL the requirements related to a relation and cardinality between terms, to a relation between terms and disjointness, and to a relation and union between terms had $67 \%$ of correct answers, which was the lowest percentage of correct answers for this type of participants.

Table 7.9: Percentage of results for TDDOnto2 grouped by type of requirement

\begin{tabular}{|l|r|r|r|r|r|c|c|c|c|}
\cline { 2 - 12 } \multicolumn{1}{c|}{} & \multicolumn{9}{c|}{ Participants } \\
\hline \multirow{2}{*}{\multicolumn{1}{c|}{ Requirement }} & \multicolumn{2}{c|}{ Not familiar } & \multicolumn{3}{c|}{ Familiar } & \multicolumn{3}{c|}{ Expert } \\
\cline { 2 - 12 } & $\mathbf{C}$ & $\mathbf{I}$ & $\mathbf{U}$ & $\mathbf{C}$ & $\mathbf{I}$ & $\mathbf{U}$ & $\mathbf{C}$ & $\mathbf{I}$ & $\mathbf{U}$ \\
\hline Hierachy & $80 \%$ & $0 \%$ & $20 \%$ & $86 \%$ & $11 \%$ & $4 \%$ & $93 \%$ & $7 \%$ & $0 \%$ \\
\hline Disjointness & $75 \%$ & $25 \%$ & $0 \%$ & $64 \%$ & $36 \%$ & $0 \%$ & $75 \%$ & $25 \%$ & $0 \%$ \\
\hline Terms relation & $57 \%$ & $0 \%$ & $43 \%$ & $72 \%$ & $25 \%$ & $2 \%$ & $76 \%$ & $24 \%$ & $0 \%$ \\
\hline Instance & $100 \%$ & $0 \%$ & $0 \%$ & $73 \%$ & $27 \%$ & $0 \%$ & $100 \%$ & $0 \%$ & $0 \%$ \\
\hline Cardinality & $100 \%$ & $0 \%$ & $0 \%$ & $82 \%$ & $18 \%$ & $0 \%$ & $95 \%$ & $5 \%$ & $0 \%$ \\
\hline $\begin{array}{l}\text { Terms relation and } \\
\text { cardinality }\end{array}$ & $0 \%$ & $0 \%$ & $100 \%$ & $58 \%$ & $0 \%$ & $42 \%$ & $67 \%$ & $33 \%$ & $0 \%$ \\
\hline $\begin{array}{l}\text { Hierarchy and } \\
\text { disjointness }\end{array}$ & $100 \%$ & $0 \%$ & $0 \%$ & $83 \%$ & $17 \%$ & $0 \%$ & $67 \%$ & $33 \%$ & $0 \%$ \\
\hline $\begin{array}{l}\text { Terms relation and } \\
\text { hierarchy }\end{array}$ & $0 \%$ & $0 \%$ & $100 \%$ & $60 \%$ & $40 \%$ & $0 \%$ & $100 \%$ & $0 \%$ & $0 \%$ \\
\hline Terms relation and union & $0 \%$ & $0 \%$ & $100 \%$ & $17 \%$ & $0 \%$ & $83 \%$ & $67 \%$ & $33 \%$ & $0 \%$ \\
\hline
\end{tabular}

For Protégé, the type of requirement with the highest percentage of correct answers for the all the participants was the one related to a relation and union between terms, which was not a trivial requirement for participants using the other tools. This might 
happen because of the visualisation of restrictions provided by Protégé.

The requirements related to hierarchies in Protégé had a low percentage of correct answers compared with the other tools. For participants that are familiar with OWL only $33 \%$ of this type of requirements had correct answers, while for experts only 40\%. This situation might stem because Protége only shows the direct superclass and, consequently, users cannot see the entire hierarchy unless they navigate through the classes. This might also happen with those requirements related to disjoint classes and to relations between terms.

Table 7.10: Percentage of results for Protégé grouped by type of requirement

\begin{tabular}{|l|c|c|c|c|c|c|c|c|c|}
\cline { 2 - 10 } \multicolumn{1}{c|}{} & \multicolumn{9}{c|}{ Participants } \\
\hline \multirow{2}{*}{\multicolumn{1}{c|}{ Requirement }} & \multicolumn{1}{c|}{ Not familiar } & \multicolumn{2}{c|}{ Familiar } & \multicolumn{3}{c|}{ Expert } \\
\cline { 2 - 11 } & C & I & U & C & I & U & C & I & U \\
\hline Hierachy & $53 \%$ & $47 \%$ & $0 \%$ & $33 \%$ & $67 \%$ & $0 \%$ & $40 \%$ & $60 \%$ & $0 \%$ \\
\hline Disjointness & $75 \%$ & $25 \%$ & $0 \%$ & $25 \%$ & $75 \%$ & $0 \%$ & $50 \%$ & $50 \%$ & $0 \%$ \\
\hline Terms relation & $64 \%$ & $36 \%$ & $0 \%$ & $43 \%$ & $57 \%$ & $0 \%$ & $14 \%$ & $86 \%$ & $0 \%$ \\
\hline Instance & $67 \%$ & $25 \%$ & $8 \%$ & $50 \%$ & $50 \%$ & $0 \%$ & $50 \%$ & $50 \%$ & $0 \%$ \\
\hline Cardinality & $67 \%$ & $33 \%$ & $0 \%$ & $52 \%$ & $48 \%$ & $0 \%$ & $86 \%$ & $14 \%$ & $0 \%$ \\
\hline $\begin{array}{l}\text { Terms relation and } \\
\text { cardinality }\end{array}$ & $75 \%$ & $25 \%$ & $0 \%$ & $33 \%$ & $67 \%$ & $0 \%$ & $50 \%$ & $50 \%$ & $0 \%$ \\
\hline $\begin{array}{l}\text { Hierarchy and } \\
\text { disjointness }\end{array}$ & $67 \%$ & $33 \%$ & $0 \%$ & $67 \%$ & $33 \%$ & $0 \%$ & $100 \%$ & $0 \%$ & $0 \%$ \\
\hline $\begin{array}{l}\text { Terms relation and } \\
\text { hierarchy }\end{array}$ & $60 \%$ & $40 \%$ & $0 \%$ & $33 \%$ & $67 \%$ & $0 \%$ & $0 \%$ & $100 \%$ & $0 \%$ \\
\hline Terms relation and union & $83 \%$ & $17 \%$ & $0 \%$ & $100 \%$ & $0 \%$ & $0 \%$ & $100 \%$ & $0 \%$ & $0 \%$ \\
\hline
\end{tabular}

Table 7.11 summarises the results obtained per type of requirement grouped by tool, as well as the total percentage of results for all tools. With the information depicted in Table 7.11, it is confirmed that the analysed tools had more than $50 \%$ of correct results on average for all the types of requirements. Moreover, it is also confirmed that, on average, the requirements more complex to be verified, i.e., with the worst percentage of incorrect results, were those related to terms relations and hierarchies.

It must also be remarked that the variability in the results for the different tools is high. This makes sense because the testing approaches provided by each tool facilitate dealing with certain types of tests above others. For example, the percentage of incorrect results regarding disjointness is quite low for Themis, since the tool only requires writing a simple test expression; on the other side, checking disjointness with Protégé requires browsing the disjointness axioms in the ontology and even running a reasoner when those axioms are not explicit. 


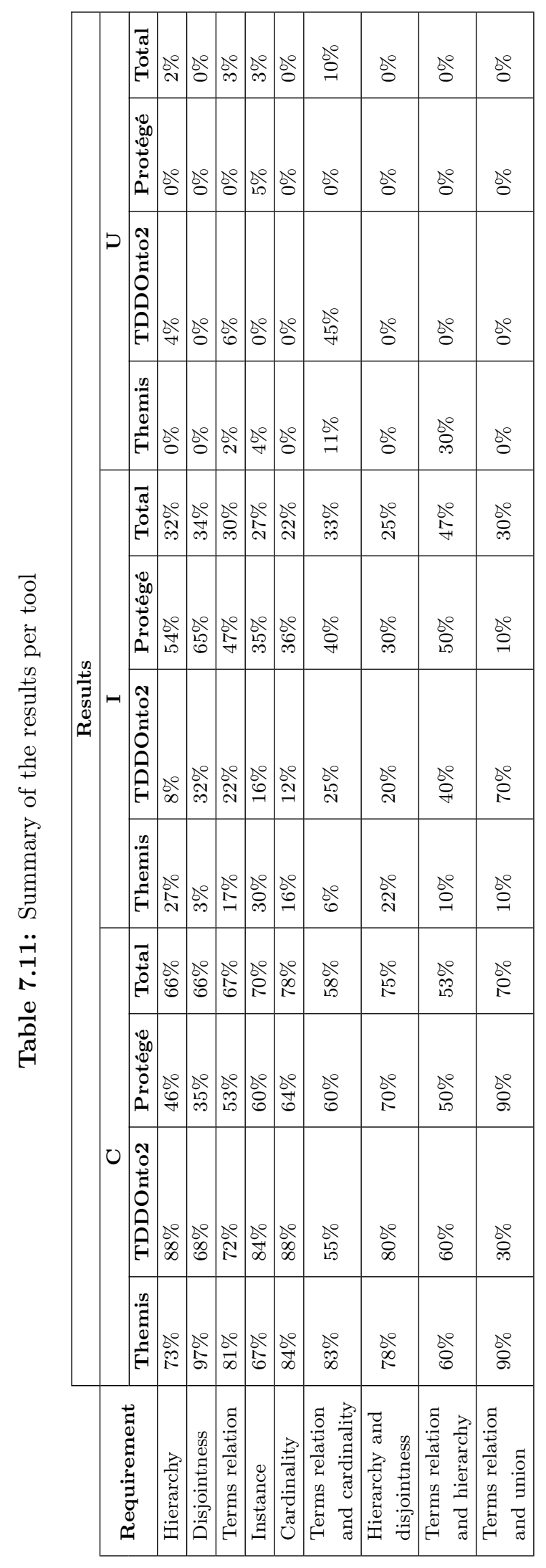




\subsubsection{Usefulness, satisfaction and ease of use analysis}

After the empirical analysis, information collected though the USE questionnaire included in Annex C was analysed. To this end, 30 questionnaires (10 questionnaires for each tool) were collected from participants. In Figures 7.4 7.5 and 7.6 an overview of the results is provided.

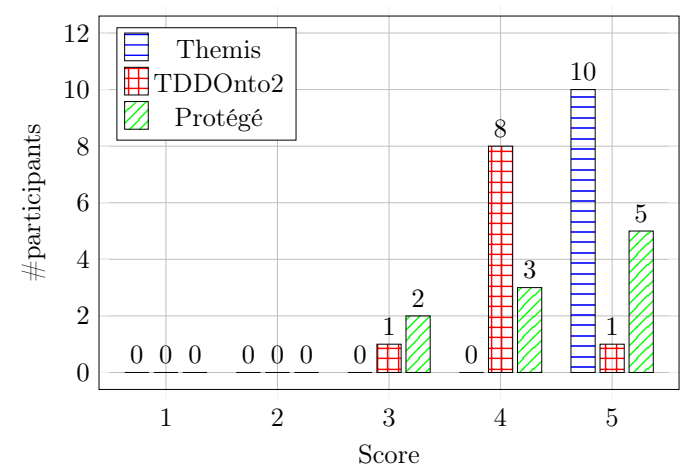

(a) It is useful

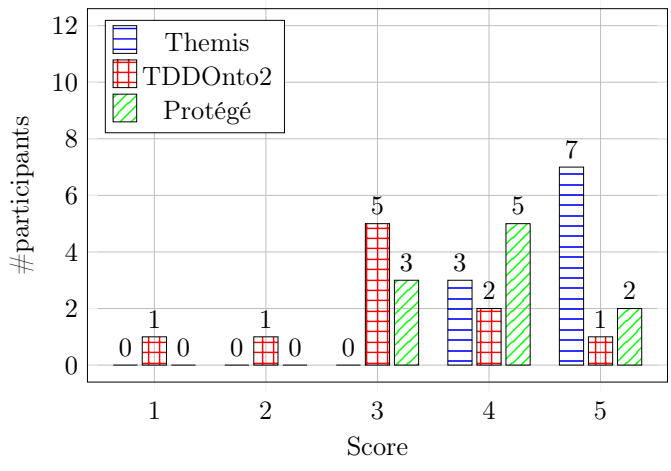

(b) It makes the things I want to accomplish easier to get done

Figure 7.4: Summary of the usefulness results

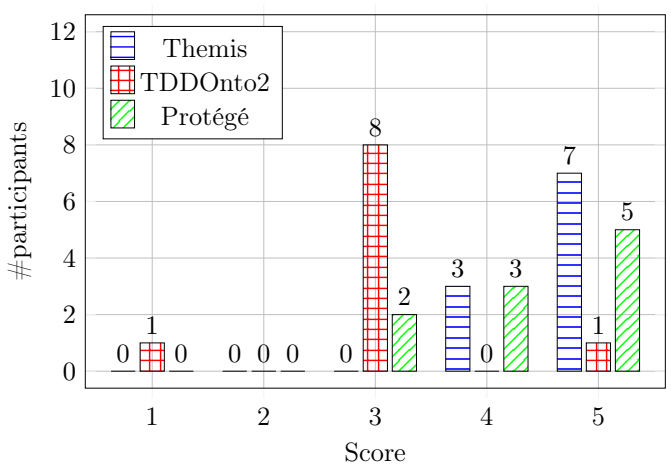

(a) I am satisfied with it

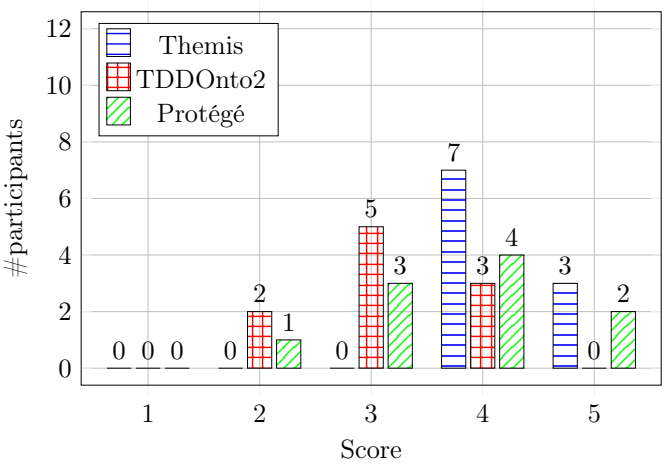

(b) It works the way I want it to work

Figure 7.5: Summary of the satisfaction results

Figures 7.4, 7.5 and 7.6 show that regarding usefulness, satisfaction and usability, Themis had the best results, followed by Protégé and by TDDOnto2. However, although Protégé had good results in the questionnaire, especially as an easy to use and intuitive tool, it leads to worse results in the verification process. From the analysis and feedback from participants it was also found that for those participants without any knowledge related to the OWL language and ontologies, learning a syntax for generating tests was 
too complex. In that scenario, Protégé is the most useful and usable tool. However, it was also found that these participants are able to search for classes and properties in an ontology, but they cannot verify other types of requirements, since it is difficult for them to understand the different types of restrictions that can be defined in an ontology. Moreover, instead of having complex test expressions, or concatenated axioms in the case of TDDOnto2, it was easier and more intuitive for the participants to use simple tests, even though they had to split a test into several sub-tests. However, in general, all participants found useful these tools for verifying ontologies.

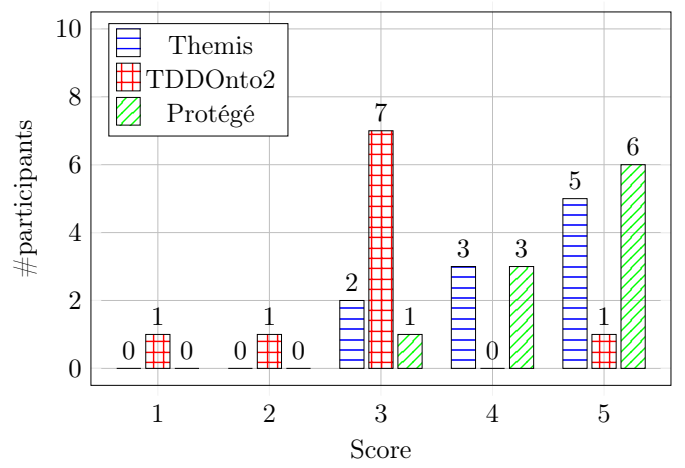

(a) It is easy to use

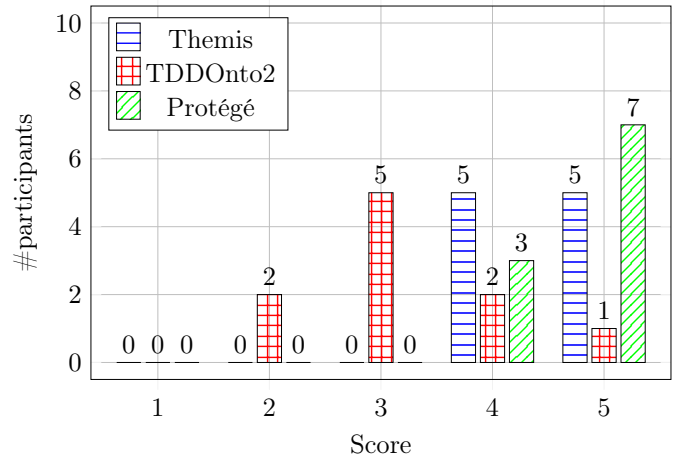

(c) It is easy to remember how to use it

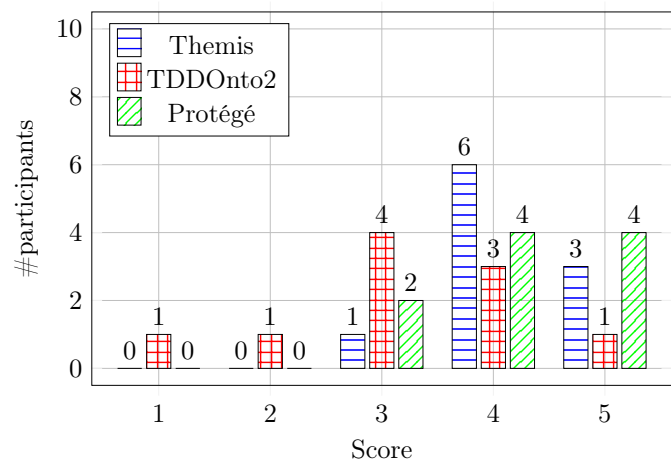

(b) It is user friendly

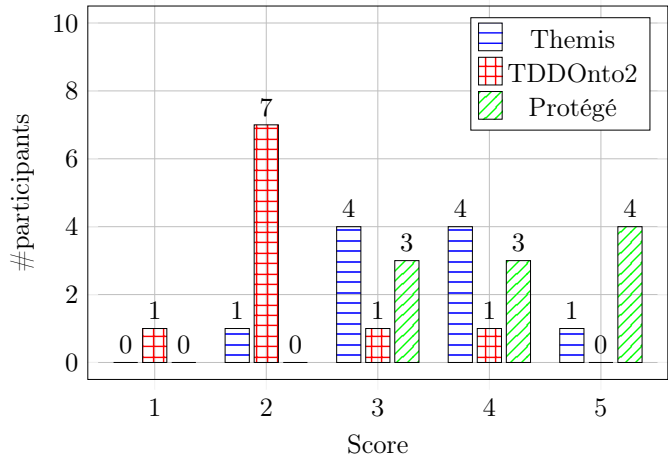

(d) Using it is effortless

Figure 7.6: Summary of the usability results 


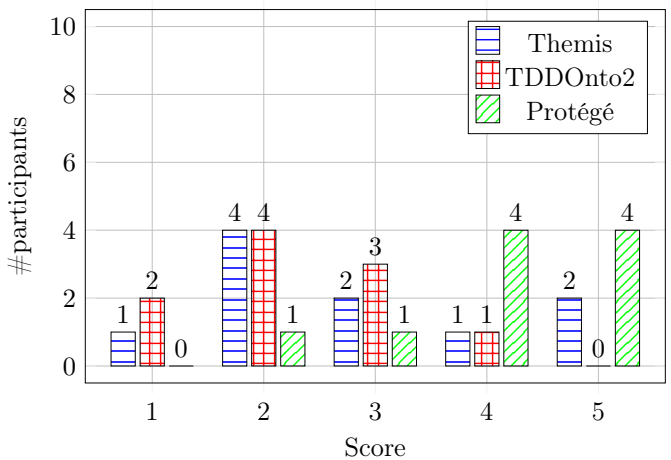

(e) I can use it without written instructions

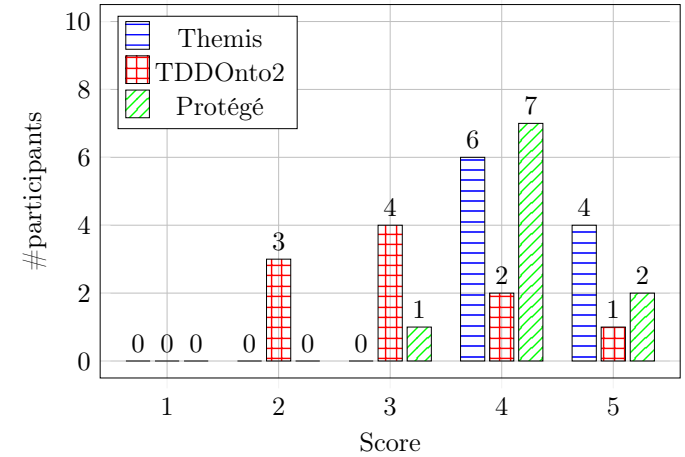

(f) I do not notice any inconsistency as a use it

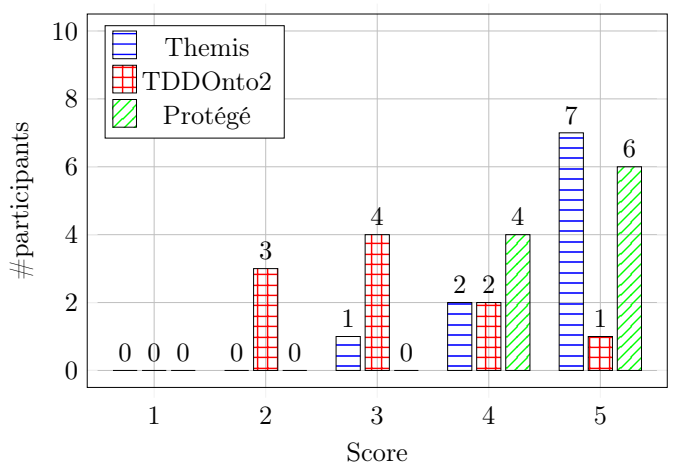

(g) It is easy to learn to use it

Figure 7.6: Summary of the usability results (Cont.)

In addition to the comparative of the tools, along these questionnaires some strengths of Themis were explicitly pointed out by users as 44. "Very useful tool", "Easy to use", "The autocomplete makes it very easy", "The fact that it is a web application is very rewarding, as I won't need to download prerequisites, update the java version, etc.".

However, the richest side of the provided feedback is the set of proposals to improve the tool. The most important feedback in this regard refers to the following issues:

- To improve the syntax checking to help users to define the tests.

- To allow to test local ontologies not published online.

- To improve autocompletion in order to distinguish classes, instances and properties.

- To recommend possible tests based on a requirement written in natural language.

\footnotetext{
${ }^{44}$ The following comments have been taken literally from the feedback questionnaires
} 
Based on this feedback and as already mentioned in Section 4.4, Themis includes a syntax checker that indicates the user whether the test is syntactically correct or not. Moreover, it allows loading local ontologies in order to test ontologies that are not available online. Further software extensions are planned to: (a) improve autocompletion to clarify whether a test should include an ontology class, instance or property, and (b) to help users to detect which test is needed to check a given requirement.

\subsubsection{Conclusions of the experiment}

With respect to the hypothesis, the following conclusions can be outlined:

- H1. Using a testing language for defining tests based on functional requirements facilitates the ontology testing process regarding the reduction of errors in users that are familiar and expert in OWL.

Themis and TDDOnto2, both based on a testing language, had a significantly higher percentage of correct results in comparison with Protégé for users that are familiar and expert in the OWL language. Moreover, although both tools have a learning curve in terms of the time spent per requirement, they had a lower number of incorrect results for these types of users. Therefore, this hypothesis is true.

\subsection{Evaluation of the metrics derived from the verification process}

Chapter 5 presented different metrics associated to three artefacts generated and used during the ontology verification process, namely, the Ontology Requirements Specification Document (ORSD), the Ontology Requirements Test Suite (ORTS) and the ontology implementation. The combination of the information provided by such metrics aims at providing information related to the development process. In this evaluation, those metrics were applied to a set of different ontology development processes and the retrieved data was collected and analysed, in order to check the influence of some metrics on others, with the aim of obtaining more information related to those development processes. The following hypothesis is made for this evaluation (Section 3.3):

- H2. There is a direct correlation between some metrics related to functional requirements and those metrics related to the implementation and the tests of the ontology. 


\subsubsection{Experimental design}

The goal of this evaluation was to analyse the influence of the artefacts generated and used during the verification process, i.e., the ORSD, the ORTS and the ontology implementation, on the others. To that end, a set of ontology development processes was collected and the metrics presented in Chapter 5, which are related to the three artefacts, were calculated. Afterwards, it was analysed whether some metrics influence others; these metrics determine different characteristics related to the artefacts, such as the complexity of each requirement, the percentage of changed requirements and the number of tested terms.

In order to perform the experiment, the three aforementioned artefacts must be generated. For the generation of the ORSD, the goal of the ontology, the domain that the ontology should model, and technical details of the ontology like the implementation language, among other requirements, must be collected. Concerning the generation of the ORTS, the set of tests to verify the requirements included in the ORSD must be defined. These tests can be written following the testing method described in Chapter 4 . where the tests are written following a testing language. Finally, to obtain the ontology implementation, ontology engineers have to encode the ontology using an implementation language, for example, OWL.

The metrics used in the experiment included those base product metrics related to the requirements, the tests and the ontology implementation, i.e., NReq, NA4 NTests, as well as calculated product metrics, i.e., ReqACo, CovReqPtc and TestEffRatio. This experiment also used process metrics related to the development time and volatility, i.e., VReqTime, ReqVolatility and OVolatility. These metrics are described in Section 5.4 .

This experiment was performed by analysing the following aspects of the ORSD and the ORTS and how they influence the rest of the artefacts. Figure 7.7 shows these aspects together with the metrics involved in the analysis:

- The number of requirements $(N R e q)$ included in the ORSD and how it influences the number of tests (NTests), the requirement development time (VReqTime), the size of the ontology $(N A)$, and the expressivity of the ontology.

- The complexity of requirements (ReqACo) included in the ORSD and how it influences the number of tests (NTests), the requirement development time (VReqTime), and the size of the ontology $(N A)$.

\footnotetext{
${ }^{45}$ This metric is defined in Protégé.
} 
- The volatility of the requirements (ReqVolatility) included in the ORSD and how it influences the volatility of the ontology (OVolatility).

- The number of tests (NTests) included in the ORTS and how it influences the tested terms (TestEffRatio) in the ontology.

- The tested terms (TestEffRatio) included in the ORTS and how they influence the number of requirements satisfied by the ontology (CovReqPtc).

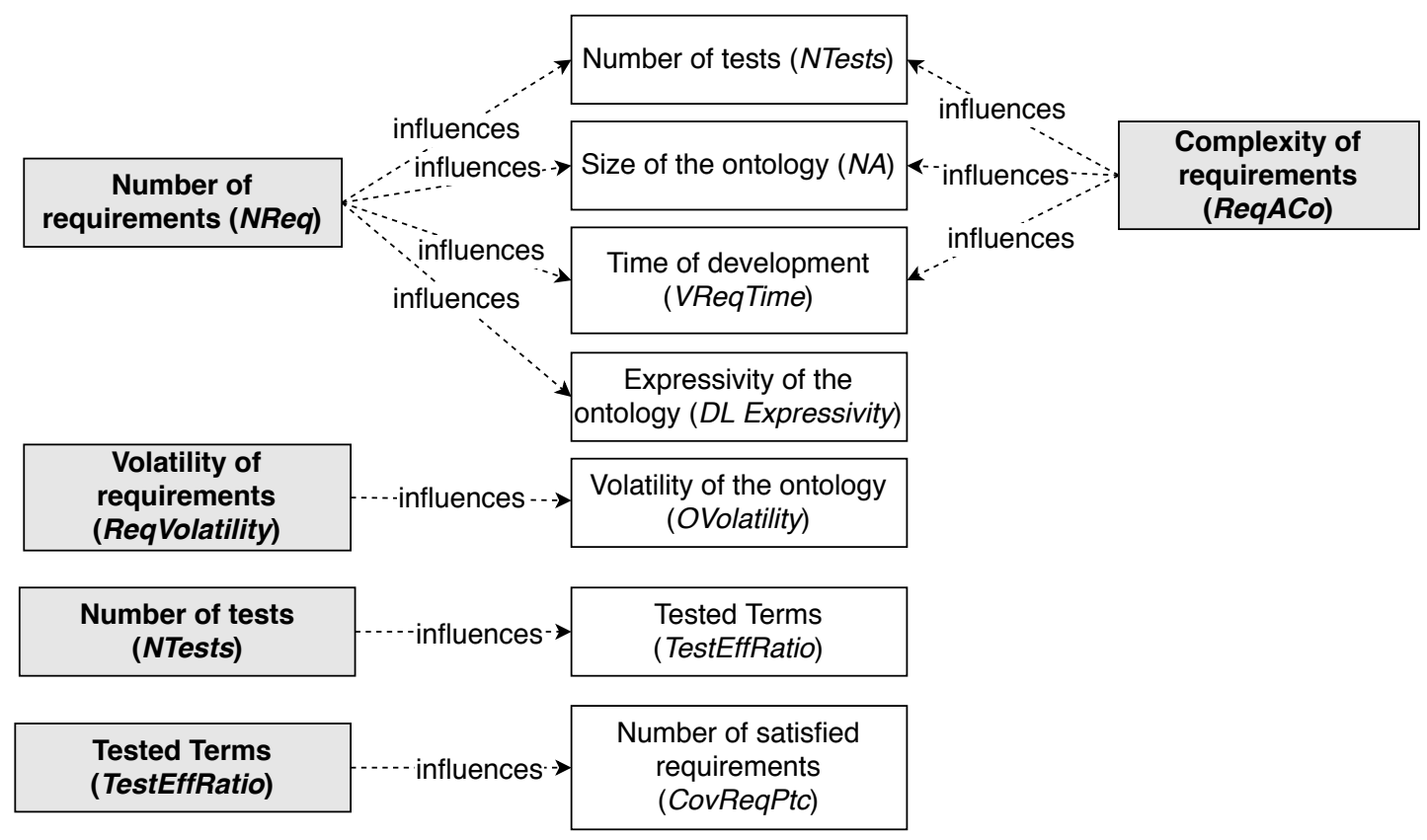

Figure 7.7: Aspects to be analysed and their associated metrics

To check the influences of these metrics extracted from the three artefacts, the correlation coefficient (Basilevsky, 2009) between the metrics related to the ORSD, the ORTS and ontology implementation was computed. This coefficient allows determining the strength of the association between the analysed metrics. The formula used to calculate such coefficient is the following:

$$
\rho(X, Y)=\frac{\operatorname{Cov}(X, Y)}{\sqrt{\operatorname{Var}(X) \operatorname{Var}(Y)}}
$$

The correlation coefficient has a value between -1 and 1 , where 1 is total positive linear correlation, 0 is no linear correlation, and -1 is total negative linear correlation. In this case, the relationship between two variables will be considered strong when the 
correlation coefficient value is greater than 0.6 (positive correlation) or lower than -0.6 (negative correlation).

\subsubsection{Results and discussion}

In this experiment the ontology metrics were applied to the development process of the ontology network developed in the European VICINITY project, which includes five ontologies, i.e., the VICINITY Core (Core) ${ }^{46}$ the Web of Things (WoT) ${ }^{47}$ the WoT mappings (Mappings) ${ }^{48}$ the Adapters (Adapters) ${ }^{49}$ and the Datatypes (Datatypes) ontologies 50

In this particular case, the ORSD associated to each ontology stores the requirements that were identified by domain experts and written in the form of competency questions and statements. They were stored in online spreadsheets to facilitate sharing, edition and version control. Concerning the ORTSs, the identified requirements were formalised into test cases in order to verify them following the testing process described in Section 4.2. Finally, each ontology was encoded using the OWL language. For each of the ontologies, its ORSD is available in the VICINITY ontology portal 51 Moreover, the ontology implementation and the ORTS were stored in a GitHub repository which, due to its version control, allows the ontology engineers to be aware of the evolution of the artefacts during the development iterations 52

The following subsections describe the results obtained after gathering the metrics from the ontologies and analysing the influences between them. These sections are organised according to the aspects that were analysed to check the influences derived of different metrics, namely, the number of requirements, the complexity of requirements, the volatility of requirements, the number of tests, and the tested terms.

\subsubsection{Influence derived from the number of requirements}

Ontology requirements can provide team leaders and developers with insights about the development process, since they represent the knowledge that should be added to an ontology. The number of requirements (NReq) metric was used to determine the total

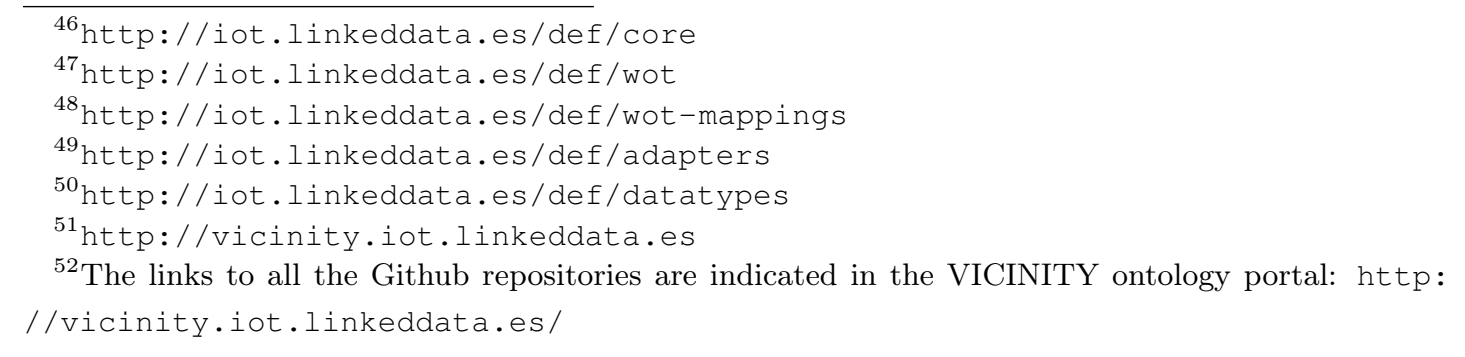


number of requirements defined for the five analysed ontologies in each sprint, which are summarised in Table 7.12 ,

To analyse the possible influences of the number of requirements in the development process, this metric was compared with the number of defined tests (NTests), the development time (VDevTime), the size $(N A)$ and the DL expressivity of the five ontologies.

Table 7.12: Number of requirements and tests in each sprint ( $\mathrm{R}$ refers to Requirements and $\mathrm{T}$ refers to Tests)

\begin{tabular}{|c|c|c|c|c|c|c|c|c|c|c|c|c|}
\hline & \multicolumn{2}{|c|}{ Sprint 1} & \multicolumn{2}{|c|}{ Sprint 2} & \multicolumn{2}{|c|}{ Sprint 3} & \multicolumn{2}{|c|}{ Sprint 4} & \multicolumn{2}{|c|}{ Sprint 5} & \multicolumn{2}{|c|}{ Sprint 6} \\
\hline & $\# \mathbf{R}$ & $\# \mathbf{T}$ & $\# \mathbf{R}$ & $\# \mathbf{T}$ & $\# \mathbf{R}$ & $\# \mathbf{T}$ & $\# \mathbf{R}$ & $\# \mathbf{T}$ & $\# \mathbf{R}$ & $\# \mathbf{T}$ & $\# \mathbf{R}$ & $\# \mathbf{T}$ \\
\hline Core & 116 & 116 & 153 & 153 & 156 & 156 & 154 & 154 & 173 & 173 & 67 & 67 \\
\hline WoT & 33 & 33 & 32 & 32 & 24 & 24 & 14 & 17 & - & - & - & - \\
\hline Mappings & 13 & 13 & 15 & 15 & - & - & - & - & - & - & - & - \\
\hline Adapters & - & - & - & - & - & - & - & - & - & - & 171 & 171 \\
\hline Datatypes & - & - & - & - & - & - & - & - & - & - & 11 & 14 \\
\hline
\end{tabular}

Influence of the number of requirements on the number of tests. First, the number of tests defined for each ontology (NTests) was determined (also summarised in Table 7.12). For the five ontologies, tests were generated to verify the requirements and, therefore, for the majority of ontologies there is the same number of requirements and tests. Moreover, in those cases where the number of requirements does not coincide with the number of tests, the latter is higher; although there was defined at least one test per requirement, some requirements needed more than one test to be verified.

Furthermore, the correlation coefficient between these two metrics is 0.99. Figure 7.8 shows the scatterplot associated to NReqs and NTests metrics, which shows a strong relationship between these two metrics.

Influence of the number of requirements on the ontology size. The size of the ontologies $(N A)$ during the sprints was also calculated in order to be compared with the number of requirements. Figures 7.9 and 7.10 illustrate the evolution of the size of the ontologies during their development process. The former indicates the $N A$ without considering the imported ontologies, while the latter indicates the $N A$ including the imported ontologies.

Figure 7.9 shows that while the size of the WoT and the Mappings ontologies have small variations, the size of the Core ontology increases from sprint 2 onwards. This is due to the fact that the Core ontology, considering that several domain experts from 


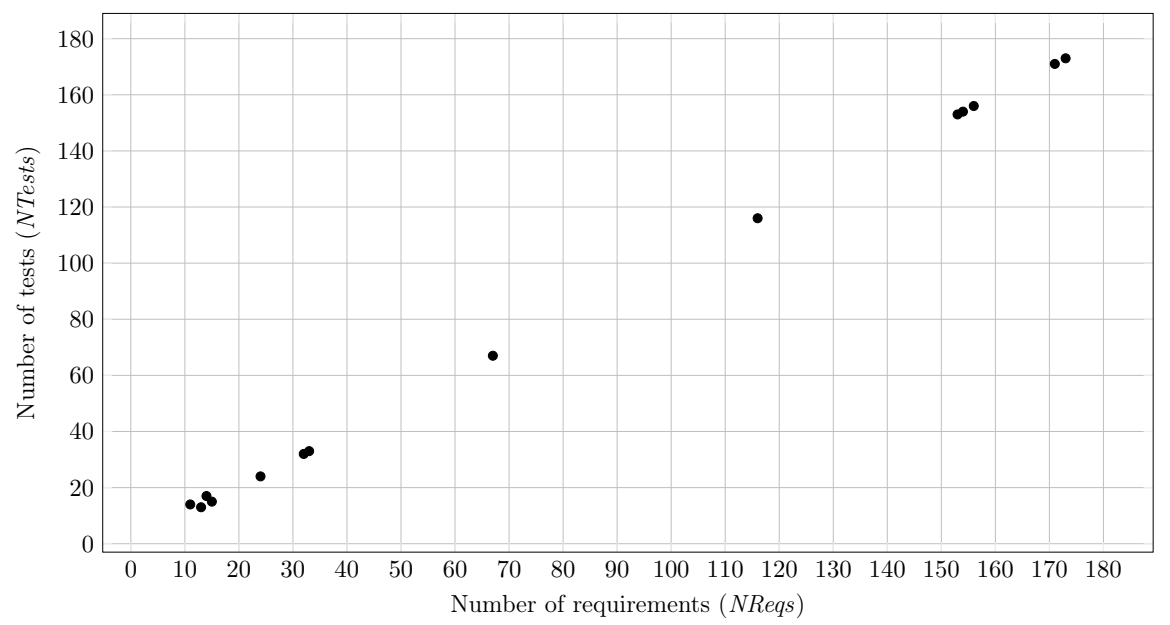

Figure 7.8: Scatterplot related to NReqs and NTests

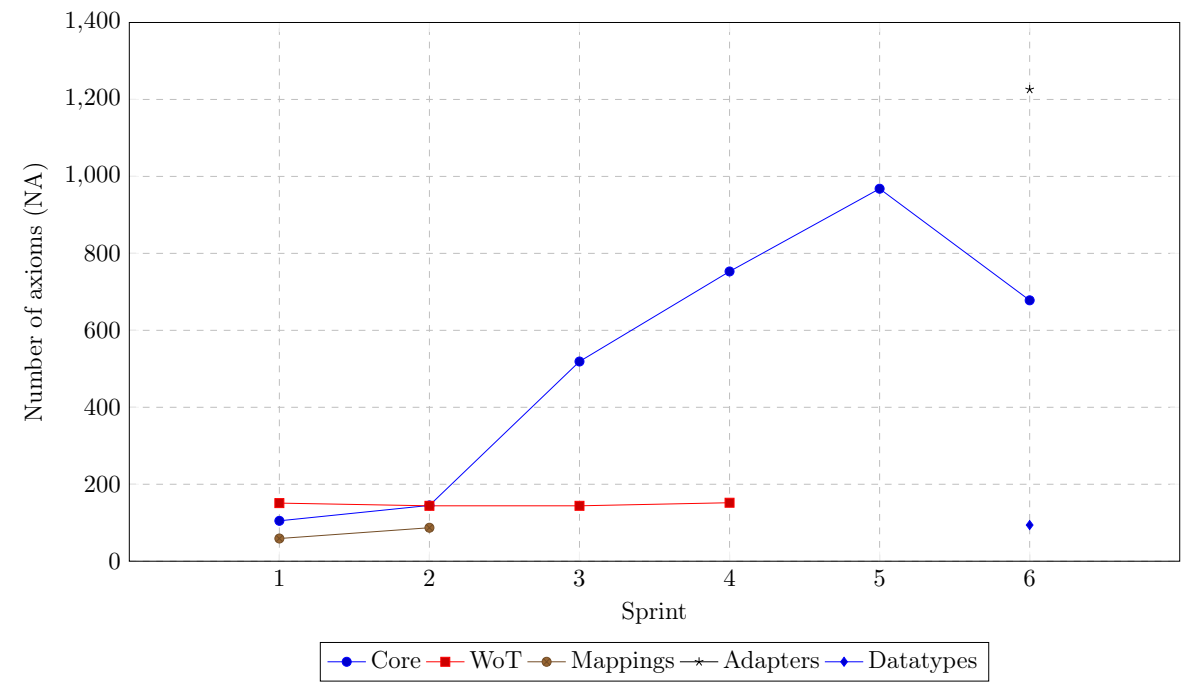

Figure 7.9: Size of the ontologies during their development process not including imported ontologies

different domains were involved in the development process, receives more requirements than the other ontologies during the sprints. Moreover, in the last sprint the ontology that has more defined requirements is the Adapters ontology, which is also the largest ontology.

With the information obtained from the values presented in Figure 7.9 and Table 7.12, the correlation coefficient is 0.73 . Figure 7.11 depicts the scatterplot associated to the NReqs and NA metrics, which shows a relationship between these two metrics. 




Figure 7.10: Size of the ontologies during their development process including imported ontologies

Therefore, the number of requirements affects the size of ontologies. It should be mentioned that in this situation the imported ontologies are not included in the $N A$ metric, since only the axioms that were added by the developers involved in the project were taken into account.

Nevertheless, Figure 7.10 illustrates the size the ontologies $(N A)$ considering their imports. In this figure it can be observed that the Mappings ontology has increased its size, since it imports several ontologies (due to the needs of the application using it) even though it is not indicated in the requirements. The evolution of the rest of the ontologies remains similar, and the Adapters ontology is still the largest one.

\section{Influence of the number of requirements on the requirement development}

time. The number of requirements was also compared with the requirement development time (VReqTime). To calculate the development time spent in the five mentioned ontologies and to check whether the number of requirements influences such development times, the information about the commits done by the ontology engineers in the GitHub repositories and the information stored in the spreadsheets with the ontology requirements were retrieved. Figure 7.12 shows the average time spent per requirement and sprint (VReqTime) for each ontology version. 




Figure 7.11: Scatterplot related to NReqs and NA (without imports)

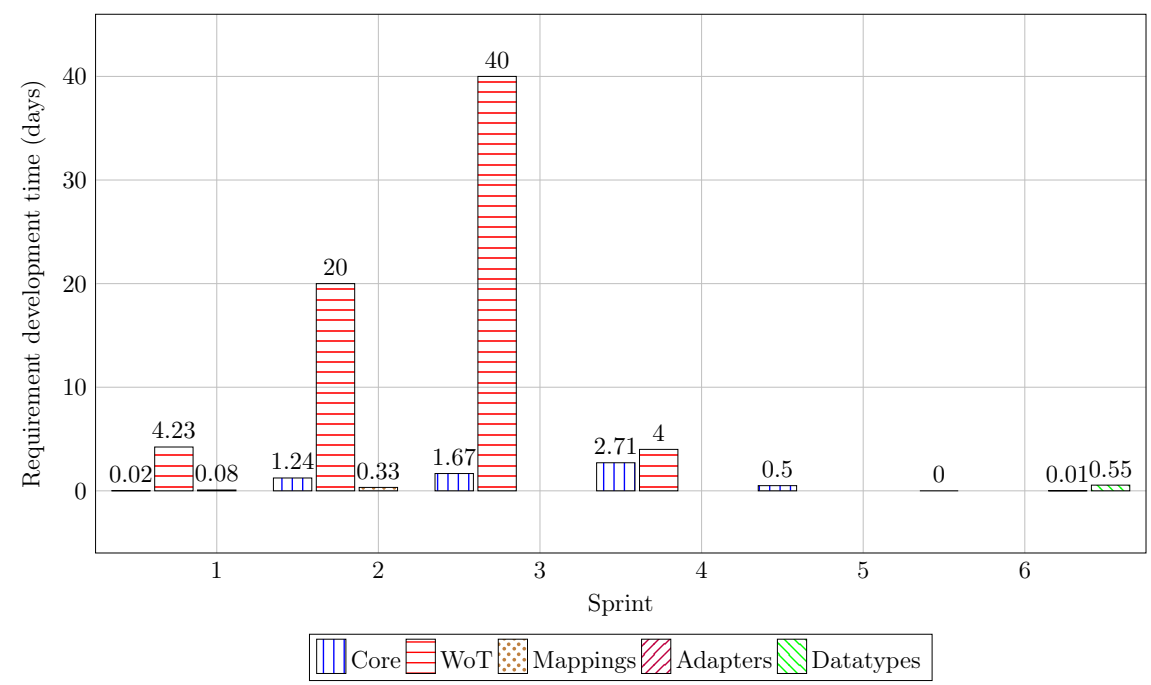

Figure 7.12: Requirement development average time for each ontology version

For the five ontologies, the developers only used the master branch of the GitHub repository to develop the ontology and the online spreadsheets store the modifications done over the ORSD during the development sprints. Therefore, each sprint starts when the spreadsheet is modified to indicate which are the requirements planned for the sprint and ends with the commit that releases a new version of the ontology. Due to the fact that these software tools only allow calculating the spent time in days, the precision of the results is not as accurate as it would be if it had been calculated in hours. 
From the comparison between the values presented in Table 7.12 and Figure 7.12 , it can be observed that the number of requirements does not influence directly the time of development. The ontology engineers implemented 171 requirements for the Adapters ontology in only one sprint, while for the 33 initial requirements of the WoT ontology they needed 4 sprints. The reason for this is that the requirements defined for the Adapters ontology were easier to implement or they were already implemented in other ontology, as already mentioned, due to the fact that part of the Adapters ontology was taken from the Core module.

With the gathered information related to the number of requirements and the development time shown in Table 7.12 and Figure 7.12 , the correlation coefficient between these two metrics is -0.33 , which confirms that the number of requirements does not influence directly the time of development.

Influence of the number of requirements on the ontology expressivity. Finally, the DL expressivity (Baader et al. 2008) of the ontologies during the sprints was calculated and is summarised in Table 7.13. The Mappings ontology, which has only 13 and 15 requirements in sprint 1 and 2, has the same expressivity as Adapters, which has 171 requirements. This occurs because the Mappings ontology imports, among others, the Core ontology, increasing its DL expressivity.

Moreover, the correlation coefficient between these two metrics is 0.53 , which was calculated by associating to each DL expressivity a number that indicates the degree of expressiveness from less expressive to higher, e.g., ALF expressiveness is associated to 1. This correlation coefficient concluded that there might be a relationship between the number of requirements and the expressivity of the ontology, although not enough to be considered significant in this use case.

Table 7.13: DL expressivity of the ontologies during each sprint

\begin{tabular}{|l|l|l|l|l|c|c|}
\cline { 2 - 7 } \multicolumn{1}{c|}{} & Sprint 1 & Sprint 2 & Sprint 3 & Sprint 4 & Sprint 5 & Sprint 6 \\
\hline Core & SRIF(D) & SRIF(D) & SRIF(D) & SRIF(D) & SRIQ(D) & SRIQ(D) \\
\hline WoT & ALCHIF(D) & ALCHIF(D) & ALCHIF(D) & ALCHIF(D) & - & - \\
\hline Mappings & SRIQ(D) & SRIQ(D) & - & - & - & - \\
\hline Adapters & - & - & - & - & - & SRIQ(D) \\
\hline Datatypes & - & - & - & - & - & ALF(D) \\
\hline
\end{tabular}

\subsubsection{Influences derived from requirements complexity}

The analysis of the complexity of requirements may help team leaders along with ontology engineers in estimating the effort needed to implement a requirement. The more 
complex a requirement is, the more complex may be to implement it in the ontology. The Requirements Axiom Complexity (ReqACo) metric was used to obtain the requirements complexity for the five ontologies, due to the fact that it measures the axioms needed to implement each requirement. The number of axioms was obtained by counting the number of axioms to be added to the corresponding ontology for each requirement $(N A)$.

Figure 7.13 shows the distribution of the requirements axiom complexity in the last sprint of the five analysed ontologies, where the requirements are up to date and, as a consequence, are valuable to make effort estimations. For the sake of clarity, the graphs in Figure 7.13 consider a maximum of $50 \%$ ReqACo, which represents that the requirement includes 11 axioms out of the 22 considered in the metric as stated in Section 5.4. As an example of the information that can be retrieved from Figure 7.13, the figure shows that the Mappings ontology has 12 requirements with a ReqACo between $15 \%$ and $20 \%$ (e.g., 4 axioms out of 22), 1 requirement with a ReqACo between $25 \%$ and $30 \%$ (e.g., 6 axioms out of 22), 1 requirement with a ReqACo between $35 \%$ and $40 \%$ (e.g., 8 axioms out of 22 ) and 1 requirement with more than $50 \%$ (e.g., 11 axioms out of 22).

To analyse the influence of the complexity of requirements regarding other aspects of the development process, it was compared with the number of defined tests (NTests), the development time (VDevTime) and the size of the ontologies during the sprints $(N A)$.

Influence of the complexity of requirements on the number of tests. First, the complexity of the requirements was compared with the number of tests (NTests) defined for each ontology in order to determine whether the complexity of requirements influences the number of defined tests. However, from the information depicted in Figure 7.13 and in Table 7.12 it can be deduced that the complexity of requirements does not influence the number of tests, since the most complex requirements are associated with the Core ontology, but no additional tests were defined. Additionally, the Datatypes ontology, which has simple requirements, is the ontology with most additional tests.

The correlation coefficient between the average complexity of requirements (Re$q A C o$ ) and the average tests defined during the development process for each ontology (NTests) is -0.5. From this correlation coefficient in can be concluded that although there might be an inverse relationship between the complexity of requirements and the number of tests, it is not significant enough to be considered in this use case. 


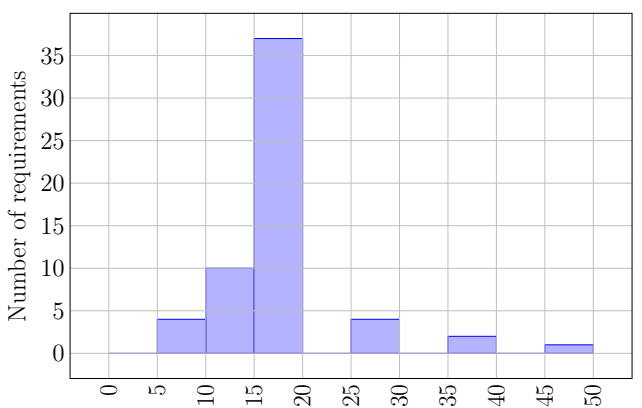

Requirement Axiom Complexity (ReqACo)

(a) Core ontology

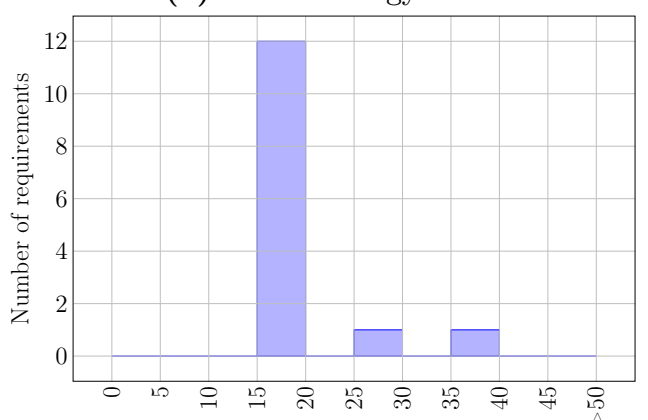

Requirement Axiom Complexity (ReqACo)

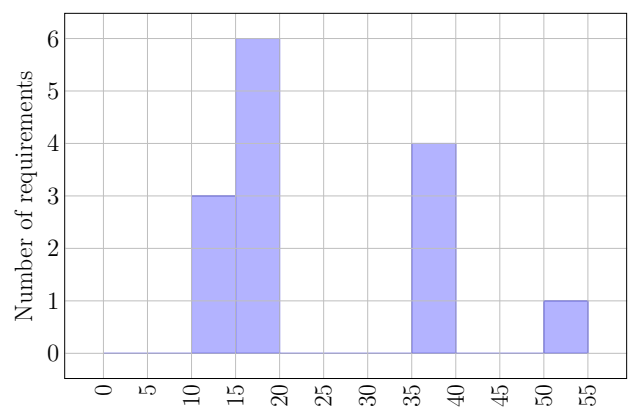

Requirement Axiom Complexity (ReqACo)

(b) WoT ontology

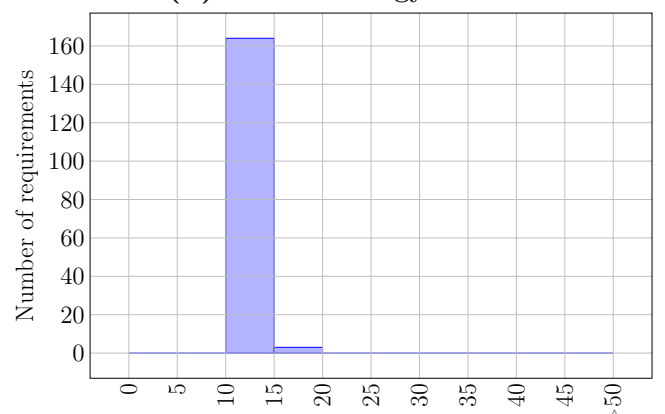

Requirement Axiom Complexity (ReqACo)

(c) Mappings ontology

(d) Adapters ontology

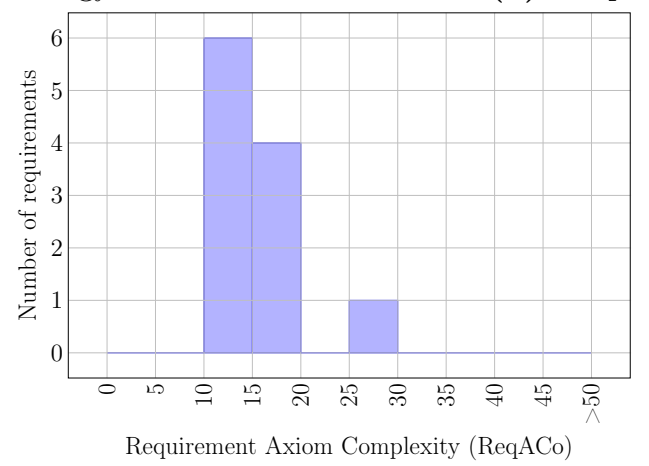

(e) Datatypes ontology

Figure 7.13: Distribution of the requirements axiom complexity in each ontology in the last sprint

Influence of the complexity of requirements on the ontology development time. The complexity of requirements was also compared with the time spent in the development process. Figure 7.13 illustrates that the ontology with the most complex requirements is the WoT ontology while the ontology with the highest required development time (Figure 7.12) was the Core ontology. 
The correlation coefficient between the average complexity of requirements and the average time spent per requirement is -0.7 . Therefore, the complexity of requirements influences negatively the development time of ontologies, i.e., the higher the requirement complexity, the lower the development time per requirement. These results can be derived from the fact that the WoT ontology, whose requirements have an average of 14.57 ReqACo, needs an average time of 10.79 days to develop each requirement, while Core, whose requirements have an average of 21.2 ReqACo needs 0.57 days to develop each requirement. The data used to calculate these metrics is taken from the GitHub repository where the commits related to these ontologies are stored. These results sound counter-intuitive, establishing that the higher the complexity, the lower the development time. Therefore, intending to analyse whether other factors affect this relationship, the complexity of requirements results were joined with the number of requirements to determine whether its combination affects the development time.

If this complexity of requirements result is joined with Table 7.12 , it can be observed that the number of defined requirements in the Core ontology outnumbered the defined requirements of almost the rest of ontologies. Moreover, the Core ontology has also requirements with significant complexity. In order to check whether the complexity together with the number of requirements influences the development time of the ontology, the coefficient of multiple correlation was calculated between the average requirements complexity (ReqACo), the total number of requirements (NReqs) and the average development time (VDevTime) for the last sprint of the ontologies. This correlation coefficient had a value of 0.82 . Therefore, it can be concluded that the joint information obtained by the complexity and the number of requirements influences the development time of ontologies.

Influence of the complexity of requirements on the ontology size. Finally, the complexity of requirements was also compared with the size of the ontologies $(N A)$, which are shown in Figure 7.9. This figure shows that the sizes of both the Core ontology and the Adapters ontology exceed the size of the other ontologies. However, from Figure 7.13 it can be noticed that the WoT ontology is the ontology with most complex requirements.

The correlation coefficient between the average complexity of requirements (Re$q A C o$ ) and the average size of each ontology $(N A)$ is -0.4 . Therefore, with all this information it can be concluded that the complexity of requirements does not influence the size of ontologies. 


\subsubsection{Influences derived from the volatility of requirements}

Volatility of the Ontology Requirement Specification Document (ORSD) refers to the modifications of the requirements over time (ReqVolatility), which can be divided into additions (NAddedReq) or deletions (NRejectedReq) and total accepted requirements (NAcceptedReq). Figure 7.14 presents the number of additions of requirements (NAdde$d R e q$ ), the number of rejections of requirements (NRejectedReq) and the number of acceptances of requirements (NAcceptedReq) for the five ontologies during their development iterations.

Figure 7.14 illustrates that in the WoT and Mappings ontologies the majority of the requirements were identified at the beginning of the development process, even though several of them were rejected during the development. Nevertheless, in the Core ontology the requirements changed over time, adding or deleting at least one requirement in each iteration. In the case of the Adapters and the Datatypes ontologies no additional information could be extracted since they were created in a single sprint.

This ReqVolatility was compared with the ontology volatility (OVolatility). Table 7.14 shows the number of changed axioms (added and removed) in each sprint. If the values that are shown in Figure 7.14 are compared with the ones shown in Table 7.14 it can be observed that the ontology with more volatility in their requirements is the Core ontology, which is also the ontology with more changes according to the added and removed axioms over time. Table 7.14 shows that the Core ontology has at least 10 axioms changed in each of the six sprints.

Table 7.14: Volatility of the ontologies (OVolatility) during each sprint

\begin{tabular}{|l|r|r|r|r|r|r|}
\cline { 2 - 7 } \multicolumn{1}{c|}{} & Sprint 1 & Sprint 2 & Sprint 3 & Sprint 4 & Sprint 5 & Sprint 6 \\
\hline Core & 105 & 40 & 374 & 234 & 215 & 10 \\
\hline WoT & 151 & 6 & 1 & 8 & - & - \\
\hline Mappings & 59 & 28 & - & - & - & - \\
\hline Adapters & - & - & - & - & - & 1226 \\
\hline Datatypes & - & - & - & - & - & 94 \\
\hline
\end{tabular}

Moreover, the correlation coefficient between the changes in the requirements (Re$q$ Volatility) and in the ontologies (OVolatility) is 0.64 . Figure 7.15 depicts the related scatterplot, which shows the grouping of values from 0 to 50 changed requirements. Therefore, it can be concluded that the volatility of requirements influences the volatility of ontologies. 


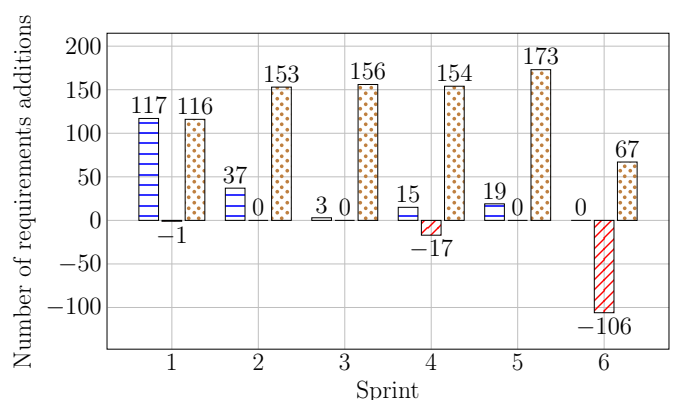

\begin{tabular}{cc}
\hline$V_{\square}$ & Added requirements \\
Rejected requirements
\end{tabular}

Total accepted requirements

(a) Core ontology

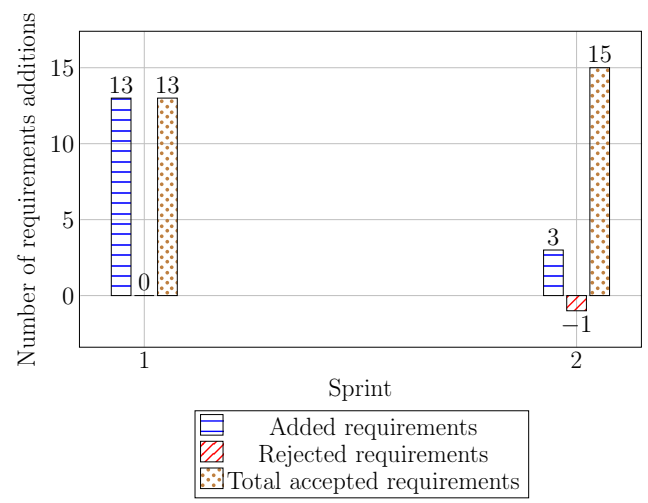

(c) Mappings ontology



Added requirements
Rejected requirements

Total aled requiremients

(b) WoT ontology

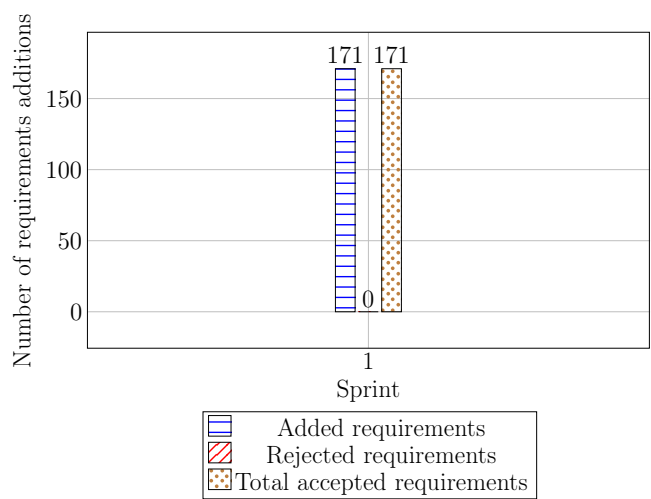

(d) Adapters ontology

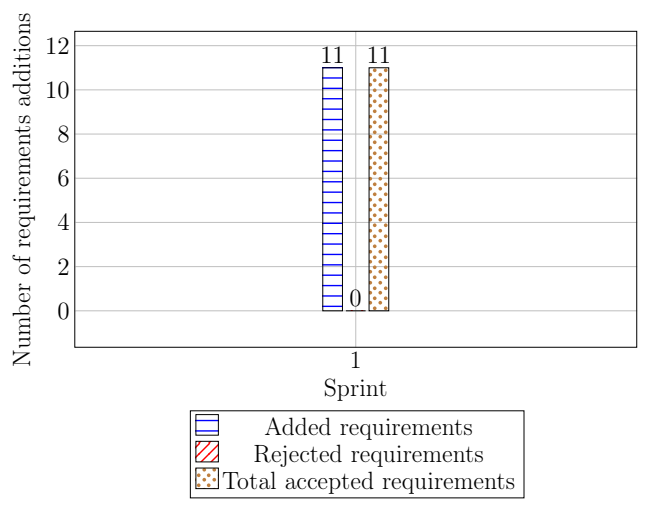

(e) Datatypes ontology

Figure 7.14: Distribution of the volatility of the requirements in each ontology in the last sprint 


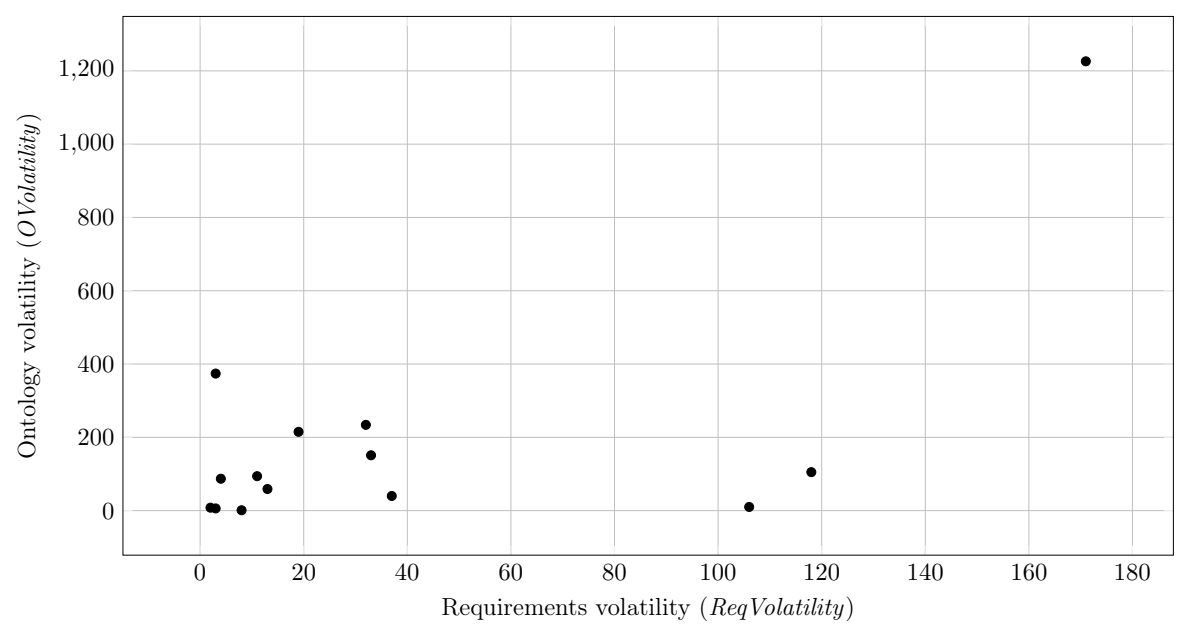

Figure 7.15: Scatterplot related to the requirements volatility (ReqVolatility) and the ontology volatility (OVolatility)

\subsubsection{Influences derived from the number of tests}

Team leaders and ontology engineers need to analyse the ontology coverage, in order to be aware of the requirements planned for each iteration and to determine whether they are satisfied by the ontology or if the generated tests cover all the requirements. Stakeholders also make use of this information in order to be aware of whether the ontologies satisfy their needs. For the five ontologies sets of tests have been defined in order to verify whether the ontological requirements are satisfied by the analysed ontologies. Table 7.12 summarises the number of defined tests (NTests) per sprint. As it can be observed, the defined tests for the Core and the Adapters ontologies outnumber the tests defined for the rest of the ontologies.

To analyse the effect of the number of defined tests in the development process, it has been compared with the number of tested terms (TestEffRatio). Figure 7.16 shows the percentage of tested terms (TestEffRatio) during the sprints for each analysed ontology. Only the Adapters ontology has $100 \%$ of its terms tested, while the values in the Core and the WoT ontologies do not exceed 53\%. These results are normal due to the fact that there are terms that are not defined in the requirements. These terms can be created from the addition of ontology design patterns (Gangemi and Presutti, 2009), the creation of hierarchies, the creation of n-ary relations (Noy et al., 2006), or the reuse of terms from other ontologies. However, a high value of TestEffRatio shows that the ontology is generated almost directly from the requirements, without many modelling decisions of the ontology engineers. In the case of Mappings, Figure 7.16 shows that 
the TestEffRatio values are extremely low. After analysing the cause of these results, it was deduced that the Mappings ontology imports the WoT and Core ontologies but their terms are not tested in the Mappings test suite because they are not identified in the requirements.



Sprint

Tested Term Percentage (TestEffRatio) Covered Requirement Percentage (CovReqPt) Tested Requirement Percentage (TestedReqPtc)

(a) Core ontology

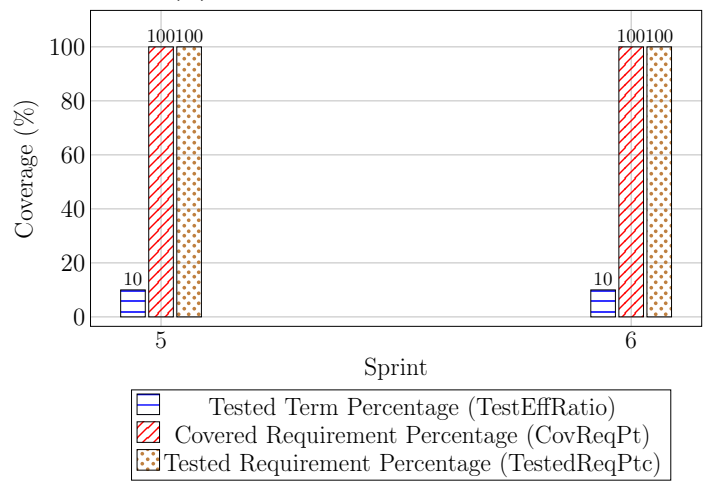

(c) Mappings ontology

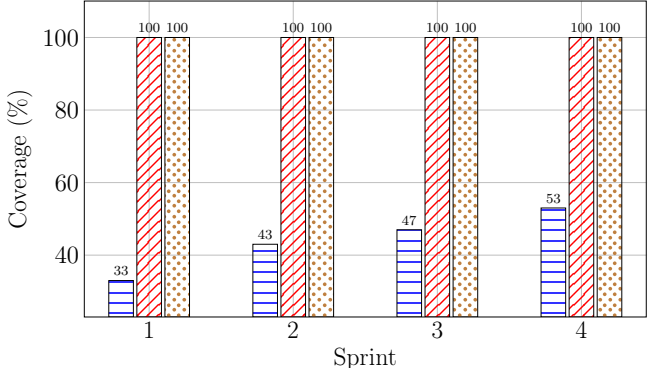

Tested Term Percentage (TestEffRatio) $\triangle$ Covered Requirement Percentage (CovReqPt) Tested Requirement Percentage (TestedReqPtc)

(b) WoT ontology

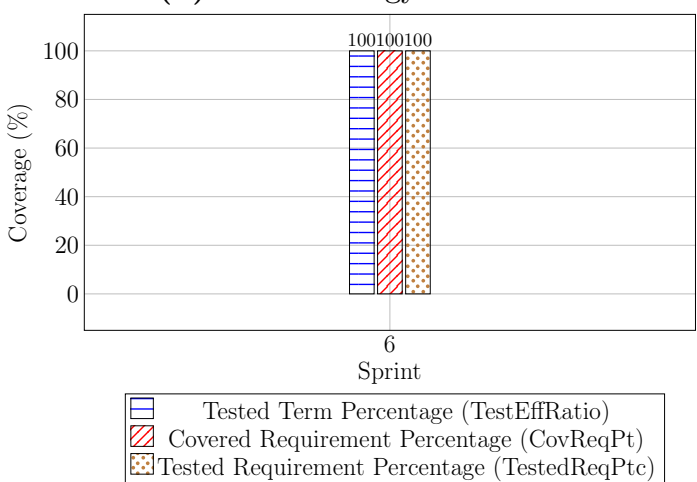

(d) Adapters ontology

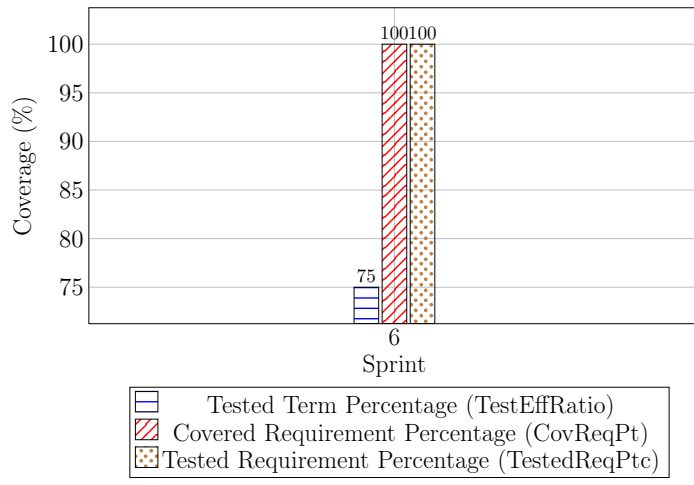

(e) Datatypes ontology

Figure 7.16: Distribution of the test and requirement coverage in each ontology version

As mentioned before, the ontologies with the highest number of tests are the Core 
and the Adapters ontologies, with 173 defined tests in sprint 5 and 171 defined tests in sprint 6, respectively. However, their TestEffRatio metric differs significantly, being $49 \%$ for the Core ontology and $100 \%$ for the Adapters ontology. Therefore, with all these results and a correlation coefficient of 0.36 , it can be concluded that the number of tests does not influence the number of tested terms in ontologies.

\subsubsection{Influences derived from the number of tested terms}

Finally, the number of tested terms (TestEffRatio) was also compared with the coverage of the ontology (CovReqPtc). Figure 7.16 depicts the CovReqPtc for the ontologies in each sprint. It can be observed that the Core and the WoT ontologies, even though the tested terms do not exceed $53 \%$, reach $100 \%$ of the coverage of the ontology. Similarly, the Adapters ontology has 100\% of its terms tested and also reaches $100 \%$ of the coverage of the ontology. This could occur because, due to modelling decisions or ODPs, new terms were added to the ontology but avoided in the requirements. Moreover, the correlation coefficient between these two metrics is 0.002 . Therefore, the number of tested terms does not influence the coverage of requirements.

\subsubsection{Conclusions of the experiment}

With respect to the hypothesis and based on the information retrieved in this experiment, the following conclusions can be outlined:

- H2. There is a direct correlation between some metrics related to functional requirements and those metrics related to the implementation and the tests of the ontology.

The metrics presented in Chapter 5 were applied in a real use case, providing information in order to monitor the development process. The experiment was used to compare how some of the metrics influence others. Although it was found that some of the analysed metrics did not cause any effect in the implementation and the tests, the metrics calculated in the use case provided insights about the development process of the analysed ontologies, and confirm some effects between the requirements, the implementation and the tests. Figure 7.17 shows the results of the experiments, identifying which of the analysed metrics influence others. As an example, it was found that the number of requirements and the complexity of requirements jointly influence the development time. Therefore, there is a direct correlation between such metrics related to the requirements and the implementation of an ontology. Moreover, since in this use case the tests were extracted 
from the requirements, the extension of the requirements influences the extension of the tests. Consequently, the hypothesis is true.

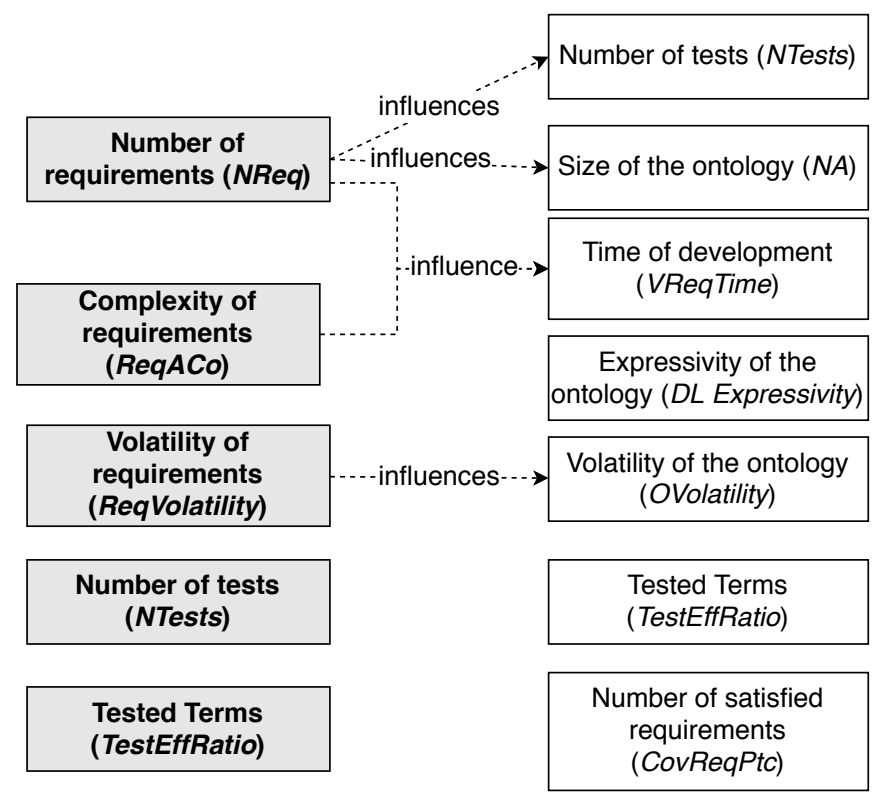

Figure 7.17: Influences between metrics obtained during the experiment

\subsection{Evaluation of the method for ontology conformance testing}

Chapter 6 presented the method for ontology conformance testing based on ontological requirements. This method identifies the requirements that are shared among an ontology and a standard, as well as the requirements that lead to a conflict between them. The aim of this method is to identify whether the ontology is conformant with such standards. This section describes the experiments performed in order to determine the type of requirements that are shared between an ontology and a set of standards related to the same domain. The following hypothesis is made for this evaluation (Section 3.3):

- H3. The majority of functional requirements shared by a set of ontologies from the same domain are related to the definition of terms and to the existence of relations between classes, while more restrictive requirements such as cardinalities are not frequently shared. 
To validate the hypothesis, two experiments were performed: (1) following the conformance testing method described in Section 6.1, it was analysed the conformance between an ontology and a set of standards related to the same domain; and (2) following the minimum common knowledge identification method proposed in Section 6.2, the minimum common knowledge between the aforementioned standards was analysed. The first experiment provided insights about the hypothesis, while the latter one confirmed it.

\subsubsection{Conformance testing method}

The first experiment was oriented to the analysis of the conformance of an ontology with regards to a set of standards related to the same domain.

\subsubsection{Experiment design}

The goal of this experiment was to determine which type of requirements related to standards were satisfied by a given ontology. To that end, an ontology network already published on the Web was collected, together with a set of standards related to the same domain.

These standards need to have associated requirements that identify the knowledge defined in them or documentation that allows extracting the requirements. Afterwards, the tests associated with these requirements were defined following the testing process and testing language described in Chapter 4. Moreover, as explained in Chapter 6, these tests were abstract and did not have any information about ontologies such as URIs, making them independent of the ontology from which they are extracted. Therefore, these tests can be executed on any ontology even though they were extracted from standards.

To analyse the type of shared requirements, the conformance testing method described in Section 6.1 was applied, where tests associated with the requirements of each standard are executed on each ontology. Those requirements that are satisfied by the ontology are considered as shared requirements.

The following steps summarise the actions performed during the experiment:

1. To gather requirements related to the standards.

2. To group them according to their related topic, as described in Section 6.1.1.1.

3. To generate the abstract tests associated with these requirements, following the testing process and testing language described in Chapter 4. 
4. To group the tests according to the type of test and associated topic.

5. To execute the tests on the ontology network.

6. To analyse the results in order to identify classes and properties that are shared between each standard and the ontology.

The following information was retrieved from the performance of these steps for each ontology in the ontology network:

- The types of the tests associated with the requirements of the standards that are passed by the ontology.

- The topics related to the tests associated with the requirements of the standards that are passed by the ontology.

- The results of the tests associated with the requirements of the standards that were executed on the ontology.

- The classes and properties that are shared between each standard and the ontology to be analysed based on the results of the tests.

In order to support the execution of tests, the Themis tool, which is described in Section 4.4, was used. Themis executed all the tests associated with the standards on each ontology and identified which were the tests that were passed by the ontology and which were not. Afterwards, a manual analysis to identify the shared types, topics and terms was performed.

\subsubsection{Results and discussion}

The ontology network that was developed in the European VICINITY project was selected for this experiment. This ontology network, as mentioned in Section 7.1, includes five ontologies, i.e., the VICINITY Core (Core), the Web of Things (WoT), the WoT mappings (Mappings), the VICINITY Adapters (Adapters), and the Datatypes (Datatypes) ontologies. The Core ontology represents the information exchanged about IoT descriptor data between peers through the VICINITY platform; this ontology is being created by following a cross-domain approach and implements requirements from different domain experts. The WoT ontology aims to model the Web of Things domain according to the W3C WoT Working Groun ${ }^{53}$ descriptions. The Mappings ontology represents the mechanism for accessing the values provided by web things in the VICINITY

\footnotetext{
$5 \sqrt[3]{\text { https: / / www.w3.org/WoT/WG/ }}$
} 
platform. The Adapters ontology aims to model all the different types of devices and properties that can be defined in the VICINITY platform. Finally, the Datatypes ontology aims to model the required and provided datatypes that are used in the interaction patterns of the platform. These five ontologies are of different sizes, had different restrictions defined and, although they are related to the same field, they are focused on different aspects of it.

The VICINITY network is related to the IoT field. Therefore, the following IoT standards were selected for the experiment: (1) the ETSI SAREF ontology, which describes smart applications; (2) the W3C SSN ontology, which describes sensors, their observations, and related procedures; and (3) the oneM2M base ontology, which provides syntactic and semantic interoperability of oneM2M platforms with external systems. Moreover, the conformance with the following standard documents in the IoT field was analysed: (1) the ISO/IEC 30141:2017 ISO (2017), which describes the IoT Reference Architecture for connecting systems; and (2) the OCF standard 54 which describes devices and how they interact. It should be mentioned that neither the OCF standard nor the ISO/IEC 30141 have related ontologies. The set of requirements and tests associated with these standards is available online 55

The requirements related to the aforementioned standards were collected as both competency questions and natural language statements. Table 7.15 summarises the list of standards, the provenance of the gathered requirements associated with such standards, the number of ontology requirements and the number of defined tests. As it can be observed, some of the requirements were extracted from official documentation, e.g., Technical Reports, while others were extracted from online specifications. Additionally, in this experiment it was defined one test per requirement.

The W3C SSN, the OCF and the ISO/IEC 30131 do not have requirements defined in their official documentation and specifications. Therefore, based on the documentation associated with the standards, a set of requirements were extracted for each one. The CORAL corpus, which is described in Section 4.1 and includes examples of requirements and their patterns, was used as a guideline to define them. These requirements identified the classes, properties and relations between classes that are defined in each standard. For the W3C SSN standard, the requirements are mainly related to observation and actuation, therefore, not all the classes and properties defined in the standard are included in the list of requirements.

From the results obtained by following this ontology conformance testing method,

$5^{54}$ https://openconnectivity.org

55 http://vicinity.iot.linkeddata.es/vicinity/conformance.html 
Table 7.15: Summary of requirements information for the IoT standards

\begin{tabular}{|c|c|c|c|c|}
\hline Ontology & Version & Requirements provenance & Requirements & Tests \\
\hline $\begin{array}{l}\text { ISO/IEC } \\
30141\end{array}$ & - & $\begin{array}{l}\text { ISO/IEC 30141:Internet of Things } \\
\text { (IoT) - Reference Architecture (ISO, } \\
\text { 2017) }\end{array}$ & 35 & 35 \\
\hline $\mathrm{OCF}$ & $\mathrm{v} 2.0 .2$ & OCF Core Specification (OCF, 2019) & 27 & 27 \\
\hline oneM2M & v3.6.0 & $\begin{array}{l}\text { SAREF extension investigation Tech- } \\
\text { nical Report (TR } 103 \text { 411) (ETSI, } \\
2020 \text { ) }\end{array}$ & 33 & 33 \\
\hline SAREF & $\mathrm{v} 2.1 .1$ & $\begin{array}{l}\text { SAREF extension investigation Tech- } \\
\text { nical Report (TR } 103 \text { 411) (ETSI, } \\
2020 \text { ) }\end{array}$ & 68 & 68 \\
\hline $\mathrm{SSN}$ & $\mathrm{V} 2.0$ & $\begin{array}{l}\text { W3C SSN Specification Haller et al. } \\
\text { 2017) }\end{array}$ & 34 & 34 \\
\hline
\end{tabular}

which are summarised in Table 7.16, it can be deduced that the VICINITY ontology network passed 42 tests related to the standards, but did not take into consideration some concepts related to them as it is shown in the 138 requirements with undefined terms result. However, it can also be concluded that there are no conflicts between the VICINITY ontology network and these standards since there are no tests with the conflict result, even though there are some absences, i.e., the terms specified in the standard are defined in the VICINITY ontology network but the relations between the terms are not. Therefore, it can be concluded that there are no inconsistencies between the domain defined in the standards and the domain defined in the VICINITY ontology network.

Table 7.16: Summary of testing results of the IoT standards for the VICINITY ontology network

\begin{tabular}{|c|c|c|c|c|}
\hline \multirow{2}{*}{ Standard } & \multicolumn{4}{|c|}{ Test results } \\
\hline & Passed & Undefined term & Absent & Conflict \\
\hline ISO/IEC 30141 & $12(34.26 \%)$ & $19(54.28 \%)$ & $4(11.42 \%)$ & 0 \\
\hline OCF & $9(33.33 \%)$ & $17(62.93 \%)$ & $1(3.70 \%)$ & 0 \\
\hline oneM2M & $2(6.06 \%)$ & $30(90.90 \%)$ & $1(3.03 \%)$ & 0 \\
\hline SAREF & $7(10.29 \%)$ & $61(89.70 \%)$ & $0(0 \%)$ & 0 \\
\hline SSN & $12(35.29 \%)$ & $11(32.35 \%)$ & $11(32.35 \%)$ & 0 \\
\hline Total & $42(21.32 \%)$ & $138(70.00 \%)$ & $17(8.63 \%)$ & 0 \\
\hline
\end{tabular}

Table 7.16 also shows in parentheses the Passed Test Percentage, Undefined Test Percentage and Absent Test Percentage metrics for each standard, from which it can 
be observed that the majority of the tests result in undefined term. Those undefined terms in the tests identify the terms that are out scope of the VICINITY domain.

As an example, the SAREF ontology has $89.7 \%$ of Undefined Test Percentage, which reflects that $89.7 \%$ of the requirements defined in the SAREF specification have terms that are not included in the domain described by VICINITY. Therefore, from this table it can be concluded that the overlap between the VICINITY ontology network and SAREF is minimum. The same happens with the oneM2M ontology, which has $90.9 \%$ of Undefined Test Percentage.

In the case of the ISO/IEC 30141, the OCF and the W3C SSN the Passed Test Percentage is higher than $30 \%$, indicating that there are overlaps between them and the VICINITY ontology network. It should be considered that the VICINITY ontology network imports the SOSA ontology, which is a module included in the SSN ontology and, therefore, several terms defined in the SSN ontology are already defined in the VICINITY ontology.

Additionally, Table 7.17 was created with the aim of analysing the types of tests passed by the VICINITY ontology network. As shown in this table, requirements are only divided into four types, i.e., definition of classes, subsumption, relation between terms and cardinality. Other restrictions, e.g., functional or symmetric properties, were not found in this set of requirements. Table 7.17 shows the Passed Test Percentage grouped according to the type of test, e.g., in the case of the SAREF ontology, the VICINITY ontology network passed $40 \%$ of tests related to the definition of classes. From this table it can be observed that the type of test with the highest Passed Test Percentage is the definition of classes, although there is a small number of this type of tests defined in the test suite. This occurs due to the fact that in the requirements specifications there are a small number of requirements related to only the definition of classes. Furthermore, the tests related to cardinalities were not passed by the VICINITY ontology.

Table 7.17: Summary of the types of tests passed by the VICINITY ontology

\begin{tabular}{|c|c|c|c|c|c|c|c|c|c|c|}
\hline & \multicolumn{2}{|c|}{ ISO 30141} & \multicolumn{2}{|c|}{$\mathrm{OCF}$} & \multicolumn{2}{|c|}{ oneM2M } & \multicolumn{2}{|c|}{ SAREF } & \multicolumn{2}{|c|}{ SSN } \\
\hline Type of test & Total & Passed & Total & Passed & Total & Passed & Total & Passed & Total & Passed \\
\hline Definition of classes & 0 & - & 1 & $100 \%$ & 6 & $16.67 \%$ & 7 & $40 \%$ & 8 & $87.5 \%$ \\
\hline $\begin{array}{l}\text { Subsumption between } \\
\text { classes }\end{array}$ & 7 & $85.71 \%$ & 4 & $0 \%$ & 4 & $0 \%$ & 17 & $17.64 \%$ & 3 & $66.67 \%$ \\
\hline $\begin{array}{l}\text { Terms relation } \\
\text { (universal and existential } \\
\text { restrictions) }\end{array}$ & 23 & $26.08 \%$ & 21 & $38.09 \%$ & 20 & $5 \%$ & 40 & $5 \%$ & 20 & $15 \%$ \\
\hline Cardinalities & 5 & $0 \%$ & 1 & $0 \%$ & 3 & $0 \%$ & 6 & $0 \%$ & 3 & $0 \%$ \\
\hline
\end{tabular}


Table 7.18 shows the topics related to the requirement 56 of the IoT standards and the percentage of tests passed by the VICINITY ontology network associated with each one. In this use case, each topic refers to a class of the ontology that can be extracted from each requirement together with its properties.

Table 7.18: Summary of the topics of each standard and the Passed Test Percentage of the VICINITY ontology network

\begin{tabular}{|c|c|c|c|}
\hline Ontology & Topic & № requirements & Passed Test Percentage \\
\hline \multirow{7}{*}{ ISO/IEC 30141} & Thing & 9 & $60 \%$ \\
\hline & Accessibility & 9 & $21.43 \%$ \\
\hline & Device & 9 & $28.57 \%$ \\
\hline & Service & 8 & $25 \%$ \\
\hline & User & 4 & $100 \%$ \\
\hline & Data store & 2 & $0 \%$ \\
\hline & Actuator & 1 & $100 \%$ \\
\hline \multirow{10}{*}{$\mathrm{OCF}$} & Device & 14 & $35.71 \%$ \\
\hline & Resource & 15 & $0 \%$ \\
\hline & Endpoint & 3 & $33.33 \%$ \\
\hline & Thing & 2 & $0 \%$ \\
\hline & Aspect & 1 & $0 \%$ \\
\hline & Function & 1 & $0 \%$ \\
\hline & Link & 1 & $0 \%$ \\
\hline & Maintenance & 1 & $0 \%$ \\
\hline & Property & 1 & $0 \%$ \\
\hline & Security & 1 & $0 \%$ \\
\hline \multirow{12}{*}{ oneM2M } & Service & 7 & $0 \%$ \\
\hline & Function & 6 & $0 \%$ \\
\hline & Operation & 6 & $0 \%$ \\
\hline & Device & 5 & $100 \%$ \\
\hline & Accessibility & 4 & $0 \%$ \\
\hline & Thing & 3 & $33.33 \%$ \\
\hline & Aspect & 2 & $0 \%$ \\
\hline & Communication & 2 & $0 \%$ \\
\hline & Task & 2 & $0 \%$ \\
\hline & Metadata & 1 & $0 \%$ \\
\hline & Network & 1 & $0 \%$ \\
\hline & Profile & 1 & $0 \%$ \\
\hline \multirow{5}{*}{ SAREF } & Device & 46 & $10.86 \%$ \\
\hline & Function & 19 & $0 \%$ \\
\hline & Service & 6 & $0 \%$ \\
\hline & Property & 5 & $0 \%$ \\
\hline & Building & 4 & $25 \%$ \\
\hline \multirow{4}{*}{ SAREF } & Command & 3 & $0 \%$ \\
\hline & Commodity & 2 & $0 \%$ \\
\hline & Profile & 2 & $0 \%$ \\
\hline & Price & 1 & $0 \%$ \\
\hline
\end{tabular}

\footnotetext{
${ }^{56}$ Note that a requirement can belong to one or more topics.
} 
Table 7.18 - Continued from previous page

\begin{tabular}{|l|l|r|r|}
\hline Ontology & Topic & No requirements & Passed Test Percentage \\
\hline \multirow{4}{*}{ SSN } & Time & 1 & $0 \%$ \\
\hline \multirow{5}{*}{} & Property & 7 & $100 \%$ \\
\cline { 2 - 4 } & Stimulus & 6 & $0 \%$ \\
\cline { 2 - 4 } & System & 6 & $40 \%$ \\
\cline { 2 - 4 } & Feature of interest & 5 & $40 \%$ \\
\cline { 2 - 4 } & Procedure & 5 & $33.33 \%$ \\
\cline { 2 - 4 } & Sensor & 5 & $75 \%$ \\
\cline { 2 - 4 } & Observation & 4 & $20 \%$ \\
\cline { 2 - 4 } & Results & 4 & $0 \%$ \\
\cline { 2 - 4 } & Deployment & 3 & $50 \%$ \\
\cline { 2 - 4 } & Observable Property & 3 & $50 \%$ \\
\cline { 2 - 4 } & Actuator & 2 & $66.66 \%$ \\
\cline { 2 - 4 } & Sample & 2 & $0 \%$ \\
\hline
\end{tabular}

From Table 7.18 it can also be observed that the VICINITY ontology network passed at least one test of the majority of the SSN and ISO/IEC 30141 topics. However, in the case of the SAREF ontology, the VICINITY ontology network only passes $10.86 \%$ of the tests related to devices. For the rest of standards, it can be observed that the majority of topics has $0 \%$ of Passed Test Percentage, i.e., none of the tests related to those topics were passed by the VICINITY ontology. However, there are some topics with $100 \%$ of Passed Test Percentage, which indicates that the ontology network is compliant with the requirements from the standards associated with such topics.

Moreover, it can also be observed that the topics which had more passed tests are related to high-level terms, e.g., Device, Sensor, Actuator or Thing. These terms are used in the majority of scenarios in the IoT field, regardless of the area of concern to be described. However, those terms that are particular to a use case, e.g., Data store, Network or Metadata, were only included in those ontologies focused on that use case.

Finally, for illustrating the conformance between the VICINITY ontology network and the IoT standards, Figure 7.18 was created, where arrows with white triangles on the top represent a subsumption relation between two classes. The origin of the arrow is the class to be declared as subclass of the class at the destination of the arrow. In addition, directed arrows are used to represent object properties between classes and parentheses represent cardinality restrictions. These figures show with dotted lines those properties and classes that are shared between each of the standard ontologies and the VICINITY ontology network. It is worth noting that the conceptualisations in Figure 7.18 which represent the OCF and the ISO/IEC 30141 standards were generated from the functional requirements since there are no ontologies related to them.

After analysing all the results obtained by the conformance method, it can be concluded that there are several requirements shared between the analysed ontology and 


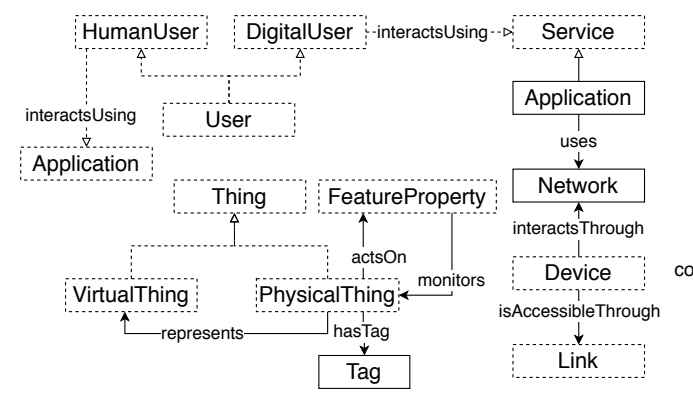

(a) ISO/IEC 30141 vs VICINITY

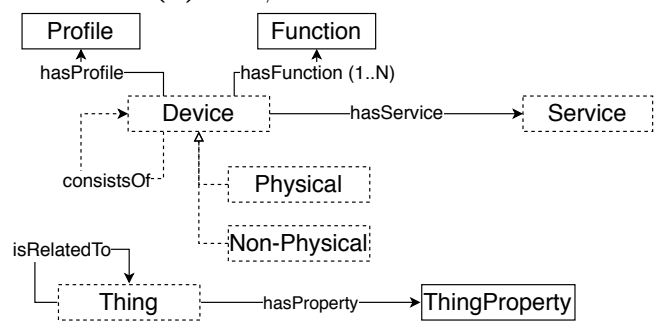

(c) oneM2M vs VICINITY

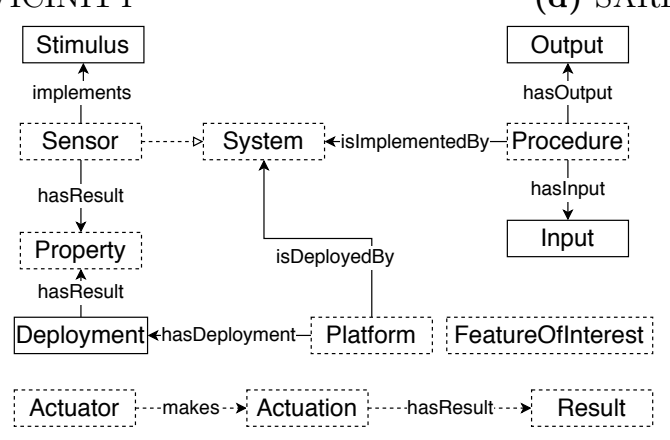

(e) SSN vs VICINITY

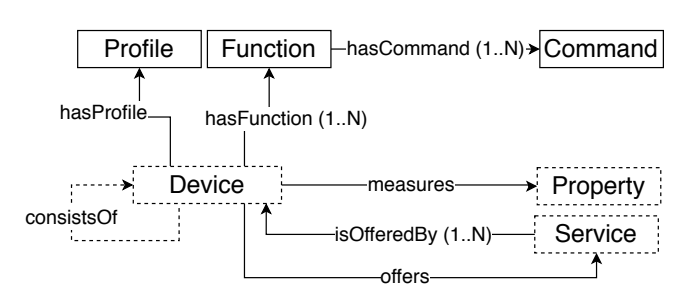

(d) SAREF vs VICINITY

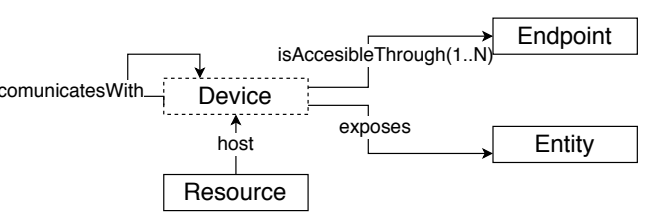

(b) OCF vs VICINITY

Procedure

hasinpu

Input

\section{(1)}

$$
\text { : }
$$

Figure 7.18: Shared properties and classes between IoT standards and the VICINITY ontology network

each standard. However, these shared requirements were mostly related to the definition of classes, as it is confirmed in Figure 7.18. The requirements that were more specific, such as those related to cardinalities, were only satisfied by the standard from which the requirements were extracted, although there can be some exceptions for example if an ontology imports a standard.

Not only those requirements related to the definition of tests are satisfied by the ontology, but also those related to relations between terms. However, the number of passed tests associated with these requirements is smaller. In this use case, ontologies and standards share those simple requirements that are related to classes or relations. This situation can stem because more complex restrictions are defined for particular 
cases when describing a field, and, therefore, they are not common to any scenario of such field. Bear in mind that these results depend on the quality of the specification of requirements: the more requirements, the more accurate the conformance analysis.

To conclude, the results provided by Tables 7.16 and 7.17 indicate that a set of standards and ontologies in the same domain can share a set of requirements; however, the number of these shared requirements might be small, and mostly related to the definition of classes. Moreover, this type of requirements is not common in requirements specification documents, as it can be observed in Table 7.17 .

Table 7.16 shows that only $21.32 \%$ of the tests associated with the requirements of the IoT standards are satisfied by the VICINITY ontology. Moreover, from Table 7.17 it can be observed that the tests related to the definition of classes were the tests with the highest Passed Tests Percentage. This information indicates that hypothesis H3 could be validated. In order to have more information to validate such hypothesis, the second experiment, i.e., the analysis of minimum common knowledge, was performed.

\subsubsection{Minimum common knowledge between ontologies}

In addition to experiment related to the conformance of an ontology with regards to a set of standards, it was also analysed the minimum common knowledge between such standards, following the method described in Section 6.2.

\subsubsection{Experiment design}

The aim of this experiment was to identify the common knowledge shared by a set of standards in order to check whether there is an overlap in the domain they describe. Therefore, the minimum common identification method described in Section 6.2 was applied. This experiment allowed to further analyse the type of shared requirements between standards.

As for the previous experiment, the standards needed to have associated requirements that identify the knowledge defined in them or documentation that allows extracting the requirements. However, they do not need to have an ontology associated. As it was performed for the previous experiment, the tests associated with these requirements were defined following the testing process and testing language described in Chapter 4. In this scenario, all the tests were also abstract, i.e., independent of the ontology from which they are extracted, and executed on all the standards.

The following steps summarise the actions performed during the experiment:

1. To gather requirements related to the standards. 
2. To group them according to the topic associated, as described in Section 6.1.1.1.

3. To generate the abstract tests associated with these requirements were defined following the testing process and testing language described in Chapter 4.

4. To group the tests according to the type of test and topic associated.

5. To execute the tests of all the standards on all the standards.

6. To analyse the results in order to identify classes and properties that are shared between more than one standard.

The following information was retrieved from the performance of the previous steps:

- The requirements that are shared between more than one standard.

- The types of the requirements shared between more than one standard.

- The topics related to the requirements shared between more than one standard.

- The classes and properties that are shared between more than one standard. These results allow to identify the layers of knowledge described in Section 6.2 for shared knowledge between models, namely, the common knowledge, which represents the knowledge shared by the set of analysed standards, and variant-domain knowledge, which represents the knowledge that is common to more than one standard.

To execute the tests, the extension of the tool Themis that automatically generates ontologies from their associated ATS for those standards without a related ontology was used. This automatic generation of ontologies allows the execution of the tests on any standard.

\subsubsection{Results and discussion}

For this experiment, the IoT standards used in the previous experiment, i.e., the ETSI SAREF ontology, the W3C SSN ontology, the oneM2M base ontology, the ISO/IEC 30141 and the OCF standard, were selected to be analysed. As mentioned in the previous section, neither the OCF standard nor the ISO/IEC 30141 have related ontologies.

After executing all the tests on all the IoT standards, the test results were analysed to check which requirements, and from which type and topic, were shared among the standards.

This analysis showed that none of the requirements and no terms were shared by all of the standards, i.e., the common knowledge. However, it could be identified the 
variant-domain knowledge, which refers to the knowledge shared by more than one standard. The results of such analysis are summarised in Table 7.19, where the ontologies that satisfy each requirement are indicated, as well as the type and topic associated with it. This table only includes those requirements that are shared by more than one ontology.

Table 7.19: Shared requirements between the IoT standards

\begin{tabular}{|c|c|c|c|c|}
\hline Provenance & Requirement & $\begin{array}{l}\text { Type of } \\
\text { requirement }\end{array}$ & Topic & $\begin{array}{l}\text { Standards that } \\
\text { satisfy the requirement }\end{array}$ \\
\hline $\begin{array}{l}\text { ISO/IEC } \\
30141\end{array}$ & $\begin{array}{l}\text { Actuators and sen- } \\
\text { sors are kinds of } \\
\text { IoT device }\end{array}$ & $\begin{array}{l}\text { Subsumption } \\
\text { between classes }\end{array}$ & Device & $\begin{array}{l}\text { ISO/IEC } 30141, \\
\text { SAREF }\end{array}$ \\
\hline $\mathrm{OCF}$ & $\begin{array}{l}\text { A device is a logical } \\
\text { entity }\end{array}$ & $\begin{array}{l}\text { Definition of a } \\
\text { class }\end{array}$ & Device & $\begin{array}{lr}\text { ISO/IEC } & 30141, \\
\text { OCF, } & \text { oneM2M, } \\
\text { SAREF } & \end{array}$ \\
\hline $\mathrm{OCF}$ & $\begin{array}{l}\text { A device can be } \\
\text { composed by other } \\
\text { devices }\end{array}$ & Terms relation & Device & $\begin{array}{ll}\text { OCF, } & \text { oneM2M, } \\
\text { SAREF } & \end{array}$ \\
\hline $\mathrm{OCF}$ & $\begin{array}{l}\text { A resource is a } \\
\text { physical thing in } \\
\text { the world }\end{array}$ & $\begin{array}{l}\text { Definition of a } \\
\text { class }\end{array}$ & Thing & $\mathrm{OCF}$, oneM2M \\
\hline oneM2M & $\begin{array}{l}\text { A controlling func- } \\
\text { tionality represents } \\
\text { a functionality that } \\
\text { has impacts on the } \\
\text { real world, but } \\
\text { does not gather } \\
\text { data }\end{array}$ & $\begin{array}{l}\text { Subsumption } \\
\text { between classes }\end{array}$ & Function & oneM2M, SAREF \\
\hline oneM2M & $\begin{array}{l}\text { A measuring func- } \\
\text { tionality represents } \\
\text { a functionality that } \\
\text { has no impacts on } \\
\text { the real world, but } \\
\text { only gathers data }\end{array}$ & $\begin{array}{l}\text { Subsumption } \\
\text { between classes }\end{array}$ & Function & oneM2M, SAREF \\
\hline oneM2M & $\begin{array}{l}\text { A device can be } \\
\text { composed of sev- } \\
\text { eral (sub-)devices }\end{array}$ & Terms relation & Device & oneM2M, SAREF \\
\hline oneM2M & $\begin{array}{l}\text { A thing is an entity } \\
\text { that can be identi- } \\
\text { fied in the oneM } 2 \mathrm{M} \\
\text { System. }\end{array}$ & $\begin{array}{l}\text { Definition of a } \\
\text { class }\end{array}$ & Thing & $\begin{array}{l}\text { ISO/IEC } 30141, \\
\text { oneM2M }\end{array}$ \\
\hline SAREF & $\begin{array}{l}\text { A device shall have } \\
\text { a model property }\end{array}$ & Terms relation & $\begin{array}{l}\text { Device, Prop- } \\
\text { erty }\end{array}$ & $\begin{array}{l}\text { ISO/IEC } 30141, \\
\text { OCF, } \\
\text { SAREF }\end{array}$ \\
\hline SAREF & What is a device? & $\begin{array}{l}\text { Definition of a } \\
\text { class }\end{array}$ & Device & $\begin{array}{lr}\text { ISO/IEC } & 30141, \\
\text { OCF, } & \text { oneM2M, } \\
\text { SAREF } & \end{array}$ \\
\hline
\end{tabular}


Table 7.19 - Continued from previous page

\begin{tabular}{|c|c|c|c|c|}
\hline Provenance & Requirement & $\begin{array}{l}\text { Type of } \\
\text { requirement }\end{array}$ & Topic & $\begin{array}{l}\text { Standards that } \\
\text { satisfy the requirement }\end{array}$ \\
\hline SAREF & $\begin{array}{l}\text { A device can op- } \\
\text { tionally have a de- } \\
\text { scription }\end{array}$ & Terms relation & Device & $\begin{array}{l}\text { ISO/IEC } 30141, \\
\text { OCF, } \\
\text { SAREF }\end{array}$ \\
\hline SAREF & $\begin{array}{l}\text { A device may con- } \\
\text { sist of other devices }\end{array}$ & Terms relation & Device & $\begin{array}{l}\text { ISO/IEC } 30141, \\
\text { oneM2M, SAREF }\end{array}$ \\
\hline SAREF & $\begin{array}{l}\text { A function rep- } \\
\text { resents the func- } \\
\text { tionality necessary } \\
\text { to accomplish the } \\
\text { task for which a } \\
\text { device is designed }\end{array}$ & Terms relation & $\begin{array}{l}\text { Device, Func- } \\
\text { tion }\end{array}$ & oneM2M, SAREF \\
\hline SAREF & $\begin{array}{l}\text { A service is a } \\
\text { representation } \\
\text { of a function to } \\
\text { a network that } \\
\text { makes this func- } \\
\text { tion discoverable; } \\
\text { registerable and re- } \\
\text { motely controllable } \\
\text { by other devices in } \\
\text { the network }\end{array}$ & Terms relation & $\begin{array}{l}\text { Service, Func- } \\
\text { tion, Device }\end{array}$ & oneM2M, SAREF \\
\hline SAREF & $\begin{array}{l}\text { A device can be } \\
\text { used for the pur- } \\
\text { pose of sensing }\end{array}$ & Terms relation & $\begin{array}{l}\text { Device, Func- } \\
\text { tion }\end{array}$ & oneM2M, SAREF \\
\hline SAREF & $\begin{array}{l}\text { A device can be } \\
\text { used for measuring } \\
\text { a property }\end{array}$ & Terms relation & $\begin{array}{l}\text { Device, Func- } \\
\text { tion }\end{array}$ & oneM2M, SAREF \\
\hline SAREF & $\begin{array}{l}\text { A command can } \\
\text { act upon a state } \\
\text { to represent that } \\
\text { the consequence of } \\
\text { a command can be } \\
\text { a change of state of } \\
\text { the device }\end{array}$ & $\begin{array}{l}\text { Definition of a } \\
\text { class }\end{array}$ & Command & oneM2M, SAREF \\
\hline SAREF & $\begin{array}{l}\text { A device offers a } \\
\text { service }\end{array}$ & Terms relation & Device, Service & oneM2M, SAREF \\
\hline SSN & What is a sensor? & $\begin{array}{l}\text { Definition of a } \\
\text { class }\end{array}$ & Sensor & $\begin{array}{l}\text { ISO/IEC 30141, } \\
\text { SAREF, SSN }\end{array}$ \\
\hline $\mathrm{SSN}$ & $\begin{array}{l}\text { What is an actua- } \\
\text { tor? }\end{array}$ & $\begin{array}{l}\text { Definition of a } \\
\text { class }\end{array}$ & Actuator & $\begin{array}{l}\text { ISO/IEC 30141, } \\
\text { SAREF, SSN }\end{array}$ \\
\hline $\mathrm{SSN}$ & $\begin{array}{l}\text { What is a prop- } \\
\text { erty? }\end{array}$ & $\begin{array}{l}\text { Definition of a } \\
\text { class }\end{array}$ & Property & $\begin{array}{l}\text { OCF, SAREF, } \\
\text { SSN }\end{array}$ \\
\hline
\end{tabular}

Table 7.19 shows that only 21 requirements out of 197 , i.e., $10.66 \%$ of the requirements, were shared between the analysed standards. This fact confirms that only a small number of requirements are shared between standards and ontologies related to the same domain. 
Moreover, from Table 7.19 it can be observed that the majority of these requirements were related to the definition of classes (e.g., the requirement "What is a sensor?" only checks that there is a Sensor in the ontology) and relationships between them (e.g., the requirement "A device offers a service" checks the relation between the classes Device and Service). This table also shows that only 8 topics had shared requirements, but the requirements related to the rest of topics were defined to describe the particular area of concern of the standard, e.g., Profile in the case of SAREF, Sample in the case of SSN or Operation in the case of oneM2M.

In order to check if there were more classes and properties shared by the standards, but not included in the requirements, the shared glossary of terms was analysed. Table 7.20 shows the shared classes among the standards, while Table 7.21 shows the shared properties. In order to improve the readability of the results, both Table 7.20 and Table 7.21 only include the information shared by more than one ontology.

Table 7.20: Classes shared by the analysed standards

\begin{tabular}{|l|l|}
\hline Class & Standards that contain the class \\
\hline ActuatingFunction & SAREF, oneM2M (as ControllingFunction) \\
\hline Actuator & ISO/IEC 30141, SAREF, SSN \\
\hline Command & oneM2M, SAREF \\
\hline Device & ISO/IEC 30141, OCF, oneM2M, SAREF \\
\hline Function & oneM2M, SAREF \\
\hline Measurement & SAREF, SSN (as Observation) \\
\hline Network & ISO/IEC 30141, SAREF \\
\hline Property & OCF, oneM2M, SAREF, SSN \\
\hline Service & ISO/IEC 30141, oneM2M, SAREF \\
\hline Thing & ISO/IEC 30141, oneM2M, SSN (as Feature of Interest) \\
\hline Sensor & ISO/IEC 30141, SAREF, SSN \\
\hline State & OCF, SAREF, \\
\hline SensingFunction & oneM2M (as MeasuringFunction), SAREF \\
\hline
\end{tabular}

From Tables 7.19, 7.20 and 7.21 it can be observed that in this scenario the majority of the shared requirements were related to the definition of classes and also to relations, while restrictions such as cardinalities are not shared. Consequently, the variant-domain knowledge according to these IoT standards is related to the definition of classes and relations.

Figure 7.19 shows the classes shared by more than one standards. From this figure it can be observed that some of the standards are related to generic terms, e.g., a thing, while other standards describe more specific aspects of the domain, e.g., commands associated to functions performed by devices. 
Table 7.21: Properties shared by the analysed standards

\begin{tabular}{|l|l|}
\hline Property & Standards that contain the property \\
\hline consistsOf & OCF, SAREF, oneM2M \\
\hline hosts & OCF, SSN \\
\hline hasCommand & oneM2M,SAREF \\
\hline hasFunction & oneM2M, SAREF \\
\hline hasInput & oneM2M, SSN, \\
\hline hasManufacturer & OCF, SAREF, \\
\hline hasOutput & oneM2M, SSN \\
\hline hasProperty & OCF, SSN \\
\hline hasValue & oneM2M, SAREF \\
\hline offers & oneM2M, SAREF \\
\hline represents & ISO/IEC 30141, SAREF, oneM2M \\
\hline
\end{tabular}

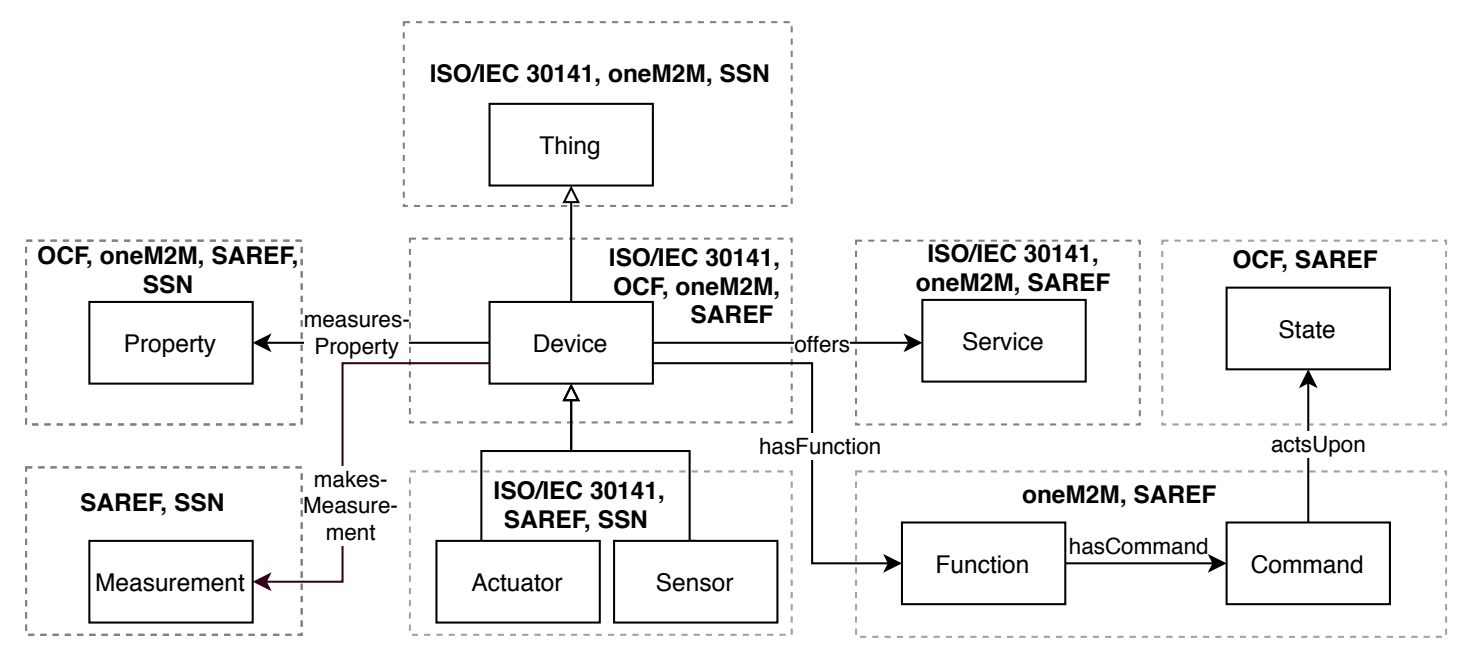

Figure 7.19: Overview of the shared terms between standards

\subsubsection{Conclusions of the experiments}

With respect to the hypothesis and based on the information retrieved in the experiments, the following conclusions can be outlined:

- H3. The majority of functional requirements shared by a set of ontologies from the same domain are related to the definition of terms and to the existence of relations between classes, while more restrictive requirements such as cardinalities are not frequently shared.

The analysis of conformance in these experiments shows how ontologies and standards in a particular domain share several requirements, although they were 
mostly related to the definition of classes. Those restrictive requirements, e.g., those that lead to cardinality axioms in the ontology, were only satisfied by the ontology or standard from which they were extracted. In addition, it was observed that the analysed standards in the IoT domain (i.e., the SAREF ontology, the SSN ontology, the oneM2M ontology, the ISO/IEC 30141 and the OCF standard) did not share any minimum commitment between all of them, although some of them shared some terms and requirements. These results show that even though these IoT standards are related to the same domain, they were created to provide support to different areas of concern in the same IoT field and, therefore, there is a minimum overlap between them. With all this information, it was concluded that hypothesis $\mathrm{H} 3$ is true. 



\section{Chapter 8}

\section{Conclusions and future work}

As stated in Chapter 3, the overall objective of this thesis is to advance the current state of the art in the ontology verification field. To that end, this thesis aims at helping ontology engineers and practitioners to verify their ontologies regarding their ontological requirements by proposing an ontology testing method. This thesis also intends to extract information from the artefacts generated and used during the verification process in order to support making decisions and monitoring ontology development. Furthermore, this thesis also aims at automating the conformance analysis between an ontology and a standard by means of the verification of the requirements that this standard imposes. The objectives of this thesis are fulfilled by the main contributions of the thesis:

- Ontology testing method for ontology verification. This method considers the involvement and feedback of domain experts and users regarding the verification process. To define the tests, a testing language based on lexico-syntactic patterns is proposed as part of this testing framework. This testing language was proposed after an analysis of how requirements are specified, which facilitates the definition of the tests during the verification process (Section 7.1, H1). The results of the testing process are stored in a machine-readable format, which also provides traceability between the requirements, the tests and the ontology implementation.

From the analysis of the requirements specification it was confirmed that there are several polysemous expressions in requirements that could result in different ontology structures. Therefore, these requirements could also result in different ontology models and the direct translation from requirements to an ontology is unfeasible. This fact illustrates the need for improvement in the specification of requirements, which should aim to avoid ambiguities and to be more precise in order to identify the appropriate needs to be modelled. More precise requirements 
would make possible to reduce errors during the modelling of ontologies and also to ease the automation from requirements into tests.

Significant difficulties to find available real-world requirements were found, which hindered the analysis of the requirements specifications and, consequently, limited the defined testing language. Publishing ontology requirements online would help the analysis of their specification and, therefore, would facilitate research related to their definition, formalisation and verification.

Several testing techniques in Ontology Engineering are inspired by Software Engineering, which is a more mature field where research on testing approaches has been active for decades. Therefore, the adoption of software testing techniques, such as keyword-driven testing or test-driven development, which are widely integrated into the software development process, facilitates the integration of testing activities into the Ontology Engineering field.

- The online tool Themis to support the testing process. Themis allows to execute tests on one or multiple ontologies written following the aforementioned testing language and to store the test suite in a machine-readable file, facilitating its reusability and publication. Since Themis is an online web application it does not require any installation process. Moreover, it includes mechanisms to guide the users throughout the definition of tests.

As shown in Section 7.1, Themis reduced errors during the verification process for those users that are familiar with the OWL language, as well as for developers experts in it, in comparison with Protégé. However, the results obtained during the evaluation of the testing process presented in Section 7.1 also raised the question of who should be able to verify an ontology and to which type of users the testing process should be oriented to. Users without any ontology background found difficult to understand the restrictions that can be described in an ontology. Consequently, it is difficult for them to go beyond asking for the presence of classes and properties, which is not enough for the verification process.

Moreover, ontology visualisation or examples of use are needed by them to understand the structure of the ontology. However, domain experts and users that have knowledge about ontologies, even if they are not experts, managed to verify an ontology using tests and to analyse the restrictions included in it. Ontology verification testing processes should consider ontology engineers and practitioners with a minimum of knowledge regarding ontologies as potential users, since a manual revision of the verification status and tests is needed. 
During the experiment, it was also found that all types of participants made mistakes during the verification process, even those that are experts in the OWL language. These mistakes refer to incorrect answers, i.e., to affirm that a requirement is satisfied by an ontology when it is not or vice versa. Such incorrect answers can affect the development of the ontology, since the verification process helps to ensure that all the expected requirements are satisfied. Therefore, this fact reinforces the need for testing approaches that should be used by ontology engineers in order to develop complete ontologies according to their associated requirements.

Up to now, Themis was integrated into several research projects and tools, namely: (1) the VICINITY H2020 project ${ }^{57}$, where it was used to verify that all the requirements expected by domain experts were satisfied, and that the ontology network was conformant with the expected standard ontologies; (2) the DELTA H2020 project ${ }_{58}^{5}$ where it was used to allow domain experts to execute tests on the DELTA ontology network, and was used in order to report that all the requirements identified by the domain experts were satisfied; (3) the Activage H2020 project, ${ }_{59}$ where it was integrated into the ontology maintenance tool, in order to help developers to check whether new requirements provided by users and domain experts are already covered by the ontology; (4) the OnToology tool (Alobaid et al., 2019), which integrates Themis in order to execute tests every time the associated ontology is changed, performing continuous integration and ensuring that the requirements are still satisfied although the ontology changes; and (5) the ETSI SAREF portal ${ }^{60}$ where Themis is integrated into the development pipeline to execute tests every time a modification to the ontologies is performed.

Themis was used not only for verifying that at some point an ontology satisfies its requirements, but also in continuous integration to ensure that the ontology still satisfies the requirements while it evolves. Moreover, the testing method was also used during the maintenance activity of an ontology development process, helping developers to check what is the new knowledge that should be added to an ontology. These scenarios reflect the benefits of having ontology requirements throughout the ontology development process.

- A set of ontology metrics derived from the verification process. The re-

57 https://www.vicinity2020.eu/vicinity

58 https://www.delta-h2020.eu

59 https: //www.activageproject.eu

60 https://saref.etsi.org/ 
sults of the verification process should be integrated into the development process in order to monitor the status of the ontology and its development. To that end, this thesis presents a set of metrics that goes beyond the ontology implementation itself, related to the artefacts involved in the verification process. Although this thesis does not identify all possible metrics that could be extracted from such artefacts, it defines some of those that are relevant for the identified needs of each role involved in the development process.

The analysis between the effects of different metrics related to the verification activity provided, in addition to the relations between metrics (Section 7.2, H2), insights about the ontology development process that could help predict several characteristics related to it. For example, it was found that the complexity of requirements does influence the time of the development. This information should be considered by ontology engineers at the first steps of the development processes to improve the management of them.

Moreover, the interpretation of the metrics depends on the use case in which they are used. As an example, the interpretation of the size of the ontology can vary depending on the aspect to be analysed: in the case of completeness the more axioms the better, while in the case of understandability the fewer axioms the better.

The methodology followed in the experiment did not allow to have an automated way to calculate several metrics, such as the Pending Formalised Requirements Percentage (PendFReqPtc) one, which would measure the percentage of requirements that are included in the ORSD as pending requirements but that are already included in the test suite and implemented in the ontology. To be able to automate this calculation, it is needed to have in every sprint all the requirements formalised in the test suite. The more information available in the sprints, the more metrics to be calculated and, therefore, the more information available related to the development process.

- A conformance testing method through ontology requirements inspired by software engineering techniques. The adoption of conformance analyses in the Ontology Engineering field could help to ensure the quality of ontologies by verifying whether they conform to well-known standards. Currently, standardisation bodies develop and publish standard ontologies, but they do not provide any method or technique to analyse conformance. As it is recommended by the W3C (Dubost et al., 2005), every specification of a standard should specify the 
conformance clauses and provide a method to analyse it.

While the experimentation related to the conformance approach is oriented only to the IoT domain, which limits the generalisation of the results, it provides insights about the most frequent type of shared requirements among a set of ontologies and standards, i.e., those related to the definition of classes and to the definition of the properties between them (Section 7.3, H3). Ontology engineers and practitioners should take this into account when specifying their requirements. Currently, requirements related only to the definition of classes are not usually included in the specification of requirements.

Moreover, a minimum common knowledge identification method derived from the conformance method is also described in this thesis. It aims at analysing the conformance among a set of standards related to the same domain, identifying the knowledge that is common between them. This method allows being aware of how a set of standards covers their domain, identifying their focus, their similarities and their differences, as well as whether there are conflicts between them.

Results show that although the analysed standards were related to the same domain, i.e., IoT, they were created to provide support to different areas of concern. Therefore, there is a minimum overlap between them. These results demonstrate that the coexistence of several standards related to the same domain does not necessarily mean that they duplicate their definitions of terms (Section 7.3, H3). Furthermore, the analysis of the common knowledge based on their requirements provides an overview of the overlaps and conflicts related to the standards of the same domain.

Ontology engineers and practitioners should consider that it is important to spend effort during the definition of ontology requirements; they can be useful not only for ontology verification, but also for ontology management since metrics can be extracted from them, and for ontology conformance. The more quality in the definition of these requirements, the more precise results in these scenarios.

Even though this thesis presents advances to the ontology verification field, there are still some open problems that have not been addressed in this thesis, or that have come out as a consequence of the advances proposed in it. These problems encompass the following lines of future work, which are grouped by contribution:

- Regarding the first contribution of the thesis, which is the ontology testing method for ontology verification, the lines of future work include the following: 
- Extension to requirements. The set of requirements gathered by this thesis is limited to available functional ones written in the English language. One line of future work includes the extension of the corpus with requirements written in other languages, e.g., in Spanish. This extension would improve the analysis of the requirements specification, lead to the analysis of more types of requirements and, consequently, to richer testing languages.

- Implementation of a recommendation system for the translation of requirements into tests. One of the main challenges in the verification process is to translate from natural language requirements into tests. Currently, this translation is manual, although guidelines and recommendations are provided. However, a system to recommend potential tests based on requirements could be implemented, considering that due to the ambiguity of requirements, the same requirement could be translated into several tests. This recommendation system could reduce the time spent in the definition of tests.

- Regarding the second contribution of the thesis, which is the online tool Themis, the lines of future work include the following:

- Improvement of the usability of Themis. During the experimentation, participants' feedback illustrated the importance of guidelines for defining tests, especially when working with testing languages. Therefore, in order to improve the usability of Themis and also to ease the verification process for ontology engineers and practitioners, more guidelines and improved syntax checkers could be added.

- Improvement of Themis to gather feedback from users. Some experienced users might be interested in providing their own test expressions in order to support their needs. Therefore, another line of future work consists of enabling the users of Themis the possibility to provide inputs in terms of new test expressions that will be subsequently analysed to check whether they can be added to the testing framework.

- Extension of Themis functionality. Another line of future work consists of extending Themis functionality to allow ontology repair based on the testing results. With this, Themis will be able not only to identify conflicts in an ontology, but also to add the needed axioms to solve them.

- Regarding the third contribution of the thesis, which is the set of ontology met- 
rics derived from the verification process, the lines of future work include the following:

- Improvement of the analysis of the ontology development process. The metrics proposed in this thesis were extracted from the requirements, the tests and the ontology implementation. Further research regarding metrics can be oriented to the identification of metrics related to other artefacts in the development process, e.g., the ontology documentation, and to analyse more effects between them. Moreover, in this thesis only the verification and implementation processes are supported by metrics. However, future work can be directed to the definition of metrics related to the extraction of requirements, or to the generation of the ontology documentation. Information gathered from more metrics could help to provide more insights to improve ontology development management.

- Improvement of the precision of the metrics used for the analysis of the ontology development process. The information obtained with the ontology metrics about development time depended on a third-party tool, i.e., GitHub. Moreover, the developers only used the master branch to submit their work and they were not asked to measure the time spent, since the tool does not allow to do that. Consequently, the results regarding the development time are approximated values. Moreover, the time could only be calculated in days and, therefore, the precision of the results was not as accurate as expected. A tool that stores all this information related to the development time would allow improving the precision of the metrics.

- Diagnosis of the development process status. Another line of future work includes the proposal of a unique metric that combines data obtained from the available artefacts with the aim of providing ontology engineers with a measurement of the health condition of the ontology development process. This health condition would refer to the quality of the process considering all the available artefacts. An ideal situation in ontology development includes a low number of pending requirements, a high level of formalised requirements coverage and a high level of test coverage. This ideal situation indicates in a particular sprint that the ontology is complete (or almost complete) regarding the requirements defined by the domain experts, that the ontology test suite covers a high percentage of the requirements identified in the ontology requirements specification document, and that the ontology implementation 
passed a high percentage of the tests defined in the test suite.

- Finally, regarding the fourth contribution of the thesis, which is the conformance method through ontology requirements, the lines of future work include the following:

- Extension of the evaluation domain for conformance analysis. In this thesis the conformance testing method was applied in the IoT domain. However, future work includes performing evaluations that consider other domains. These evaluations would allow gathering more information related to the overlaps and inconsistencies of an ontology and a standard that belong to the same domain.

- New process for certification of ontologies. Based on the conformance testing method, and following software engineering practices, another line of future work is also directed to the adoption of certification practices in the Ontology Engineering field, in order to propose techniques to issue certificates for ontologies based on their conformance with standards. This process would assure ontology engineers and practitioners that a particular ontology version has conformance with a standard. 


\section{Bibliography}

Aguado De Cea, G., Gómez-Pérez, A., Montiel-Ponsoda, E., and Suárez-Figueroa, M. C. (2008). Natural language-based approach for helping in the reuse of ontology design patterns. In Proceedings of the 16th International Conference on Knowledge Engineering and Knowledge Management, Acitrezza, Italy, September 29 - October 2, 2008, pages 32-47. Springer. 72

Alobaid, A., Garijo, D., Poveda-Villalón, M., Santana-Perez, I., Fernández-Izquierdo, A., and Corcho, O. (2019). Automating ontology engineering support activities with OnToology. Journal of Web Semantics, 57. Elsevier. 175

Baader, F., Horrocks, I., and Sattler, U. (2008). Description logics. Foundations of Artificial Intelligence, 3:135-179. Elsevier. 75, 85, 111, 147

Basilevsky, A. (2009). Statistical factor analysis and related methods: theory and applications. John Wiley and Sons. 141

Beaver, D. I. (1997). Presupposition. In Handbook of logic and language, pages 9391008. Elsevier. 37

Beck, K. (1999). Extreme Programming. Addison-Wesley. 16

Beck, K. (2003). Test-driven development: by example. Addison-Wesley Professional. 18,45

Beck, K. and Gamma, E. (2000). Extreme programming explained: embrace change. Addison-Wesley Professional. 32, 34

Begier, B. (2010). Users' involvement may help respect social and ethical values and improve software quality. Information Systems Frontiers, 12(4):389-397. Springer. 3 
Bertolino, A. (2007). Software testing research: Achievements, challenges, dreams. In Proceedings of the Workshop on the Future of Software Engineering, Minneapolis, USA, May 23-25, 2007, pages 85-103. IEEE Computer Society. 16

Bezerra, C., Freitas, F., and Santana, F. (2013). Evaluating ontologies with competency questions. In Proceedings of the IEEE/WIC/ACM International Conferences on Web Intelligence and Intelligent Agent Technology, Atlanta, Georgia, USA, November 17-20, 2013, volume 3, pages 284-285. IEEE Computer Society. 71

Bezerra, C., Santana, F., and Freitas, F. (2014). CQChecker: A Tool to Check Ontologies in OWL-DL using Competency Questions written in Controlled Natural Language. Learning and Nonlinear Models, 12(2):115-129. SBRN. xv, 41,42

Bhat, T. and Nagappan, N. (2006). Evaluating the efficacy of test-driven development: industrial case studies. In Proceedings of the 2006 ACM/IEEE International Symposium on Empirical Software Engineering, Rio de Janeiro, Brazil, September 21-22, 2006, pages 356-363. ACM. xv, 19

Blomqvist, E., Sepour, A. S., and Presutti, V. (2012). Ontology testing-methodology and tool. In Proceedings of the 18th International Conference on Knowledge Engineering and Knowledge Management, Galway City, Ireland, October 8-12, 2012, pages 216-226. Springer. Xv, 3, 40, 41, 67

Boehm, B., Clark, B., Horowitz, E., Westland, C., Madachy, R., and Selby, R. (1995). Cost models for future software life cycle processes: COCOMO 2.0. Annals of software engineering, 1(1):57-94. Springer. 24

Brickley, D. and Guha, R. V. (2014). RDF Schema 1.1. W3C Recommendation. Available at: https://www.w3.org/TR/rdf-schema/, 100

Costello, R. J. and Liu, D.-B. (1995). Metrics for requirements engineering. Journal of Systems and Software, 29(1):39-63. Elsevier. 26, 27, 103

Cuenca, J., Larrinaga, F., and Curry, E. (2019). Experiences on applying SPL Engineering Techniques to Design a (Re) usable Ontology in the Energy Domain. In Proceedings of 31st International Conference on Software Engineering and Knowledge Engineering, Lisbon, Portugal, July 10-12, 2019. KSI Research Inc. 119

Daga, E., Blomqvist, E., Gangemi, A., Montiel-Ponsoda, E., Nikitina, N., Presutti, V., and Villazón-Terrazas, B. (2010). NeOn D2. 5.2 Pattern based ontology design: 
methodology and software support. Technical report. NeOn Project. http:// Www.neon-project.org, 74, 76, 77

Dahlström, E., Dengler, P., Grasso, A., Lilley, C., mcCormack, C., Schepers, D., Watt, J., Ferraiolo, J., Jun, F., and Jackson, D. (2011). Scalable Vector Graphics (SVG) 1.1 Specification. W3C Recommendation. Available at: https://www.w3.org/ TR/SVG11/, 23

Dardailler, D. (2002). Conformance Testing and Certification Model for W3C Specifications. W3C Note. Available at: https://www.w3.org/QA/2002/01/ Note-qa-certif-20020102.html. 67.

Davies, K., Keet, C. M., and Ławrynowicz, A. (2017). TDDonto2: A Test-Driven Development Plugin for arbitrary TBox and ABox axioms. In Proceedings of the 14th European Semantic Web Conference, Portoroz, Slovenia, May 28- June 1, 2017, pages 120-125. Springer. 46

Davis, A., Overmyer, S., Jordan, K., Caruso, J., Dandashi, F., Dinh, A., Kincaid, G., Ledeboer, G., Reynolds, P., Sitaram, P., Ta, A., and Theofanos, M. (1993). Identifying and measuring quality in a software requirements specification. In Proceedings of the 1st International Software Metrics Symposium, Baltimore, Maryland, USA, May 21-22, 1993, pages 141-152. IEEE Computer Society. 25, 104

De Nicola, A., Missikoff, M., and Navigli, R. (2005). A proposal for a unified process for ontology building: UPON. In Proceedings of the 16th International Conference on Database and Expert Systems Applications, Copenhagen, Denmark, August 22-26, 2005, pages 655-664. Springer. 99

Debruyne, C., Tran, T.-K., and Meersman, R. (2013). Grounding ontologies with social processes and natural language. Journal on Data Semantics, 2(2-3):89-118. Springer. 99

Dennis, M., van Deemter, K., Dell'Aglio, D., and Pan, J. Z. (2017). Computing Authoring Tests from Competency Questions: Experimental Validation. In Proceedings of the 16th International Semantic Web Conference, Vienna, Austria, October 21-25, 2017, pages 243-259. Springer. 72, 79

Djedidi, R. and Aufaure, M. (2010). ONTO-EVO ${ }^{A} L$ an Ontology Evolution Approach Guided by Pattern Modeling and Quality Evaluation. In Proceedings of the 6th International Symposium on Foundations of Information and Knowledge Systems, Sofia, Bulgaria, February 15-19, 2010, pages 286-305. Springer-Verlag. 2 
Dubost, K., Rosenthal, L., Hazaël-Massieux, D., and Henderson, L. (2005). QA Framework: Specification Guidelines. W3C Recommendation. Available at: https: //www.w3.org/TR/qaframe-spec/, 23, 176

Duque-Ramos, A., Fernández-Breis, J. T., Iniesta, M., Dumontier, M., Aranguren, M. E., Schulz, S., Aussenac-Gilles, N., and Stevens, R. (2013). Evaluation of the OQuaRE framework for ontology quality. Expert Systems with Applications, 40(7):2696-2703. Elsevier. 47

Duque-Ramos, A., Fernández-Breis, J. T., Stevens, R., and Aussenac-Gilles, N. (2011). OQuaRE: A SQuaRE-based approach for evaluating the quality of ontologies. Journal of Research and Practice in Information Technology, 43(2):159-179. Australian Computer Society Inc. 1, 49

ETSI (2020). ETSI TS 103264 V3.1.1. SmartM2M; Smart Applications; Reference Ontology and oneM2M Mapping. Technical report. 74, 116, 160

Fenton, N. and Bieman, J. (1997). Software metrics: a rigorous and practical approach. PWS Publishing Company. 24, 26, 27, 105

Fenton, N. E. and Neil, M. (2000). Software metrics: roadmap. In Proceedings of the Conference on the Future of Software Engineering, Limerick, Ireland, June 04 11, 2000, pages 357-370. ACM. 2, 24, 67, 95

Fernández-Izquierdo, A., Poveda-Villalón, M., and García-Castro, R. (2019). CORAL: A Corpus of Ontological Requirements Annotated with Lexico-Syntactic Patterns. In Proceedings of 16th Extended Semantic Web Conference, Portorož, Slovenia, June 2-6, 2019, pages 443-458. Springer. 77

Fernández-López, M., Gómez-Pérez, A., and Juristo, N. (1997). Methontology: from ontological art towards ontological engineering. In Proceedings of the Ontological Engineering AAAI97 Spring Symposium Series, Stanford University, EEUU, March 24-26,1997, pages 33-40. 1. 100

Gangemi, A., Catenacci, C., Ciaramita, M., and Lehmann, J. (2006). Modelling ontology evaluation and validation. In Proceedings of the 3rd European Semantic Web Conference, Budva, Montenegro, June 11-14, 2006, pages 140-154. SpringerVerlag. 2, 48, 49, 67

Gangemi, A. and Presutti, V. (2009). Ontology design patterns. In Handbook on ontologies, pages 221-243. Springer. 74, 82, 114, 153 
García-Castro, R. (2009). Benchmarking semantic web technology, volume 3 of Studies on the Semantic Web. IOS Press. 52

García-Ramos, S., Otero, A., and Fernández-López, M. (2009). OntologyTest: A tool to evaluate ontologies through tests defined by the user. In Proceedings of the 10th International Work-Conference on Artificial Neural Networks on Artificial Neural Networks, Salamanca, Spain, June 10-12, 2009, pages 91-98. Springer. 43,67

Gómez-Pérez, A., Corcho, O., and Fernández-López, M. (2004). Ontological Engineering: with examples from the areas of Knowledge Management, e-Commerce and the Semantic Web. Springer. 1

Gómez-Pérez, A., Juristo, N., and Pazos, J. (1995). Evaluation and assessment of knowledge sharing technology. In Towards Very Large Knowledge Bases, pages 289-296. IOS Press. 1

Grabowski, J., Hogrefe, D., Réthy, G., Schieferdecker, I., Wiles, A., and Willcock, C. (2003). An introduction to the testing and test control notation (TTCN-3). Computer Networks, 42(3):375-403. Elsevier. 22

Graydon, P., Habli, I., Hawkins, R., Kelly, T., and Knight, J. (2012). Arguing conformance. IEEE software, 29(3):50-57. IEEE. 2, 20

Grüninger, M. and Fox, M. S. (1995). Methodology for the design and evaluation of ontologies. In Proceedings of the Workshop on Basic Ontological Issues in Knowledge Sharing. $\mathrm{xv}, 1,30,72$

Guarino, N. (2004). Toward a formal evaluation of ontology quality. IEEE intelligent Systems, 19(4):78-79. IEEE Computer Society. 1

Guarino, N. and Welty, C. A. (2009). An overview of OntoClean. In Handbook on Ontologies, pages 201-220. Springer. 1

Gunning, R. (1952). The Technique of Clear Writing. McGraw-Hill, New York. 27

Halilaj, L., Petersen, N., Grangel-González, I., Lange, C., Auer, S., Coskun, G., and Lohmann, S. (2016). Vocol: An integrated environment to support versioncontrolled vocabulary development. In Proceedings of the 20th International Conference on Knowledge Engineering and Knowledge Management, Bologna, Italy, November 19-23, 2016, pages 303-319. Springer. xv, 44 
Haller, A., Janowicz, K., Cox, S. J., Lefrançois, M., Taylor, K., Le Phuoc, D., Lieberman, J., García-Castro, R., Atkinson, R., and Stadler, C. (2019). The modular SSN ontology: A joint W3C and OGC standard specifying the semantics of sensors, observations, sampling, and actuation. Semantic Web, 10(1):9-32. IOS Press. 116

Haller, A., Janowicz, K. V., Cox, S., Le Phuoc, Danh, T. K., Lefrançois, M., Atkinson, R., García-Castro, R., Lieberman, J., and Stadler, C. (2017). Semantic Sensor Network Ontology. W3C Recommendation. Available at: https://www.w3. org/TR/Vocab-ssn/, 160

Heineman, G. T. and Councill, W. T. (2001). Component-based software engineering: Putting the pieces together. Addison-Westley. 44

Hitzler, P., Krötzsch, M., Parsia, B., Patel-Schneider, P. F., and Rudolph, S. (2012). OWL 2 Web Ontology Language Primer. W3C Recommendation. Available at: https://www.w3.org/TR/owl2-primer/, 100

Hristozova, M. and Sterling, L. (2002). An eXtreme method for developing lightweight ontologies. In Proceedings of the Workshop on Ontologies in Agent Systems, 1st International Joint Conference on Autonomous Agents and Multi-Agent Systems, Bologna, Italy, 2002. CEUR Workshop Series. 32,65

Iqbal, S., Naeem, M., and Khan, A. (2012). Yet another Set of Requirement Metrics for Software Projects. International Journal of Software Engineering and Its Applications, 6(1):19-28. Science and Engineering Research Support Society. 26

ISO (1994). ISO/IEC 9646-1:1994 Information technology; Open Systems Interconnection; Conformance testing methodology and framework. Technical report, International Organization for Standardization. 20, 67

ISO (2011a). ISO/IEC 25010:2011 Systems and software engineering; Systems and software Quality Requirements and Evaluation (SQuaRE); System and software quality models. Technical report, International Organization for Standardization. 49

ISO (2011b). ISO/IEC 25040:2011 Systems and software engineering; Systems and software Quality Requirements and Evaluation (SQuaRE); Evaluation process. Technical report, International Organization for Standardization. 14 
ISO (2016). ISO/IEC 25066:2016 Systems and software engineering; Systems and software Quality Requirements and Evaluation (SQuaRE); Common Industry Format (CIF) for Usability; Evaluation Report. Technical report, International Organization for Standardization. 14

ISO (2017). ISO/IEC 30141:2017 Internet of Things (IoT); Reference Architecture. Technical report, International Organization for Standardization. 110, 159, 160

ISO/IEC/IEEE (2013). ISO/IEC/IEEE 29119-1:2013 Software and systems engineering; Software testing. Technical report, International Organization for Standardization. 15

Janzen, D. and Saiedian, H. (2005). Test-driven development concepts, taxonomy, and future direction. Computer, 38(9):43-50. IEEE. 18

Kan, S. H. (2002). Metrics and models in software quality engineering. Addison-Wesley. 24, 25

Kang, Y.-B., Li, Y.-F., and Krishnaswamy, S. (2012). Predicting reasoning performance using ontology metrics. In Proceedings of the 11th International Semantic Web Conference, Boston, USA, November 11-15, 2012, pages 198-214. Springer-Verlag. 48,49

Keet, C. M. and Ławrynowicz, A. (2016). Test-Driven Development of ontologies. In Proceedings of 13th European Semantic Web Conference, Heraklion, Crete, Greece, May 29 - June 2, 2016, pages 642-657. Springer. xv, 3, 45, 46, 66, 100

Kollia, I., Glimm, B., and Horrocks, I. (2011). SPARQL query answering over OWL ontologies. In Proceedings of 8th Extended Semantic Web Conference, Heraklion, Crete, Greece, May 29-June 2, 2011, pages 382-396. Springer. 38

Koskela, L. (2007). Test Driven: Practical TDD and Acceptance TDD for Java Developers. Manning Publications Co. 18

Kristoffersen, F. and Walter, T. (1996). TTCN: Towards a formal semantics and validation of test suites. Computer Networks and ISDN Systems, 29(1):15-47. Elsevier. 20

Kujala, S. (2003). User involvement: a review of the benefits and challenges. Behaviour and information technology, 22(1):1-16. Taylor and Francis. 3 
Lantow, B. (2016). OntoMetrics: Application of On-line Ontology Metric Calculation. In Joint Proceedings of the BIR 2016 Workshops and Doctoral Consortium co-located with 15th International Conference on Perspectives in Business Informatics Research, Prague, Czech Republic, September 14 - 16, 2016. CEUR Workshop series. 49,50

Eawrynowicz, A. and Keet, C. M. (2016). The TDDonto Tool for Test-Driven Development of DL Knowledge bases. In Proceedings of the 29th International Workshop on Description Logics, Cape Town, South Africa, April 22-25, 2016, volume 1577. CEUR Workshop series. 46

Lazar, I., Motogna, S., and Pârv, B. (2010). Behaviour-Driven Development of Foundational UML Components. Electronic Notes in Theoretical Computer Science, 264(1):91-105. 19

Lozano-Tello, A. and Gómez-Pérez, A. (2004). ONTOMETRIC: A method to choose the appropriate ontology. Journal of Database Management, 2(15):1-18. IGI Global. 2

Lund, A. M. (2001). Measuring usability with the use questionnaire. Usability Interface, $8: 2: 3-6.124$

Ma, Y., Jin, B., and Feng, Y. (2010). Semantic oriented ontology cohesion metrics for ontology-based systems. Journal of Systems and Software, 83(1):143-152. Elsevier. 50

Malone, J., Brown, A., Lister, A. L., Ison, J., Hull, D., Parkinson, H., and Stevens, R. (2014). The Software Ontology (SWO): a resource for reproducibility in biomedical data analysis, curation and digital preservation. Journal of biomedical semantics, 5(1):25. BioMed Central. 74

Miller, R. and Collins, C. T. (2001). Acceptance testing. In Proceedings of the XP Universe Conference, Raleigh, USA, July 23-25, 2001. 17

Montiel-Ponsoda, E. (2011). Multilingualism in Ontologies - Building Patterns and Representation Models. LAP Lambert Academic Publishing. 72

Moseley, S., Randall, S., and Wiles, A. (2003). Experience within ETSI of the combined roles of conformance testing and interoperability testing. In Proceedings of the 33rd Conference on European Solid-State Device Research, Estoril, Portugal, September 16-18, 2003, pages 177-189. IEEE. Xv, 2, 20, 21, 22, 67, 107 
Moser, R., Pedrycz, W., and Succi, G. (2008). A comparative analysis of the efficiency of change metrics and static code attributes for defect prediction. In Proceedings of the 30th International Conference on Software Engineering, Leipzig, Germany, May 10 - 18, 2008, pages 181-190. ACM. 24

Noy, N., Rector, A., Hayes, P., and Welty, C. (2006). Defining n-ary relations on the semantic web. W3C Working Group Note, 12(4). Available at: https://www. w3.org/TR/swbp-n-aryRelations/, 153

OCF (2019). OCF Specification 2.0.2. Technical report, Open Connectivity Foundation. Available at: https://openconnectivity.org/developer/ specifications, 160

oneM2M (2016). TS-0012 oneM2M Base Ontology. Technical report. 116

Orme, A. M., Tao, H., and Etzkorn, L. H. (2006). Coupling metrics for ontology-based system. IEEE software, 23(2):102-108. IEEE. 48, 51

Panov, P., Soldatova, L. N., and Džeroski, S. (2016). Generic ontology of datatypes. Information Sciences, 329:900-920. Elsevier. 74

Parkkila, J., Radulovic, F., Garijo, D., Poveda-Villalón, M., Ikonen, J., Porras, J., and Gómez-Pérez, A. (2017). An ontology for videogame interoperability. Multimedia Tools and Applications, 76(4):4981-5000. Springer. 74

Pérez, J., Arenas, M., and Gutierrez, C. (2009). Semantics and Complexity of SPARQL. ACM Transactions on Database Systems, 34(3):16:1-16:45. ACM. 81

Peroni, S. (2016). A simplified agile methodology for ontology development. In Proceedings of the 13th OWL: Experiences and Directions Workshop and 5th OWL reasoner evaluation workshop, Bologna, Italy, November 20, 2016, pages 55-69. Springer. xv, 2, 3, 35, 36, 65, 99

Poveda-Villalón, M., Gómez-Pérez, A., and Suárez-Figueroa, M. C. (2014). OOPS! (OntOlogy Pitfall Scanner!): An On-line Tool for Ontology Evaluation. International Journal on Semantic Web and Information Systems, 10(2):7-34. IGI Global. 1.

Pressman, R. S. (2005). Software engineering: a practitioner's approach. Palgrave Macmillan. 97, 98 
Presutti, V., Daga, E., Gangemi, A., and Blomqvist, E. (2009). eXtreme design with content ontology design patterns. In Proceedings of the Workshop on Ontology Patterns, collocated with the 8th International Semantic Web Conference, Washington D.C., USA, 25 October, 2009. CEUR Workshop series. xv, 33, 34, 65,99

Presutti, V., Gangemi, A., David, S., Aguado de Cea, G., Suárez-Figueroa, M. C., Montiel-Ponsoda, E., and Poveda-Villalón, M. (2008). D2.5.1 A Library of Ontology Design Patterns: reusable solutions for collaborative design of networked ontologies. Technical report. NeOn Project. http://www. neon-project.org. 2

Rahman, F. and Devanbu, P. (2013). How, and why, process metrics are better. In Proceedings of the 35th International Conference on Software Engineering, San Francisco, USA, May 18-26, 2013, pages 432-441. IEEE Press. 27

Ren, Y., Parvizi, A., Mellish, C., Pan, J. Z., Van Deemter, K., and Stevens, R. (2014). Towards competency question-driven ontology authoring. In Proceedings of the 11th European Semantic Web Conference, Crete, Greece, May 25-29, 2014, pages 752-767. Springer. xix, 3, 36, 38, 66, 72

Runeson, P. (2006). A survey of unit testing practices. IEEE software, 23(4):22-29. IEEE. 16

Schober, D., Tudose, I., Svatek, V., and Boeker, M. (2012). OntoCheck: verifying ontology naming conventions and metadata completeness in Protégé 4. Journal of Biomedical Semantics, 3(S-2):S4. BioMed Central. 51

Sirin, E., Parsia, B., Grau, B. C., Kalyanpur, A., and Katz, Y. (2007). Pellet: A practical OWL-DL reasoner. Journal of Web Semantics, 5(2):51-53. Elsevier. 67

Smullyan, R. R. (2012). First-order logic. Springer. 30

Solis, C. and Wang, X. (2011). A study of the characteristics of behaviour driven development. In Proceedings of the 37th EUROMICRO Conference on Software Engineering and Advanced Applications, Oulu, Finland, August 30 - September 2, 2011, pages 383-387. IEEE. 20

Sommerville, I. (2010). Software Engineering. Addison-Wesley, USA, 9th edition. 24. 25, 27, 67,100

Staab, S., Studer, R., Schnurr, H.-P., and Sure, Y. (2001). Knowledge processes and ontologies. IEEE Intelligent systems, 16(1):26-34. IEEE. xv, 2, 31,65 
Suárez-Figueroa, M. C., Aguado de Cea, G., and Gómez-Pérez, A. (2013). Lights and shadows in creating a glossary about ontology engineering. Terminology, 19(2):202236. John Benjamins Publishing Company. 2, 15

Suárez-Figueroa, M. C., Blomqvist, E., d'Aquin, M., Espinoza, M., Gómez-Pérez, A., Lewen, H., Mozetic, I., Palma, R., Poveda, M., Sini, M., Villazón-Terrazas, B., Zablith, F., and Dzbor, M. (2009a). D5.4.2 Revision and Extension of the NeOn Methodology for Building Contextualized Ontology Networks. Technical report. NeOn Project. http://www.neon-project.org. 33

Suárez-Figueroa, M. C., Brockmans, S., Gangemi, A., Gómez-Pérez, A., Lehmann, J., Lewen, H., Presutti, V., and Sabou, M. (2007). D5.1.1 NeOn modelling components. Technical report. NeOn Project. http://www.neon-project.org, 75

Suárez-Figueroa, M. C., Gómez-Pérez, A., and Fernández-López, M. (2015). The NeOn Methodology framework: A scenario-based methodology for ontology development. Applied Ontology, 10(2):107-145. IOS Press. 1, 2, 71, 99

Suárez-Figueroa, M. C., Gómez-Pérez, A., and Villazón-Terrazas, B. (2009b). How to write and use the ontology requirements specification document. In Proceedings of the International Conference on On the Move to Meaningful Internet Systems, ilamoura, Portugal, November 1-6, 2009, pages 966-982. Springer. 7, 71, 99

Tartir, S., Arpinar, I. B., Moore, M., Sheth, A. P., and Aleman-Meza, B. (2005). OntoQA: Metric-based ontology quality analysis. In Proceedings of IEEE Workshop on Knowledge Acquisition from Distributed, Autonomous, Semantically Heterogeneous Data and Knowledge Sources at 2005 IEEE International Conference on Data Mining, Houston, USA, November 27, 2005, page 45-53. 50, 51, 67

Utting, M. and Legeard, B. (2010). Practical model-based testing: a tools approach. Elsevier. 17

Vrandečić, D. and Gangemi, A. (2006). Unit tests for ontologies. In Proceedings of the 2006 International Conference on On the Move to Meaningful Internet Systems, Montpellier, France, October 29 - November 3, 2006, pages 1012-1020. Springer. 33

Vrandečić, D. and Sure, Y. (2007). How to design better ontology metrics. In Proceedings of the 4th European Semantic Web Conference, Innsbruck, Austria, June 3-7, 200\%, pages 311-325. Springer. 95 
Wieczorek, S., Kozyura, V., Schur, M., and Roth, A. (2012). Practical model-based testing of user scenarios. In Proceedings of the 2012 IEEE International Conference on Industrial Technology, Athens, Greece, March 19-21, 2012, pages 306-311. IEEE. 17

Wiśniewski, D., Potoniec, J., Ławrynowicz, A., and Keet, C. M. (2019). Analysis of Ontology Competency Questions and their formalizations in SPARQL-OWL. Journal of Web Semantics, 59:100534. Elsevier. 3, 38

Wynne, M., Hellesoy, A., and Tooke, S. (2017). The cucumber book: behaviour-driven development for testers and developers. Pragmatic Bookshelf. 20

Yao, H., Orme, A. M., and Etzkorn, L. (2005). Cohesion metrics for ontology design and application. Journal of Computer science, 1(1):107-113. Science Publications. 47, 48,49

Zemmouchi-Ghomari, L. and Ghomari, A. R. (2013). Translating natural language competency questions into SPARQLQueries: a case study. In Proceedings of the First International Conference on Building and Exploring Web Based Environments, Seville, Spain, January 27-February 1, 2013, pages 81-86. IARIA XPS Press. 72

Zhe, Y., Zhang, D., and Chuan, Y. (2006). Evaluation metrics for ontology complexity and evolution analysis. In Proceedings of the IEEE International Conference on eBusiness Engineering, Shanghai, China, October 24-26, 2006, pages 162-170. IEEE Computer Society. 48, 49, 50, 103 


\section{ANNEX A}

\section{Test implementation catalogue}

Table A.1: T1. Class A exists

\begin{tabular}{|c|c|c|c|}
\hline Goal: & \multicolumn{3}{|l|}{ T1. Class A exists } \\
\hline Test expression: & \multicolumn{3}{|l|}{ [ClassA] type Class } \\
\hline Type: & Simple test & Related to: & Classes \\
\hline \multicolumn{2}{|c|}{ Test precondition } & \multicolumn{2}{|c|}{ Test preparation } \\
\hline \multicolumn{2}{|l|}{ Class A exists } & \multicolumn{2}{|l|}{-} \\
\hline \multicolumn{4}{|c|}{ Assertions to test the ontology behaviour } \\
\hline \multicolumn{2}{|r|}{ Axiom } & \multicolumn{2}{|c|}{ Result } \\
\hline \multicolumn{2}{|r|}{-} & \multicolumn{2}{|l|}{-} \\
\hline
\end{tabular}

Table A.2: T2. Subsumption relation between classes A and B

\begin{tabular}{|c|c|c|c|}
\hline Goal: & \multicolumn{3}{|c|}{ T2. Subsumption relation between classes A and B } \\
\hline Test expression: & \multicolumn{3}{|c|}{ [ClassA] subClassOf [ClassB] } \\
\hline Type: & Simple test & Related to: & Classes \\
\hline \multicolumn{2}{|c|}{ Test preconditio } & \multicolumn{2}{|c|}{ Test preparation } \\
\hline \multicolumn{2}{|c|}{ Class A and Class B exist } & \multicolumn{2}{|c|}{$\begin{array}{l}\text { (S 1.1) Declaration of } \neg \mathrm{A} \\
\text { (S 1.2) Declaration of } \neg \mathrm{B}\end{array}$} \\
\hline \multicolumn{4}{|c|}{ Assertions to test the ontology behaviour } \\
\hline \multicolumn{2}{|r|}{ Axiom } & \multicolumn{2}{|c|}{ Result } \\
\hline \multicolumn{2}{|c|}{ (S 2) Assertion $\mathrm{A}^{\prime} \sqsubseteq \neg \mathrm{A} \sqcap \mathrm{B}$} & \multicolumn{2}{|c|}{ Consistent ontology } \\
\hline \multicolumn{2}{|c|}{ (S 3) Assertion $\mathrm{A}^{\prime} \sqsubseteq \mathrm{A} \sqcap \neg \mathrm{B}$} & \multicolumn{2}{|c|}{ Unsatisfiable class } \\
\hline \multicolumn{2}{|c|}{ (S 4) Assertion $\mathrm{A}^{\prime} \sqsubseteq \mathrm{A} \sqcap \mathrm{B}$} & \multicolumn{2}{|c|}{ Consistent ontology } \\
\hline
\end{tabular}


Table A.3: T3. Disjointness between two classes A and B

\begin{tabular}{|c|c|c|c|}
\hline Goal: & \multicolumn{3}{|c|}{ T3. Disjointness between two classes A and B } \\
\hline Test expression: & \multicolumn{3}{|c|}{ [ClassA] disjointWith [ClassB] } \\
\hline Type: & Simple test & Related to: & Classes \\
\hline \multicolumn{2}{|c|}{ Test preconditior } & \multicolumn{2}{|c|}{ Test preparation } \\
\hline \multicolumn{2}{|c|}{ Class A and Class B exist } & \multicolumn{2}{|c|}{$\begin{array}{l}\text { (D 1.1) Declaration of } \neg A \\
\text { (D 1.2) Declaration of } \neg B\end{array}$} \\
\hline \multicolumn{4}{|c|}{ Assertions to test the ontology behaviour } \\
\hline \multicolumn{2}{|r|}{ Axiom } & \multicolumn{2}{|c|}{ Result } \\
\hline \multicolumn{2}{|c|}{ (D 2) Assertion $\mathrm{A}^{\prime} \sqsubseteq \neg \mathrm{A} \sqcap \mathrm{B}$} & \multicolumn{2}{|c|}{ Consistent ontology } \\
\hline \multicolumn{2}{|c|}{ (D 3) Assertion $\mathrm{A}^{\prime} \sqsubseteq \mathrm{A} \sqcap \neg \mathrm{B}$} & \multicolumn{2}{|c|}{ Consistent ontology } \\
\hline \multicolumn{2}{|c|}{ (D 4) Assertion $A^{\prime} \sqsubseteq A \sqcap \mathrm{B}$} & \multicolumn{2}{|c|}{ Unsatisfiable class } \\
\hline
\end{tabular}

Table A.4: T4. Equivalence between two classes A and B

\begin{tabular}{|c|c|c|c|}
\hline Goal: & \multicolumn{3}{|c|}{ T4. Equivalence between two classes $\mathrm{A}$ and $\mathrm{B}$} \\
\hline Test expression: & \multicolumn{3}{|c|}{ [ClassA] equivalentTo [ClassB] } \\
\hline Type: & Simple test & Related to: & Classes \\
\hline \multicolumn{2}{|c|}{ Test preconditio } & \multicolumn{2}{|c|}{ Test preparation } \\
\hline \multicolumn{2}{|c|}{ Class A and Class B exist } & \multicolumn{2}{|c|}{$\begin{array}{l}\text { (E 1.1) Declaration of } \neg \mathrm{A} \\
\text { (E 1.2) Declaration of } \neg \mathrm{B}\end{array}$} \\
\hline \multicolumn{4}{|c|}{ Assertions to test the ontology behaviour } \\
\hline \multicolumn{2}{|r|}{ Axiom } & \multicolumn{2}{|c|}{ Result } \\
\hline \multicolumn{2}{|c|}{ (E 2) AssertionA' $\sqsubseteq \neg \mathrm{A} \sqcap \mathrm{B}$} & \multicolumn{2}{|c|}{ Unsatisfiable class } \\
\hline \multicolumn{2}{|c|}{ (E 3) Assertion $\mathrm{A}^{\prime} \sqsubseteq \mathrm{A} \sqcap \neg \mathrm{B}$} & \multicolumn{2}{|c|}{ Unsatisfiable class } \\
\hline \multicolumn{2}{|c|}{ (E 4) Assertion $A^{\prime} \sqsubset A \sqcap \mathrm{B}$} & \multicolumn{2}{|c|}{ Consistent ontology } \\
\hline
\end{tabular}

Table A.5: T5. Property P exists

\begin{tabular}{|c|c|c|c|}
\hline Goal: & \multicolumn{3}{|l|}{ T5. Property $\mathrm{P}$ exists } \\
\hline Test expression: & \multicolumn{3}{|l|}{ [PropertyP] type Property } \\
\hline Type: & Simple test & Related to: & Property \\
\hline \multicolumn{2}{|c|}{ Test precondition } & \multicolumn{2}{|c|}{ Test preparation } \\
\hline \multicolumn{2}{|l|}{ Property $\mathrm{P}$ exist } & \multicolumn{2}{|l|}{-} \\
\hline \multicolumn{4}{|c|}{ Assertions to test the ontology behaviour } \\
\hline \multicolumn{2}{|r|}{ Axiom } & \multicolumn{2}{|c|}{ Result } \\
\hline \multicolumn{2}{|r|}{-} & \multicolumn{2}{|l|}{-} \\
\hline
\end{tabular}


Table A.6: T6. Existential relation P between two classes A and B

\begin{tabular}{|c|c|c|c|}
\hline Goal: & \multicolumn{3}{|c|}{ T6. Existential relation $\mathrm{P}$ between two classes $\mathrm{A}$ and $\mathrm{B}$} \\
\hline Test expression: & \multicolumn{3}{|c|}{ [ClassA] subClassOf [PropertyP] some [ClassB] } \\
\hline Type: & Simple test & Related to: & Classes and properties \\
\hline \multicolumn{2}{|c|}{ Test precondition } & \multicolumn{2}{|c|}{ Test preparation } \\
\hline Class A, Class B ar & Property P exist & \multicolumn{2}{|c|}{$\begin{array}{l}\text { (Ers 1.1) Declaration of } \neg \mathrm{B} \\
\text { (Ers 1.2) Assertion } \mathrm{A}^{\prime} \sqsubseteq \mathrm{A} \\
\text { (Ers 1.3) Assertion } \mathrm{A}^{\prime}(\mathrm{a} 1) \\
\text { (Ers 1.4) Assertion } \neg \mathrm{B}(\text { nob1) } \\
\text { (Ers 1.5) Assertion } \mathrm{B}(\mathrm{b} 1)\end{array}$} \\
\hline \multicolumn{4}{|c|}{ Assertions to test the ontology behaviour } \\
\hline \multicolumn{2}{|r|}{ Axiom } & \multicolumn{2}{|r|}{ Result } \\
\hline \multicolumn{2}{|c|}{$\begin{array}{l}\text { (Ers 2.1) Assertion } \mathrm{A}^{\prime} \sqsubseteq \forall \mathrm{P} .\{\text { nob1 } \\
(\text { Ers 2.2) Assertion } \mathrm{P}(\mathrm{a} 1, \text { nob1) }\end{array}$} & \multicolumn{2}{|c|}{ Unsatisfiable class } \\
\hline \multicolumn{2}{|c|}{$\begin{array}{l}\text { (Ers 3.1) Assertion } \mathrm{A}^{\prime} \sqsubseteq \forall \mathrm{P} .\{\text { nob1, b1\} } \\
\text { (Ers 3.2) Assertion } \mathrm{P}(\mathrm{a} 1 \text {, nob1) } \\
(\text { Ers 3.3) Assertion } \mathrm{P}(\mathrm{a} 1 \text {, ind2) }\end{array}$} & \multicolumn{2}{|c|}{ Consistent ontology } \\
\hline
\end{tabular}

Table A.7: T7. Universal relation $\mathrm{P}$ between two classes A and B

\begin{tabular}{|c|c|c|c|}
\hline Goal: & \multicolumn{3}{|c|}{ T7. Universal relation $\mathrm{P}$ between two classes $\mathrm{A}$ and $\mathrm{B}$} \\
\hline Test expression: & \multicolumn{3}{|c|}{ [ClassA] subClassOf [PropertyP] only [ClassB] } \\
\hline Type: & Simple test & Related to: & Classes and properties \\
\hline \multicolumn{2}{|c|}{ Test precondition } & \multicolumn{2}{|c|}{ Test preparation } \\
\hline Class A, Class B a & Property P exist & \multicolumn{2}{|c|}{$\begin{array}{l}\text { (Urs 1.1) Declaration of } \neg \mathrm{B} \\
\text { (Urs 1.2) Assertion } \mathrm{A}^{\prime} \sqsubseteq \mathrm{A} \\
\text { (Urs 1.3) Declaration of } \mathrm{A}^{\prime}(\mathrm{a} 1) \\
\text { (Urs 1.4) Declaration of } \neg \mathrm{B}(\text { nob1) } \\
\text { (Urs 1.5) Declaration of } \mathrm{B}(\mathrm{b} 1)\end{array}$} \\
\hline \multicolumn{4}{|c|}{ Assertions to test the ontology behaviour } \\
\hline \multicolumn{2}{|r|}{ Axiom } & \multicolumn{2}{|r|}{ Result } \\
\hline \multicolumn{2}{|c|}{$\begin{array}{l}\text { (Urs 2.1) Assertion } \mathrm{A}^{\prime} \sqsubseteq \forall \mathrm{P} .\{\text { nob1 } \\
\text { (Urs 2.2) Assertion } \mathrm{P}(\mathrm{a} 1, \text { nob1) }\end{array}$} & \multicolumn{2}{|c|}{ Inconsistent ontology } \\
\hline \multicolumn{2}{|c|}{$\begin{array}{l}\text { (Urs 3.1) Assertion } \mathrm{A}^{\prime} \sqsubseteq \forall .\{\text { nob1, b1\} } \\
\text { (Urs 3.2) Assertion } \mathrm{P}(\mathrm{a} 1, \text { nob1) } \\
\text { (Urs 3.3) Assertion } \mathrm{P}(\mathrm{a} 1, \mathrm{~b} 1)\end{array}$} & \multicolumn{2}{|c|}{ Inconsistent ontology } \\
\hline \multicolumn{2}{|c|}{$\begin{array}{l}\text { (Urs 4.1) Assertion } \mathrm{A}^{\prime} \sqsubseteq \forall .\{\mathrm{b} 1\} \\
\text { (Urs 4.2) Assertion } \mathrm{P}(\mathrm{a} 1, \mathrm{~b} 1)\end{array}$} & \multicolumn{2}{|c|}{ Consistent ontology } \\
\hline
\end{tabular}


Table A.8: T8. Symmetric property P

\begin{tabular}{|c|c|c|c|}
\hline Goal: & \multicolumn{3}{|c|}{ T8. Symmetric property $\mathrm{P}$} \\
\hline Test expression: & \multicolumn{3}{|c|}{ [PropertyP] characteristic symmetricProperty } \\
\hline Type: & Simple test & Related to: & Classes and properties \\
\hline \multicolumn{2}{|c|}{ Test preconditio } & \multicolumn{2}{|c|}{ Test preparation } \\
\hline Property P exists & & \multicolumn{2}{|c|}{$\begin{array}{l}\text { (Sy 1.1) Assertion B(b1) } \\
\text { (Sy 1.2) Declaration } A^{\prime} \sqsubseteq A \\
\text { (Sy 1.3) Assertion } A^{\prime} \sqsubseteq \forall P .\{b 1\} \\
\text { (Sy 1.4) Assertion } A^{\prime}(\mathrm{a} 1) \\
\text { (Sy 1.5) Assertion } \mathrm{B}(\mathrm{b} 2)\end{array}$} \\
\hline \multicolumn{4}{|c|}{ Assertions to test the ontology behaviour } \\
\hline \multicolumn{2}{|r|}{ Axiom } & \multicolumn{2}{|r|}{ Result } \\
\hline \multicolumn{2}{|c|}{ (Sy 2) Assertion P(a1, b1) } & \multicolumn{2}{|c|}{ Consistent ontology } \\
\hline \multicolumn{2}{|c|}{ (Sy 3) Assertion $\mathrm{P}(\mathrm{b} 2, \mathrm{a} 1)$} & \multicolumn{2}{|c|}{ Inconsistent ontology } \\
\hline
\end{tabular}

Table A.9: T9. Minimum cardinality

\begin{tabular}{|c|c|c|c|}
\hline Goal: & \multicolumn{3}{|c|}{ T9. Minimum cardinality } \\
\hline Test expression: & \multicolumn{3}{|c|}{ [ClassA] subClassOf [PropertyP] min [num] [ClassB] } \\
\hline Type: & Simple test & Related to: & Classes and properties \\
\hline \multicolumn{2}{|c|}{ Test precondition } & \multicolumn{2}{|c|}{ Test preparation } \\
\hline \multicolumn{2}{|c|}{ Class A, Class B and Property P exist } & \multicolumn{2}{|c|}{ (Min 1) Declaration of $\mathrm{A}^{\prime} \sqsubseteq \mathrm{A}$} \\
\hline \multicolumn{4}{|c|}{ Assertions to test the ontology behaviour } \\
\hline \multicolumn{2}{|r|}{ Axiom } & \multicolumn{2}{|r|}{ Result } \\
\hline \multicolumn{2}{|c|}{ (Min 2) Assertion $\mathrm{A}^{\prime} \leq($ num-1)P.B } & \multicolumn{2}{|c|}{ Unsatisfiable class } \\
\hline \multicolumn{2}{|c|}{ (Min 3) Assertion $A^{\prime} \geq($ num +1$)$ P.B } & \multicolumn{2}{|c|}{ Consistent ontology } \\
\hline \multicolumn{2}{|c|}{ (Min 4) Assertion $A^{\prime} \leq$ (num)P.B } & \multicolumn{2}{|c|}{ Consistent ontology } \\
\hline \multicolumn{2}{|c|}{ (Min 5) Assertion $A^{\prime} \geq$ (num)P.B } & \multicolumn{2}{|c|}{ Consistent ontology } \\
\hline
\end{tabular}

Table A.10: T10. Maximum cardinality

\begin{tabular}{|c|c|c|c|}
\hline Goal: & \multicolumn{3}{|c|}{ T10. Maximum cardinality } \\
\hline Test expression: & \multicolumn{3}{|c|}{ [ClassA] subClassOf [PropertyP] max [num] [ClassB] } \\
\hline Type: & Simple test & Related to: & Classes and properties \\
\hline \multicolumn{2}{|c|}{ Test precondition } & \multicolumn{2}{|c|}{ Test preparation } \\
\hline \multicolumn{2}{|c|}{ Class A, Class B and Property P exist } & \multicolumn{2}{|c|}{ (Max 1) Declaration of $A^{\prime} \sqsubseteq A$} \\
\hline \multicolumn{4}{|c|}{ Assertions to test the ontology behaviour } \\
\hline \multicolumn{2}{|r|}{ Axiom } & \multicolumn{2}{|r|}{ Result } \\
\hline \multicolumn{2}{|c|}{ (Max 2) Assertion $A^{\prime} \leq($ num-1)P.B } & \multicolumn{2}{|c|}{ Consistent ontology } \\
\hline \multicolumn{2}{|c|}{ (Max 3) Assertion $A^{\prime} \geq($ num +1$)$ P.B } & \multicolumn{2}{|c|}{ Unsatisfiable class } \\
\hline \multicolumn{2}{|c|}{ (Max 4) Assertion $A^{\prime} \leq$ (num)P.B } & \multicolumn{2}{|c|}{ Consistent ontology } \\
\hline \multicolumn{2}{|c|}{ (Max 5) Assertion $A^{\prime} \geq($ num)P.B } & \multicolumn{2}{|c|}{ Consistent ontology } \\
\hline
\end{tabular}


Table A.11: T11. Exact cardinality

\begin{tabular}{|c|c|c|c|}
\hline Goal: & \multicolumn{3}{|l|}{ T11. Exact cardinality } \\
\hline Test expression: & \multicolumn{3}{|c|}{ [ClassA] subClassOf [PropertyP] exactly [num] [ClassB] } \\
\hline Type: & Simple test & Related to: & Classes and properties \\
\hline \multicolumn{2}{|c|}{ Test precondition } & \multicolumn{2}{|c|}{ Test preparation } \\
\hline \multicolumn{2}{|c|}{ Class A, Class B and Property P exist } & \multicolumn{2}{|c|}{ (Ex 1) Declaration of $A^{\prime} \sqsubseteq A$} \\
\hline \multicolumn{4}{|c|}{ Assertions to test the ontology behaviour } \\
\hline \multicolumn{2}{|r|}{ Axiom } & \multicolumn{2}{|r|}{ Result } \\
\hline \multicolumn{2}{|c|}{ (Ex 2) Assertion $A^{\prime} \leq($ num-1)P.B } & \multicolumn{2}{|c|}{ Unsatisfiable class } \\
\hline \multicolumn{2}{|c|}{ (Ex 3) Assertion $A^{\prime} \geq($ num +1$)$ P.B } & \multicolumn{2}{|c|}{ Unsatisfiable class } \\
\hline \multicolumn{2}{|c|}{$\left(\right.$ Ex 4) Assertion $A^{\prime} \leq$ (num)P.B } & \multicolumn{2}{|c|}{ Consistent ontology } \\
\hline \multicolumn{2}{|c|}{ (Ex 5) Assertion $A^{\prime} \geq($ num)P.B } & \multicolumn{2}{|c|}{ Consistent ontology } \\
\hline
\end{tabular}

Table A.12: T12. Universal relation $\mathrm{P}$ between the union of two classes $\mathrm{A}$ and $\mathrm{B}$

\begin{tabular}{|c|c|c|c|}
\hline Goal: & \multicolumn{3}{|c|}{ T12. Universal relation $\mathrm{P}$ between the union of two classes $\mathrm{A}$ and $\mathrm{B}$} \\
\hline Test expression: & \multicolumn{3}{|c|}{ [ClassA] subClassOf [PropertyP] only [ClassB] or [ClassC] } \\
\hline Type: & Simple test & Related to: & Classes and properties \\
\hline \multicolumn{2}{|c|}{ Test precondition } & \multicolumn{2}{|c|}{ Test preparation } \\
\hline \multicolumn{2}{|c|}{ Class A, Class B and Property P exist } & \multicolumn{2}{|c|}{$\begin{array}{l}\text { (U 1.1) Declaration of } \neg \mathrm{B} \\
\text { (U 1.2) Assertion A' } \sqsubseteq \mathrm{A} \\
\text { (U 1.3) Declaration of } \neg \mathrm{C}\end{array}$} \\
\hline \multicolumn{4}{|c|}{ Assertions to test the ontology behaviour } \\
\hline \multicolumn{2}{|r|}{ Axiom } & \multicolumn{2}{|r|}{ Result } \\
\hline \multicolumn{2}{|c|}{ (U 2) Assertion A' $\sqsubseteq \exists$ P.B } & \multicolumn{2}{|c|}{ Consistent ontology } \\
\hline \multicolumn{2}{|c|}{ (U 3) Assertion A' $\sqsubseteq \exists$ P.C } & \multicolumn{2}{|c|}{ Consistent ontology } \\
\hline \multicolumn{2}{|c|}{ (U 4) Assertion $\mathrm{A}^{\prime} \sqsubseteq \exists \mathrm{P} . \neg \mathrm{B} \sqcap \neg \mathrm{C}$} & \multicolumn{2}{|c|}{ Inconsistent ontology } \\
\hline \multicolumn{2}{|c|}{ (U 5) Assertion $\mathrm{A}^{\prime} \sqsubseteq \exists \mathrm{P} . \mathrm{C} \sqcap \neg \mathrm{B}$} & \multicolumn{2}{|c|}{ Consistent ontology } \\
\hline \multicolumn{2}{|c|}{ (U 6) Assertion A' $\sqsubseteq \exists \mathrm{P} . \neg \mathrm{C} \sqcap \mathrm{B}$} & \multicolumn{2}{|c|}{ Consistent ontology } \\
\hline \multicolumn{2}{|c|}{ (U 7) Assertion $\mathrm{A}^{\prime} \sqsubseteq \exists \mathrm{P} . \mathrm{C} \sqcap \mathrm{B}$} & \multicolumn{2}{|c|}{ Consistent ontology } \\
\hline
\end{tabular}


Table A.13: T13. Universal relation $\mathrm{P}$ between the intersection of two classes $\mathrm{A}$ and $\mathrm{B}$

\begin{tabular}{|c|c|c|c|}
\hline Goal: & \multicolumn{3}{|c|}{ T13. Universal relation $\mathrm{P}$ between the intersection of two classes $\mathrm{A}$ and $\mathrm{B}$} \\
\hline Test expression: & \multicolumn{3}{|c|}{ [ClassA] subClassOf [PropertyP] only [ClassB] and [ClassC] } \\
\hline Type: & Simple test & Related to: & Classes and properties \\
\hline \multicolumn{2}{|c|}{ Test precondition } & \multicolumn{2}{|c|}{ Test preparation } \\
\hline \multicolumn{2}{|c|}{ Class A, Class B and Property P exist } & \multicolumn{2}{|c|}{$\begin{array}{l}\text { (Isc 1.1) Declaration of } \neg \mathrm{B} \\
\text { (Isc 1.2) Assertion } \mathrm{A}^{\prime} \sqsubseteq \mathrm{A} \\
\text { (Isc 1.3) Declaration of } \neg \mathrm{C}\end{array}$} \\
\hline \multicolumn{4}{|c|}{ Assertions to test the ontology behaviour } \\
\hline \multicolumn{2}{|r|}{ Axiom } & \multicolumn{2}{|r|}{ Result } \\
\hline \multicolumn{2}{|c|}{ (Isc 2) Assertion $A^{\prime} \sqsubseteq \exists$ P.B } & \multicolumn{2}{|c|}{ Consistent ontology } \\
\hline \multicolumn{2}{|c|}{ (Isc 3) Assertion A' $\sqsubseteq \exists$ P.C } & \multicolumn{2}{|c|}{ Consistent ontology } \\
\hline \multicolumn{2}{|c|}{ (Isc 4) Assertion $\mathrm{A}^{\prime} \sqsubseteq \exists \mathrm{P} . \neg \mathrm{C} \sqcap \neg \mathrm{B}$} & \multicolumn{2}{|c|}{ Unsatisfiable class } \\
\hline \multicolumn{2}{|c|}{ (Isc 5) Assertion $\mathrm{A}^{\prime} \sqsubseteq \exists \mathrm{P} . \mathrm{C} \sqcap \neg \mathrm{B}$} & \multicolumn{2}{|c|}{ Unsatisfiable class } \\
\hline \multicolumn{2}{|c|}{ (Isc 6) Assertion $\mathrm{A}^{\prime} \sqsubseteq \exists \mathrm{P} . \neg \mathrm{C} \sqcap \mathrm{B}$} & \multicolumn{2}{|c|}{ Unsatisfiable class } \\
\hline \multicolumn{2}{|c|}{ (Isc 7) Assertion $\mathrm{A}^{\prime} \sqsubseteq \exists \mathrm{P} . \mathrm{C} \sqcap \mathrm{B}$} & \multicolumn{2}{|c|}{ Consistent ontology } \\
\hline
\end{tabular}

Table A.14: T14. Individual I exists

\begin{tabular}{|c|c|c|c|}
\hline Goal: & \multicolumn{3}{|l|}{ T14. Individual I exists } \\
\hline Test expression: & \multicolumn{3}{|l|}{ [IndividualI] type [ClassA] } \\
\hline Type: & Simple test & Related to: & Individuals and Classes \\
\hline \multicolumn{2}{|c|}{ Test precondition } & \multicolumn{2}{|c|}{ Test preparation } \\
\hline \multicolumn{2}{|c|}{ Class A and Individual I exist } & \multicolumn{2}{|c|}{ (I 1)Declaration of $\neg \mathrm{A}$} \\
\hline \multicolumn{4}{|c|}{ Assertions to test the ontology behaviour } \\
\hline \multicolumn{2}{|r|}{ Axiom } & \multicolumn{2}{|r|}{ Result } \\
\hline \multicolumn{2}{|c|}{ (I 2) Assertion $\neg \mathrm{A}$ (a1) } & \multicolumn{2}{|c|}{ Inconsistent ontology } \\
\hline
\end{tabular}

Table A.15: T15. Property P has domain Class A

\begin{tabular}{|c|c|c|c|}
\hline Goal: & \multicolumn{3}{|c|}{ T15. Property $\mathrm{P}$ has domain Class A } \\
\hline Test expression: & \multicolumn{3}{|c|}{ [PropertyP] domain [ClassA] } \\
\hline Type: & Simple test & Related to: & Classes and Properties \\
\hline \multicolumn{2}{|c|}{ Test preconditio } & \multicolumn{2}{|c|}{ Test preparation } \\
\hline \multicolumn{2}{|c|}{ Class A and Property P exist } & \multicolumn{2}{|c|}{$\begin{array}{l}\text { (R 1.1) Declaration of } \neg \mathrm{A} \\
\text { ( } \mathrm{R} \text { 1.2) Assertion } \neg \mathrm{A}(\text { noa } 1) \\
\text { (R 1.3) Assertion Thing(b1) }\end{array}$} \\
\hline \multicolumn{4}{|c|}{ Assertions to test the ontology behaviour } \\
\hline \multicolumn{2}{|r|}{ Axiom } & \multicolumn{2}{|r|}{ Result } \\
\hline \multicolumn{2}{|c|}{ (R 2) Assertion P(noa1,b1) } & \multicolumn{2}{|c|}{ Inconsistent ontology } \\
\hline \multicolumn{2}{|c|}{ (R 3) Assertion P(a1,b1) } & \multicolumn{2}{|c|}{ Consistent ontology } \\
\hline
\end{tabular}


Table A.16: T16. Property $\mathrm{P}$ has range Class A

\begin{tabular}{|c|c|c|c|}
\hline Goal: & \multicolumn{3}{|c|}{ T16. Property $\mathrm{P}$ has range Class $\mathrm{A}$} \\
\hline Test expression: & \multicolumn{3}{|c|}{ [PropertyP] range [ClassA] } \\
\hline Type: & Simple test & Related to: & Classes and Properties \\
\hline \multicolumn{2}{|c|}{ Test preconditio } & \multicolumn{2}{|c|}{ Test preparation } \\
\hline \multicolumn{2}{|c|}{ Class A and Property P exist } & \multicolumn{2}{|c|}{$\begin{array}{l}\text { (R 1.1) Declaration of } \neg \mathrm{A} \\
\text { (R 1.2) Assertion } \neg \mathrm{A}(\text { noa1) } \\
\text { (R 1.3) Assertion Thing(b1) }\end{array}$} \\
\hline \multicolumn{4}{|c|}{ Assertions to test the ontology behaviour } \\
\hline \multicolumn{2}{|r|}{ Axiom } & \multicolumn{2}{|r|}{ Result } \\
\hline \multicolumn{2}{|c|}{ (R 2) Assertion P(b1, noa1) } & \multicolumn{2}{|c|}{ Inconsistent ontology } \\
\hline \multicolumn{2}{|c|}{ (R 3) Assertion P(b1, a1) } & \multicolumn{2}{|c|}{ Consistent ontology } \\
\hline
\end{tabular}

Table A.17: T17. Multiple inheritance of a class

\begin{tabular}{|c|c|c|c|}
\hline Goal: & \multicolumn{3}{|c|}{ T17. Multiple inheritance of a class $\mathrm{A}$ regarding classes $\mathrm{B}$ and $\mathrm{C}$} \\
\hline Test expression: & \multicolumn{3}{|c|}{ [ClassA] subClassOf [ClassB] and [ClassC] } \\
\hline Type: & Composed test & Related to: & Classes \\
\hline \multicolumn{2}{|c|}{ Test precondition } & \multicolumn{2}{|c|}{ Test preparation } \\
\hline \multicolumn{2}{|c|}{ Class A, Class B and Class C exist } & \multicolumn{2}{|c|}{$\begin{array}{l}\text { (MS 1.1) Declaration of } \neg \mathrm{A} \\
\text { (MS 1.2) Declaration of } \neg \mathrm{B} \\
\text { (MS 1.3) Declaration of } \neg \mathrm{C}\end{array}$} \\
\hline \multicolumn{4}{|c|}{ Assertions to test the ontology behaviour } \\
\hline \multicolumn{2}{|r|}{ Axiom } & \multicolumn{2}{|c|}{ Result } \\
\hline \multicolumn{2}{|c|}{$(\mathrm{MS} 2) \mathrm{A}^{\prime} \sqsubseteq \neg \mathrm{A} \sqcap \mathrm{B}$} & \multicolumn{2}{|c|}{ Consistent ontology } \\
\hline \multicolumn{2}{|c|}{$(\mathrm{MS} 3) \mathrm{A}^{\prime} \sqsubseteq \mathrm{A} \sqcap \neg \mathrm{B}$} & \multicolumn{2}{|c|}{ Unsatisfiable class } \\
\hline \multicolumn{2}{|l|}{$(\mathrm{MS} 4) \mathrm{A}^{\prime} \sqsubseteq \mathrm{A} \sqcap \mathrm{B}$} & \multicolumn{2}{|c|}{ Consistent ontology } \\
\hline \multicolumn{2}{|c|}{$(\mathrm{MS} 5) \mathrm{A}^{\prime} \sqsubseteq \neg \mathrm{A} \sqcap \mathrm{C}$} & \multicolumn{2}{|c|}{ Consistent ontology } \\
\hline \multicolumn{2}{|c|}{$(\mathrm{MS} 6) \mathrm{A}^{\prime} \sqsubseteq \mathrm{A} \sqcap \neg \mathrm{C}$} & \multicolumn{2}{|c|}{ Unsatisfiable class } \\
\hline \multicolumn{2}{|l|}{$(\mathrm{MS} 7) \mathrm{A}^{\prime} \sqsubseteq \mathrm{A} \sqcap \mathrm{C}$} & \multicolumn{2}{|c|}{ Consistent ontology } \\
\hline
\end{tabular}


Table A.18: T18. There is a subsumption relation between two classes and an existential restriction

\begin{tabular}{|c|c|c|c|}
\hline Goal: & \multicolumn{3}{|c|}{$\begin{array}{l}\text { T18. Subsumption relation between two classes } \mathrm{A} \text { and } \mathrm{B} \text {, and an existential } \\
\text { relation } \mathrm{P} \text { between } \mathrm{B} \text { and } \mathrm{C}\end{array}$} \\
\hline Test expression: & \multicolumn{3}{|c|}{ [ClassA] subClassOf [ClassB] that [PropertyP] some [ClassC] } \\
\hline Type: & Composed test & Related to: & Classes \\
\hline \multicolumn{2}{|c|}{ Test precondition } & \multicolumn{2}{|c|}{ Test preparation } \\
\hline \multicolumn{2}{|c|}{ Class A and Class B exist } & \multicolumn{2}{|c|}{$\begin{array}{l}\text { (SErs 1.1) Declaration of } \neg \mathrm{A} \\
\text { (SErs 1.2) Declaration of } \neg \mathrm{B} \\
\text { (SErs 1.3) Assertion A' } \sqsubseteq \mathrm{A} \\
\text { (SErs 1.4) Assertion } \mathrm{A}^{\prime}(\mathrm{a} 1) \\
\text { (SErs 1.6) Declaration of } \neg \mathrm{C} \\
\text { (SErs 1.5) Assertion } \neg \mathrm{C}(\text { noc } 1) \\
\text { (SErs 1.6) Declaration of } \mathrm{C}(\text { noc } 1)\end{array}$} \\
\hline \multicolumn{4}{|c|}{ Assertions to test the ontology behaviour } \\
\hline \multicolumn{2}{|r|}{ Axiom } & \multicolumn{2}{|r|}{ Result } \\
\hline \multicolumn{2}{|c|}{ (SErs 2) $\mathrm{A}^{\prime} \sqsubseteq \neg \mathrm{A} \sqcap \mathrm{B}$} & \multicolumn{2}{|c|}{ Consistent ontology } \\
\hline \multicolumn{2}{|c|}{ (SErs 3) $\mathrm{A}^{\prime} \sqsubseteq \mathrm{A} \sqcap \neg \mathrm{B}$} & \multicolumn{2}{|c|}{ Unsatisfiable class } \\
\hline \multicolumn{2}{|c|}{ (SErs 4) $\mathrm{A}^{\prime} \sqsubseteq \mathrm{A} \sqcap \neg \mathrm{B}$} & \multicolumn{2}{|c|}{ Consistent ontology } \\
\hline \multicolumn{2}{|c|}{$\begin{array}{l}\text { (SErs 4.1) Assertion } \mathrm{A}^{\prime} \sqsubseteq \forall \mathrm{P} .\{\text { noc } 1\} \\
(\text { SErs } 4.2) \text { Assertion } \mathrm{P}(\mathrm{a} 1, \text { noc } 1)\end{array}$} & \multicolumn{2}{|c|}{ Unsatisfiable class } \\
\hline \multicolumn{2}{|c|}{$\begin{array}{l}\text { (SErs 5.1) Assertion } A^{\prime} \sqsubseteq \forall P .\{\text { noc1, } \mathrm{c} 1\} \\
\text { (SErs 5.2) Assertion } \mathrm{P}(\mathrm{a} 1, \text { noc1) } \\
(\text { SErs 5.3) Assertion } \mathrm{P}(\mathrm{a} 1, \mathrm{c} 1)\end{array}$} & \multicolumn{2}{|c|}{ Consistent ontology } \\
\hline
\end{tabular}


Table A.19: T19. Minimum cardinality and existential relation

\begin{tabular}{|c|c|c|c|}
\hline Goal: & \multicolumn{3}{|c|}{$\begin{array}{l}\text { T19. Minimum cardinality between classes } \mathrm{A} \text { and } \mathrm{B} \text {, and existential relation } \\
\mathrm{P} \text { between classes B and } \mathrm{C}\end{array}$} \\
\hline Test expression: & \multicolumn{3}{|c|}{$\begin{array}{l}\text { [ClassA] subClassOf [PropertyP] min [ClassB] and [ClassB] } \\
\text { subClassOf [PropertyD] some [ClassC] }\end{array}$} \\
\hline Type: & Composed test & Related to: & Classes and properties \\
\hline \multicolumn{2}{|r|}{ Test precondition } & \multicolumn{2}{|c|}{ Test preparation } \\
\hline Class A, Class B, C & ass C, Property P and Property D exist & $\begin{array}{l}\text { (MinErs 1.1) } \\
\text { (MinErs 1.2) } \\
\text { (MinErs 1.3) } \\
\text { (MinErs 1.4) } \\
\text { (MinErs 1.5) } \\
\text { (MinErs 1.6) }\end{array}$ & $\begin{array}{l}\text { eclaration of } A^{\prime} \sqsubseteq A \\
\text { ssertion } B^{\prime}(b 1) \\
\text { ssertion } \neg \mathrm{B}(\text { nob1) } \\
\text { eclaration } \neg \mathrm{C} \\
\text { ssertion } \neg \mathrm{C}(\text { noc } 1) \\
\text { ssertion } \mathrm{C}(\mathrm{c} 1)\end{array}$ \\
\hline \multicolumn{4}{|c|}{ Assertions to test the ontology behaviour } \\
\hline & Axiom & \multirow{2}{*}{\multicolumn{2}{|c|}{$\begin{array}{r}\text { Result } \\
\text { Unsatisfiable class }\end{array}$}} \\
\hline (MinErs 2) Assertio & $\mathrm{A}^{\prime} \leq($ num-1)P.B & & \\
\hline (MinErs 3) Assertio & $A^{\prime} \geq($ num +1$)$ P.B & \multicolumn{2}{|c|}{ Consistent ontology } \\
\hline (MinErs 4) Assertio & $\mathrm{A}^{\prime} \leq$ (num)P.B & \multicolumn{2}{|c|}{ Consistent ontology } \\
\hline \multicolumn{2}{|c|}{ (MinErs 5) Assertion $\mathrm{A}^{\prime} \geq$ (num)P.B } & \multicolumn{2}{|c|}{ Consistent ontology } \\
\hline \multicolumn{2}{|c|}{$\begin{array}{l}\text { (MinErs 6.1) Assertion B' } \sqsubseteq \forall \mathrm{D} .\{\text { noc } 1\} \\
\text { (MinErs 6.2) Assertion D(b1, noc1) }\end{array}$} & \multicolumn{2}{|c|}{ Unsatisfiable class } \\
\hline \multicolumn{2}{|c|}{$\begin{array}{l}\text { (MinErs 7.1) Assertion B' } \sqsubseteq \forall D .\{\text { noc } 1, \mathrm{c} 1\} \\
(\text { MinErs 7.2) Assertion } \mathrm{D}(\mathrm{b} 1, \operatorname{noc} 1) \\
(\text { MinErs 7.3) Assertion } \mathrm{D}(\mathrm{b} 1, \mathrm{c} 1)\end{array}$} & \multicolumn{2}{|c|}{ Consistent ontology } \\
\hline
\end{tabular}


Table A.20: T20. Minimum cardinality and universal restriction

\begin{tabular}{|c|c|c|c|}
\hline Goal: & \multicolumn{3}{|c|}{$\begin{array}{l}\text { T20. Minimum cardinality between classes } \mathrm{A} \text { and } \mathrm{B} \text { and universal relation } \\
\mathrm{P} \text { between classes } \mathrm{B} \text { and } \mathrm{C}\end{array}$} \\
\hline Test expression: & \multicolumn{3}{|c|}{$\begin{array}{l}\text { [ClassA] subClassOf [PropertyP] min [ClassB] and [ClassB] } \\
\text { subClassOf [PropertyD] only [ClassC] }\end{array}$} \\
\hline Type: & Composed test & Related to: & Classes and properties \\
\hline \multicolumn{2}{|r|}{ Test precondition } & \multicolumn{2}{|c|}{ Test preparation } \\
\hline Class A, Class B, & ass, Property P and Property D exist & $\begin{array}{l}\text { (MinUrs } 1.1) \\
(\text { MinUrs } 1.2) \\
(\text { MinUrs } 1.3) \\
\text { (MinUrs } 1.4) \mathrm{I} \\
\text { (MinUrs 1.5) }\end{array}$ & $\begin{array}{l}\text { Declaration of } \mathrm{A}^{\prime} \sqsubseteq \mathrm{A} \\
\text { Declaration of } \neg \mathrm{B} \\
\text { Declaration of } \mathrm{B}^{\prime}(\mathrm{b} 1) \\
\text { eclaration of } \neg \mathrm{C}(\mathrm{noc} 1) \\
\text { eclaration of } \mathrm{C}(\mathrm{c} 1)\end{array}$ \\
\hline \multicolumn{4}{|c|}{ Assertions to test the ontology behaviour } \\
\hline & Axiom & & Result \\
\hline (MinUrs 2) Asserti & $\mathrm{A}^{\prime} \leq$ (num-1)P.B & Unsatisfiable & lass \\
\hline (MinUrs 3) Asserti & $A^{\prime} \geq($ num +1$)$ P.B & Consistent on & ology \\
\hline (MinUrs 4) Asserti & $\mathrm{A}^{\prime} \leq$ (num)P.B & Consistent on & ology \\
\hline (MinUrs 5) Asserti & $\mathrm{A}^{\prime} \geq$ (num)P.B & Consistent on & ology \\
\hline $\begin{array}{l}\text { (MinUrs 6.1) Asser } \\
\text { (MinUrs 6.2) Asser }\end{array}$ & $\begin{array}{l}\text { on } \mathrm{B}^{\prime} \sqsubseteq \forall \mathrm{D} \cdot\{\text { noc } 1\} \\
\text { on } \mathrm{D}(\mathrm{b} 1, \text { noc } 1)\end{array}$ & Unsatisfiable & lass \\
\hline $\begin{array}{l}\text { (MinUrs 7.1) Asser } \\
\text { (MinUrs 7.2) Asser } \\
\text { (MinUrs 7.3) Asser }\end{array}$ & $\begin{array}{l}\text { on } \mathrm{B}^{\prime} \sqsubseteq \forall \mathrm{D} .\{\text { noc } 1, \mathrm{c} 1\} \\
\text { on } \mathrm{D}(\mathrm{b} 1, \text { noc } 1) \\
\text { on } \mathrm{D}(\mathrm{b} 1, \mathrm{c} 1)\end{array}$ & Inconsistent o & tology \\
\hline $\begin{array}{l}\text { MinUrs 8.1) Asser } \\
\text { (MinUrs 8.2) Asser }\end{array}$ & $\begin{array}{l}\text { on } \mathrm{A}^{\prime} \sqsubseteq \forall \mathrm{D} \cdot\{\mathrm{b} 1\} \\
\text { on } \mathrm{D}(\mathrm{a} 1, \mathrm{~b} 1)\end{array}$ & Consistent on & ology \\
\hline
\end{tabular}


Table A.21: T21. Subsumption relation between A and B, subsumption relation between $\mathrm{A}$ and $\mathrm{C}$, and disjointness between $\mathrm{B}$ and $\mathrm{C}$

\begin{tabular}{|c|c|c|c|}
\hline Goal: & \multicolumn{3}{|c|}{$\begin{array}{l}\text { T21. Subsumption relation between } \mathrm{A} \text { and } \mathrm{B} \text {, subsumption relation } \\
\text { between } \mathrm{C} \text { and } \mathrm{B} \text {, and disjointness between } \mathrm{A} \text { and } \mathrm{C}\end{array}$} \\
\hline Test expression: & \multicolumn{3}{|c|}{$\begin{array}{l}\text { [ClassA] subClassOf [ClassB] and [ClassC] subClassOf [ClassB] that } \\
\text { disjointWith [ClassA] }\end{array}$} \\
\hline Type: & Composed test & Related to: & Classes \\
\hline \multicolumn{2}{|c|}{ Test precondition } & \multicolumn{2}{|c|}{ Test preparation } \\
\hline \multicolumn{2}{|c|}{ Class A, Class B and Class C exist } & \multicolumn{2}{|c|}{$\begin{array}{l}\text { (SD 1.1) Declaration of } \neg \mathrm{A} \\
\text { (SD 1.2) Declaration of } \neg \mathrm{B} \\
\text { (SD 1.3) Declaration of } \neg \mathrm{C}\end{array}$} \\
\hline \multicolumn{4}{|c|}{ Assertions to test the ontology behaviour } \\
\hline \multicolumn{2}{|r|}{ Axiom } & \multicolumn{2}{|c|}{ Result } \\
\hline \multicolumn{2}{|l|}{$(\mathrm{SD} 2) \mathrm{A}^{\prime} \sqsubseteq \neg \mathrm{A} \sqcap \mathrm{B}$} & \multicolumn{2}{|c|}{ Consistent ontology } \\
\hline \multicolumn{2}{|l|}{$(\mathrm{SD} 3) \mathrm{A}^{\prime} \sqsubseteq \mathrm{A} \sqcap \neg \mathrm{B}$} & \multicolumn{2}{|c|}{ Unsatisfiable class } \\
\hline \multicolumn{2}{|l|}{$(\mathrm{SD} 4) \mathrm{A}^{\prime} \sqsubseteq \mathrm{A} \sqcap \mathrm{B}$} & \multicolumn{2}{|c|}{ Consistent ontology } \\
\hline \multicolumn{2}{|l|}{$(\mathrm{SD} 5) \mathrm{C}^{\prime} \sqsubseteq \neg \mathrm{C} \sqcap \mathrm{B}$} & \multicolumn{2}{|c|}{ Consistent ontology } \\
\hline \multicolumn{2}{|l|}{$(\mathrm{SD} 6) \mathrm{C}^{\prime} \sqsubseteq \mathrm{C} \sqcap \neg \mathrm{B}$} & \multicolumn{2}{|c|}{ Unsatisfiable class } \\
\hline \multicolumn{2}{|l|}{$(\mathrm{SD} 7) \mathrm{C}^{\prime} \sqsubseteq \mathrm{C} \sqcap \mathrm{B}$} & \multicolumn{2}{|c|}{ Consistent ontology } \\
\hline \multicolumn{2}{|c|}{$(\mathrm{SD} 5) \mathrm{A}^{\prime} \sqsubseteq \neg \mathrm{A} \sqcap \mathrm{C}$} & \multicolumn{2}{|c|}{ Consistent ontology } \\
\hline \multicolumn{2}{|c|}{$(\mathrm{SD} 6) \mathrm{A}^{\prime} \sqsubseteq \mathrm{A} \sqcap \neg \mathrm{C}$} & \multicolumn{2}{|c|}{ Consistent ontology } \\
\hline \multicolumn{2}{|l|}{$(\mathrm{SD} 7) \mathrm{A}^{\prime} \sqsubseteq \mathrm{A} \sqcap \mathrm{C}$} & \multicolumn{2}{|c|}{ Unsatisfiable class } \\
\hline
\end{tabular}


Table A.22: T22. Participation ODP between classes A and B

\begin{tabular}{|c|c|c|c|}
\hline Goal: & \multicolumn{3}{|c|}{ T22. Participation ODP between classes A and B } \\
\hline Test expression: & \multicolumn{3}{|c|}{ [ClassA] subClassOf [participates] some [ClassB] } \\
\hline Type: & ODP-based test & Related to: & Classes and properties \\
\hline \multicolumn{2}{|c|}{ Test precondition } & \multicolumn{2}{|c|}{ Test preparation } \\
\hline \multicolumn{2}{|c|}{$\begin{array}{l}\text { Class A, Class B, Property participates and Property } \\
\text { isParticipantIn exist }\end{array}$} & $\begin{array}{l}\text { (ODPPa 1.1) } \\
(\text { ODPPa 1.2) } \\
(\text { ODPPa 1.3) } \\
(\text { ODPPa 1.4) } \\
(\text { ODPPa 1.5) } \\
(\text { ODPPa 1.6) } \\
(\text { ODPPa 1.7) } \\
(\text { ODPPa } 1.8)\end{array}$ & $\begin{array}{l}\text { Declaration of } \neg \mathrm{B} \\
\text { Assertion } \mathrm{A}^{\prime} \sqsubseteq \mathrm{A} \\
\text { Assertion } \mathrm{A}^{\prime}(\mathrm{a} 1) \\
\text { Assertion } \neg \mathrm{B}(\text { nob1) } \\
\text { Declaration of } \neg \mathrm{A} \\
\text { Assertion } \mathrm{B}^{\prime} \sqsubseteq \mathrm{B} \\
\text { Assertion } \mathrm{B}^{\prime}(\mathrm{b} 1) \\
\text { Assertion } \neg \mathrm{A}(\text { noa1) }\end{array}$ \\
\hline \multicolumn{4}{|c|}{ Assertions to test the ontology behaviour } \\
\hline \multicolumn{2}{|r|}{ Axiom } & \multicolumn{2}{|r|}{ Result } \\
\hline \multicolumn{2}{|c|}{$\begin{array}{l}\text { (ODPPa 2.1) Assertion A' } \sqsubseteq \forall \text { participates. }\{\text { nob1\} } \\
(\text { ODPPa 2.2) Assertion participates }(\mathrm{a} 1, \text { nob1) }\end{array}$} & \multicolumn{2}{|c|}{ Unsatisfiable class } \\
\hline \multicolumn{2}{|c|}{$\begin{array}{l}\text { (ODPPa } 3.1) \text { Assertion A' } \sqsubseteq \forall \text { participates. }\{\text { nob1, b1\} } \\
\text { (ODPPa 3.2) Assertion participates }(\mathrm{a} 1, \text { nob1) } \\
\text { (ODPPa 3.3) Assertion participates }(\mathrm{a} 1, \mathrm{~b} 1)\end{array}$} & \multicolumn{2}{|c|}{ Consistent ontology } \\
\hline \multicolumn{2}{|c|}{$\begin{array}{l}\text { (ODPPa 4.1) Assertion B' } \sqsubseteq \forall i s P a r t i c i p a n t I n .\{\text { noa1\} } \\
(\text { ODPPa 4.2) Assertion isParticipantIn }(\mathrm{b} 1, \text { noa1) }\end{array}$} & \multicolumn{2}{|c|}{ Unsatisfiable class } \\
\hline \multicolumn{2}{|c|}{$\begin{array}{l}\text { (ODPPa 5.1) Assertion B' } \sqsubseteq \forall P .\{\text { noa1, a1\} } \\
\text { (ODPPa 5.2) Assertion isParticipantIn(b1, noa1) } \\
\text { (ODPPa 5.3) Assertion isParticipantIn(b1, a1) }\end{array}$} & \multicolumn{2}{|c|}{ Consistent ontology } \\
\hline
\end{tabular}


Table A.23: T23. Co-participation ODP

\begin{tabular}{|c|c|c|c|}
\hline Goal: & \multicolumn{3}{|l|}{ T23. CoParticipation ODP } \\
\hline Test expression: & \multicolumn{3}{|c|}{ [ClassA] and [ClassB] subClassOf [coparticipatesWith] some [ClassC] } \\
\hline Type: & ODP-based test & Related to: & Classes and properties \\
\hline \multicolumn{2}{|c|}{ Test precondition } & \multicolumn{2}{|r|}{ Test preparation } \\
\hline \multicolumn{2}{|c|}{$\begin{array}{l}\text { Class A, Class B, Property participates, Property } \\
\text { isParticipantIn and Property coparticipatesWith exist }\end{array}$} & $\begin{array}{l}\text { (ODPCPa } 1.1 \\
\text { (ODPCPa } 1.2 \\
\text { (ODPCPa } 1.3 \\
\text { (ODPCPa } 1.4 \\
\text { (ODPCPa) A } \\
\text { (ODPCPa } 1.5 \\
\text { (ODPCPa } 1.6 \\
\text { (ODPCPa } 1.7 \\
\text { (ODPCPa } 1.8 \\
\text { (ODPCPa } 1.9 \\
\text { (ODPCPa } 1.1\end{array}$ & $\begin{array}{l}\text { Declaration of } \neg \mathrm{C} \\
\text { Assertion A' } \mathrm{A} \\
\text { Assertion A'(a1) } \\
\text { Declaration of } \neg \mathrm{A} \\
\text { sertion } \mathrm{C}^{\prime} \sqsubseteq \mathrm{C} \\
\text { Assertion } \mathrm{C}^{\prime}(\mathrm{c} 1) \\
\text { Assertion } \neg \mathrm{C}(\text { noc1) } \\
\text { Assertion } \neg \mathrm{A}(\text { noa } 1) \\
\text { Assertion } \mathrm{B}(\mathrm{b} 1) \\
\text { Assertion A' } \sqsubseteq \forall \text { coparticipates With. }\{\mathrm{b} 1\} \\
\text { Assertion } \mathrm{B}(\mathrm{b} 2)\end{array}$ \\
\hline \multicolumn{2}{|c|}{$\begin{array}{l}\text { (ODPCPa 2.1) Assertion A' } \sqsubseteq \forall \text { participates. }\{\text { noc1\} } \\
\text { (ODPCPa 2.2) Assertion participates (a1, noc1) }\end{array}$} & \multicolumn{2}{|c|}{ Unsatisfiable class } \\
\hline \multicolumn{2}{|c|}{$\begin{array}{l}\text { (ODPCPa 3.1) Assertion A' } 5 \forall \text { participates. }\{\text { noc1, c1 }\} \\
\text { (ODPCPa) Assertion participates }(\mathrm{a} 1, \text { noc1) } \\
\text { (ODPCPa 3.3) Assertion participates }(\mathrm{a} 1, \mathrm{c} 1)\end{array}$} & \multicolumn{2}{|c|}{ Consistent ontology } \\
\hline \multicolumn{2}{|c|}{$\begin{array}{l}\text { (ODPCPa 4.1) Assertion B' } \sqsubseteq \forall \text { isParticipantIn. }\{\text { noa1\} } \\
\text { (ODPCPa 4.2) Assertion isParticipantIn(c1, noa } 1)\end{array}$} & \multicolumn{2}{|c|}{ Unsatisfiable class } \\
\hline \multicolumn{2}{|c|}{$\begin{array}{l}\text { (ODPCPa) Assertion B' } \sqsubseteq \forall P .\{\text { noa1, a1 }\} \\
\text { (ODPCPa 5.2) Assertion isParticipantIn }(\mathrm{c} 1, \text { noa1) } \\
\text { (ODPCPa 5.3) Assertion isParticipantIn }(\mathrm{c} 1, \mathrm{a} 1)\end{array}$} & \multicolumn{2}{|c|}{ Consistent ontology } \\
\hline \multicolumn{2}{|c|}{ (ODPCPa 6.1) Assertion coparticipates With (a1, b1) } & \multicolumn{2}{|c|}{ Consistent ontology } \\
\hline \multicolumn{2}{|c|}{ (ODPCPa 6.2) Assertion coparticipatesWith(b2, a1) } & \multicolumn{2}{|c|}{ Inconsistent ontology } \\
\hline
\end{tabular}


Table A.24: T24. PartOf ODP between classes A and B

\begin{tabular}{|c|c|c|c|}
\hline Goal: & \multicolumn{3}{|c|}{ T24. PartOf ODP between classes A and B } \\
\hline Test expression: & \multicolumn{3}{|c|}{ [ClassA] subClassOf [isPartOf] some [ClassB] } \\
\hline Type: & ODP-based test & Related to: & Classes and properties \\
\hline \multicolumn{2}{|c|}{ Test precondition } & \multicolumn{2}{|c|}{ Test preparation } \\
\hline \multicolumn{2}{|c|}{$\begin{array}{l}\text { Class A, Class B, Property ispartOf and Property } \\
\text { hasPart exist }\end{array}$} & \multicolumn{2}{|c|}{$\begin{array}{l}\text { (ODPPOe 1.1) Declaration of } \neg \mathrm{B} \\
\text { (ODPPOe 1.2) Assertion A' } \sqsubseteq \mathrm{A} \\
\text { (ODPPOe 1.3) Assertion A'(a1) } \\
\text { (ODPPOe 1.4) Assertion } \neg \mathrm{B}(\text { nob1) } \\
\text { (ODPPOe 1.5) Declaration of } \neg \mathrm{A} \\
\text { (ODPPOe 1.6) Assertion B' } \sqsubseteq \mathrm{B} \\
\text { (ODPPOe 1.7) Assertion B'(b1) } \\
\text { (ODPPOe 1.8) Assertion } \neg \mathrm{A}(\text { noa1) }\end{array}$} \\
\hline \multicolumn{4}{|c|}{ Assertions to test the ontology behaviour } \\
\hline \multicolumn{2}{|r|}{ Axiom } & \multicolumn{2}{|r|}{ Result } \\
\hline \multicolumn{2}{|c|}{$\begin{array}{l}\text { (ODPPOe 2.1) Assertion A' } \sqsubseteq \forall i s p a r t O f .\{\text { nob1 }\} \\
\text { (ODPPOe 2.2) Assertion ispartOf(a1, nob1) }\end{array}$} & \multicolumn{2}{|c|}{ Unsatisfiable class } \\
\hline \multicolumn{2}{|c|}{$\begin{array}{l}\text { (ODPPOe 3.1) Assertion A' } \sqsubseteq \forall \mathrm{P} .\{\text { nob1, b1 }\} \\
\text { (ODPPOe 3.2) Assertion ispartOf }(\mathrm{a} 1, \text { nob1) } \\
\text { (ODPPOe 3.3) Assertion ispartOf }(\mathrm{a} 1, \mathrm{~b} 1)\end{array}$} & \multicolumn{2}{|c|}{ Consistent ontology } \\
\hline \multicolumn{2}{|c|}{$\begin{array}{l}\text { (ODPPOe 2.1) Assertion B' } \sqsubseteq \forall \text { hasPart. }\{\text { noa1\} } \\
\text { (ODPPOe 2.2) Assertion hasPart(b1, noa1) }\end{array}$} & \multicolumn{2}{|c|}{ Unsatisfiable class } \\
\hline \multicolumn{2}{|c|}{$\begin{array}{l}\text { (ODPPOe 3.1) Assertion B' } \sqsubseteq \forall P .\{\text { noa1, a1 }\} \\
\text { (ODPPOe 3.2) Assertion hasPart }(\mathrm{b} 1, \text { noa1) } \\
\text { (ODPPOe 3.3) Assertion hasPart }(\mathrm{b} 1, \mathrm{a} 1)\end{array}$} & \multicolumn{2}{|c|}{ Consistent ontology } \\
\hline
\end{tabular}


Table A.25: T25. PartOf ODP between classes A and B with universal restriction

\begin{tabular}{|c|c|c|c|}
\hline Goal: & \multicolumn{3}{|c|}{ T25. PartOf ODP between classes A and B. Universal restriction } \\
\hline Test expression: & \multicolumn{3}{|c|}{ [ClassA] subClassOf [isPartOf] only [ClassB] } \\
\hline Type: & ODP-based test & Related to: & Classes and properties \\
\hline \multicolumn{2}{|c|}{ Test precondition } & \multicolumn{2}{|c|}{ Test preparation } \\
\hline \multicolumn{2}{|c|}{$\begin{array}{l}\text { Class A, Class B, Property ispartOf and Property } \\
\text { hasPart exist }\end{array}$} & \multicolumn{2}{|c|}{$\begin{array}{l}\text { (ODPPOu 1.1) Declaration of } \neg \mathrm{B} \\
\text { (ODPPOu 1.2) Assertion A' } \sqsubseteq \mathrm{A} \\
\text { (ODPPOu 1.3) Assertion } \mathrm{A}^{\prime}(\mathrm{a} 1) \\
\text { (ODPPOu 1.4) Assertion } \neg \mathrm{B}(\text { nob1) } \\
\text { (ODPPOu 1.5) Declaration of } \neg \mathrm{A} \\
\text { (ODPPOu 1.6) Assertion } \mathrm{B}^{\prime} \sqsubseteq \mathrm{B} \\
\text { (ODPPOu 1.7) Assertion } \mathrm{B}^{\prime}(\mathrm{b} 1) \\
\text { (ODPPOu 1.8) Assertion } \neg \mathrm{A}(\text { noa1) }\end{array}$} \\
\hline \multicolumn{4}{|c|}{ Assertions to test the ontology behaviour } \\
\hline \multicolumn{2}{|r|}{ Axiom } & \multicolumn{2}{|r|}{ Result } \\
\hline \multicolumn{2}{|c|}{$\begin{array}{l}\left(\text { ODPPOu 2.1) Assertion } A^{\prime} \sqsubseteq \forall i s p a r t O f .\{\text { nob1\} }\right. \\
\text { (ODPPOu 2.2) Assertion ispartOf(a1, nob1) }\end{array}$} & \multicolumn{2}{|c|}{ Inconsistent ontology } \\
\hline \multicolumn{2}{|c|}{$\begin{array}{l}\text { (ODPPOu 3.1) Assertion } A^{\prime} \sqsubseteq \forall .\{\text { nob1, b2 }\} \\
\text { (ODPPOu 3.2) Assertion ispartOf (a1, nob1) } \\
\text { (ODPPOu 3.3) Assertion ispartOf (a1, b2) }\end{array}$} & \multicolumn{2}{|c|}{ Inconsistent ontology } \\
\hline \multicolumn{2}{|c|}{$\begin{array}{l}\text { (ODPPOu 4.1) Assertion A' } \sqsubseteq \forall .\{\mathrm{b} 1\} \\
\text { (ODPPOu 4.2) Assertion ispartOf(a1, b1) }\end{array}$} & \multicolumn{2}{|c|}{ Consistent ontology } \\
\hline \multicolumn{2}{|c|}{$\begin{array}{l}\text { (ODPPOu 2.1) Assertion A' } \sqsubseteq \forall \text { hasPart. }\{\text { nob1 }\} \\
\text { (ODPPOu 2.2) Assertion hasPart(a1, nob1) }\end{array}$} & \multicolumn{2}{|c|}{ Inconsistent ontology } \\
\hline \multicolumn{2}{|c|}{$\begin{array}{l}\text { (Urs 3.1) Assertion } A^{\prime} \sqsubseteq \forall \cdot\{\text { nob1, b2 }\} \\
\text { (ODPPOu 3.2) Assertion hasPart (a1, nob1) } \\
\text { (ODPPOu 3.3) Assertion hasPart }(\mathrm{a} 1, \mathrm{~b} 2)\end{array}$} & \multicolumn{2}{|c|}{ Inconsistent ontology } \\
\hline \multicolumn{2}{|c|}{$\begin{array}{l}\text { (ODPPOu 4.1) Assertion } A^{\prime} \sqsubseteq \forall .\{\mathrm{b} 1\} \\
(\mathrm{ODPPOu} 4.2) \text { Assertion hasPart }(\mathrm{a} 1, \mathrm{~b} 1)\end{array}$} & \multicolumn{2}{|c|}{ Consistent ontology } \\
\hline
\end{tabular}


Table A.26: T26. Object-Role ODP between classes A and B with existential restriction

\begin{tabular}{|c|c|c|c|}
\hline Goal: & \multicolumn{3}{|c|}{ T26. Object-Role ODP between classes A and B } \\
\hline Test expression: & \multicolumn{3}{|c|}{ [ClassA] subClassOf [isRoleOf] some [ClassB] } \\
\hline Type: & ODP-based test & Related to: & Classes and properties \\
\hline \multicolumn{2}{|c|}{ Test precondition } & \multicolumn{2}{|c|}{ Test preparation } \\
\hline \multicolumn{2}{|c|}{$\begin{array}{l}\text { Class A, Class B, Property isRoleOf and Property } \\
\text { hasRole exist }\end{array}$} & \multicolumn{2}{|c|}{$\begin{array}{l}\text { (ODPPOe 1.1) Declaration of } \neg \mathrm{B} \\
\text { (ODPPOe 1.2) Assertion A' } \sqsubseteq \mathrm{A} \\
\text { (ODPPOe 1.3) Assertion A'(a1) } \\
\text { (ODPPOe 1.4) Assertion } \neg \mathrm{B}(\text { nob1) } \\
\text { (ODPPOe 1.5) Declaration of } \neg \mathrm{A} \\
\text { (ODPPOe 1.6) Assertion } \mathrm{B}^{\prime} \sqsubseteq \mathrm{B} \\
\text { (ODPPOe 1.7) Assertion B'(b1) } \\
\text { (ODPPOe 1.8) Assertion } \neg \mathrm{A}(\text { noa1) }\end{array}$} \\
\hline \multicolumn{4}{|c|}{ Assertions to test the ontology behaviour } \\
\hline & Axiom & & Result \\
\hline $\begin{array}{l}\text { (ODPPOe } 2.1) \text { Ass } \\
(\text { ODPPOe } 2.2) \text { Ass } \\
\end{array}$ & $\begin{array}{l}\text { tion A' } \sqsubseteq \forall i s R o l e O f .\{\text { nob1 } \\
\text { ction isRoleOf(a1, nob1) }\end{array}$ & \multicolumn{2}{|c|}{ Unsatisfiable class } \\
\hline $\begin{array}{l}\text { (ODPPOe 3.1) Ass } \\
\text { (ODPPOe 3.2) Ass } \\
\text { (ODPPOe 3.3) Ass }\end{array}$ & $\begin{array}{l}\text { ation } \mathrm{A}^{\prime} \sqsubseteq \forall i s R o l e O f .\{\text { nob1, b1\} } \\
\text { ation isRoleOf }(\mathrm{a} 1, \mathrm{nob} 1) \\
\text { ation isRoleOf }(\mathrm{a} 1, \mathrm{~b} 1)\end{array}$ & \multicolumn{2}{|c|}{ Consistent ontology } \\
\hline $\begin{array}{l}\text { (ODPPOe } 2.1) \text { Ass } \\
(\text { ODPPOe } 2.2) \text { Ass }\end{array}$ & $\begin{array}{l}\text { ction } \mathrm{B}^{\prime} \sqsubseteq \forall \text { hasRole. }\{\text { noa1\} } \\
\text { ction hasRole }(\mathrm{b} 1, \text { noa } 1)\end{array}$ & \multicolumn{2}{|c|}{ Unsatisfiable class } \\
\hline $\begin{array}{l}\text { (ODPPOe 3.1) Ass } \\
\text { (ODPPOe 3.2) Ass } \\
(\text { ODPPOe 3.3) Ass }\end{array}$ & $\begin{array}{l}\text { ction } \mathrm{B}^{\prime} \sqsubseteq \forall \text { hasRole. }\{\text { noa1, a1\} } \\
\text { rtion hasRole(b1, noa1) } \\
\text { ation hasRole }(\mathrm{b} 1, \mathrm{a} 1)\end{array}$ & \multicolumn{2}{|c|}{ Consistent ontology } \\
\hline
\end{tabular}


Table A.27: T27. Object-Role ODP between classes A and B with universal restriction

\begin{tabular}{|c|c|c|c|}
\hline Goal: & \multicolumn{3}{|c|}{ T27. Object-Role ODP between classes A and B. Universal restriction } \\
\hline Test expression: & \multicolumn{3}{|c|}{ [ClassA] subClassOf [isRoleOf] only [ClassB] } \\
\hline Type: & ODP-based test & Related to: & Classes and properties \\
\hline \multicolumn{2}{|c|}{ Test precondition } & \multicolumn{2}{|c|}{ Test preparation } \\
\hline \multicolumn{2}{|c|}{$\begin{array}{l}\text { Class A, Class B, Property isRoleOf and Property } \\
\text { hasRole exist }\end{array}$} & \multicolumn{2}{|c|}{$\begin{array}{l}\text { (ODPORu 1.1) Declaration of } \neg \mathrm{B} \\
\text { (ODPORu 1.2) Assertion A' } \sqsubseteq \mathrm{A} \\
\text { (ODPORu 1.3) Assertion A'(a1) } \\
\text { (ODPORu 1.4) Assertion } \neg \mathrm{B}(\text { nob1) } \\
\text { (ODPORu 1.5) Declaration of } \neg \mathrm{A} \\
\text { (ODPORu 1.6) Assertion B' } \sqsubseteq \mathrm{B} \\
\text { (ODPORu 1.7) Assertion B'(b1) } \\
\text { (ODPORu 1.8) Assertion } \neg \mathrm{A}(\text { noa1) }\end{array}$} \\
\hline \multicolumn{4}{|c|}{ Assertions to test the ontology behaviour } \\
\hline \multicolumn{2}{|r|}{ Axiom } & \multicolumn{2}{|r|}{ Result } \\
\hline \multicolumn{2}{|c|}{$\begin{array}{l}\text { (ODPORu 2.1) Assertion A' } \sqsubseteq \forall i s R o l e O f .\{\text { nob1\} } \\
\text { (ODPORu 2.2) Assertion isRoleOf(a1, nob1) }\end{array}$} & \multicolumn{2}{|c|}{ Inconsistent ontology } \\
\hline \multicolumn{2}{|c|}{$\begin{array}{l}\text { (ODPORu 3.1) Assertion } A^{\prime} \sqsubseteq \forall i s R o l e O f .\{\text { nob1, b2 }\} \\
\text { (ODPORu 3.2) Assertion isRoleOf }(\mathrm{a} 1, \text { nob1) } \\
\text { (ODPORu 3.3) Assertion isRoleOf }(\mathrm{a} 1, \mathrm{~b} 2)\end{array}$} & \multicolumn{2}{|c|}{ Inconsistent ontology } \\
\hline \multicolumn{2}{|c|}{$\begin{array}{l}\text { (ODPORu 4.1) Assertion A' } \sqsubseteq \forall i s R o l e O f .\{\mathrm{b} 1\} \\
(\mathrm{ODPORu} 4.2) \text { Assertion isRoleOf(a1, b1) }\end{array}$} & \multicolumn{2}{|c|}{ Consistent ontology } \\
\hline \multicolumn{2}{|c|}{$\begin{array}{l}\text { (ODPORu 2.1) Assertion A' } \sqsubseteq \forall \text { hasRole. }\{\text { nob1\} } \\
\text { (ODPORu 2.2) Assertion hasRole(a1, nob1) }\end{array}$} & \multicolumn{2}{|c|}{ Inconsistent ontology } \\
\hline \multicolumn{2}{|c|}{$\begin{array}{l}\text { (ODPORu 3.1) Assertion A’ } \sqsubseteq \forall \text { hasRole. }\{\text { nob1, b2\} } \\
(\text { ODPORu 3.2) Assertion hasRole }(\mathrm{a} 1, \text { nob1) } \\
(\text { ODPORu 3.3) Assertion hasRole }(\mathrm{a} 1, \mathrm{~b} 2)\end{array}$} & \multicolumn{2}{|c|}{ Inconsistent ontology } \\
\hline \multicolumn{2}{|c|}{$\begin{array}{l}\text { (ODPORu 4.1) Assertion A' } \sqsubseteq \forall \text { hasRole. }\{\mathrm{b} 1\} \\
(\mathrm{ODPORu} 4.2) \text { Assertion hasRole }(\mathrm{a} 1, \mathrm{~b} 1)\end{array}$} & \multicolumn{2}{|c|}{ Consistent ontology } \\
\hline
\end{tabular}

Table A.28: T28. Two individuals of classes A and B can be related by property $\mathrm{P}$

\begin{tabular}{|c|c|c|c|}
\hline Goal: & \multicolumn{3}{|c|}{ T28. Two individuals of classes $\mathrm{A}$ and $\mathrm{B}$ can be related by property $\mathrm{P}$} \\
\hline Test expression: & \multicolumn{3}{|c|}{ [ClassA] [PropertyP] [ClassB] } \\
\hline Type: & Simple test & Related to: & Classes and Properties \\
\hline \multicolumn{2}{|c|}{ Test precondition } & \multicolumn{2}{|c|}{ Test preparation } \\
\hline \multicolumn{2}{|c|}{ Class A, Class B and Property P exist } & \multicolumn{2}{|c|}{$\begin{array}{l}\text { (R 1.1) Assertion } \mathrm{A}(\mathrm{a} 1) \\
(\mathrm{R} 1.2) \text { Assertion } \mathrm{B}(\mathrm{b} 1)\end{array}$} \\
\hline \multicolumn{4}{|c|}{ Assertions to test the ontology behaviour } \\
\hline \multicolumn{2}{|r|}{ Axiom } & \multicolumn{2}{|r|}{ Result } \\
\hline \multicolumn{2}{|c|}{ (R 2) Assertion P(b1, a1) } & \multicolumn{2}{|c|}{ Consistent ontology } \\
\hline
\end{tabular}





\section{ANNEX B}

\section{Axioms associated to test expressions}

Table B.1: Axioms associated to test expressions

\begin{tabular}{|c|c|c|}
\hline & Test & Axioms \\
\hline \multicolumn{3}{|r|}{ Simple tests } \\
\hline $\mathrm{T} 1$ & [ClassA] type Class & :A rdf:type owl:Class \\
\hline $\mathrm{T} 2$ & subclassOf & $\begin{array}{l}\text { :A rdf:type owl:Class; } \\
\text { rdfs:subClassOf :B } \\
\text { :B rdf:type owl:Class }\end{array}$ \\
\hline $\mathrm{T} 3$ & $\begin{array}{l}{[\text { ClassA }] \quad \text { disjointWith }} \\
{[\text { ClassB }]}\end{array}$ & $\begin{array}{r}\text { :A rdf:type owl:Class. } \\
\text { :B rdf:type owl:Class; } \\
\text { owl:disjointWith :A }\end{array}$ \\
\hline $\mathrm{T} 4$ & $\begin{array}{l}{[\text { ClassA }] \quad \text { equivalentTo }} \\
{[\text { ClassB }]}\end{array}$ & $\begin{array}{l}\text { :A rdf:type owl:Class. } \\
\text { :B rdf:type owl:Class; } \\
\text { owl:equivalentClass :A }\end{array}$ \\
\hline T5 & [PropertyA] type Property & :P rdf:type rdfs:Property \\
\hline T6 & $\begin{array}{l}\text { [ClassA] subclassOf [Prop- } \\
\text { ertyP] some }[\text { ClassB] }\end{array}$ & $\begin{array}{l}\text { :A rdf:type owl:Class ; } \\
\text { rdfs:subClassOf [ rdf:type owl:Restriction ; } \\
\text { owl:onProperty :P; } \\
\text { owl:someValuesFrom :B } \\
\text { ] . }\end{array}$ \\
\hline $\mathrm{T} 7$ & $\begin{array}{l}\text { [ClassA] subclassOf [Prop- } \\
\text { ertyP] only [ClassB] }\end{array}$ & $\begin{array}{l}\text { :A rdf:type owl:Class ; } \\
\text { rdfs:subClassOf [ rdf:type owl:Restriction ; } \\
\quad \text { owl:onProperty :P; } \\
\text { owl:allValuesFrom :B } \\
\text { ] . }\end{array}$ \\
\hline $\mathrm{T} 8$ & $\begin{array}{l}\text { [PropertyP] characteristic } \\
\text { symmetricProperty }\end{array}$ & :P rdf:type owl:SymmericProperty \\
\hline
\end{tabular}


Table B.1 - Continued from previous page

\begin{tabular}{|c|c|c|}
\hline & Test & Axioms \\
\hline $\mathrm{T} 9$ & $\begin{array}{l}\text { [ClassA] subclassOf [Prop- } \\
\text { ertyP] min [num][ClassB] }\end{array}$ & $\begin{array}{l}\text { A rdf:type owl:Class ; } \\
\text { rdfs:subClassOf [ rdf:type owl:Restriction ; } \\
\text { owl:onProperty :P; } \\
\text { owl:minQualifiedCardinality "[num]"^^xsd:integer; } \\
\text { owl:onClass :B } \\
\text { ] . }\end{array}$ \\
\hline $\mathrm{T} 10$ & $\begin{array}{l}\text { [ClassA] subclassOf [Prop- } \\
\text { ertyP] max [num][ClassB] }\end{array}$ & $\begin{array}{l}\text { A rdf:type owl:Class ; } \\
\text { rdfs:subClassOf [ rdf:type owl:Restriction ; } \\
\text { owl:onProperty :P; } \\
\text { owl:maxQualifiedCardinality "[num]"^^xsd:integer; } \\
\text { owl:onClass :B } \\
\text { ]. }\end{array}$ \\
\hline $\mathrm{T} 11$ & $\begin{array}{lr}{[\text { ClassA }]} & \text { subclassOf } \\
{[\text { PropertyP }]} & \text { exactly } \\
{[\text { num }][\text { ClassB }]} & \end{array}$ & $\begin{array}{l}\text { A rdf:type owl:Class ; } \\
\text { rdfs:subClassOf [ rdf:type owl:Restriction ; } \\
\text { owl:onProperty :P; } \\
\text { owl:qualifiedCardinality "[num]"^^xsd:integer; } \\
\text { owl:onClass :B } \\
\text { ]. }\end{array}$ \\
\hline $\mathrm{T} 12$ & $\begin{array}{l}\text { [ClassA] subclassOf [Prop- } \\
\text { ertyP] only [ClassB] or } \\
{[\text { ClassC }]}\end{array}$ & $\begin{array}{l}\text { A rdf:type owl:Class ; } \\
\text { rdfs:subClassOf [ rdf:type owl:Restriction ; } \\
\text { owl:onProperty :P ; } \\
\text { owl:allValuesFrom [ rdf:type owl:Class ; } \\
\text { owl:unionOf ( }: \mathrm{B}: \mathrm{C}) \\
\text { ] } \\
\text { ] }\end{array}$ \\
\hline $\mathrm{T} 13$ & $\begin{array}{l}{[\text { ClassA] subclassOf [Prop- }} \\
\text { ertyP] only [ClassB] and } \\
{[\text { ClassC }]}\end{array}$ & $\begin{array}{l}\text { A rdf:type owl:Class ; } \\
\text { rdfs:subClassOf [ rdf:type owl:Restriction ; } \\
\text { owl:onProperty :P ; } \\
\text { owl:allValuesFrom [ rdf:type owl:Class ; } \\
\text { owl:intersectionOf( :B :C ) } \\
\text { ] }\end{array}$ \\
\hline T14 & [IndividualI] type [ClassA] & :I rdf:type owl:NamedIndividual, :A . \\
\hline $\mathrm{T} 15$ & $\begin{array}{l}{[\text { PropertyP }] \quad \text { domain }} \\
{[\text { Class }]}\end{array}$ & $\begin{array}{l}\text { P rdf:type rdfs:Property; } \\
\text { rdfs:domain :A. }\end{array}$ \\
\hline $\mathrm{T} 16$ & {$[$ PropertyP] range $[$ Class $\mathrm{A}]$} & $\begin{array}{l}\text { P rdf:type rdfs:Property; } \\
\text { rdfs:range }: A .\end{array}$ \\
\hline \multicolumn{3}{|c|}{ Composed tests } \\
\hline $\mathrm{T} 17$ & $\begin{array}{l}{[\text { ClassA }] \text { subclassOf }} \\
{[\text { ClassB] and }[\text { ClassC }]}\end{array}$ & $\begin{array}{l}\text { A rdf:type owl:Class; } \\
\text { rdfs:subClassOf :B; } \\
\text { rdfs:subClassOf :C } \\
\text { :B rdf:type owl:Class. } \\
\text { :C rdf:type owl:Class. }\end{array}$ \\
\hline
\end{tabular}

Continued on next page 
Table B.1 - Continued from previous page

\begin{tabular}{|c|c|c|}
\hline & Test & Axioms \\
\hline T18 & $\begin{array}{l}{[\text { ClassA }] \text { subclassOf }} \\
{[\text { ClassB }] \text { that }[\text { PropertyP }]} \\
\text { some }[\text { ClassC }]\end{array}$ & $\begin{array}{l}\text { :B rdf:type owl:Class } \\
\text { :A rdf:type owl:Class; } \\
\text { rdfs:subClassOf :B, [ rdf:type owl:Restriction ; } \\
\quad \text { owl:onProperty :P; } \\
\quad \text { owl:someValuesFrom :C } \\
\text { ]. }\end{array}$ \\
\hline T19 & $\begin{array}{l}\text { [ClassA] subclassof [Prop- } \\
\text { ertyP] min [num] [ClassB] } \\
\text { and [ClassB] subclassOf } \\
{[\text { PropertyP] some [ClassC] }}\end{array}$ & $\begin{array}{l}\text { A rdf:type owl:Class ; } \\
\text { rdfs:subClassOf [ rdf:type owl:Restriction ; } \\
\text { owl:onProperty :P; } \\
\text { owl:minQualifiedCardinality "[num]"^^xsd:integer; } \\
\text { owl:onClass :B } \\
\text { ] . } \\
\text { :B rdf:type owl:Class; } \\
\text { rdfs:subClassOf [ rdf:type owl:Restriction ; } \\
\quad \text { owl:onProperty :P; } \\
\text { owl:someValuesFrom :C } \\
\text { ]. }\end{array}$ \\
\hline $\mathrm{T} 20$ & $\begin{array}{l}\text { [ClassA] subclassof [Prop- } \\
\text { ertyP] min [num] [ClassB] } \\
\text { and [ClassB] subclassOf } \\
{[\text { PropertyP] only [ClassC] }}\end{array}$ & $\begin{array}{l}\text { A rdf:type owl:Class ; } \\
\text { rdfs:subClassOf [ rdf:type owl:Restriction ; } \\
\text { owl:onProperty :P; } \\
\text { owl:minQualifiedCardinality "[num]"^^xsd:integer; } \\
\text { owl:onClass :B } \\
\text { ] . } \\
\text { :B rdf:type owl:Class; } \\
\text { rdfs:subClassOf [ rdf:type owl:Restriction ; } \\
\text { owl:onProperty :P; } \\
\text { owl:allValuesFrom :C } \\
\text { ]. }\end{array}$ \\
\hline $\mathrm{T} 21$ & $\begin{array}{l}{[\text { ClassA] subclassOf }} \\
{[\text { ClassB }] \text { and }[\text { ClassC }]} \\
\text { subclassOf [ClassB] that } \\
\text { disjointWith [ClassA] }\end{array}$ & $\begin{array}{l}\text { A rdf:type owl:Class } \\
\text { rdfs:subClassOf :B. } \\
\text { :B rdf:type owl:Class. } \\
\text { :C rdf:type owl:Class; } \\
\text { rdfs:subClassOf :B ; } \\
\text { owl:disjointWith :A. }\end{array}$ \\
\hline
\end{tabular}

Continued on next page 
Table B.1 - Continued from previous page

\begin{tabular}{|c|c|c|}
\hline \multicolumn{2}{|c|}{ Test } & \multirow[b]{2}{*}{$\begin{array}{l}\text { Axioms } \\
\text { :hasParticipant a owl:ObjectProperty ; } \\
\text { rdfs:domain :B; } \\
\text { rdfs:range :A; } \\
\text { owl:inverseOf :isParticipantIn . } \\
\text { :isParticipantIn a owl:ObjectProperty ; } \\
\text { rdfs:range :B; } \\
\text { rdfs:domain :A. } \\
\text { :B rdf:type owl:Class ; } \\
\text { rdfs:subClassOf [ } \\
\quad \text { a owl:Restriction ; } \\
\quad \text { owl:onProperty :hasParticipant ; } \\
\quad \text { owl:someValuesFrom :A } \\
\text { ] . } \\
\text { :A rdf:type owl:Class ; } \\
\text { rdfs:subClassOf [ } \\
\quad \text { a owl:Restriction ; } \\
\quad \text { owl:onProperty :isParticipantIn ; } \\
\text { owl:someValuesFrom :B } \\
\text { ] . }\end{array}$} \\
\hline $\mathrm{T} 22$ & $\begin{array}{l}\text { [ClassA] subclassOf isPar- } \\
\text { ticipantIn some [ClassB] }\end{array}$ & \\
\hline $\mathrm{T} 23$ & $\begin{array}{l}{[\text { ClassA] and [ClassB] }} \\
\text { subclassOf isParticipantIn } \\
\text { some }[\text { ClassC }]\end{array}$ & $\begin{array}{l}\text { owl:SymmetricProperty ; } \\
\text { owl:inverseOf :coparticipatesWith ; } \\
\text { owl:propertyChainAxiom (:isParticipantIn } \\
\text { :hasParticipant ) } \\
\text { :C rdf:type owl:Class ; } \\
\text { rdfs:subClassOf [ } \\
\text { a owl:Restriction ; } \\
\text { owl:onProperty :hasParticipant; } \\
\text { owl:someValuesFrom [ rdf:type owl:Class ; } \\
\quad \text { owl:unionOf ( :A :B) } \\
\text { ] } \\
\text { ]. } \\
\text { :A rdf:type owl:Class ; } \\
\text { rdfs:subClassOf [ } \\
\text { a owl:Restriction ; } \\
\text { owl:onProperty :coparticipatesWith; } \\
\text { owl:someValuesFrom :B } \\
\text { ] . }\end{array}$ \\
\hline
\end{tabular}

Continued on next page 
Table B.1 - Continued from previous page

\begin{tabular}{|c|c|c|}
\hline \multicolumn{2}{|r|}{ Test } & \multirow[b]{2}{*}{$\begin{array}{l}\text { Axioms } \\
\text { :hasPart a owl:ObjectProperty ; } \\
\text { rdfs:domain :B; } \\
\text { rdfs:range :A; } \\
\text { owl:inverseOf :isPartOf . } \\
\text { :isPartOf a owl:ObjectProperty ; } \\
\text { rdfs:range :B; } \\
\text { rdfs:domain :A. } \\
\text { :B rdf:type owl:Class ; } \\
\text { rdfs:subClassOf [ } \\
\quad \text { a owl:Restriction ; } \\
\quad \text { owl:onProperty :hasPart ; } \\
\text { owl:someValuesFrom :A } \\
\text { ] . } \\
\text { :A rdf:type owl:Class ; } \\
\text { rdfs:subClassOf [ } \\
\text { a owl:Restriction ; } \\
\text { owl:onProperty :isPartOf ; } \\
\text { owl:someValuesFrom :B } \\
\text { ] . }\end{array}$} \\
\hline $\mathrm{T} 24$ & $\begin{array}{l}\text { [ClassA] subclassOf is- } \\
\text { PartOf some [ClassB] }\end{array}$ & \\
\hline $\mathrm{T} 25$ & $\begin{array}{l}\text { [ClassA] subclassOf is- } \\
\text { PartOf only [ClassB] }\end{array}$ & $\begin{array}{l}\text { :hasPart a owl:ObjectProperty ; } \\
\text { rdfs:domain :B; } \\
\text { rdfs:range :A; } \\
\text { owl:inverseOf :isPartOf . } \\
\text { :isPartOf a owl:ObjectProperty ; } \\
\text { rdfs:range :B; } \\
\text { rdfs:domain :A. } \\
\text { :B rdf:type owl:Class ; } \\
\text { rdfs:subClassOf [ } \\
\text { a owl:Restriction ; } \\
\text { owl:onProperty :hasPart ; } \\
\text { owl:allValuesFrom :A } \\
\text { ] . } \\
\text { :A rdf:type owl:Class ; } \\
\text { rdfs:subClassOf [ } \\
\text { a owl:Restriction ; } \\
\text { owl:onProperty :isPartOf ; } \\
\text { owl:allValuesFrom :B } \\
\text { ] . }\end{array}$ \\
\hline
\end{tabular}


Table B.1 - Continued from previous page

\begin{tabular}{|c|c|c|}
\hline & Test & Axioms \\
\hline T26 & $\begin{array}{l}{[\mathrm{A}] \quad \text { subclassOf isRoleOf }} \\
\text { some }[\mathrm{B}]\end{array}$ & $\begin{array}{l}\text { :hasRole rdf:type owl:ObjectProperty ; } \\
\text { rdfs:domain :A ; } \\
\text { rdfs:range :B . } \\
\text { :isRoleOf rdf:type owl:ObjectProperty ; } \\
\text { rdfs:range :A ; } \\
\text { rdfs:domain :B ; } \\
\text { owl:inverseOf :hasRole . } \\
\text { :A rdf:type owl:Class ; } \\
\text { rdfs:subClassOf [ } \\
\quad \text { a owl:Restriction ; } \\
\quad \text { owl:onProperty :isRoleOf ; } \\
\quad \text { owl:someValuesFrom :B } \\
\text { ]. }\end{array}$ \\
\hline $\mathrm{T} 27$ & $\begin{array}{l}\text { A] subclassOf isRoleOf } \\
\text { only [B] }\end{array}$ & $\begin{array}{l}\text { :hasRole rdf:type owl:ObjectProperty ; } \\
\text { rdfs:domain :A; } \\
\text { rdfs:range :B. } \\
\text { :isRoleOf rdf:type owl:ObjectProperty ; } \\
\text { rdfs:range :A; } \\
\text { rdfs:domain :B ; } \\
\text { owl:inverseOf :hasRole . } \\
\text { :A rdf:type owl:Class ; } \\
\text { rdfs:subClassOf [ } \\
\quad \text { a owl:Restriction ; } \\
\quad \text { owl:onProperty :isRoleOf ; } \\
\quad \text { owl:allValuesFrom :B } \\
\text { ]. } \\
\text { :B rdf:type owl:Class . }\end{array}$ \\
\hline \multicolumn{3}{|c|}{ Usage tests } \\
\hline T28 & $\begin{array}{l}{[\text { ClassA] }} \\
{[\text { ClassB] }}\end{array}$ & $\begin{array}{l}\text { A rdf:type owl:Class; } \\
\text { rdfs:subClassOf [ rdf:type owl:Restriction ; } \\
\text { owl:onProperty :P; } \\
\text { owl:someValuesFrom :B } \\
\text { ] . }\end{array}$ \\
\hline
\end{tabular}




\section{ANNEX C \\ Usefulness, satisfaction, and ease of use questionnaire about tools for ontology verification}

USE Questionnaire: Usefulness, Satisfaction, and Ease of use.

1. It is useful
$\square$ Strongly Agree
$\square$ Agree
$\square$ Undecided
$\square$ Disagree
$\square$ Strongly disagree

2. It makes the things I want to accomplish easier to get done
$\square$ Strongly Agree
$\square$ Agree
$\square$ Undecided
$\square$ Disagree
$\square$ Strongly disagree

3. It is easy to use

$\square$ Strongly Agree

$\square$ Agree

$\square$ Undecided

$\square$ Disagree

$\square$ Strongly disagree

4. It is user friendly 

$\square$ Strongly Agree
$\square$ Agree
$\square$ Undecided
$\square$ Disagree
$\square$ Strongly disagree

5. Using it is effortless
$\square$ Strongly Agree
$\square$ Agree
$\square$ Undecided
$\square$ Disagree
$\square$ Strongly disagree

6. I can use it without written instructions
$\square$ Strongly Agree
$\square$ Agree
$\square$ Undecided
$\square$ Disagree
$\square$ Strongly disagree

7. I don't notice any inconsistencies as I use it
$\square$ Strongly Agree
$\square$ Agree
$\square$ Undecided
$\square$ Disagree
$\square$ Strongly disagree

8. It is easy to learn
$\square$ Strongly Agree
$\square$ Agree
$\square$ Undecided
$\square$ Disagree
$\square$ Strongly disagree

9. I easily remember how to use it
$\square$ Strongly Agree
$\square$ Agree
$\square$ Undecided
$\square$ Disagree
$\square$ Strongly disagree 
10. I am satisfied with it

$\square$ Strongly Agree

$\square$ Agree

$\square$ Undecided

$\square$ Disagree

$\square$ Strongly disagree

11. It works the way I want it to work
$\square$ Strongly Agree
$\square$ Agree
$\square$ Undecided
$\square$ Disagree
$\square$ Strongly disagree

12. List the most negative aspect(s).

13. List the most positive aspect(s).

14. Optional comments. 